Chapman University

Chapman University Digital Commons

Education (PhD) Dissertations

Dissertations and Theses

Spring 5-2021

\title{
Using a Dual-Factor Model to Understand the Mental Health of Students with School Refusal Behavior
}

\author{
Zachary D. Maupin \\ Chapman University, zmaupin@chapman.edu
}

Follow this and additional works at: https://digitalcommons.chapman.edu/education_dissertations

Part of the Clinical Psychology Commons, Counseling Psychology Commons, and the School Psychology Commons

\section{Recommended Citation}

Maupin, Z. D. (2021). Using a dual-factor model to understand the mental health of students with school refusal behavior [Doctoral dissertation, Chapman University]. Chapman University Digital Commons.

https://doi.org/10.36837/chapman.000266

This Dissertation is brought to you for free and open access by the Dissertations and Theses at Chapman University Digital Commons. It has been accepted for inclusion in Education (PhD) Dissertations by an authorized administrator of Chapman University Digital Commons. For more information, please contact laughtin@chapman.edu. 
Using a Dual-Factor Model to Understand the Mental Health of Students with School Refusal Behavior

\author{
A Dissertation by \\ Zachary D. Maupin \\ Chapman University \\ Orange, CA \\ Donna Ford Attallah College of Educational Studies \\ Submitted in partial fulfillment of the requirements for the degree of \\ Doctor of Philosophy in Education, School Psychology
}

May 2021

Committee in charge:

Amy-Jane Griffiths, Ph.D., Co-Chair

Kelly Kennedy, Ph.D., Co-Chair

Michael Salce, Ed.D.

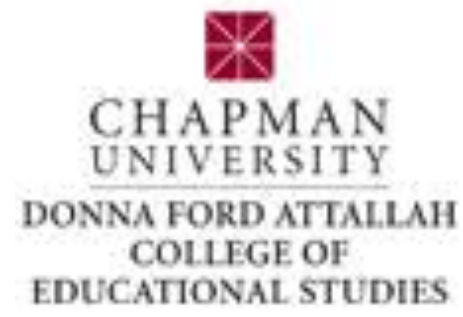


The Dissertation of Zachary D. Maupin is approved.

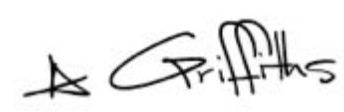

Amy-Jane Griffiths, Ph.D., Co-Chair

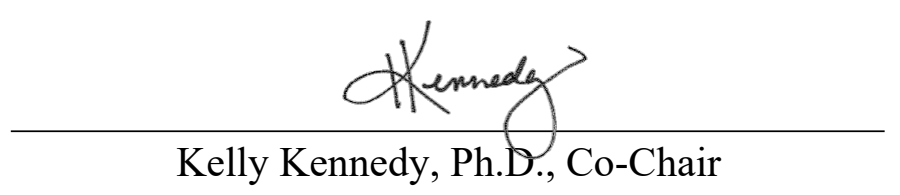

uphe Gla

Michael Salce, Ed.D.

May 2021 
Using a Dual-Factor Model to Understand the Mental Health of Students with School Refusal

Behavior

Copyright (C) 2021

by Zachary D. Maupin 


\section{ACKNOWLEDGEMENTS}

To my wife, Rachel, I could never have done this without you. I will etch your name into my diploma next to mine. I love you. My son, Jack, I promise, no more dissertation drafts for bedtime stories. Mom and Dad, you taught me how to think well and work hard, I am still learning to do both- thank you, I love you. To the rest of my siblings, family, and friends, your patience, support, and understanding will never go unrecognized. Thank you all for filling these years of hard work with wonderful memories and the promise of more to come.

To my Chapman Cohort (2017!) and friends, you are all brilliant. You made this journey fun, memorable, and important. I am excited to watch us all go far and do good things. To all my Chapman Professors and Faculty, your mentorship, guidance, and brilliance have surrounded me with learning, encouragement, and something to strive toward. Dr. Griffiths, I cannot express enough thanks for the opportunities and support you have brought to my life- you have my deepest gratitude. Dr. Kennedy, thank you for tireless Zoom chats and statistical prowess. Dr. Hass, thank you for your wisdom and company. To my Chapman students, you continue to inspire me and fill me with joy and excitement.

To my alma mater, Azusa Pacific University, thank you for starting me on this path and helping me to love learning. Dr. Salce, thank you for challenging me in the classroom and accompanying me on this extended journey. To all the professional organizations and support through NASP, CASP, and ISPA, thank you for sharpening our field and allowing me to participate. To all my professional mentors and colleagues, thank you for your daily differencemaking and ongoing support.

To Dr.'s Furlong, Dowdy, Nylund-Gibson, and all the UCSB faculty from the Covitality Project, thank you for your rich dataset and the encouraging opportunity to partake. 


\begin{abstract}
Using a Dual-Factor Model to Understand the Mental Health of Students with School Refusal Behavior

$$
\text { by Zachary D. Maupin }
$$
\end{abstract}

Students with school refusal behavior (SRB) often present complex cases that include variations of internalizing (anxiety and depression) and externalizing (opposition and defiance) mental health struggles. Historically, incongruent classification methods and terminology have hindered the progress of effectively or consistently assessing SRB. Consequently, practitioners face several obstacles in the process of identifying and understanding these students. Despite guidance from past literature, several questions about how SRB interacts with students' mental health are left unanswered. The present study used data from over 100,000 student responses on the California Healthy Kids Survey-Secondary Core Module. A review of attendance questions from this survey resulted in similar prevalence outcomes as other large surveys. Through additional analysis, select demographic variables (grade, gender, race/ethnicity, socioeconomic status) presented a significant relationship with attendance. These findings were supported by previous research with similar results. Further, Cross-sectional data from the Social-Emotional Health Survey-Secondary and Social-Emotional Distress Scale-Secondary was used to assess students' mental health. Together, these measures reflect a dual-factor approach to mental health that considers both subjective well-being and psychopathology. Responses to these mental health screeners were compared in groups of students based on the amount of school they reported missing or skipping. Findings indicated that students' subjective well-being and psychological distress significantly changed as they missed or skipped school more frequently. Substantially lower reports of subjective well-being and greater reports of psychological distress were found as 
students reported more frequent attendance problems. However, as students began skipping or cutting school once a month or more often in the past 12 months (twice a month, once a week, and more than once a week), there was no longer a statistically significant difference in their mental health. Student responses appeared to plateau as their SRB became more chronic or frequent. These critical findings provided a better understanding of this unique behavior and advanced evidence-based assessment practices for earlier identification of SRB. Considering subjective well-being, in addition to measuring psychopathology, was a crucial component to understanding changes in mental health for students with SRB. These findings have extensive implications for practice and future research. 


\section{TABLE OF CONTENTS}

ACKNOWLEDGEMENTS

$\underline{\text { Page }}$

ABSTRACT V

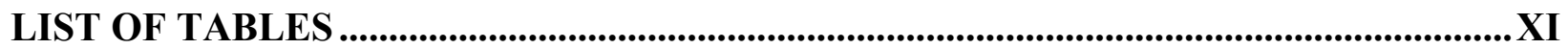

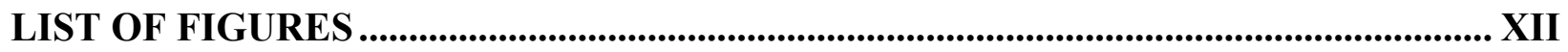

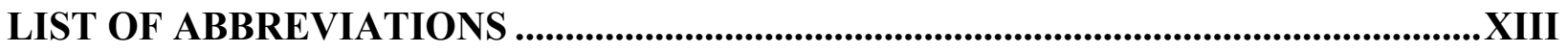

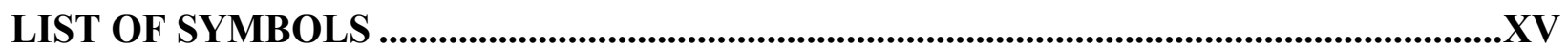

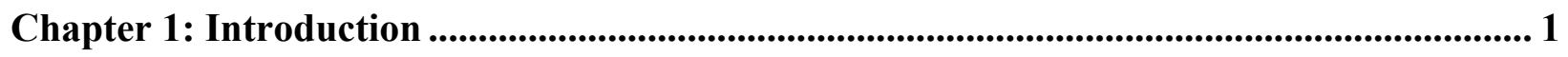

Background Information and Context ............................................................................ 2

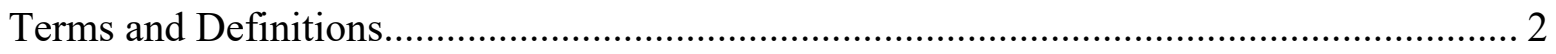

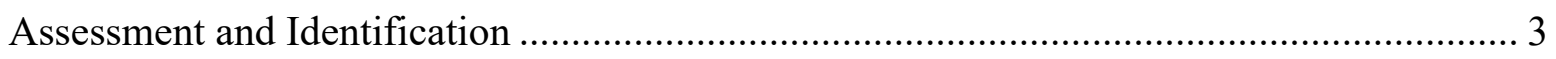

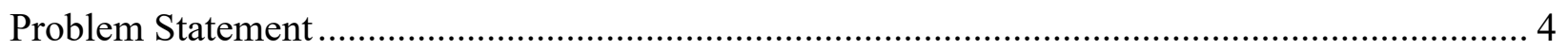

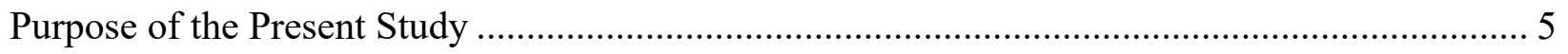

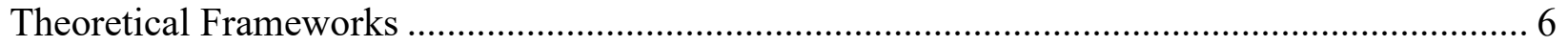

Dual-factor Model of Mental Health ............................................................................. 6

Extant Data Source and Data Agreement ................................................................... 7

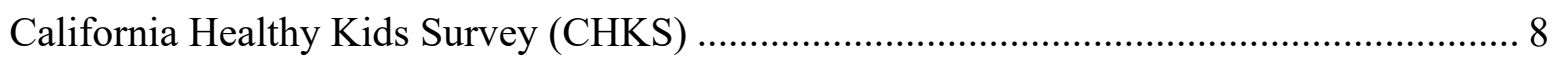

Social-Emotional Health Survey-Secondary ............................................................... 8

Social-Emotional Distress Scale, Secondary (SEDS-S) ........................................... 10

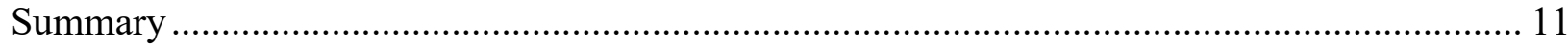

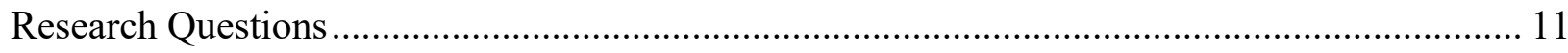

Chapter 2: Literature Review ............................................................................................................... 13

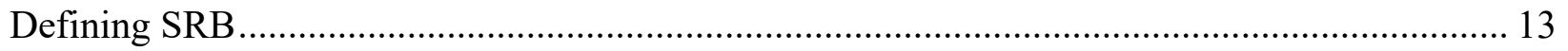

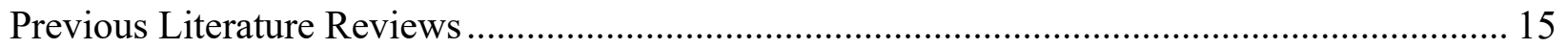

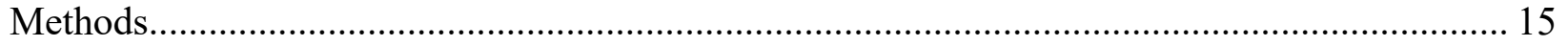

Inclusion \& Exclusion Criteria .................................................................................... 16

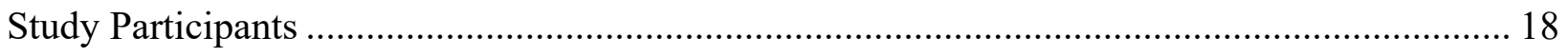

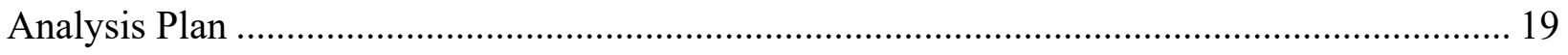




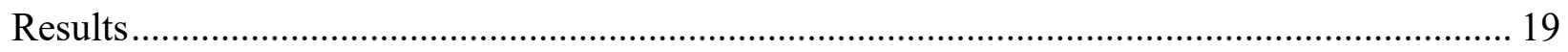

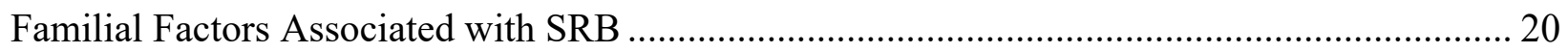

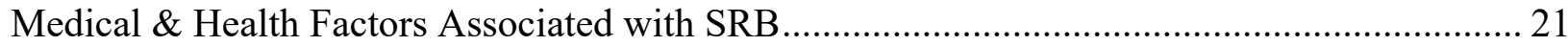

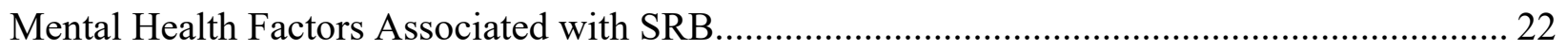

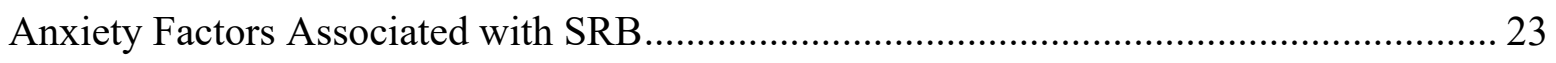

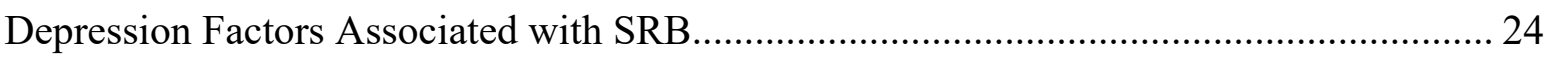

Other Mental Health Factors Associated with SRB …………………………………....... 25

Functional Behavioral Assessment .............................................................................. 26

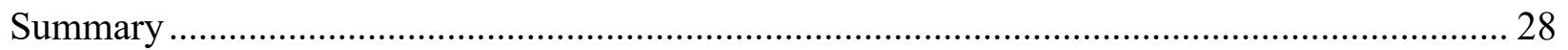

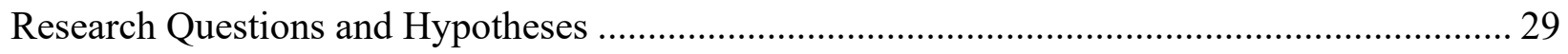

Chapter 3: Research Methodology .......................................................................................................... 35

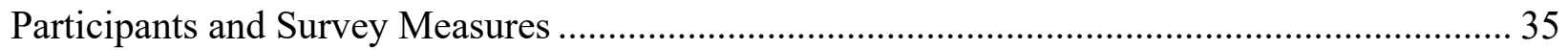

California Healthy Kids Survey (CHKS): Core Module ............................................................. 36

CHKS Design and Psychometrics ................................................................................... 37

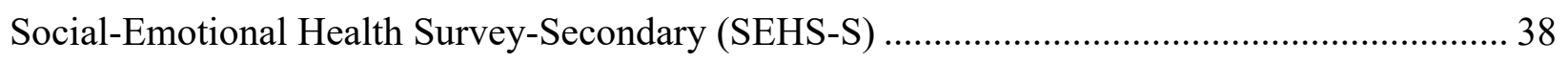

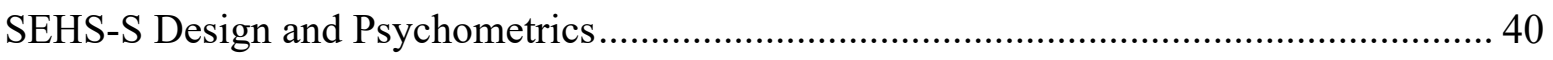

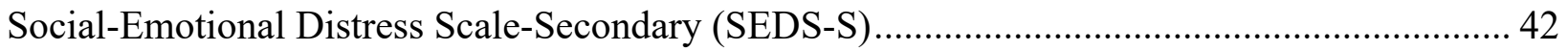

SEDS-S Design and Psychometrics.............................................................................. 43

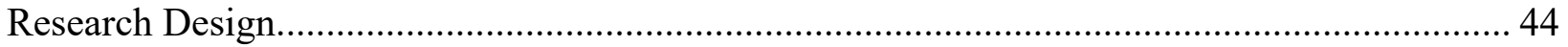

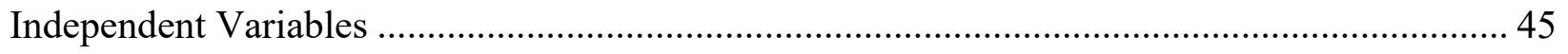

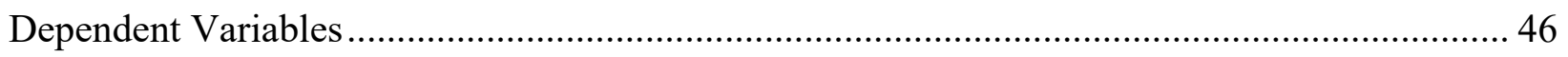

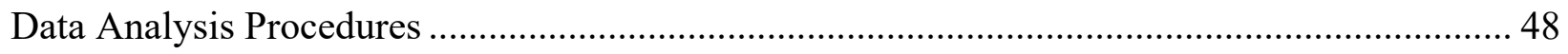

Methodology Descriptions: Analyzing Group Differences ....................................................... 50

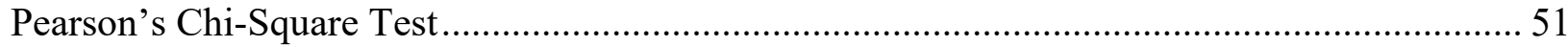

Chi-Square Assumptions …………………………………………………………. 51

Chi-Square Effect Size Calculations................................................................................. 52

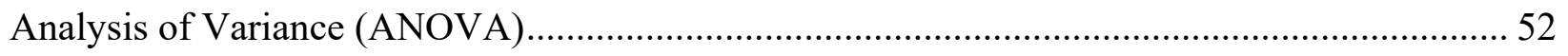

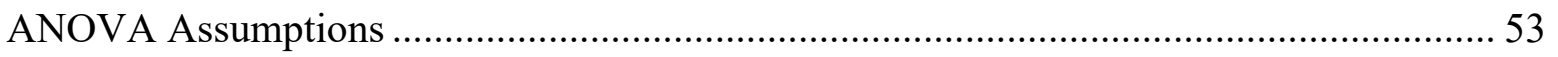

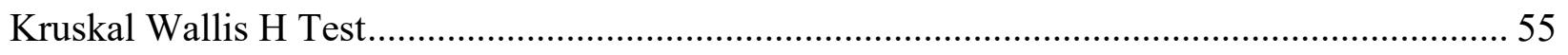

Kruskal Wallis Assumptions.................................................................................... 55

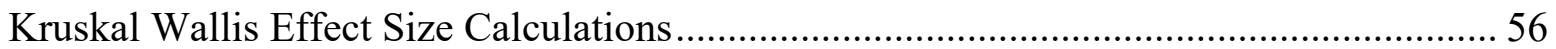

Research Questions, Hypotheses, and Analysis Plans ................................................................. 56 


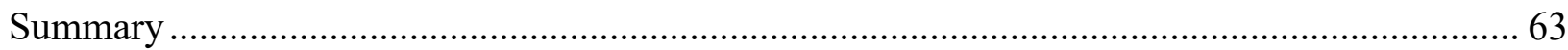

Chapter 4: Results...................................................................................................................................... 65

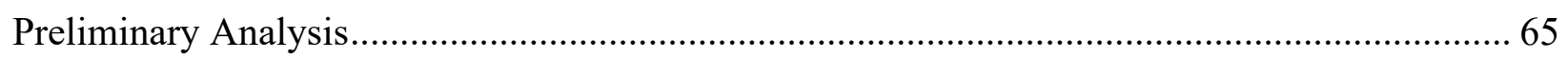

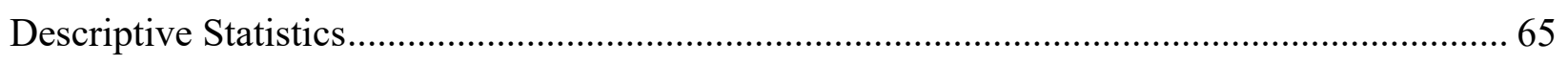

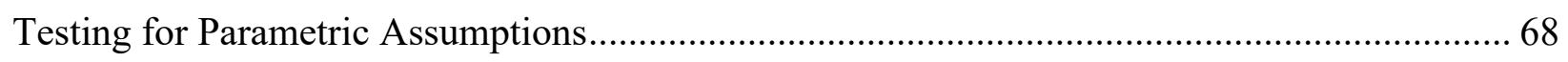

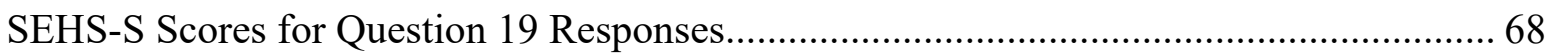

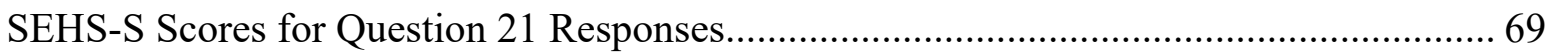

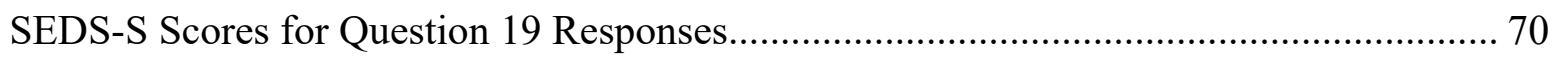

SEDS-S Scores for Question 21 Responses................................................................ 71

Internal Consistency Reliability for the SEHS-S and SEDS-S................................................. 72

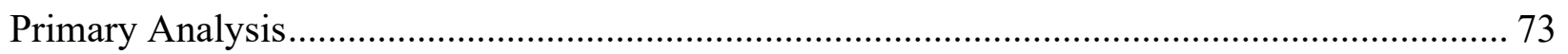

Chapter 5: Discussion ........................................................................................................................................ 94

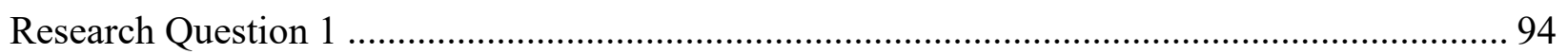

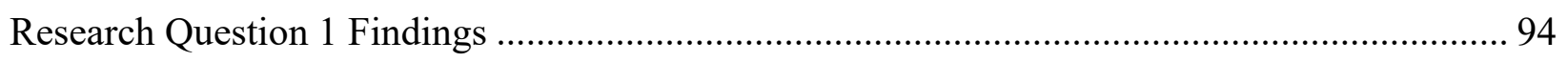

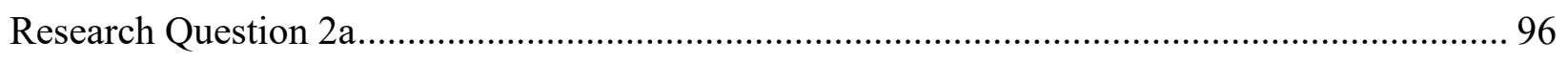

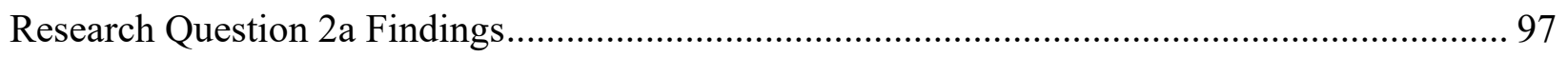

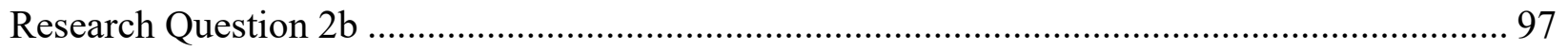

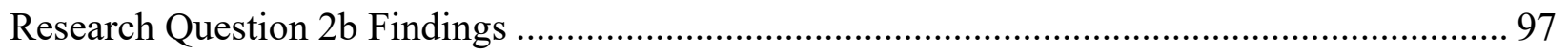

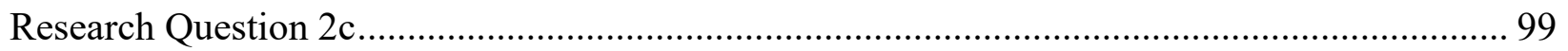

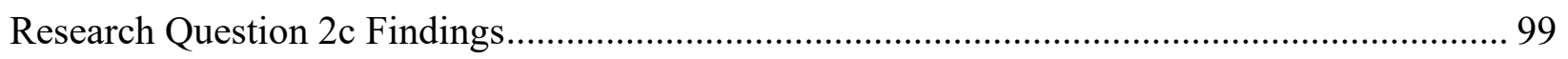

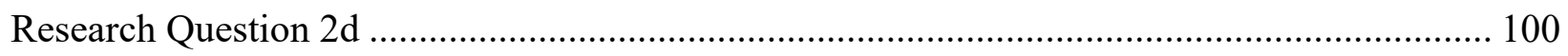

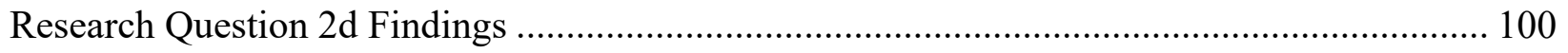

Research Questions 2a-2d Findings Summary ………………………………………....... 101

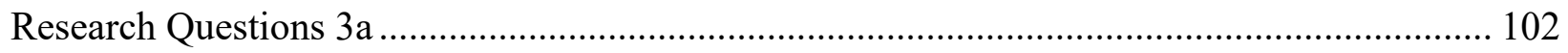

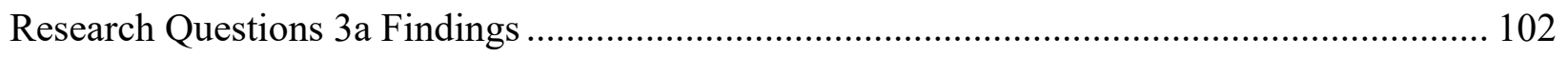

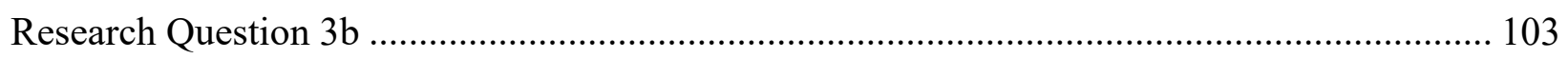

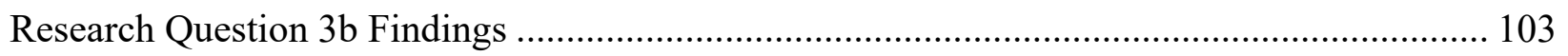

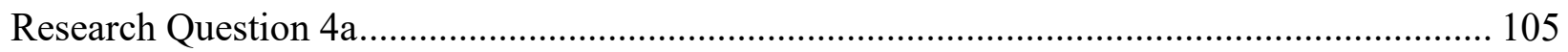

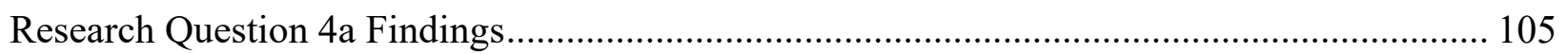

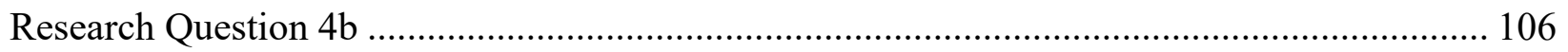

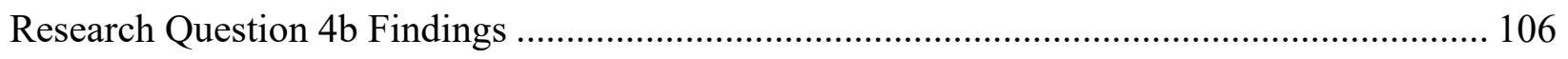


Research Questions 3a-3b and 4a-4b Findings Summary ................................................ 108

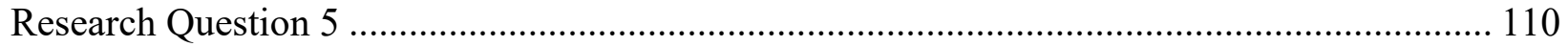

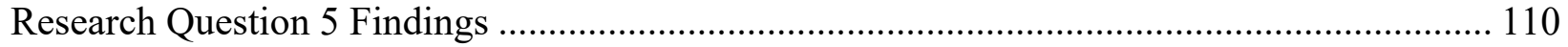

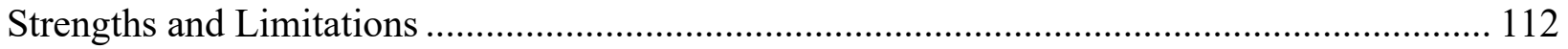

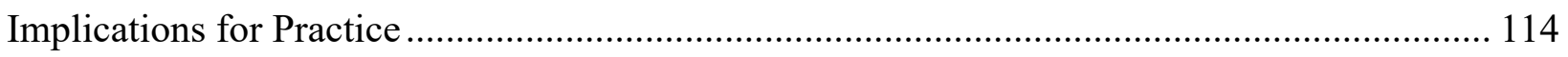

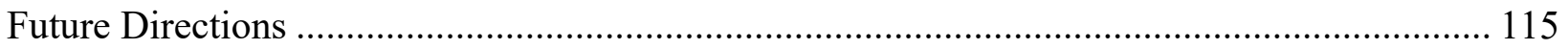

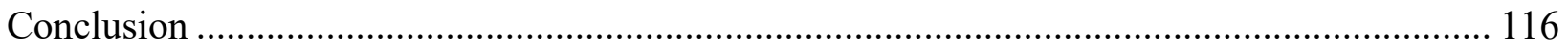

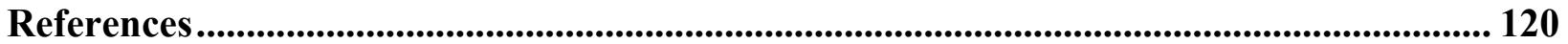

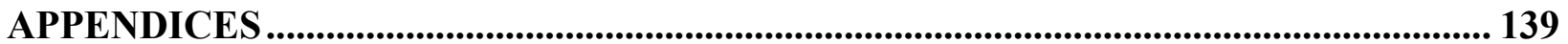




\section{LIST OF TABLES}

$\underline{\text { Page }}$

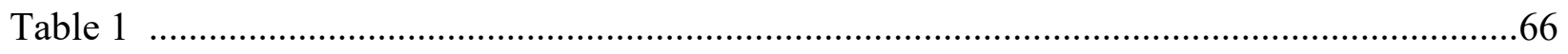

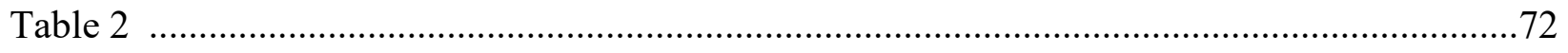

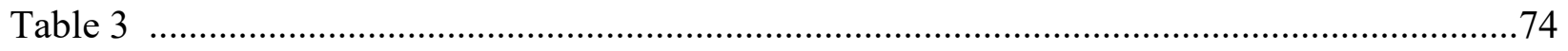

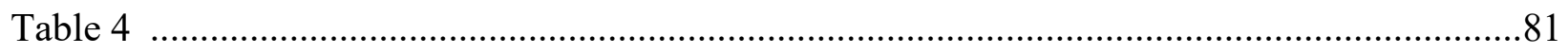

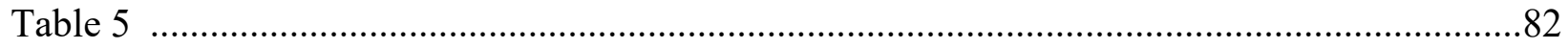

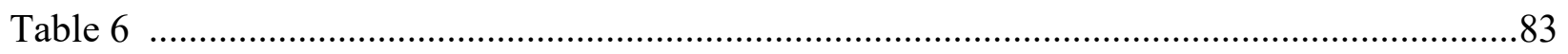

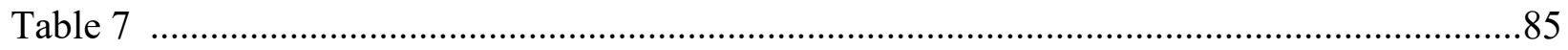

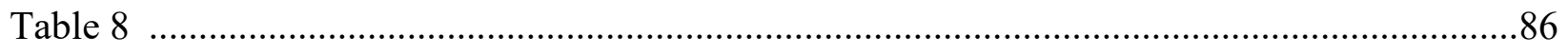

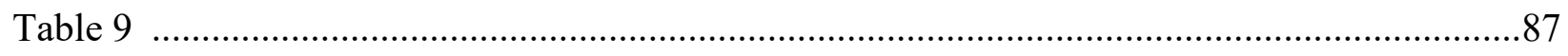

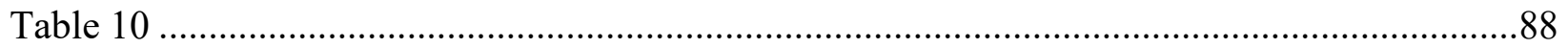

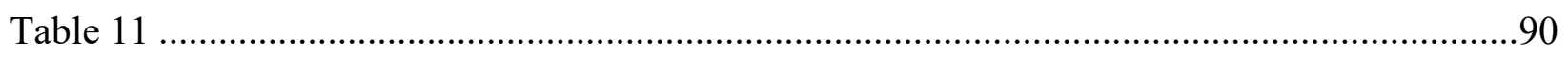

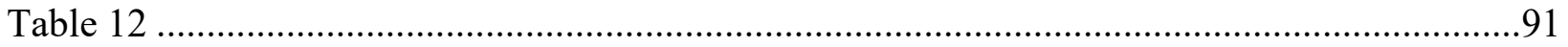

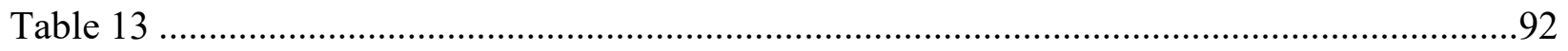




\section{LIST OF FIGURES}

$\underline{\text { Page }}$

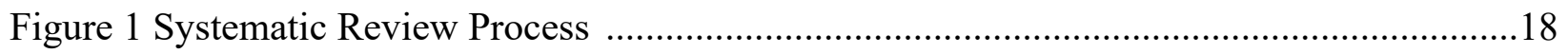

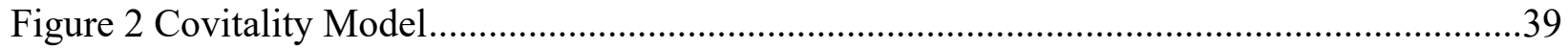

Figure 3 Group Assignment Based on Dual-Factor Model ...............................................47

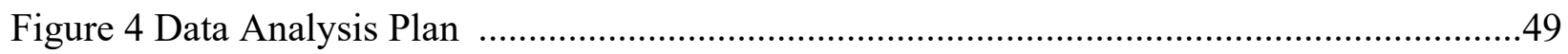




\section{LIST OF ABBREVIATIONS}

\begin{tabular}{|c|c|}
\hline Abbreviations & Meaning \\
\hline AIR & Average Item Response \\
\hline ANOVA & Analysis of Variance \\
\hline APA & American Psychiatric Association \\
\hline BERS & Behavioral Emotional Rating Scale \\
\hline BESS & Behavioral Emotional Screening System \\
\hline BMSLSS & Brief Multidimensional Student Life Satisfaction Scale \\
\hline CFA & Confirmatory Factor Analysis \\
\hline CHKS-SCM & California Healthy Kids Survey-Secondary Core Module \\
\hline CSCRS & Child Self-Control Rating Scale \\
\hline $\mathrm{DF}$ & Degrees of Freedom \\
\hline DSM & Diagnostic and Statistical Manual of Mental Disorders \\
\hline DV & Dependent Variable \\
\hline EFA & Exploratory Factor Analysis \\
\hline ERIC & Education Resources Information Center \\
\hline GAC & Gratitude Adjective Checklist \\
\hline GAD & Generalized Anxiety Disorder \\
\hline ICD & International Classification of Disease \\
\hline IDEA & Individuals with Disabilities Education Act \\
\hline IV & Independent Variable \\
\hline $\mathrm{K}-\mathrm{W}$ & Kruskal-Wallis \\
\hline
\end{tabular}




\begin{tabular}{|c|c|}
\hline Abbreviations & Meaning \\
\hline LCAP & Local Control Accountability Plan \\
\hline LEA & Local Education Agencies \\
\hline LGBT & Lesbian, Gay, Bisexual, Transgender \\
\hline NASP & national association of school psychologists \\
\hline PANAS-C & Positive and Negative Affect Scale for Children \\
\hline PEASS & Experiences at School Scale \\
\hline PHQ & Patient Health Questionnaire \\
\hline PRISMA & Preferred Reporting Items for Systematic Reviews and Meta-Analyses \\
\hline RYDM & Resilience Youth Development Module \\
\hline SAP & school attendance problems \\
\hline SEDS-S & Social-Emotional Distress Scale-Secondary \\
\hline SEHS-S & Social-Emotional Health Survey-Secondary \\
\hline SES & Socioeconomic Status \\
\hline SLSS & Student Life Satisfaction Scale \\
\hline SRAS-R & School Refusal Assessment Scale-Revised \\
\hline SRB & School Refusal Behavior \\
\hline TUPE & Tobacco Use Prevention and Education \\
\hline WHO & World Health Organization \\
\hline YLOT & Youth Life Orientation Test \\
\hline YRBBS & Youth Risk Behavior Surveillance Survey \\
\hline
\end{tabular}




\section{LIST OF SYMBOLS}

\begin{tabular}{cc}
\hline Symbols & Meaning \\
$\alpha$ & Alpha Level \\
$\varphi$ & Phi Effect Size \\
$\Delta($ delta $)$ & Glass' Effect Size \\
$\chi^{2}$ & Chi-Squared \\
$F$ & Ratio of Mean Variance \\
$H$ & Kruskal-Wallis (K-W) $H$ Test \\
$N / n$ & Population/Sample \\
$r$ & Correlation \\
$V$ & Kramer's Effect Size \\
\hline
\end{tabular}




\section{Chapter 1: Introduction}

Public school systems have become an essential component of our society and early childhood development. Compulsory education laws in California require children from 6-18 years of age to attend school (California Department of Education, 2020). Students who regularly do not attend school potentially face several adverse outcomes and a variety of concerns related to learning and academic achievement (Carroll, 2010; Gottfried, 2014), at-risk behaviors (drug use [Chou et al., 2006; Henry \& Huizinga, 2007], teen-pregnancy [Almeida et al., 2006], school drop-out [Christle et al., 2007]), employment and higher education opportunities (Attwood \& Croll, 2006), social-emotional development (Garland, 2001; Hersov, 1990; Malcom et al., 2003), and mental health conditions (Heyne \& Sauter, 2013; Kearney \& Albano, 2004). These difficulties, when left unaddressed, can result in significant problems that persist across a lifetime. Unfortunately, students who refuse to attend school are often unidentified for up to 1-2 years before receiving support (Kearney, 2001).

A variety of complications often accompany cases in which school attendance is a problem. Simultaneous influences may be present in mental health, medical, familial, and contextual factors that can impact attendance. Unfortunately, practitioners may overlook these underlying factors if social maladjustment or other maladaptive behaviors occur (Egger et al., 2003). This dilemma often prevents students with attendance problems from being formally identified or diagnosed with a mental health condition (Kearney, 2007). As a result, students are often unidentified, misunderstood, and face several other barriers to gaining support. This study seeks to understand the mental health changes in students with school attendance problems. 


\section{Background Information and Context}

Concerns about attendance typically present most prominently during significant age or grade transitions (e.g., 5 to 6-, 11 to 13-, and 14 to 15-year-olds) (Kearney et al., 2004; Tolin et al., 2009). As students grow older, attendance problems often increase, and the reasons students refuse school also change. Due to varying criteria and definitions, the prevalence of school attendance problems has included a broad range of 5-28\% of the student population (Kearney, 2001, 2008; King \& Bernstein, 2001). Attendance problems become notably problematic when a child misses (a) $25 \%$ of the total school time in a given two-week period, (b) they demonstrate significant difficulties attending classes for at least two weeks with significant interference to the family or school routines, or (c) they are absent for at least ten days across a fifteen-week period; generally, missing at least $25 \%$ of the school day is considered an absence (Kearney \& Albano, 2018).

\section{Terms and Definitions}

Scholarly discourse has resulted in several attempts to (re)define this phenomenon, including school attendance problems (SAP) (Heyne et al., 2019), extended school nonattendance (Pellegrini, 2007), school refusal behavior (Kearney \& Silverman, 1990 \& 1993), and truancy (Gentle-Genitty et al., 2015; Keppens \& Spruyt, 2017). A general comprehension and understanding of semantics are vital when exploring this topic; however, thorough discussions are reviewed elsewhere (Heyne et al., 2019; Kearney, 2007; Kearney \& Silverman, 1996). Heyne and colleagues (2019) provide a helpful table and timeline of definitions with references in their recent review (p. 9). Unfortunately, interchangeable and incongruent use of terms has resulted in ambiguity, inconsistency, and other substantial obstacles to assessment, identification, treatment, and working knowledge of this topic (Heyne et al., 2019; Kearney, 2003). 
The present study utilized the term school refusal behavior (SRB) as it currently appears most commonly used and well understood throughout the research and practice of assessing attendance problems. A substantial body of research relies on the definition and classification of SRB, amounting to over 30 years of literature. Heyne and company (2019), while proposing a separate classification model and critiques of the term SRB, have recognized the consistency of Kearney and his colleague's definition in literature. Additionally, "SRB" aligns with the National Association of School Psychologists (Wimmer, 2010). NASP recognized "SRB" as a more inclusive term that absorbs students often neglected in the scope of other terms (i.e., truancy and school withdrawal) (Inglés, 2015).

\section{Assessment and Identification}

Students who refuse to attend school present with complex, heterogeneous symptoms that often evade traditional, categorical diagnostic systems (Atkinson et al., 1985; Kearney \& Albano, 2018). While a student with SRB may have mental health struggles (e.g., anxiety, depression, or opposition), their symptoms are often masked, overlooked, or dismissed (Egger et al., 2003; Haight et al., 2011). As a result, these children often do not meet formal criteria or receive a diagnosis, or they are given several (up to five) diagnoses in an attempt to recognize the extent of comorbid mental health struggles (Kearney \& Albano, 2004; Kearney et al., 2005). These complexities have remained detrimental to the identification, classification, and treatment of students with SRB (Elliot, 1999; Elliot \& Place, 2019; Kearney, 2008; King \& Bernstein, 2001; Lauchlan, 2003).

Incongruent terms used in research and practice have also prevented accurate prevalence information from being established (Kearney, 2008; Last \& Francis, 1988). Despite a plethora of terms offered, a unitary nosology does not exist in the American Psychiatric Association's (APA) 
Diagnostic and Statistical Manual of Mental Disorders (DSM-5) or the World Health Organization's (WHO) International Classification of Disease (ICD-10) (APA, 2013; Inglés et al., 2015; WHO, 2004). Further, criteria for special education eligibility in the Individuals with Disabilities Education Act (IDEA) (34 CFR. § 300.34[a]) face similar challenges in its categorical eligibility procedures. A majority of special education disability categories having been unchanged for the past 50 years and present vague descriptions (Triano, 2000) that are unlikely to capture the complex nature of SRB. Literature on SRB significantly lacks guidance studies using school-based data sources or consideration of special education disabilities. As a result, there is confusion on how special education can optimally identify and support this population.

\section{Problem Statement}

In collaboration with the Civil Rights Data Collection (2015-16), the United States Department of Education cited chronic absenteeism as a hidden educational crisis. Based on students missing school in California because they felt unsafe, Baam and colleagues (2017) estimated funding losses of $\$ 276$ million annually. Literature on the topic of assessing, identifying, and understanding SRB has been at a stand-still for the past 20 years. Following their initial article in 1999, Elliot and Place (2019) provided a follow-up literature review on these topics declaring that few advances had been offered to guide practitioners since the turn of the 21st century. As discussed previously, Heyne and colleagues (2019) reviewed chronic incongruencies in the terms and subsequent identification systems used for attendance problems.

Inconsistencies and shortcomings from previous research coupled with heterogeneous mental health symptoms consistent in SRB cases pose a significant problem in research and practice. A further gap is emphasized by the lack of consideration for emerging models of mental 
health- mainly the dual-factor model. This model of mental health considers both subjective well-being and psychopathology to encourage a holistic perspective of mental health. How these combined elements to the dual-factor model present in students with SRB remains a mystery.

\section{Purpose of the Present Study}

Despite a large body of literature on techniques for assessing SRB, there is a clear need for more empirical studies on how school attendance and mental health interact. With recent developments to our comprehension of mental health, assessment procedures should consider additional factors beyond traditional clinical symptoms or deficits (i.e., anxiety, depression, and opposition). In addition to these common psychopathological symptoms (i.e., negative indicators of mental health), the dual-factor model of mental health proposes subjective well-being (i.e., positive indicators of mental health) as critical components to an individual's mental health (Suldo \& Shaffer, 2008). Conceptualizing SRB within a dual-factor lens may reduce the heterogenous roadblocks to successful understanding of students with SRB. As a result, more effective identification and treatment can be developed on account of the whole child.

This study used existing data from a popular school climate measure (California Healthy Kids Survey [CHKS]) as well as measures of subjective well-being (Social-Emotional Health Survey-Secondary [SEHS-S]) and psychological distress (Social-Emotional Distress ScaleSecondary [SEDS-S]). The focus of this study was to analyze groups based on levels of students' attendance to determine what differences exist in their mental health. Select questions from the CHKS were used to create groups based on levels of school absenteeism. Descriptive statistics and initial analysis determined the differences in these groups across grade levels, gender, race/ethnicity, and socioeconomic status. According to the dual-factor model, the SEHS-S and SEDS-S were used to capture a holistic measure of mental health. Scores from these measures 
compared students with varying levels of absenteeism to establish differences in mental health. A copy of select attendance questions from the CHKS, the SEHS-S, and the SEDS-S, are included as Appendices A, B, and C, respectively.

By establishing and analyzing what mental health differences exist across students with different levels of absenteeism, educators can build a more comprehensive understanding of how school attendance and mental health interact. In turn, this supports the development of comprehensive assessment and targeted interventions for students with SRB when considering factors of mental health. Schools, specifically, are urged to use data that is often readily available (i.e., CHKS) to understand and better support students with SRB. Future researchers are encouraged to promote the use of tiered school-based systems and screening procedures to more readily identify and support students with SRB.

\section{Theoretical Frameworks}

Several underlying themes, definitions, models, and tools were relied on for the present study to research the topic of SRB. A brief explanation provided below establishes a basis of knowledge for the reader to understand relevant issues.

\section{Dual-factor Model of Mental Health}

Traditional mental health assessments identify various internalizing (e.g., depression, anxiety) and externalizing (e.g., oppositional or antisocial behavior) symptoms to determine deficits. Essentially, clinicians rely on categorical systems of diagnoses that look for the presence or absence of an illness. The dual-factor model of mental health seeks to expand this approach. This perspective proposed that positive life factors (e.g., life satisfaction, self-acceptance, and social contribution [Diener, 2000]) be considered in addition to psychopathologies (Suldo \& Shaffer, 2008). This conceptualization of complete mental health intends to bolster positive 
assets in addition to identified weaknesses. Both subjective well-being and psychopathology are discernable from one another and necessary to conduct comprehensive evaluations (Greenspoon \& Saklofske, 2001).

The additional spectrum of subjective well-being intersects the spectrum for psychopathologies, creating four discernable quadrants or groups (Suldo \& Shaffer, 2008). Individuals demonstrating high levels of psychopathology with low levels of subjective wellbeing (troubled) are considered most at-risk compared to those with high psychopathology and high subjective well-being (symptomatic but content) or even those with low psychopathology and low subjective well-being (vulnerable). Low psychopathology indicators with reports of high subjective well-being indicate optimal mental health (complete mental health). This model is important for the early identification of mental health struggles. Although there may not be psychopathological concerns, students with low subjective well-being are often unidentified and unlikely to receive support despite their risk of developing mental health struggles (Suldo \& Shafer, 2008).

\section{Extant Data Source and Data Agreement}

The present study used an extensive data set gathered by scholars from the University of California Santa Barbara (Furlong et al., 2020). With support from an Institute of Education Sciences grant initiated in 2017 (Grant \#R305A160157), data collected across the 2017-18 and 2018-19 school years included over 100,000 students in California. This data includes student responses from the California Healthy Kids Survey and concurrent data from the SocialEmotional Health Survey-Secondary (SEHS-S) and Social-Emotional Distress Scale-Secondary (SEDS-S). Each of these measures holds strong validity and reliability and has been designed intentionally for their collective use in measuring complete mental health in the context of 
schools (Furlong et al., 2020; Dowdy et al., 2018). Additional information can be found on the University of California Santa Barbara's Project CoVitality website (Project CoVitality University California Santa Barbara, 2020).

\section{California Healthy Kids Survey (CHKS)}

The California Healthy Kids Survey (CHKS) is a core component of the California School Climate, Health, and Learning Survey (CAL-SCHLS) System; two additional features include school staff and parent surveys. The California Department of Education (CDE), supported by WestEd, Duerr Evaluation Resources, and expert committees, developed the CHKS in response to the federal Safe and Supportive Schools (S3) initiative to enhance positive school climates (WestEd, 2011). The CHKS is now among the oldest and largest statewide surveys of resilience, protective factors, and at-risk behaviors in the nation (Austin et al., 2011).

The CHKS supports priorities aligned with the California Department of Education, the federal government, and Local Control Accountability Plan (LCAP) items for Local Education Agencies (LEAs) intended to foster pupil engagement, parent involvement, academic achievement, Common Core implementation, and basic core services (school facilities) (Zheng et al., 2017). Surveys are specifically available for grades $5,7,9$, and 11 , although other elementary grade levels may appropriately complete the core module. The purpose of this study will focus on the CHKS-Secondary Core Module (CHKS-SCM) items related to attendance, Questions 19 and 21. A copy of Questions 19 and 21 are included as Appendix A.

\section{Social-Emotional Health Survey-Secondary}

The full CHKS core module is required for schools that choose to administer; however, several optional modules explore additional areas of interest. The Social-Emotional Health Survey (SEHS) was developed by researchers at the University of California Santa Barbara to 
measure students' social-emotional strengths based on the Covitality model. This approach is rooted in positive psychology and focuses on strengths related to social-emotional development and well-being (Furlong et al., 2020). Numerous studies have validated this model and the development of 12 sub-scales across the following four secondary measures, belief-in-self (selfawareness, persistence, self-efficacy), belief-in-others (school support, family coherence, peer support), emotional competence (empathy, self-control, behavioral self-control), and engaged living (gratitude, zest, and optimism) (Furlong et al., 2013; Furlong, Dowdy et al., 2014; Furlong, You et al., 2014; You et al., 2014). Together, these constructs result in an individual's Covitality score; this overall measure is greater than the sum of its parts and represents the collective assets that comprise an individual's positive traits (Furlong et al., 2020).

Over time, modifications and expansions have refined the SEHS-S based on factor analysis research (Furlong et al., 2020). The most significant change involved aligning all responses to a four-point scale ("not at all true," "a little true," "pretty much true," and "very much true"); this response format is identical to the scale from the SEDS-S. Questions on the SEHS-S rely on positive indicators for well-being with relatable and appropriate language for adolescent children instead of using pathological (negative) indicators commonly used to describe mental health.

The SEHS-S includes 36 questions to account for the four secondary composites of Covitality and each of their three underlying assets. A standardized scoring process for the SEHS-S follows a rubric outlining the four secondary traits and their three underlying factors. Student responses (1-4) are averaged (divided by three), and each of the subdomains is combined and averaged (divided by nine) to form the composite for each secondary domain. Each secondary domain is added together and averaged (divided by four) to obtain the overall 
Covitality score. Final scores plotted on an Average Item Response (AIR) Profile form indicate average ranges based on 119,756 California students in grades 7-12. A copy of the SEHS-S and a scoring template are included as Appendix B (Furlong et al., 2020).

\section{Social-Emotional Distress Scale, Secondary (SEDS-S)}

The SEDS-S was designed to be administered along with the SEHS-S to encourage a dual-factor approach when measuring positive and negative indicators of mental health and wellness (Dowdy et al., 2018; Furlong et al., 2020). As a broad measure of emotional distress, its use within a school's context can guide further formal or standardized assessment tools. By responding to ten questions about internalizing symptoms in the past month, the SEDS-S measures students' sadness, fear, anxiety, or emotional distress to provide a unitary measure of overall emotional distress and pathology (Dowdy et al., 2018).

Student responses are based on a four-point scale ("not at all true," "a little true," "pretty much true," and "very much true"). Results, averaged (divided by 10) for a total score, have an established average item response score of 2.0 with standard deviations of 1.0 (16-84\% range). Outcomes from the SEDS-S are positively correlated with distress and significantly negatively correlated with positive indicators. A validation study established appropriate internal consistencies (Cronbach's alpha coefficient of .91) when normed across two separate but similar high schools (Dowdy et al., 2018). Additionally, the unitary outcome score for social-emotional distress was supported through confirmatory factor analyses finding an adequate model fit for a single-factor structural model. A copy of the SEDS-S and a scoring template are included as Appendix C (Furlong et al., 2020). 


\section{Summary}

Going to school has become an important developmental component for overall success in our society (Henry \& Huizinga, 2007). Students who struggle to attend school face debilitating mental health struggles as well. These struggles often present as heterogeneous, which can become complex and confusing. Subsequently, classification and identification procedures and informed treatment approaches are significantly lacking. The present study confronted this problem to understand the mental health changes in students at risk for SRB. Subjective wellbeing and psychological distress were both considered in students with varying degrees of SRB to support earlier identification, better assessment practices, and future guidance for more targeted interventions.

\section{Research Questions}

The following research questions guided the analysis of this study and were based on existing literature to address shortcomings concerning our understanding of mental health in students with SRB.

Research Question 1: How do school attendance items from the current dataset compare to prevalence outcomes using other large datasets?

Research Question 2a-2d: Are student self-reports of attendance consistent across demographic items (i.e., [a] grade level, [b] gender and sexual orientation, [c] race/ethnicity, and [d] socioeconomic status)?

Research Question 3a: Are there differences in self-reported subjective well-being measures (i.e., Social-Emotional Health Survey-Secondary [SEHS-S]) across students based on the amount of school they reported missing in the past 30 days (i.e., Question 19)? 
Research Question 3b: Are there differences in self-reported subjective well-being measures (i.e., SEHS-S) across students based on the amount of school they reported skipping or cutting in the past 12 months (i.e., Question 21)?

Research Question 4a: Are there differences in self-reported psychological distress measures (i.e., Social-Emotional Distress Scale-Secondary [SEDS-S]) across students based on the amount of school they reported missing in the past 30 days (i.e., Question 19)?

Research Question 4b: Are there differences in self-reported psychological distress measures (i.e., SEDS-S) across students based on the amount of school they reported skipping or cutting in the past 12 months (i.e., Question 21)?

Research Question 5: Using groups (a) Complete Mental Health, (b) Symptomatic but Content, (c) Vulnerable, and (d) Troubled from a dual-factor model approach, what percentage of students from each group presented with potential SRB? 


\section{Chapter 2: Literature Review}

Several factors can influence school refusal behavior (SRB), including medical factors, mental health and behavioral struggles, and social, familial, and interpersonal dynamics. A critical element to keep in mind is the heterogeneity of SRB- which makes any assumptions about a student's behavior patterns a slight guessing game. Further confusion emerges when secondary features mask a student's primary symptoms or struggles. For example, students may demonstrate oppositional behaviors while struggling with an underlying depression (Bools et al., 1990; Egger et al., 2003). Students with unique medical conditions may refuse school to avoid peer conflict or bullying (Lee et al., 2018). Regardless of why children resist attending school, the results are often dire to their development, learning, families, and society as a whole. Despite research on this topic growing for the past century, this literature remains confusing and inconsistent, urging clarification to understand students with SRB.

\section{Defining SRB}

A history of inconsistent and competing terms and definitions used interchangeably has complicated the topic of attendance problems. This paper will continue to rely on SRB as a more inclusive and flexible term used most frequently in practice and research. It is important to note that multiple factors or behaviors may simultaneously influence a child's absence from school (Kearney, 2002a). Family, community, school, and other contextual aspects of a student's life also significantly influence SRB (Lyon \& Cotler, 2007).

The definition of SRB includes children, 5-17 years old, who refuse to attend school or struggle to remain in class for an entire day (Kearney, 2008). Absenteeism becomes notably problematic when a student (a) misses $25 \%$ of total school time in a given two-week period, (b) demonstrates significant difficulties attending classes for at least two weeks that interfere with 
routines, or (c) is absent for at least ten days during a fifteen-week period; generally speaking, missing $25 \%$ of the school day is considered an absence (Kearney \& Albano, 2018). However, these cutoff points may be arbitrary to a certain degree, and researchers have suggested a lower threshold to promote early identification and prevention (Lyon \& Cotler, 2007). It is reasonable that enough time must surpass for a pattern of absence to be effectively detected.

Recommendations have suggested missing $10 \%$ of school over three months as sufficient to identify cases before SRB becomes less manageable (Lyon \& Cotler, 2007).

Several methods for categorizing absences have included excusable (due to medical illness or injury) or inexcusable (due to environmental, social, or psychiatric factors) absenteeism. Other variations of attendance patterns present in cases of SRB (chronic, acute, periodic, excessive, inconsistent) occur along a spectrum of severity (tardiness, missing a class period, entire days of absence, attending school despite discomfort) (Kearney, 2008). These features often increase the complexity of SRB cases and, subsequently, student concerns may not adhere to traditional diagnostic classification systems. Parent-motivated school withdrawal, for example, can involve parents keeping their child at home for several potential reasons (e.g., safety, child support, economic needs) (Kearney \& Silverman, 1996; Kearney, Lemos, \& Silverman, 2004).

The term SRB has gained distinction for avoiding diagnostic pitfalls and confusion that other classification attempts have faced by emphasizing underlying behavioral functions (Kearney, 2007; Phelp et al., 1992). It is intended to assume previous terms, including truancy, and embrace a universal understanding of this phenomenon that eludes traditional taxonomies and diagnostic systems (Kearney \& Silverman, 1996). The term SRB is not unanimously upheld throughout the literature- primarily due to others refuting the inclusion of truancy-based school 
refusal (Elliot \& Place, 2019; Pellegrini, 2007). However, 30+ years of substantial research supports "SRB" as inclusive and aligned with the National Association of School Psychology (Inglés et al., 2015; Wimmer, 2010). Consensus over terminology is vital for establishing consistency in research and practice and remains a barrier for both (Lyon \& Cotler, 2007).

\section{Previous Literature Reviews}

Several reviews of the literature on assessing SRB provide information identifying and appropriately treating this unique population (Elliot, 1999; Elliot \& Place, 2019; Heyne et al., 2019; Inglés et al., 2015; Kearney, 2008; King \& Bernstein, 2001; Lyon \& Cotler, 2007). These works consistently recognized the value and importance of effective screening and multimodal assessment methods to detect underlying or primary concerns (medical, physical, or psychiatric conditions) and behavioral functions (Elliot, 1999; Elliot \& Place, 2019; Kearney, 2008). Complex heterogeneous features were also recognized as a consistent phenomenon across each of these reviews of SRB.

These reviews provide foundational knowledge and insights; however, they remain unsatisfying in their scope of exploring the assessment of SRB. Their primary focus dwells on the history of incongruent terms and definitions, and recommendations are often limited to anxiety-based screening tools (Elliot, 1999; Elliot \& Place, 2019; Heyne et al., 2019; King \& Bernstein, 2001). The present study includes a systematic review of literature from the past 45 years to discover critical findings on assessing SRB. This information provided context for understanding mental health in students with SRB.

\section{Methods}

The objective of conducting this systematic review was to gain a better understanding of the assessment of SRB. A Boolean search included the terms "assessment" AND "school refusal" 
in four databases including, Education Resources Information Center (ERIC), APA PsycINFO, APA PsycArticles, and MEDLINE. Adding or substituting terms such as "school phobia," "problematic absenteeism," and even "school refusal behavior" only served to decrease the resulting number of articles. In contrast, a term as general as "attendance" added an excessive amount $(9,582)$. The initial search yielded 311 results ranging from 1974 to 2019 . It is important to note that the term school refusal behavior assumed and replaced prior definitions (e.g., school phobia) relatively at this time. The author screened titles and abstracts for each of these articles to remove duplicates and narrow results based on the inclusion criteria presented below. The remaining 119 articles were stored in a Zotero folder to review full article content focusing on methods, results, and discussion sections. Further exclusion criteria specified the use of original or extant data in empirical studies assessing SRB. The author analyzed 40 remaining articles.

\section{Inclusion \& Exclusion Criteria}

An extensive amount of literature was considered to understand what previous research findings suggest for assessing SRB. Recommendations from the Preferred Reporting Items for Systematic Reviews and Meta-Analyses (PRISMA) protocol were employed throughout the literature search and analysis (Moher et al., 2009). Articles were selected for further examination from peer-reviewed academic or medical journals with content focusing on the assessment, identification, and classification of SRB. Parameters for selection included articles that mentioned measurement or assessment related to medical, psychiatric, or mental health factors (e.g., depression, anxiety, attachment, and externalizing or internalizing behavior). Articles that were not empirical by design or did not utilize an original or existing dataset were excluded. A dissertation co-chair provided support with further analyzing article content to ensure a central focus on assessing SRB. 
Articles intended solely for furthering the design and construction of the School Refusal Assessment Scale (across culture, language, and other populations) were excluded to maintain a focus on the overall assessment of SRB. This tool and the research surrounding it include an additional $>81$ articles that warrant a separate and more thorough review. While international sources or studies were maintained, further criteria excluded articles that strictly focused on components of race/ethnicity, culture, or language and its interaction with SRB. Additionally, nine dissertation studies were omitted. Secondary and external reviewers provided further confirmation of article content. From the initial articles selected for consideration, 40 final articles satisfied all inclusion and exclusion guidelines warranting further review for this study. This process was outlined below in Figure 1. 


\section{Figure 1}

Systematic Review Process

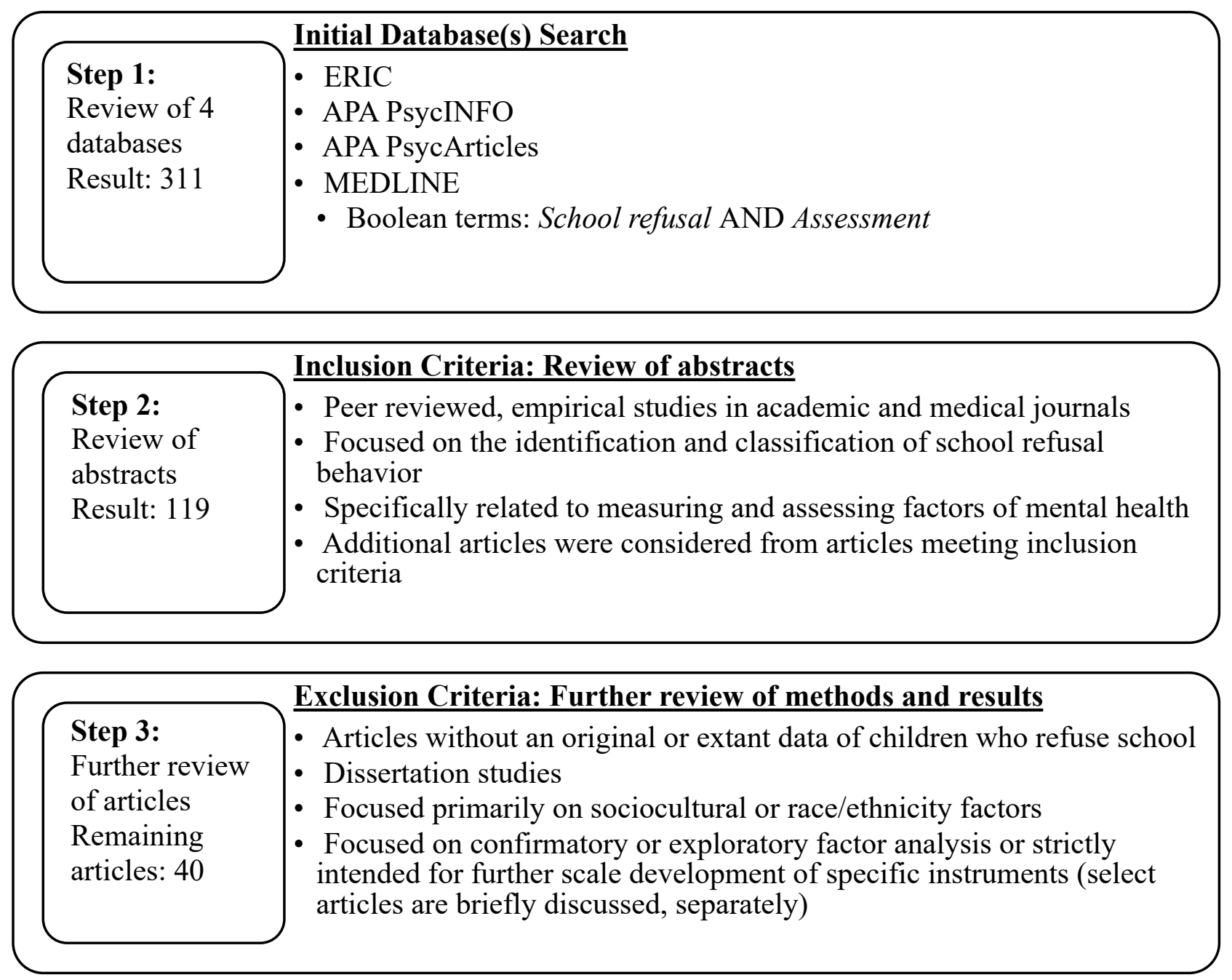

\section{Study Participants}

Across these studies, researchers consistently took into consideration age, gender, race/ethnicity, socioeconomic factors; students ages 5-9 years old were less represented. This growing body of literature spans from studies conducted in the United States, India, Japan, Germany, England, Netherlands, Spain, and other countries, contributing to a better understanding of SRB. However, the settings these studies took place in lacked diversity and primarily took place in clinical settings. Seventy-two percent of the 40 articles selected included 
out-patient or in-patient settings. Further, $90 \%$ of these papers were published in clinical (medical, behavioral, or psychiatric) journal sources. These findings indicate an underrepresentation of educational settings in studies of SRB. Consequently, this potential disparity may influence the portrayal and perception of SRB research.

\section{Analysis Plan}

Following inclusion and exclusion procedures, investigators analyzed content from each article by focusing primarily on the method, results, conclusion, and discussion sections to identify components and critical outcomes for the assessment of SRB. Information about participants (ages, gender, and setting for each study) was compiled into a table with relevant assessment tools and critical implications of the selected work. Themes were identified and further reviewed by secondary and external reviewers. An emphasis remained on identifying critical content relevant to the assessment and implications of SRB to guide researchers and practitioners.

This review established outcomes to analyze regarding the development and understanding of SRB assessments. A list of critical outcomes from this analysis with select details from each study is provided in Appendix D. Results from this review are intended to guide future research and practice by establishing congruent assessment findings to maintain consistency and efficacy. By grounding assessment practices in empirical studies, schools can seek to better support and understand SRB, despite tempestuous changes in terminology and definitions regarding this topic.

\section{Results}

Family dynamics, medical and health factors, mental health (primarily anxiety and depression), and alternative functional assessment methods emerged as common themes in 
assessing SRB. The following section will review critical findings from this literature based on these themes. It became clear that a common link exists between SRB and constructs related to mental health. What became even more evident was the heterogeneous profiles for students with SRB and the urgent need to better understand and address these concerns through early identification, prevention, and interventions. Reviewing these topics was intended to clarify the complex nature of assessing SRB and equip researchers and practitioners with a better understanding of these students. An overview of critical findings from these studies is included as Appendix D.

\section{Familial Factors Associated with SRB}

In earlier literature surrounding SRB, what was then called psychoneurotic-truancy, themes were centered on neuroses related to maladapted family dynamics and parent-child roles. Nearly a quarter of Pritchard and Ward's (1974) sample of students refusing school came from "incomplete families," with $48 \%$ of parents presenting with psychiatric illness. At the time, the prognosis of SRB held tragic outcomes, with only $50 \%$ of children with severe cases returning to school with regular attendance (Valles \& Oddy, 1984). Children who successfully returned to school were highlighted for their family stability as an indicator for more positive outcomes. Research has since found that family dysfunction is more likely influenced by students with disruptive behavioral disorders and substance abuse, while diagnosis and severity of anxiety hold little predictive power for family stability (Bernstein \& Borchardt, 1996; Bernstein et al., 1990).

Considering parent dynamics or family features was vital to understanding, assessing, and effectively supporting SRB. As previously mentioned, parent-motivated school withdrawal is not uncommon in circumstances where the student provides child-care or financial support (Kearney \& Silverman, 1996; Kearney et al., 2004). Additionally, parents of adolescents with 
SRB have reported lower levels of self-efficacy when compared to parents of students with regular attendance (Carless et al., 2015). These dynamics are similar to parents of children who struggle with depression. Several factors limit the availability of literature on family dynamics and SRB, and a profound need for further clarity remains (Lyon \& Cotler, 2007; King \& Bernstein, 2001).

\section{Medical \& Health Factors Associated with SRB}

Attempts to evaluate SRB encounter further complications when medical, health, or developmental factors are present (Arvans \& LeBlanc, 2009; Hochadel et al., 2014; Lee et al., 2018). Whether directly or indirectly, their influence can easily remain overlooked or underrealized. For instance, sleep problems in children with SRB were highly prevalent in young children (8-11) who showed unanimously higher scores across anxiety and depressive disorders (Hochadel et al., 2014). These struggles included insomnias (sleep onset problems, difficulties maintaining sleep), parasomnias (nightmares, night terrors), and daytime sleepiness.

Additionally, students with significant weight gain due to medical complications (e.g., Systemic Lupus Erythematosus) face an increased risk for SRB (Lee et al., 2018).

Children with chronic pain symptoms commonly report more significant cognitive, behavioral, and psychophysiological symptoms of school anxiety when compared to their painfree peers (Gibler et al., 2019). These students were also prone to more anxiety in socially evaluative situations or when faced with peer conflicts. Neurodevelopmental disorders (e.g., autism spectrum disorders) often present with co-occurring medical symptoms (irritable bowel syndrome, sinus infections, stomach aches, allergies, migraines) that have the potential to influence school attendance (Arvans \& LeBlanc, 2009). This expansion of possible contributors or symptoms simultaneously increases the complexity of SRB and the considerations for what 
assessment methods are appropriate and necessary. Yet again, additional research is needed to expand our knowledge of how various health and medical concerns may influence attendance differently with regard to its severity and chronicity and what supports are effective.

\section{Mental Health Factors Associated with SRB}

An undeniable link exists between psychopathologies, or mental health symptoms, and SRB. Unfortunately, students are regularly dismissed or miscategorized due to socially maladaptive behaviors (Egger et al., 2003). Due to the nature of SRB, it frequently becomes viewed solely as an opposition to authority or an intentional breaking of rules. These "truant" behaviors are often considered separate or removed from psychiatric symptoms. However, the presentation of antisocial tendencies or conduct disorder often serves to mask underlying clinical concerns (e.g., depression or anxiety) that frequently contribute to SRB (Bools et al., 1990; Egger et al., 2003).

Kearney and colleagues (2005) analyzed a group of 55 young children aged five to nine years who presented SRB with primary diagnoses of separation anxiety disorders (53.7\%). However, these students' secondary symptoms (disruptive behaviors, opposition, tantrums) often masked core symptoms of anxiety and eluded conventional diagnosis (Kearney et al., 2005). Other primary diagnoses varied and included generalized anxiety disorder (9.3\%), specific phobia (9.3\%), social phobia/avoidant disorder (3.7\%), and enuresis (1.9\%). Additionally, Children often received secondary diagnoses (20.4\% generalized anxiety disorder, $13 \%$ oppositional defiance disorder, $9.3 \%$ separation anxiety disorder). The second leading result of diagnostic assessments for this group of young children resulted in no discernable diagnosis (22.2\%). Kearney and Albano (2004) found similar results with students presenting up to five diagnoses (2.1\%) and several meeting no diagnostic criteria at all (33\%). 
These findings prove troubling when attempting to effectively identify, assess, and treat complex profiles of SRB; a trademark of heterogeneity continues to stifle a clear understanding. Indeed, mental health is connected, but identifying and measuring symptoms becomes severely complicated with environmental and behavioral aspects common in SRB. As a result, diagnostic confusion and misclassification commonly create difficulties when implementing interventions and treatment monitoring.

\section{Anxiety Factors Associated with SRB}

Previously, the term "school phobia" encouraged further consideration for mental health struggles that may influence student absenteeism. Early studies found that younger children who were part of a supportive family and showed lower levels of fear had more positive outcomes (Hansen et al., 1998). Alternatively, older students with more somatic complaints (headaches, abdominal pain, nausea, sweatiness, dizziness, and gastrointestinal symptoms) in conjunction with anxiety symptoms faced less progress in their attempts to return to school.

School anxiety and separation anxiety often present with higher levels of agreement between children and parent ratings; this was likely due to these symptoms being more observable or evident when compared to other areas of anxiety (generalized, social, or specific) (Becker et al., 2016). The severity of somatic symptoms and the extent of a student's absenteeism have a positive correlation (Bernstein et al., 1997; Kearney \& Silverman,1993; McShane et al., 2004; Nayak et al., 2018). Students with anxiety and somatic symptoms were also more likely to struggle with SRB than children with anxiety without somatic complaints (Last, 1991). However, these common somatic complaints are not exclusive to anxiety and are strongly influenced by symptoms of depression (discussed in the next section). 
In addition to somatic complaints, students with anxiety-prone SRB face potentially higher rates of victimization, cyberbullying, and other factors that may exacerbate stress (Delgado et al., 2019). Students with SRB who lacked peer support or faced social rejection continually reported having more significant risk for anxiety, depression, and feelings of inadequacy. In particular, peer rejection was predictive of increasing internalizing symptoms (Craun et al., 2017).

Students with SRB who faced comorbid social phobias and academic difficulties demonstrated less functional improvements over time (McShane et al., 2004). Additionally, a relationship was found between severe social anxiety and the severity and persistence of absenteeism and family dysfunction (Gonzálvez, Kearney et al., 2018). Students who felt unprotected at school faced significant risk factors that may influence the risk of SRB. These crucial dynamics behind school-based interactions and connections should play an important role in developing an understanding for students whose anxiety affects their attendance.

\section{Depression Factors Associated with SRB}

In their study of Japanese students, Honjo and colleagues (2003) established a strong connection between SRB, core depression symptoms, and interpersonal maladaptation. In their prior research, it was contended that children with SRB consistently reported symptoms of depression as secondary to somatic complaints and anxiety symptoms (Honjo et al., 2001). However, two separate studies in India found that a diagnosis of depression was most common (anxiety disorders were second) in students with SRB and that psychiatric illness was much more common in this population when compared to students without SRB (Nayak et al., 2018; Prabhuswamy et al., 2007). Additional studies have supported a strong association between 
depression and anxiety as well as oppositional defiant disorder and conduct disorder (Egger et al., 2003.)

The cultural and environmental aspects of these studies presented critical elements that may influence features of depression. For instance, Nayak and colleagues (2018) found that 33\% of the 45 children in their study reported parental conflicts that included domestic violence or substance abuse by one or both parents. These findings require further research to comprehend how they interact with SRB. A study of Australian families referred for SRB found that depression in a child or parent significantly impacts family dynamics and psychopathologies (Carless et al., 2015).

Making a distinction between anxiety and depression, regardless of SRB, has remained challenging in psychological assessment. Open critiques of the distinction between major depressive disorder and generalized anxiety disorder using the DSM-5 criteria have rendered small reliability statistics at best (Vanheule et al., 2014). Further research is required to perceive how these components to mental health interact distinctly, especially when coupled with the heterogeneity of SRB.

\section{Other Mental Health Factors Associated with SRB}

Studies on students with SRB have expanded to include more holistic views of mental health apart from strictly clinical symptoms. Findings have suggested that children with SRB exhibit thinking patterns more prone to holistic processing as opposed to analytical processing (Rayner \& Riding, 1996). This cognitive style involves viewing information in categorical "wholes" instead of a collection of parts; may contribute to the innate mental obstacles faced by children with SRB. Further studies have found that children with SRB experience more automatic negative thoughts and thinking errors (Maric et al., 2012). Specifically, thoughts of 
personal failure, hostility, and overgeneralization of negative thoughts are potential predictors of SRB. Students with SRB also showed a tendency to contribute select academic failures or outcomes to negative views of inward attributes of themselves instead of external factors (Gonzálvez, Sanmartin et al., 2018). These negative self-appraisals are consistent with traits of depression and anxiety and may contribute to students with SRB having negative inward views or appraisals of themselves.

\section{Functional Behavioral Assessment}

To avoid the shortcomings of traditional assessment approaches and categorical diagnostic criteria, Kearney and Silverman (1988 \& 1990) have spent over 30 years constructing a functional behavioral system for assessing SRB (Kearney, 2002b). This approach considers hypothesis testing for reasons why a student refuses school by using the School Refusal Assessment Scale (SRAS-R) (Kearney \& Silverman, 1999). This assessment method considers externalizing and internalizing factors within (1) negative and (2) positive reinforcement domains that maintain their behavior. Each of these domains has two components, resulting in the following four functions of SRB:

1a. Negative reinforcement: Avoiding specific features or stimuli related to the school setting or its activities that result in distress (anxiety and depression);

1b. Negative reinforcement: Escaping from aversive social scenarios and evaluative situations;

2a. Positive reinforcement: Pursuing attention from significant others (parent or caregiver), often due to separation anxiety that may manifest in somatic complaints and tantrums where the child resists leaving the home setting or presence of a significant other; and 
2b. Positive reinforcement: Seeking preferred or tangible rewards and interactions outside of school (possibly television or video games, social interaction, drug use, shopping) (Kearney \& Albano, 2004; Kearney \& Silverman, 1990).

With proper training, the SRAS provides an effective method for categorizing students based on the underlying, hypothesized function of their SRB (Daleiden et al., 1999). This approach has shown to be effective for determining a prescriptive approach to providing treatment (Kearney \& Silverman, 1999). Extensive research on this tool and its validation exists for further review (Inglés et al., 2015; Kearney \& Silverman, 1990; Kearney \& Albano, 2004; Kearney et al., 2001; Kearney, 2002b). Additionally, researchers have examined translations in German (Walter et al., 2017), Spanish (Gonzálvez et al., 2016), and Turkish (Seçer, 2014) languages (Elliot \& Place, 2019). A substantial asset of this assessment model is its alternative method for categorizing SRB and guiding more prescriptive and effective treatments (Chorpita et al., 1996; Kearney \& Silverman, 1999).

Some variation exists in the presentation of these four functional groups of SRB. For instance, children five to nine years of age do not often avoid school to escape aversive social or evaluative situations. They are most likely to refuse school to pursue attention from a caregiver (Kearney et al., 2005). Overall, cases presenting multiple functions to their SRB sustained the most negative impact and more maladaptive behaviors (Gonzálvez, Kearney et al., 2018). These children often reported more behavioral problems, difficulties coping with victimization, trauma, or stress, poor social functioning, and lower self-concepts (Dube \& Orpinas, 2009; Gonzálvez et al., 2019; Gonzálvez, Kearney et al., 2018).

This functional model offers a needed reprieve from the shortcomings of formal diagnostic categorizations for SRB. However, upholding effective, comprehensive assessment 
methods and collaboration across practitioners, parents, and school staff remain critical (Kearney, 2002a). Additionally, the function of a student's SRB may change or vary over time and require updating and modifying treatment based on ongoing progress monitoring (Kearney, 2007). Underlying medical or psychiatric factors also present as primary or secondary concerns and should be taken into account. Lastly, practitioners should carefully consider family dynamics, developmental status, race/ethnicity, socioeconomic status, comorbid disorders, and the severity of SRB (Kearney, 2007).

\section{Summary}

The topic of school attendance has long been recognized as a growing concern and priority (Kena et al., 2016). Twenty-five percent of students presenting with SRB met the criteria for a DSM-4 disorder compared to only $6.7 \%$ of the regularly attending students (Egger et al., 2003). While expansive knowledge and resources are available for assessing clinical traits in SRB cases (e.g., anxiety or depression), very little is known about how these clinical features interact with SRB. For instance, students with SRB and a psychiatric diagnosis often continue to meet diagnostic criteria even after they gradually return to school (Prabhuswamy et al., 2007). In a longitudinal study, Wood and colleagues (2012) found absenteeism and psychopathology played a reciprocal role in precipitating risk factors for one another. Essentially, the presence of SRB may exacerbate mental health struggles and vice versa.

Although a strong connection between mental health and SRB is suspected, little understanding exists about symptom changes when student absenteeism varies in severity or chronicity. Following a systematic analysis of literature and other reviews, several questions were left unanswered. The present study sought clarity for the prospect of assessing mental health in cases where SRB is present. Additional demographic variables were analyzed to 
determine what essential features practitioners should consider when identifying students at risk for SRB. The present study incorporated an original perspective by using a dual-factor approach to assess students' subjective well-being and psychopathology. This chapter's conclusion provides hypotheses to the research questions presented in Chapter 1.

\section{Research Questions and Hypotheses}

The following research questions were gathered from Chapter 1 and restated with relevant hypothesis statements based on reviewed literature. Suspected outcomes included in these hypotheses were relied on for the proceeding chapters on analysis, results, and discussion. Research Question 1: How do school attendance items from the current dataset compare to prevalence outcomes using other large datasets?

\section{Research Question 1 Hypothesis}

SRB prevalence has varied dramatically and often becomes influenced by inconsistent definitions, terminology, and categorization parameters in each study. Consequently, a broad range from $05-28 \%$ of the population presents with SRB. Other extensive surveys of middle and high school students estimated prevalence to include $11 \%$ of students, increasing to $16 \%$ in high school populations (Henry, 2007; Vaughn et al., 2013). Data from middle and high school students from 2002 to 2014 demonstrated similar results, including $10.8 \%$ in 2002 and $11.1 \%$ of students in 2014 (Maynard et al., 2017). The present study expected self-reported attendance problems to fall within this range provided from prior studies.

Research Questions 2a-2d: Are student self-reports of attendance consistent across demographic items?

Research Question 2a: Are significant differences observed in attendance groups based on grade level? 


\section{Research Question 2a Hypothesis}

Some literature has suggested that peak SRB prevalence most commonly occurred in students ten to thirteen years of age, or during transitions from elementary to middle school or middle school to high school (Kearney \& Albano, 2018; Kearney et al., 2004). However, findings from another large, nationwide data sample $(n=209,393)$ from 2002-2014 consistently found attendance problems increased in adolescents as they grew older (comparing youth ages 12-14 and 15-17) (Maynard et al., 2017). The present study expected high school students to be absent more frequently than middle school students, showing a general increase with age. Research Question 2b: Are significant differences observed in attendance groups based on gender or sexual orientation?

\section{Research Question 2b Hypothesis}

Select studies have supported that absenteeism rates related to truancy are highest in female students (Maynard et al., 2017), while other studies report a significant difference for male students. Findings have remained somewhat inconsistent; however, multiple studies have maintained that gender does not appear to play a substantial role in influencing SRB (Henry, 2007; Kearney et al., 2004; Vaughn et al., 2013). Previous literature regarding LGBT students has found a higher level of risk for SRB (Pampati et al., 2020; Robinson \& Espelage, 2011). The present study expected females to present more frequent absenteeism except for transgender students.

Research Question 2c: Are significant differences observed in attendance groups based on student racelethnicity? 


\section{Research Question 2c Hypothesis}

SRB and related attendance problems present substantial problems for all race/ethnicity groups (Kearney et al., 2004). However, in groups based on patterns of mild, moderate, and chronic attendance problems, research has demonstrated idiosyncrasies when comparing the frequency of absences. For example, Vaughn and colleagues (2013) found Caucasian/White students to represent nearly $80 \%$ of students in a group with mild SRB. While groups of moderate SRB struggles consisted primarily of African American or Hispanic (almost 80\%), students with chronic absences were for the most part evenly distributed across White, African American, and Hispanic students. When comparing attendance for secondary students (ages 1217) in race/ethnicity groups of Non-Hispanic White, African American, and Hispanic, the prevalence of absenteeism was highest for Hispanic students, followed by African American and Non-Hispanic White students (Maynard et al., 2017). The present study expected that absenteeism would be more prevalent in students of color.

Research Question 2d: Are significant differences observed in attendance groups based on socioeconomic status?

\section{Research Question 2d Hypothesis}

SRB presents as a substantial problem across all socioeconomic classes (Kearney et al., 2004). However, research has found that higher income is associated with better school attendance (Gennetian et al., 2018). Further studies have supported that students from lowerincome families and communities are four times as likely to miss $10 \%$ or more of school when compared to higher-income peers (Chang et al., 2008; Gottfried, 2014). When analyzing the interaction between household income and attendance, Maynard and colleagues (2017) found a negative correlation between absence and household income (i.e., higher household income was 
associated with lower rates of absence). The present study expected more frequent absences for students who reported their parents had lower levels of education and students who are eligible for free or reduced-price lunch.

Research Question 3a: Are there differences in self-reported subjective well-being measures (i.e., Social-Emotional Health Survey-Secondary [SEHS-S]) across students based on the amount of school they reported missing in the past 30 days (i.e., Question 19)?

\section{Research Question 3a Hypothesis}

Currently, research has not extensively explored differences in subjective well-being for students with varying levels of absenteeism. Recent research has found that select domains of the covitality model (belief-in-self and belief-in-others) were significant indicators of attendance problems (Wroblewski et al., 2019). Based on its correlation with measures of psychological distress or psychopathologies, the present study expected that subjective well-being would similarly decline in students who reported more significant attendance difficulties.

Research Question 3b: Are there differences in self-reported subjective well-being measures (i.e., SEHS-S) across students based on the amount of school they reported skipping or cutting in the past 12 months (i.e., Question 21)?

\section{Research Question 3b Hypothesis}

As previously mentioned, little-to-no research has explored the subjective well-being of students with SRB. Select factors of subjective well-being, belief-in-others, and belief-in-others, are indicators of SRB (Wroblewski et al., 2019). Based on its strong negative correlation with psychopathologies, the present study expected that subjective well-being would deteriorate with more frequent absenteeism. Considering students' subjective well-being was an essential component in the present study and exploring SRB with a dual-factor perspective. 
Research Question 4a: Are there differences in self-reported psychological distress measures (i.e., Social-Emotional Distress Scale-Secondary [SEDS-S]) across students based on the amount of school they reported missing in the past 30 days (i.e., Question 19)?

\section{Research Question 4a Hypothesis}

Research has consistently identified a strong connection, despite the heterogeneity of SRB, between overall mental health and poor school attendance. Symptoms of anxiety and depression are standard features in several cases of SRB. However, these features may vary depending on a student's age, family dynamics, and experience at school. Despite attempting to differentiate between truancy (externalizing behavioral struggles) and symptoms related to anxiety or depression, research supports internalizing psychopathologies are often present and underlying in students with SRB (Bools et al., 1990; Egger et al., 2003). The complexity of SRB often results in students not meeting diagnostic criteria or multiple comorbid diagnoses being simultaneously met (Kearney \& Albano, 2004; Kearney et al., 2005). The present study expected students with poor school attendance to report higher levels of psychological distress.

Research Question 4b: Are there differences in self-reported psychological distress measures (i.e., SEDS-S) across students based on the amount of school they reported skipping or cutting in the past 12 months (i.e., Question 21)?

\section{Research Question 4b Hypothesis}

Similar to Research Question 4a's hypothesis, Thre present study expected students with more frequent absences to report higher levels of psychological distress. This assumption has consistently remained in previous research. Despite the complexity and variety of mental health struggles presenting in SRB cases, symptoms related to anxiety and depression are often standard 
features. However, these symptoms often become ambiguous as student absenteeism becomes more or less chronic or severe (Kearney \& Albano, 2004; Kearney et al., 2005).

Research Question 5: Using groups (a) Complete Mental Health, (b) Symptomatic but Content,

(c) Vulnerable, and (d) Troubled from a dual-factor model approach, what percentage of students from each group presented with potential SRB?

\section{Research Question 5 Hypothesis}

Research has exposed an undeniable link between mental health and SRB; however, no studies have analyzed changes in students' subjective well-and psychological distress have with their attendance. Suldo and Shaffer (2008) conducted research on the dual-factor model based on an analysis of 349 middle school students. Their findings concluded that $57 \%$ of these students demonstrated complete mental health (average to high subjective well-being and low psychopathology), 13\% were symptomatic but content (average to high subjective well-being and high psychopathology), 13\% were vulnerable (low subjective well-being and low psychopathology), and 17\% were troubled (low subjective well-being and high psychopathology). From these findings, students in the complete mental health group were found to have several positive attributes related to social factors, academic assets, physical health, and better school attendance (Suldo \& Shaffer, 2008). The present study expected that vulnerable and troubled students will report more frequent absences as their subjective well-being decreases and psychological distress increases. 


\section{Chapter 3: Research Methodology}

The present study used screeners for subjective well-being and psychological distress to analyze students' mental health changes as their attendance problems increased. Two questions from the California Healthy Kids Survey-Secondary Core Module (CHKS-SCM) were used to gauge the severity of SRB. Cross-sectional data from self-report measures of studnets' subjective well-being and psychological distress were gathered using the Social-Emotional Health SurveySecondary (SEHS-S) and Social-Emotional Distress Scale-Secondary (SEDS-S), repsectively. These components uphold a dual-factor perspective of mental health. Additionally, demographic variables were analyzed to consider what influence they have on SRB. In the following sections, participants from the present dataset were reviewed, followed by a description of each instrument. After establishing variables and reviewing methodology, an analysis plan was outlined for each Research Question.

\section{Participants and Survey Measures}

The present study relied on a large $(n=107,125)$ extant dataset acquired through a datasharing agreement with the University of California Santa Barba's Covitality Project (Project Covitality, 2021a; Furlong, 2020); a copy of this agreement is included as Appendix E. A grant (\#R305A160157) through the Institute of Education Sciences supported the Covitality Project over the past five years (Project Covitality, 2021b). Included in these data were cross-sectional responses from secondary (grades 7-12) students from 296 public, charter, and alternative secondary schools across 35 California counties. The present data-sharing agreement included responses to select questions from the CHKS-SCM (CalSCHLS, 2021a) and concurrent responses on the Social-Emotional Health Survey-Secondary (SEHS-S) (Furlong, Dowdy et al., 2014; Furlong, You et al., 2014; Furlong et al., 2020) and the Social-Emotional Distress Scale- 
Secondary (SEDS-S) (Dowdy et al., 2018) from the 2017-18 and 2018-19 school years. Inclusion criteria for this project included the following guidance:

1. The English language version of the survey was completed (a separate report examined Spanish language responses).

2. A minimum of 30 (of the 36) SEHS-S items were completed.

3. The CHKS response quality check was passed (the CHKS includes a case rejection criterion that removes students with suspicious responses and inconsistent or outlandish/impossible responses).

4. More than 10 minutes were taken to complete the survey (students who took the survey in under 10 minutes were not included in order to remove low effort responders).

5. Items were answered honestly. The CHKS includes an item as a response quality check (How many questions in this survey did you answer honestly? [responses: all of them, most of them, only some of them, hardly any]). Students responded, hardly any, were not included in the project's cross-sectional sample (Furlong et al., 2020, p 12).

This secondary analysis of data was approved as exempt through the university Institutional Review Board (IRB-21-95) on 12/21/2020.

\section{California Healthy Kids Survey (CHKS): Core Module}

Over the past 20 years, the CHKS has become a comprehensive measure of school climate that includes multiple modules to survey students' risk, resilience, and resources (Mahecha \& Hanson, 2020). The Core Module has remained a mandated component to California state schools receiving Tobacco Use Prevention and Education (TUPE) grants. It also supports compliance with the No Child Left Behind Act and is intended to guide Local Control 
and Accountability Plan (LCAP) efforts. Traditionally, participating schools administer the survey every other year; however, several schools consider annual administration. In the 2017/18 and 2018/19 school years, the CHKS-SCM was administered to 1,179,951 students in 2,953 schools across 717 districts. This represented nearly $70 \%$ of the districts and $52 \%$ of schools in California. Almost $30 \%$ of these participating districts completed the CHKS annually.

\section{CHKS Design and Psychometrics}

Core Module surveys are now available for elementary (41-6), middle (7-8), and high school $\left(9-12^{2}\right)$ grade levels, with administration guidance available online (CalSCHLS, 2021b). Testing duration for the CHKS-SCM lasts approximately one class period; $95 \%$ of middle school students can complete the online Core Module in about 30 minutes, while high school students take about 24 minutes. Administrating additional modules requires more time but can be customized to meet the needs and specifications of any district. Typically, this self-report survey was completed anonymously following passive consent from parents and student assent.

Over the years, the CHKS has undergone extensive psychometric testing and development (Hanson, 2011; Hanson, 2012; Hanson \& Kim, 2007; Hanson \& Voight, 2014). Mahecha and Hanson (2020) provided a recent report that reviewed data from the 2017/18 school year. Findings from this report continued to confirm the established nine distinct dimensions of school climate and student well-being measured in the Secondary Core Module (Hanson, 2011; Hanson \& Voight, 2014). The discriminant validity of these factors was appropriately small, with one exception (Violence Victimization and Harassment and Bullying have a higher correlation of 0.86 ). Through a confirmatory factor analysis model, the average

\footnotetext{
${ }^{1}$ Traditionally, the Elementary Core Module is intended for grades $5 \& 6$, however, students in grade 4 can be accommodated.

${ }^{2}$ The Secondary Core Module also includes nontraditional grades or settings (e.g., continuation school).
} 
loading across all constructs was 0.81 . These results indicated that each item was appropriately correlated to its underlying factor (Mahecha \& Hanson, 2020).

Further analysis of the Secondary Core Module from 556,961 students across 2,187 schools revealed no substantial item bias across demographic questions related to grade levels $(7$, 9, 11, and non-traditional), gender (male, female), race/ethnicity (African American, American Indian, Asian, Latinx, Pacific Islander, White, and Multiethnic), and English language proficiency (English only, English proficient, and Not proficient) (Mahecha \& Hanson, 2020). Some meaningful differences exist between students in each demographic group. For example, items related to "harassment" have a different meaning for females than males. Additionally, nearly all non-white racial/ethnic groups reported higher levels of harassment related to race/ethnicity/national origin and immigrant status (even when controlling for overall levels of harassment and bullying victimization). Estimates for internal consistency reliability for the total sample, based on demographic items, were above the recommended threshold ( $>0.70)$; one exception involved delinquency in female students (Mahecha \& Hanson, 2020). The present study used only select questions (19 and 21) regarding student attendance.

\section{Social-Emotional Health Survey-Secondary (SEHS-S)}

The Social-Emotional Health Survey (SEHS), originally named the Positive Experiences at School Scale (PEASS), was developed out of a positive psychology focus to measure socialemotional strengths in what is known as covitality (Furlong et al., 2013; Furlong, Dowdy et al., 2014; Furlong, You et al., 2014). This construct consists of four domains, each with three subsequent subdomains (Engaged Living [Optimism, Zest, Gratitude], Emotional Competence [Emotional Regulation, Self-Control, Empathy], Belief-in-Others [Family Support, Peer Support, School Support], and Belief-in-Self [Self-Awareness, Self-Efficacy, Persistence]). Each of these 
are essential components to an individual's subjective; however, they are collectively viewed as building blocks for an even more important overall combination of strengths (Furlong, Dowdy et al., 2014). In other words, the resulting covitality score from the SEHS has synergistic properties that are greater than each of its co-occurring parts and provide protective assets against emotional and behavioral problems (Lenzi, Furlong et al., 2015; Lenzi, Sharkey et al., 2015). Figure 2 provides an overview of this dynamic model and each of its subcomponents.

\section{Figure 2}

\section{Covitality Model}

\begin{tabular}{|c|c|c|c|c|c|c|}
\hline & & Subdomains & & & & Domains \\
\hline Emotional Regulation & + & Self-Control & + & Empathy & $=$ & $\begin{array}{c}\text { Emotional Competence } \\
+\end{array}$ \\
\hline Optimism & + & Zest & + & Gratitude & $=$ & $\begin{array}{c}\text { Engaged Living } \\
+\end{array}$ \\
\hline Self-Awareness & + & Self-Efficacy & + & Persistence & $=$ & $\begin{array}{c}\text { Belief-in-Self } \\
+\end{array}$ \\
\hline Family Support & + & Peer Support & + & School Support & $=$ & $\begin{array}{l}\text { Belief-in-Others } \\
\qquad= \\
\text { Total Covitality }\end{array}$ \\
\hline
\end{tabular}

The Social-Emotional Health Survey-Secondary (SEHS-S) is now an optional module within the CHKS that measures subjective well-being in children grades 7-12. Initially, the scale included 51 items to measure the covitality and its twelve constructs mentioned above (Furlong, You et al., 2014). These items were intentionally reduced to 36 questions to encourage a brief screener while upholding strong psychometric qualities. Similarly, all questions have been aligned to include a 4-point response scale $(1=$ not at all true, $2=$ a little true, $3=$ pretty much true, 4 = very much true) (Furlong et al., 2020). You and colleagues (2014) had previously 
maintained the gratitude and zest subscales with a 5-point response scale. The resulting tool provides a strong measure of subjective well-being and supports a duo continuum approach to screening mental health struggles in schools (Furlong, You et al., 2014). A copy of the SEHS-S is included as Appendix B

\section{SEHS-S Design and Psychometrics}

The SEHS was developed from modifications to one of the CHKS optional modules, the Resilience Youth Development Module (RYDM). Other measures of positive psychological traits related to well-being were also used as a theoretical foundation (Gratitude Adjective Checklist [GAC], Youth Life Orientation Test [YLOT], Behavioral Emotional Rating Scale [BERS], and Child Self-Control Rating Scale [CSCRS]) (Furlong, You et al., 2014). Convergent validity was investigated using an item related to academic achievement from the CHKS-SCM and two items related to perceived school safety on the California School Climate Index. Discriminant validity procedures relied on three items from the CHKS-SCM related to at-risk behavior (tobacco use, marijuana use, binge drinking) as well as a question related to selfreported depression from the Youth Risk Behavior Surveillance Survey (YRBSS). Concurrent validity was investigated with comparisons to the Student Life Satisfaction Scale (SLSS) and Positive and Negative Affect Scale for Children (PANAS-C)- these measure overall life satisfaction and emotional experiences, respectively.

In their initial validation study, Furlong and colleagues (2014) selected surveys from 3,189 students in grades 8, 10, and 12 from 12 schools (seven junior high schools, four comprehensive high schools, and one continuation high school) in central California. In the 2011-12 school year, students were administered the SEHS-S and the SLSS, PANAS-C, and the CHKS-SCM. Before and after reducing the scale to 35 items, a series of confirmatory factor 
analysis procedures conducted across random samples supported good factor loadings for the covitality construct and subsequent factors. Multigroup invariance testing conducted at three levels (configural, metric, and scalar) supported sufficient invariance for the covitality measure across gender; this implied that a similar construct was measured across gender. By using a structural path model analysis, the four domains (Engaged Living, Emotional Competence, Belief-in-Others, Belief-in-Self) and overall covitality measures proved to be a good fit model with strong positive relations.

Discriminant validity analysis revealed significant mean differences between groups based on the SEHS-S (very low, low, high, and very high) and the measures of at-risk behavior on the CHKS (tobacco use, marijuana use, binge drinking). Similar results were found for the depressive symptoms question from the YRBSS; students with the highest SEHS-S scores were least likely to report symptoms of depression. Analysis of convergent validity also indicated that these groups of covitality scores held significant differences across the self-reported academic achievement question on the CHKS (students in the highest-scoring covitality group reported the highest grades). Similar results found that these students (highest-scoring covitality group) shared higher reports of perceived safety at school based on items in the CHKS. Overall, this original sample maintained strong internal consistency reliability $(\alpha=0.92)$ on the SEHS-S, and covitality scores demonstrated a strong correlation with other measures of well-being $(r=.89)$ (Furlong, You et al., 2014).

In the following school year, You and colleagues (2014) supported these results with a separate sample including 2,240 students from grades 9-12 $(\alpha=0.91)$. Covitality and its underlying factors were, again, supported as a good model fit showing strong factor loading and sufficient invariance across gender-by-age groups (You et al., 2014). Further support for the 
concurrent validity of the SEHS-S used the Behavioral Emotional Screening System (BESS) and found a negative correlation $(r=-.63)$. These findings condoned the SEHS-S as a robust measure of positive psychological traits instead of negative indicators identified on the BESS. The positive associations of covitality and students' academic achievement, measured by their grade point averages, provided additional support of its validity (You et al., 2014).

Due to original study samples consisting of approximately $70 \%$ Latinx or Hispanic students, primarily in the central region of California, limitations were acknowledged for its use across diverse populations (Furlong, You et al., 2014; You et al., 2014). To build more substantial validity and expand consideration for using the SEHS-S, You and colleagues (2015) Surveyed 14,171 students in grades 9-12 across 17 high schools in suburban and urban school districts from San Diego to San Francisco, California. Confirmatory factor analyses continued to support strong factor loadings on each corresponding factor and the overall covitality measure. Further invariance was also established across gender using different sociocultural groups.

Additional studies have supported the ongoing development of the SEHS-S and its use across more diverse groups (Furlong et al., 2020). Strong internal consistency continues to be found for overall covitality scores across Japanese ( $\alpha=0.93$ [Ito et al., 2015]) and South Korean $(\alpha=0.94$ [Lee et al., 2016]) high school students. Additionally, Turkish, Korean, and Chinese youth demonstrated positive associations for high covitality scores and prosocial and strengthsbased measures and negative associations with negative psychological indicators (e.g., depression, anxiety, stress) (Lee et al., 2016; Telef \& Furlong, 2017; Wang et al., 2018).

\section{Social-Emotional Distress Scale-Secondary (SEDS-S)}

The Social-Emotional Distress Scale-Secondary (SEDS-S) was designed to be coadministered alongside the SEHS-S to support the dual-factor model of mental health (Dowdy et 
al., 2018). Its purpose is to assess personal emotional distress within the context of a school. Intended as a screening tool, the SEDS-S is recommended for use prior to more traditional tools when assessing for clinical diagnosis. The SEDS-S was intentionally designed to be short in length and does not differentiate between depression and anxiety but produces a unidimensional measure of internalizing psychological experiences related to these constructs. The initial 10item scale (Dowdy et al., 2018) included 5-point responses $(1=$ not true of me, $2=$ a little true of me, 3 = pretty much true of me, $4=$ true of me, 5 = very true of me), however, a 4-point scale was used in gathering current data $(1=$ not at all true, $2=$ a little true, $3=$ pretty much true, $4=$ very much true) (Furlong et al., 2020). A copy of the SEDS-S is included as Appendix C.

\section{SEDS-S Design and Psychometrics}

In their initial validation study, Dowdy and colleagues (2018) used surveys from 3,780 students in grades 9-12 from two high schools in separate districts in central California. In the 2015-16 school year, they administered the SEDS-S and the SEHS-S to students from both schools. Participants from one of the schools also completed the Brief Multidimensional Student Life Satisfaction Scale (BMSLSS), Patient Health Questionnaire (PHQ-9) Depression Scale, and Generalized Anxiety Disorder-7 Scale (GAD-7) for use in external validity analysis.

Randomized split samples from one school underwent confirmatory and exploratory factor analyses (CFA and EFA) followed by cross-validation with the second school to examine and support an underlying single-factor structure in the SEDS-S (Dowdy et al., 2018). Factor loading remained strong for each of the ten items across both schools and each split-sample analysis. Continually strong internal consistency estimates $(\alpha=.91)$ were found across each school with equally strong latent-level reliability $(\Omega=.91)$. Convergent and discriminant validity were analyzed through structural modeling and path analyses which found significantly positive 
relations between the SEDS-S and the GAD-7 $(r=.80)$ and PHQ-9 $(r=.76)$ as well as significant negative relations between the SEDS-S, the SEHS-S $(r=-.38)$, and the BMSLSS $(r=$ -.56) (Dowdy et al., 2018).

Due to its original convenience sampling, the generalizability of findings for the SEDS-S was somewhat limited (Dowdy et al., 2018). Additionally, the original sample was confined to two schools in one region of California and was comprised primarily of Latino/a or Hispanic students (School 1 = 77.7\%; School $2=48.7 \%$ ). Dowdy and colleagues (2018) recommended further analysis of invariance across age, ethnicities, and genders. In response to these limitations, Furlong and colleagues (2021) have recently provided validation for the SEDS-S and its use among diverse student populations. This study used subsamples from 105,771 students from 113 California secondary schools across urban, suburban, and rural communities (Furlong et al., 2021). Measures of internal consistency were strong $(\alpha=.94)$ and similar to the original validation study ( $\alpha=.91$ ) (Dowdy et al., 2018). After reconfirming a good model fit for the single factor structure through CFA, measurement invariance tests were conducted at three levels (configural, metric, and scalar). Findings established measurement invariance across demographic items (gender, grade levels, Latinx status, and ethnicity groups) to support that psychological distress, as measured by the SEDS-S, was measured similarly across these demographic items.

\section{Research Design}

The primary goal of this study was to determine if scores from self-report measures of subjective well-being and psychological distress were significantly different as students reported more absenteeism. Variables and their subsequent measures were reviewed in the following sections. Questions regarding attendance on the CKS-SCM establish independent variables based 
on the amount of school students reported missing or skipping in the past 30 days and 12 months, respectively. Additional items on the CHKS-SCM identified demographic details for students for analysis of their relationship with attendance problems. Cross-sectional reports on the SEHS-S and SEDS-S measured students' subjective well-being and psychological distress, respectively.

\section{Independent Variables}

Groups established for the present study's independent variables (IV) were based on questions regarding attendance on the CHKS-SCM (Questions 19 and 21). A copy of questions 19 and 21 from the CHKS-SCM are included as Appendix A. These responses are similar to other questions about attendance (or truancy) from large nation-wide survey projects (e.g., National Comorbidity Survey: Adolescent Supplement [Kessler, 2001-2004]; Monitoring the Future national survey [Henry, 2007]; School Crime Supplement to the National Crime Victimization Survey [United States Department of Justice, 2007]; National Survey on Drug Use and Health [Vaughn et al., 2013]) used in prior studies related to the topic of school attendance. Despite their similar questions and response patterns, each of these surveys focused primarily on "truancy."

Question 19 (In the past 30 days, how often did you miss an entire day of school for any reason?) has four response options (did not miss any days of school, 1 day, 2 days, 3 or more days). Question 21 (During the past 12 months, about how many times did you skip school or cut classes?) has seven response options ( 0 times, 1-2 times, a few times, once a month, twice a month, once a week, more than once a week). These groups consisted of nominal (i.e., categorical) data. Additional demographic items gathered from the CHKS-SCM included students' grade levels, gender, sexual orientation, race/ethnicity, and socioeconomic status. These questions were used to consider their relationship with SRB. 


\section{Dependent Variables}

The present study maintained a holistic perspective of students' mental health by implementing a dual-factor model approach. Cross-sectional data was from the Social-Emotional Health Survey-Secondary (SEHS-S) and the Social-Emotional Distress Scale-Secondary (SEDSS) were used to measure subjective well-being and psychological distress, respectively. These measures and their respective constructs established dependent variables for the present study.

The SEHS-S, a measure of subjective well-being, is a 36-item scale with 4-point response options $(1=$ not at all true, $2=$ a little true, $3=$ pretty much true, $4=$ very much true $)$. Together, these questions amount to a total covitality score based on a range of raw scores from 36-144. The SEDS-S, a measure of psychological distress, is a 10-item measure using the same 4-point response scale $(1=$ not at all true, $2=$ a little true, $3=$ pretty much true, $4=$ very much true $)$. These questions amount to a total distress score with raw scores ranging from 10-40.

Together, these scales were designed for an integrated approach to screening for mental health with a dual-factor perspective (Suldo \& Shaffer, 2008). For Research Questions 3a, 3b, $4 \mathrm{a}$, and $4 \mathrm{~b}$, raw total scores were used when comparing groups. For the final Research Question 5, an Average Item Response (AIR) score was computed for each measure to assign students to appropriate groups from the dual-factor model based on their collective scores (Furlong et al., 2020; Suldo \& Shaffer, 2008). These final AIR scores range from 1-4 and can be separated into groups of low, average, and high scores (Furlong et al., 2020).

Figure 3 below presented each dual-factor group based on the outcomes from student reports of subjective well-being and psychological distress (Complete Mental Health, Symptomatic but Content, Vulnerable, and Troubled). Group assignment was based on established values from on the SEHS-S and SEDS-S, including low subjective well-being (AIR 
$=1.0-2.2)$ and low psychopathology $(\mathrm{AIR}=1.0-1.9)($ Vulnerable $)$; low subjective well-being (1.0-2.2) and high psychopathology (3.0-4.0) (Troubled); average to high subjective well-being (2.3-4.0) and high psychopathology (3.0-4.0) (Symptomatic but Content); and finally, average to high subjective well-being (2.3-4.0) and low psychopathology (1.0-2.9) (Complete Mental Health) (Furlong et al., 2020; Suldo \& Shaffer, 2008).

\section{Figure 3}

Group Assignment Based on Dual-Factor Model

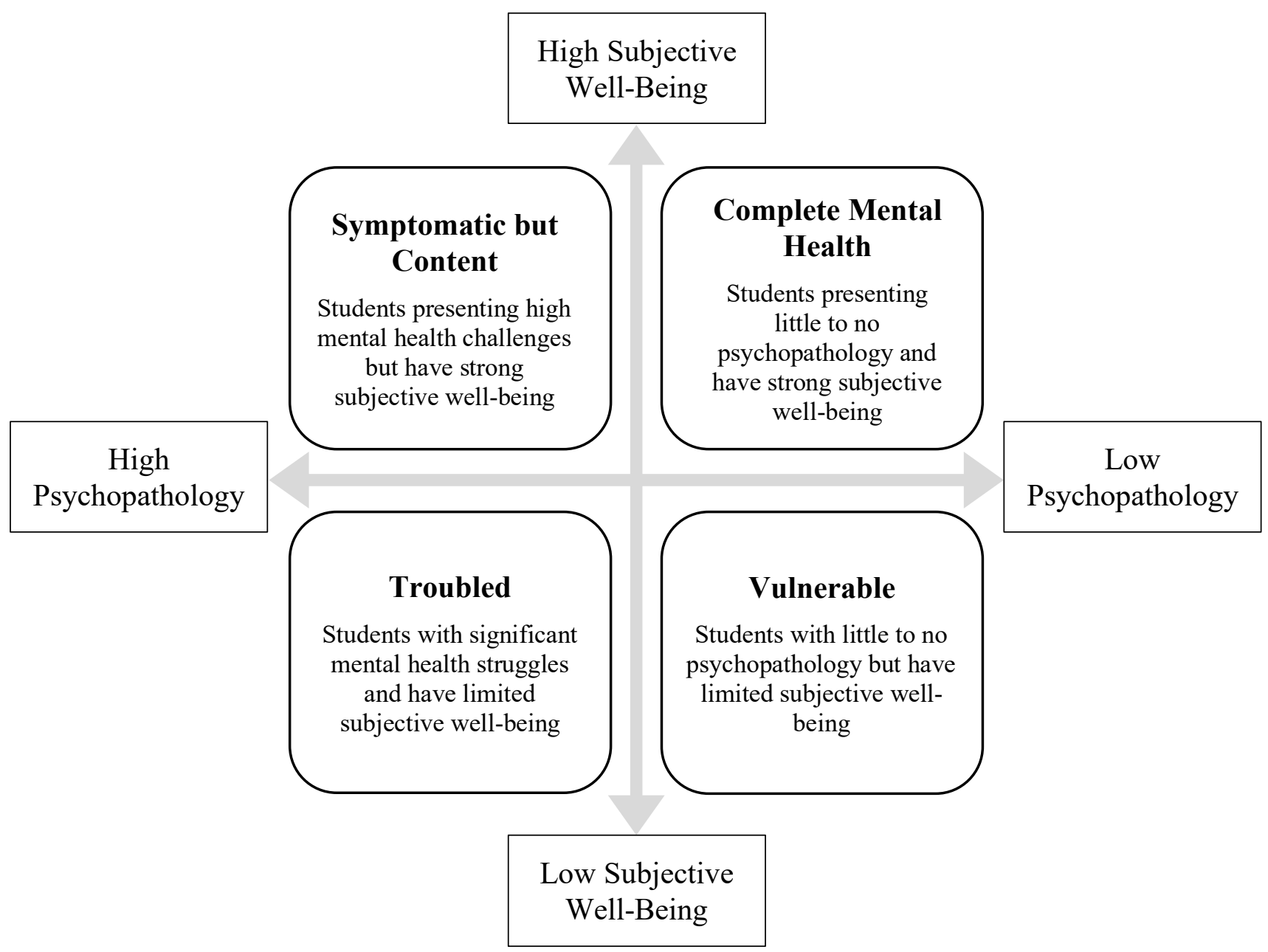




\section{Data Analysis Procedures}

Quantitative methods analyzed data using SPSS software, version 27. Initial frequency distributions computed for Questions 19 and 21 from the CHKS-SCM attendance questions reviewed the different responses to gather percentages of occurrence. Research Question 1 relied on this information regarding the prevalence of SRB measured by the CHKS-SCM compared to outcomes from other large datasets.

Using these separate groups based on attendance Questions 19 and 21 from the CHKSSCM, descriptive statistics provided demographic information for grade levels, gender identity, sexual orientation, race/ethnicity, and socioeconomic status). Cross-tabulation tables used rows of students' demographic information and columns with their attendance reports. Following this, Chi-square tests of independence were conducted for Research Questions $2 \mathrm{a}-2 \mathrm{~d}$ to determine if demographic factors, separately, had a significant relationship with attendance based on Questions 19 and 21. Effect sizes expressed the magnitude of these relationships as small, medium, or large.

For Research Questions 3a and 3b, mean scores of students' subjective well-being (SEHS-S) were compared across groups based on responses to Questions 19 and 21 from the CHKS-SCM. Next, for Research Questions 4a and 4b, these same procedures analyzed differences in reports of psychological distress (SEDS-S) for students based on their attendance reports. Due to necessary parametric assumptions not being met, the nonparametric Kruskal Wallis $H(\mathrm{~K}-\mathrm{W})$ test analyzed groups (see sections on preliminary analysis in Chapter 4). Further rank order and between-group analysis used pairwise comparisons of median scores to detect differences between group levels based on absenteeism levels. Each group was analyzed for statistical significance based on a $p$ value below .05 $(\alpha=.05)$. To better understand these 
differences, effect size calculations analyzed the magnitude between each group difference.

Figure 4 demonstrates a visual mapping of this process.

\section{Figure 4}

\section{Data Analysis Plan}

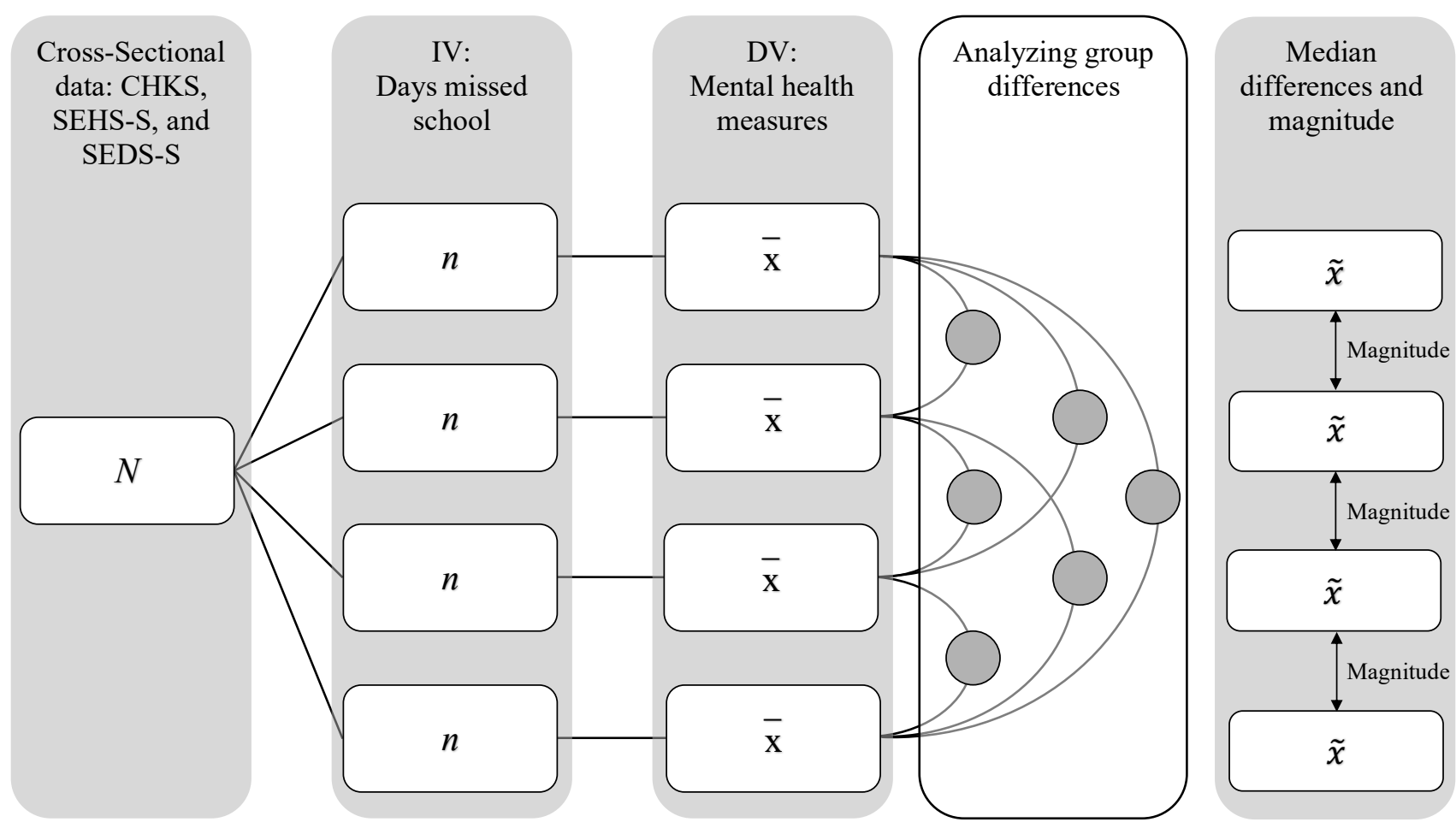

Finally, Research Question 5 utilized established dual-factor groups (Complete Mental Health, Symptomatic but Content, Vulnerable, and Troubled) based on AIR scores from the SEHS-S and SEDS-S. Cross-tabulation tables provided frequency distribution data for students from each dual-factor group and students presenting SRB (based on Questions 19 and 21 from the CHKS-SCM). A percentage of each dual-factor group represented students based on their responses to each attendance question. This indicated how many students from each dual-factor group presented SRB. Further descriptive information elaborated on the groups of students with SRB present in different dual-factor groups. 


\section{Methodology Descriptions: Analyzing Group Differences}

Effectively comparing groups has long been an integral process for research analyzing differences to better understand select aspects, or variables, within groups. This lengthy process involved establishing appropriate groups, determining levels of error, comparing differences, and discerning meaning from outcomes. By establishing a significant difference between groups, their relationship can be further examined to answer questions about their dynamics. Due to limitations within the data used in this study (i.e., non-normal distributions and heterogeneity of variance across group levels), nonparametric tests were primarily used to compare groups.

Effect size measures were used to describe the magnitude, or the degree, of a connection or difference. For instance, a study's findings may have resulted in statistical significance between select variables or groups, but no indication was readily provided for determining the impact that this relationship had (Salkind, 2007). Reporting measures of effect size have become an essential component of psychological research (Johnston et al., 2004).

Calculating an effect size includes three elements, standardization of the effect, specification of the direction an effect had, and independence from the sample size (Salkind, 2007). Ensuring these components allows for effect sizes to be generalized across multiple studies (e.g., meta-analyses) or groups. Effectively, effect sizes use mean (average) differences to compute the relationship between two variables. Other less common models rely on squaring the correlation coefficient outcomes or using odds ratios. Appropriate effect size measures should be determined by analyzing the nature of each sample's data and its underlying distribution (Lakens, 2013). 


\section{Pearson's Chi-Square Test}

When analyzing nominal (i.e., categorical) or ordinal data, the Pearson's Chi-square test $\left(\chi^{2}\right)$ was relied on (Sirkin, 2006). The use of critical value coefficients weres used for the outcome $\left(\chi^{2}\right)$ of this nonparametric test to determine whether a significant relationship exists between the observed or actual occurrences and expected occurrences of the groups. A larger difference between observed and expected values results in a larger Chi-square value.

If the final Chi-square value is equal to or greater than an established critical value from a critical values table, typically at the .05 level, a significant difference exists. A significant difference implies that a statistically significant association, not due to chance, exists between the groups on some level. To some extent, the complexity or amount of variables analyzed can influence probability outcomes. For example, the number of levels in each group may increase error or change probability outcomes (Salkind, 2010). Calculating the degrees of freedom ( $d f$ ) for each variable accounted for this:

$$
d f=(r-1)(c-1)
$$

In this formula, the $r$ value represents the number of rows in the table, while the $c$ value equals the number of columns.

\section{Chi-Square Assumptions}

Before computing a Chi-square test, specific criteria or assumptions must be met (Salkind, 2010). Primarily, variables should remain nominal (i.e., categorical) or ordinal. The sample must be randomly drawn from the population. Data must remain in raw form (i.e., no percentages or standardized scores), and variables must be independent from each other. The impact encountered in small sample sizes was unlikely in a large dataset like that of the current study. In general, expected frequencies should not be below five for more than $20 \%$ of the cells 
generated (Pagano, 2009); however, many view these specifications as arbitrary (Frey, 2018).

Results of a Chi-square are presented in cross-tabulation tables to assess differences between each group. When interpreting outcomes, results imply that the groups in a sample are significantly related in some way and should not be generalized to the greater population from which it came.

\section{Chi-Square Effect Size Calculations}

Effect size calculations measured the amount of difference, or magnitude, between each of the groups. Cramer's $\varphi$ (phi) was relied on for variables with dichotomous or two levels (male or female), and Cramer's $V$ was used when variables included more than two levels or categories (grades $7,8,9$, etc.). These effect sizes measure the association between variables to determine the level of their relationship based on a percentage of their variation; a result of 0.0 indicates no relationship is detected (Durlak, 2009). Further interpretation of these effect size results will follow the guidelines provided by Frey (2018), with 20-.29 representing a weak correlation or effect; $.30-.50$ representing a moderate effect; .50-.69 representing a strong effect; and .70-1.0 indicating a very strong effect.

\section{Analysis of Variance (ANOVA)}

Initially, a planned analysis using analysis of variance (ANOVA) tests was intended to compare scores for the SEHS-S and SEDS-S across groups based on attendance. However, necessary assumptions to run this parametric analysis were not met and a nonparametric alternative, the K-W test was used. A brief description of ANOVA procedures was provided here to further elaborate on standard procedures for statistically analyzing groups using quantitative methods; the K-W test relies on similar principles. 
An ANOVA is a popular tool in social sciences for experimental and non-experimental designs (Belhekar, 2016). Using an ANOVA relies on a general linear model, such that variables plotted along a path (i.e., vector) should resemble a straight line (linear path) to compare each group. The difference between each group is called between-group (explained) variance while the residual differences within individual groups is known as within-group (error) variance. The change that occurs in each IV, based on the DV, is referred to as the main effect whereas the combined interactions of IVs on the DV is called the interaction effect. The distribution of variables along a vector is tested to observe the overall fit of the data using $F$ statistics or an $F$ ratio. When measuring this variation, if between-group variance is larger than within-group variance, a statistically significant difference is found between the groups.

\section{ANOVA Assumptions}

To conduct an ANOVA, standard parametric procedures and assumptions must be accounted for and considered. By using three primary assumption criteria, researchers should ensure that their sample variables are appropriate for using the $F$-ratio statistic to measure variance. First, the DV should maintain normal distribution across each group or condition. ANOVAs are considered robust measures that are not strongly influenced by skewed data that can cause high or low tail distributions in a bell curve, referred to as high kurtosis (Salkind, 2010). However, platykurtic distributions, with thinner tails and a flattened top, can have significant effects on ANOVAs with small sample populations. Data used in the present study did not meet this assumption- details are provided in a section on Preliminary Analysis in Chapter 4.

Another assumption of ANOVA is that the samples have homogeneity of variance. Without ensuring consistent variation across groups, an ANOVA may result in inflated error 
measures. Data used in the present study did not meet this assumption- further details are provided in a section on Preliminary Analysis in Chapter 4.

Lastly, observed measures should be independent of each other (from separate groups or entities) and sampled randomly. In a repeated-measures design, however, this assumption of independence is not maintained, resulting in further assumptions (Salkind, 2007). Rather than homogeneity of variance, in a repeated-measures design, the requirement of variation in groups to be similar (not statistically significant) is called sphericity. In select study designs, nonparametric tests or transformations to data can be used to adjust for violations to these assumptions. The present study relied solely on cases and questions with independent data.

Measuring Normality. The assumption of normality seeks to ensure that data consists of a normal distribution in the shape of a classic bell curve (Salkind, 2010). The present study employed three methods to analyze normality across group levels, analyzing skewness and kurtosis values, using the Kolmogorov-Smirnov (K-S) test of normality $(\alpha=.05)$, and visual analysis of histogram graphs.

According to Muthén and Kaplan (1992), normality can be assessed according to the absolute value of one guideline, in which skewness and kurtosis scores within a range of +1.00 and -1.00 can be assumed to be evenly distributed. When using large sample sizes, recommendations also include the K-S test of normality (Orcan, 2020). A significant K-S test result $(p=<.05)$ implies that the groups were not normally distributed. Lastly, a visual analysis of histogram graphs using bar charts to demonstrate data distribution is an effective and simple way to confirm the distribution of variables (Salkind, 2010).

Measuring Homogeneity of Variance. When comparing groups or multiple levels within a group, having relatively similar distributions of variance presents another important 
assumption. This assumption compares whether the existing variance across groups was greater than that expected by chance (Lewis-Beck et al., 2004). Not meeting this assumption can lead to serious violations to parametric analyses and resulting $\mathrm{p}$ values (Salkind, 2010). A significant result from Levene's test of homogeneity of variance $(p=<.05)$ was used as an indication for significant variance across groups.

\section{Kruskal Wallis H Test}

The $\mathrm{K}-\mathrm{W}$ test is a nonparametric statistical procedure to substitute a one-way ANOVA if assumptions of group normality were violated (Salkind, 2010). Instead of an F statistic, this process identifies the median average of each group and compares for statistically significant differences. Rather than using their actual value, the $H$ test ranks each variable in order to compare their relative values; this outcome is often referred to as a one-way ANOVA on ranks. Further comparison of the $H$ statistic relies on Chi-square analyses to determine statistical significance across the group median rankings. Due to the K-W test being an omnibus test, subsequent pairwise comparisons are a critical aspect of the analysis that otherwise would not indicate which groups are significantly different from one another (Allen, 2017).

\section{Kruskal Wallis Assumptions}

A common assumption for this analysis includes the presence of one IV with three or more levels or conditions. Further, IVs in the analysis should remain independent from one another in the sample, and the DV should remain continuous (Allen, 2017). The Mann Whitney U test would analyze independent samples with IVs consisting of only two levels or conditions. Paired samples would use the Wilcoxon signed rank test (Salkind, 2010). 


\section{Kruskal Wallis Effect Size Calculations}

Traditional measures for effect size (e.g., Cohen's $d$ ) often rely on the assumptions of normality and homogeneity for its results to maintain validity (Johnston et al., 2004). Considering the heterogeneity in the present sample's groups, Glass' $\Delta($ Delta $)$ was calculated for comparing the association between groups following the $\mathrm{K}-\mathrm{W}$ test. This effect size measure uses the standard deviation from a control group to calculate standardized mean differences to account for what variance may be presented due to the lack of homogeneity (Salkind, 2007 \& 2010). Traditional interpretation for the outcomes of effect size described .2 as a small effect, .5 as a medium effect, and .8 as a large effect (Cohen, 1988).

\section{Research Questions, Hypotheses, and Analysis Plans}

Research Question 1: How do school attendance items from the current dataset compare to prevalence outcomes using other large datasets?

\section{Research Question 1 Variables}

Questions 19 and 21 from the CHKS-SCM were used to establish school attendance prevalence. Question 19 (In the past 30 days, how often did you miss an entire day of school for any reason?) includes four levels based on the possible responses (did not miss any days of school, 1 day, 2 days, 3 or more days). Question 21 (During the past 12 months, about how many times did you skip school or cut classes?) includes seven levels based on the possible responses (0 times, 1-2 times, a few times, once a month, twice a month, once a week, more than once a week).

\section{Research Question 1 Analysis Plan}

Data were split into files based on Questions 19 and 21, separately. Descriptive statistics were generated for frequency distributions for each of these groups and provided in separate 
tables. Percentages demonstrated the amount of school students reported missing based on Questions 19 and 21. Then, these percentages and descriptive findings were compared to other outcomes from studies of school attendance to determine if the CHKS-SCM resembled findings from other studies based on large survey datasets.

Research Questions 2a-2d: Are student self-reports of attendance consistent across demographic items?

\section{Research Question 2 Analysis Plan}

To understand the relationship between attendance and demographic backgrounds, Chisquare tests for independence analyzed select demographic items and Questions 19 and 21 on attendance from the CHKS-SCM. This procedure compared actual, or observed, scores from each group with expected values from groups if there was no association between them. A resulting significant difference would imply some level of association between the groups. This preliminary analysis acknowledged whether a significant relationship existed between attendance and select demographic factors. However, this analysis did not provide information regarding the direction of this relationship. Further analysis used effect size calculations and descriptive statistics to determine these outcomes.

Research Question 2a: Are significant differences observed in attendance groups based on grade level?

\section{Research Question 2a Variables}

The IV in this analysis included a demographic question regarding student grade levels from the CHKS-SCM. Responses to this item included grades 7 through 12. Questions 19 and 21 from the CHKS-SCM measured attendance as DVs in this analysis, separately. 


\section{Research Question 2a Analysis Plan}

Descriptive statistics provided frequency distributions for students' grade levels and attendance (based on Questions 19 and 21, separately). Crosstabulation tables and Chi-square tests analyzed these groups to determine if a significant association existed between attendance and grade levels. Effect size calculations and descriptive statistics further analyzed these results. Research Question 2b: Are significant differences observed in attendance groups based on gender or sexual orientation?

\section{Research Question 2b Variables}

The IV in this analysis included demographic questions from the CHKS-SCM regarding students' gender and sexual identity. A select question for gender included responses for male and female. A separate question for transgender students allowed for responses no, I am not transgender; yes, I am transgender; I am not sure if I am transgender; and students who declined to respond., These questions were analyzed separately to maintain independent case selection. Further, a question regarding sexual orientation included responses for straight (not gay), gay or lesbian, bisexual, I am not sure, something else, and decline to respond. Questions 19 and 21 from the CHKS-SCM measured attendance as DVs in this analysis, separately.

\section{Research Question 2b Analysis Plan}

Descriptive statistics provided frequency distributions for students' gender, sexual orientation, and attendance (based on Questions 19 and 21, separately). Using Chi-square tests, crosstabulation tables analyzed groups to determine if a significant association existed between attendance and gender or sexual orientation. Effect size calculations and descriptive statistics further analyzed these results. 
Research Question 2c: Are significant differences observed in attendance groups based on student race/ethnicity?

\section{Research Question 2c Variables}

The IV in this analysis included demographic questions regarding race/ethnicity from the CHKS-SCM. An initial question allowed students to answer yes/no to having Latinx or Hispanic origins. Additionally, a separate question allowed students to select their race/ethnicity (American Indian or Alaska Native, Asian, Black or African American, Native Hawaiian or Pacific Islander, White, or Mixed [two or more races]). These questions were each used, separately, to indicate race/ethnicity and maintain independent cases (students responding yes to being Hispanic/Latinx also frequently respond with Mixed (two or more races) as their race/ethnicity). Questions 19 and 21 from the CHKS-SCM measured attendance as a DV in this analysis, separately.

\section{Research Question 2c Analysis Plan}

Descriptive statistics provided frequency distributions for students' race/ethnicity and attendance (based on Questions 19 and 21, separately). Chi-square tests analyzed these groups in cross-tabulation tables to determine if a significant association existed between attendance and race/ethnicity. Effect size calculations and descriptive statistics further analyzed these results. Research Question 2d: Are significant differences observed in attendance groups based on socioeconomic status?

\section{Research Question 2d Variables}

The IV in this analysis included demographic questions serving as socioeconomic indicators from the CHKS-SCM. To fully consider socioeconomic status (SES), responses were from questions about parent education (did not finish high school, graduated from high school, 
attended college but did not finish, and graduated from college) and student eligibility for free or reduced-price lunch (yes or no) were selected. Students who responded don't know to either question were excluded from the analysis. Questions 19 and 21 from the CHKS-SCM measured attendance as a DV in this analysis, separately.

\section{Research Question 2d Analysis Plan}

Descriptive statistics provided frequency distributions for students' eligibility for free or reduced-price lunch, parent education level, and attendance (based on Questions 19 and 21, separately). Chi-square tests analyze groups using cross-tabulation tables to determine if a significant association existed between attendance and factors related to SES. Effect size calculations and descriptive statistics further analyzed these results.

Research Question 3a: Are there differences in self-reported subjective well-being measures (i.e., Social-Emotional Health Survey-Secondary [SEHS-S]) across students based on the amount of school they reported missing in the past 30 days (i.e., Question 19)?

\section{Research Question 3a Variables}

Responses for Question 19 from the CHKS-SCM ( 0 days, 1 day, 2 days, 3 or more days) will be used as groups to analyze differences in scores from the SEHS-S.

\section{Research Question 3a Analysis Plan}

Differences in scores from the SEHS-S across levels, or responses, from Question 29 from the CHKS-SCM were analyzed using the nonparametric K-W test. Group ranking and pairwise comparison for each group's median score were analyzed to detect significant differences between groups $(\alpha=.05)$. Lastly, effect sizes were computed using Glass' $\Delta$ to analyze the magnitude of difference between each group. 
Research Question 3b: Are there differences in self-reported subjective well-being measures (i.e., SEHS-S) across students based on the amount of school they reported skipping or cutting in the past 12 months (i.e., Question 21)?

\section{Research Question 3b Variables}

Responses for Question 21 from the CHKS-SCM (0 times, 1-2 times, a few times, once a month, twice a month, once a week, more than once a week) were used as groups to analyze differences in scores from the SEHS-S.

\section{Research Question 3b Analysis Plan}

The nonparametric K-W test analyzed differences in scores from the SEHS-S across or responses from Question 21. Group ranking and pairwise comparisons of each group's median score were analyzed to detect significant differences between each group $(\alpha=.05)$. Lastly, effect sizes were computed using Glass' $\Delta$ to analyze the magnitude of differences between each group. Research Question 4a: Are there differences in self-reported psychological distress measures (i.e., Social-Emotional Distress Scale-Secondary [SEDS-S]) across students based on the amount of school they reported missing in the past 30 days (i.e., Question 219)?

\section{Research Question 4a Variables}

Responses for Question 19 from the CHKS-SCM ( 0 days, 1 day, 2 days, 3 or more days) were used as groups to analyze differences in scores from the SEDS-S.

\section{Research Question 4a Analysis Plan}

The nonparametric K-W test was used to analyze differences in scores on the SEDS-S across responses from Question 19. Rank order for roup median scores and pairwise comparisons were analyzed to identify significant differences between each group $(\alpha=.05)$. Lastly, Glass' $\Delta$ was computed to gather effect size calculations to analyze the magnitude of these differences. 
Research Question 4b: Are there differences in self-reported psychological distress measures (i.e., SEDS-S) across students based on the amount of school they reported skipping or cutting in the past 12 months (i.e., Question 21)?

\section{Research Question 4b Variables}

Responses for Question 21 from the CHKS-SCM (0 times, 1-2 times, a few times, once a month, twice a month, once a week, more than once a week) were used as groups to analyze differences in scores from the SEDS-S.

\section{Research Question 4b Analysis Plan}

The nonparametric K-W test analyzed differences in SEDS-S scores for groups of students based on Question 21. Rank order and pairwise comparisons were used to detect significant differences between each group $(\alpha=.05)$. Lastly, effect size calculations were computed for Glass's $\Delta$ to analyze the magnitude of these outcomes.

Research Question 5: Using groups (a) Complete Mental Health, (b) Symptomatic but Content, (c) Vulnerable, and (d) Troubled from a dual-factor model approach, what percentage of students from each group presented with potential SRB?

\section{Research Question 5 Variables}

Raw scores from the SEHS-S and SEDS-S were converted into Average Item Response (AIR) scores by calculating overall mean scores (Furlong et al., 2020). For the SEHS-S, three subdomains (with three questions each) for each domain (belief-in-self, belief-in-others, emotional competence, engaged living) were added together and divided by nine, separately, to obtain AIR scores. Then, each domain's AIR score was added together and divided by four to gain the final SEHS-S covitality AIR score. The SEDS-S AIR score involved adding all ten items together and dividing by ten for its total AIR score. 
Furlong and colleagues (2020) have provided interpretive ranges with standard deviations for the AIR scores on the SEHS-S such that 1.0-2.2 are low, 2.3-3.5 are average (16-84\%), and 3.6-4.0 are high. Similarly, ranges for the SEDS-S include 1.0-1.9 as low, 2.0-3.0 as average (1684\%), and 3.1-4.0 as high (Furlong et al., 2020). Students were placed in respective groups based on their AIR score on the SEHS-S and SEDS-S- consistent with Suldo and Shaffer's (2008) dual-factor approach. Groups included Complete Mental Health (average to high subjective wellbeing and low psychopathology), Symptomatic but Content (average to high subjective wellbeing and high psychopathology), Vulnerable (low subjective well-being and low psychopathology), and Troubled (low subjective well-being and high psychopathology). Attendance questions 19 and 21 from the CHKS-SCM were used to measure attendance as a DV in this analysis.

\section{Research Question 5 Analysis Plan}

Cross-tabulation tables presented frequency distributions for each response to Questions 19 (missed 0 days, 1 day, 2 days, or 3 or more days in the past 30 days) and 21 (skipped 0 times, 1-2 times, a few times, once a month, twice a month, once a week, or more than once a week in the past 12 months) across the dual-factor groups (Complete Mental Health, Symptomatic but Content, Vulnerable, and Troubled). Descriptive statistics from these tables analyzed the distribution of students with SRB in groups based on the dual-factor model.

\section{Summary}

This study explored the differences in self-reported mental health measures for secondary students with different levels of absenteeism. Both subjective well-being and psychological distress were analyzed when measuring mental health to incorporate a dual-factor model perspective. To help understand the prevalence of SRB, the CHKS-SCM provided school-based 
data using questions related to attendance. Also, analysis using demographic items compared the present dataset to other findings regarding contextual factors that influence SRB (i.e., student grade levels, gender, race/ethnicity, socioeconomic status).

The present study focused on understanding the changing mental health of students with SRB. The classification, identification, and assessment of these students require a better understanding of their mental health changes as they miss or skip more school. The CHKS provided data readily available to most California schools and encouraged the use of schoolbased data to inform practical implications and research. This topic has lacked school-based informants and has previously relied primarily on clinical settings. 


\section{Chapter 4: Results}

In the following sections, the analysis plans from Chapter 3 were conducted and outlined. First, descriptive statistics were reviewed for each demographic item and both the SEHS-S and SEDS-S. Additionally, preliminary tests were conducted to determine if necessary parametric assumptions were met. Due to the violation of these assumptions, nonparametric tests were utilized throughout the analysis. Each research question has a primary analysis section with results presented for each question consecutively.

\section{Preliminary Analysis}

Research Question 1 involved an initial review of prevalence data compared with separate, prior studies. Research Questions 2a-2d analyzed the association between attendance and select demographic items. Chi-square tests detected if a significant relationship was present. For Research Questions 3 and 4, this study compared groups of students based on their attendance and mental health using Kruskal Wallis $H$ tests. This process compared measures outcomes from subjective well-being and psychological distress separately to determine if there was a difference in students with higher levels of absenteeism. Finally, an analysis of descriptive statistics for the dual-factor model groups based on students' mental health revealed what percentage of vulnerable (at-risk) and troubled youth presented with potential SRB struggles. Chi-square tests determined if a significant relationship existed between students' absenteeism and the dual-factor model groups.

\section{Descriptive Statistics}

Overall demographic items are reviewed from the CHKS-SCM to help understand the sample of students in the present dataset. The current data consists of responses from 107,125 students in grades 7-12 from 249 secondary schools across California from the 2017-18 and 
2018-19 school years. Overall, this sample consisted of 51.4\% females, $48.1 \%$ Hispanic/Latinx, $46.8 \%$ students with parents who graduated with a college degree, and $40.4 \%$ students eligible for free or reduced-price lunch at school. Table 1 provides further demographic details alongside mean scores from the SEHS-S and SEDS-S. The SEHS-S includes scores from 36-144, and the SEDS-S has a range from 10-40. Missing responses may cause some variation in frequency outcomes $(n)$ for the SEHS-S and SEDS-S outcomes.

\section{Table 1}

Demographic Information and Corresponding SEHS-S and SEDS-S Mean Scores

\begin{tabular}{|c|c|c|c|c|c|}
\hline Demographic Items & $\begin{array}{c}n \\
(\%)\end{array}$ & $\begin{array}{c}\text { SEHS-S } \\
\text { Mean Score } \\
(n)\end{array}$ & $\begin{array}{c}\text { SEHS-S Std. } \\
\text { Deviation }\end{array}$ & $\begin{array}{c}\text { SEDS-S } \\
\text { Mean Score } \\
(n)\end{array}$ & $\begin{array}{c}\text { SEDS-S Std. } \\
\text { Deviation }\end{array}$ \\
\hline
\end{tabular}

\section{Grades}

\begin{tabular}{rccccc} 
Seventh & 27786 & 109.72 & 21.07 & 18.49 & \\
& $(25.9 \%)$ & $(23653)$ & & $(26402)$ & \\
Eighth & 5710 & 106.75 & 21.78 & 19.28 & 8.47 \\
& $(5.3 \%)$ & $(4969)$ & & $(5450)$ & \\
Ninth & 29635 & 105.06 & \multirow{2}{*}{21.38} & 20.12 & 8.72 \\
& $(27.7 \%)$ & $(25957)$ & & $(28470)$ & \\
Tenth & 10529 & 104.04 & & 20.81 & 8.80 \\
& $(9.8 \%)$ & $(9392)$ & 20.55 & $(10177)$ & \\
Eleventh & 25112 & 104.61 & & 21.16 & \\
& $(23.4 \%)$ & $(22579)$ & 20.22 & $(24302)$ & \\
Twelfth & 8353 & 105.71 & & 20.90 & \\
& $(7.8 \%)$ & $(7584)$ & 19.93 & $(8096)$ &
\end{tabular}

\section{Gender}

$\begin{array}{cccccc}\text { Male } & 51320 & 106.53 & 21.42 & 17.78 & 7.72 \\ & (48.6 \%) & (45109) & & (49366) & \\ \text { Female } & 54214 & 105.86 & 22.16 & 8.10\end{array}$




\begin{tabular}{|c|c|c|c|c|c|}
\hline Demographic Items & $\begin{array}{c}n \\
(\%)\end{array}$ & $\begin{array}{c}\text { SEHS-S } \\
\text { Mean Score } \\
(n)\end{array}$ & $\begin{array}{c}\text { SEHS-S Std. } \\
\text { Deviation }\end{array}$ & $\begin{array}{c}\text { SEDS-S } \\
\text { Mean Score } \\
(n)\end{array}$ & $\begin{array}{c}\text { SEDS-S Std. } \\
\text { Deviation }\end{array}$ \\
\hline
\end{tabular}

Transgender

$\begin{array}{rccccc}\text { Not transgender } & 98261 & 106.64 & 20.64 & 19.89 & 8.55 \\ & (95.3 \%) & (86614) & & (94457) & \\ \text { Transgender } & 758 & 87.35 & 23.52 & 27.63 & 10.08 \\ & (0.7 \%) & (633) & & (726) & \\ \text { I am not sure if I am } & 1552 & 94.83 & 22.22 & 25.13 & 9.45 \\ \text { transgender } & (1.5 \%) & (1328) & & (1486) & \\ & 2530 & 98.62 & & 20.72 & \\ \text { Declined to respond } & (2.5 \%) & (2110) & 24.70 & (2403) & 9.20\end{array}$

\section{Race/Ethnicity}

\begin{tabular}{|c|c|c|c|c|c|}
\hline Hispanic/Latinx & $\begin{array}{c}51205 \\
(48.1 \%)\end{array}$ & $\begin{array}{l}104.47 \\
(44083)\end{array}$ & 21.39 & $\begin{array}{c}19.68 \\
(48790)\end{array}$ & 8.54 \\
\hline Non-Hispanic/Latinx & $\begin{array}{c}55155 \\
(51.9 \%)\end{array}$ & $\begin{array}{l}107.67 \\
(49460)\end{array}$ & 20.44 & $\begin{array}{c}20.38 \\
(53414)\end{array}$ & 8.71 \\
\hline $\begin{array}{r}\text { American Indian or Alaska } \\
\text { Native }\end{array}$ & $\begin{array}{c}3072 \\
(3.1 \%)\end{array}$ & $\begin{array}{l}104.27 \\
(2600)\end{array}$ & 22.73 & $\begin{array}{l}19.26 \\
(2926)\end{array}$ & 8.59 \\
\hline Asian & $\begin{array}{c}14593 \\
(14.8 \%)\end{array}$ & $\begin{array}{l}106.63 \\
(13210)\end{array}$ & 19.85 & $\begin{array}{c}19.9 \\
(14154)\end{array}$ & 8.22 \\
\hline Black or African American & $\begin{array}{c}2966 \\
(3.0 \%)\end{array}$ & $\begin{array}{l}106.10 \\
(2484)\end{array}$ & 23.45 & $\begin{array}{l}19.14 \\
(2804)\end{array}$ & 8.52 \\
\hline $\begin{array}{r}\text { Native Hawaiian or Pacific } \\
\text { Islander }\end{array}$ & $\begin{array}{c}1474 \\
(1.5 \%)\end{array}$ & $\begin{array}{l}106.53 \\
(1283)\end{array}$ & 21.02 & $\begin{array}{l}20.97 \\
(1415)\end{array}$ & 8.69 \\
\hline White & $\begin{array}{c}34101 \\
(34.6 \%)\end{array}$ & $\begin{array}{l}108.02 \\
(30652)\end{array}$ & 20.38 & $\begin{array}{c}20.59 \\
(33026)\end{array}$ & 8.85 \\
\hline Mixed (two or more) Races & $\begin{array}{c}42461 \\
(43.0 \%)\end{array}$ & $\begin{array}{l}104.95 \\
(36708)\end{array}$ & 21.51 & $\begin{array}{c}19.78 \\
(40572)\end{array}$ & 8.63 \\
\hline $\begin{array}{l}\text { ghest Level of Education } \\
\text { Parents or Guardians a }\end{array}$ & & & & & \\
\hline Did not finish high school & $\begin{array}{c}12517 \\
(11.7 \%)\end{array}$ & $\begin{array}{l}101.62 \\
(10690)\end{array}$ & 21.42 & $\begin{array}{c}20.12 \\
(11878)\end{array}$ & 8.57 \\
\hline Graduated from high school & $\begin{array}{c}14773 \\
(13.8 \%)\end{array}$ & $\begin{array}{l}103.79 \\
(12764)\end{array}$ & 21.68 & $\begin{array}{c}20.10 \\
(14141)\end{array}$ & 8.73 \\
\hline
\end{tabular}




\begin{tabular}{|c|c|c|c|c|c|}
\hline Demographic Items & $\begin{array}{l}n \\
(\%)\end{array}$ & $\begin{array}{c}\text { SEHS-S } \\
\text { Mean Score } \\
(n)\end{array}$ & $\begin{array}{l}\text { SEHS-S Std. } \\
\text { Deviation }\end{array}$ & $\begin{array}{c}\text { SEDS-S } \\
\text { Mean Score } \\
(n)\end{array}$ & $\begin{array}{l}\text { SEDS-S Std. } \\
\text { Deviation }\end{array}$ \\
\hline $\begin{array}{l}\text { Attended college but did not } \\
\text { complete a four-year degree }\end{array}$ & $\begin{array}{c}12810 \\
(12.0 \%)\end{array}$ & $\begin{array}{l}105.33 \\
(11266)\end{array}$ & 20.48 & $\begin{array}{c}21.17 \\
(12300)\end{array}$ & 8.94 \\
\hline Graduated college & $\begin{array}{c}49950 \\
(46.8 \%)\end{array}$ & $\begin{array}{l}109.05 \\
(45097)\end{array}$ & 19.90 & $\begin{array}{c}20.26 \\
(48400)\end{array}$ & 8.67 \\
\hline \multicolumn{6}{|l|}{$\begin{array}{l}\text { Eligible for Free or Reduced- } \\
\text { price Lunch }^{\text {a }}\end{array}$} \\
\hline Yes/Eligible & $\begin{array}{c}43024 \\
(40.4 \%)\end{array}$ & $\begin{array}{l}104.37 \\
(36991)\end{array}$ & 21.48 & $\begin{array}{c}19.67 \\
(41043)\end{array}$ & 8.55 \\
\hline No/Ineligible & $\begin{array}{c}50090 \\
(47.0 \%)\end{array}$ & $\begin{array}{l}107.96 \\
(45031)\end{array}$ & 20.15 & $\begin{array}{c}20.52 \\
(48525)\end{array}$ & 8.75 \\
\hline
\end{tabular}

${ }^{\text {a }}$ Students responding don't know were excluded from analysis

\section{Testing for Parametric Assumptions}

An ANOVA is traditionally used to compare groups to determine if a significant difference exists. However, this type of parametric analysis must maintain certain assumptions. To conduct an ANOVA, the dependent variable(s) (i.e., subjective well-being and psychological distress) must remain normally distributed across each level of the independent variable group (i.e., absenteeism). The present study used measures of skewness and kurtosis, the KolmogorovSmirnov (K-S) test of normality, and observations of histogram tables to determine if normality was present. Levene's test analyzed variance across each group to decide if they were significantly different when measuring homogeneity.

\section{SEHS-S Scores for Question 19 Responses}

Frequency distributions for Question $19(n=93,761)$, based on responses from the SEHS-S, are presented with skewness and kurtosis outcomes in Appendix F. Outcomes for skewness and kurtosis for each group level complied with the absolute value of one guideline 
and are within a range of +1.00 to -1.00 . An appropriately shaped bell curve demonstrated a normal distribution. However, each group of responses for Question 19 showed significance $(p=$ $<.001$ ) at a .05 alpha level on the K-S test of normality- despite Lilliefors Significance Correction. These results suggested that the distribution of each group of responses may be significantly different than the other. Histogram charts further examined the distribution of each group of responses to Question 19 and responses from the SEHS-S. Despite some skewness, these histograms supported a normal distribution. Each histogram chart is included in Appendix F.

When testing the assumption for homogeneity of variance across each level of Question 19 , Levene's test resulted in significant findings at a $p<.05$ level: $F(3,93,757)=32.24, p=$ $<.001$. Significant findings continued to result when comparing covariance across each level based on median and adjusted or trimmed median and means, respectively. Consequently, the assumption of homogeneity was unmet, and alternative, nonparametric tests were conducted to measure the difference between students' scores on the SEHS-S based on Question 19.

\section{SEHS-S Scores for Question 21 Responses}

Frequency distributions for Question $21(n=93,941)$, based on responses from the SEHS-S, were presented with skewness and kurtosis outcomes in Appendix G. Outcomes for skewness and kurtosis for each group level complied with the absolute value of one guideline and were within a range of +1.00 to -1.00 . Results supported an appropriate bell-shaped, normal distribution. However, each group of responses to Question 21, except for students reporting once a week $(p=.11)$, showed significance $(p=<.001)$ at a .05 alpha level on the K-S test of normality- despite Lilliefors Significance Correction. These findings suggested that the distribution of each group of responses was significantly different than the other. Histogram 
charts provided a further examination of the distribution for groups of responses to Question 21 and responses from the SEHS-S. Each histogram chart is included in Appendix G. A normal distribution was supported despite some skewness. Sample sizes became drastically smaller for students who reported skipping school more than once a month (refer to frequency distribution table in Appendix G for further details).

When testing the assumption for homogeneity of variance across each level of Question 21, Levene's test resulted in significant findings at a $p<.05$ level: $F(6,93,934)=11.74, p=$ $<.001$. Significant findings continued to result when comparing covariance across each level based on median and adjusted or trimmed median and means, respectively. Consequently, the assumption of homogeneity was unmet.

\section{SEDS-S Scores for Question 19 Responses}

Frequency distributions for Question $19(n=102,459)$, based on responses from the SEDS-S, were presented with skewness and kurtosis outcomes in Appendix H. Outcomes for skewness and kurtosis for each group level complied with the absolute value of one guideline and were within a range of +1.00 to -1.00 . However, skewness and kurtosis levels for the SEDSS appeared to be much greater than the distribution for scores from the SEHS-S. Findings supported an appropriate bell-shaped, normal distribution. Each group of responses to Question 19 showed significance $(p=.000)$ at a .05 alpha level on the K-S test of normality- despite Lilliefors Significance Correction. These results suggested that the distribution for each group of responses was significantly different than the other. Histogram charts further examined the distribution for each group of responses to Question 19 and responses from the SEHS-S. Each histogram chart is included in Appendix H. Skewness was visibly apparent, particularly for students who responded they missed 0 days. A flat-topped bell curve appeared throughout each 
group level. These observations, coupled with relatively increased skewness and kurtosis scores and significant results from the K-S tests, did not support a normal distribution.

When testing the assumption for homogeneity of variance across each level of Question 19, Levene's test resulted in significant findings at a $p<.05$ level: $F(3,102,455)=195.73, p=$ $<.001$. Significant findings continued to result when comparing covariance across each level based on median and adjusted or trimmed median and means, respectively. Consequently, the assumption of homogeneity was unmet.

\section{SEDS-S Scores for Question 21 Responses}

Frequency distributions for Question $21(n=102,662)$, based on responses from the SEDS-S, were presented with skewness and kurtosis outcomes in Appendix I. Outcomes for skewness and kurtosis for each group level did not comply with the absolute value of one guideline due to select responses to Question 21 presenting high, negative kurtosis scores (once a month, twice a month, once a week, and more than once a week). Further signs of uneven distributions were significant $(p<.05)$ for each group of responses on the K-S test of normality. Additional analysis of normal distribution relied on histogram charts provided in Appendix I. Sample sizes became drastically smaller for students with responses once a month, twice a month, once a week, more than once a week (refer to frequency distribution table in Appendix I for further details). Skewness for students who responded 0 times is visibly apparent. The following group levels maintained a flat-topped distribution emphasized by the negative kurtosis scores greater than -1.00 . Based on the observation of these charts, in addition to increased kurtosis scores and significant results from the K-S test, assumptions for normality do not appear to be supported. 
Levene's test resulted in significant findings at a $p<.05$ level: $F(6,102,655)=44.39, p=$ $<.001$ when testing for the assumption of homogeneity of variance for responses to Question 21, Significant findings continued to result when comparing covariance across each level based on median and adjusted or trimmed median and means, respectively. Consequently, the assumption of homogeneity was unmet.

\section{Internal Consistency Reliability for the SEHS-S and SEDS-S}

Scores on both the SEHS-S and SEDS-S demonstrated unequal variance across Questions 19 and 21. These findings were unsurprising considering the heterogeneity consistent with SRB. Unfortunately, this prevented parametric analysis from being used, as well as further posthoc tests. However, the reliability of scores from both screeners demonstrated exceptionally strong internal consistency based on Cronbach's alpha. This analysis, presented in Table 2, suggested that both the SEHS-S and SEDS-S, separately, had consistent measures even as students reported missing or skipping school more frequently. Together, the SEHS-S and SEDS-S provided a comprehensive approach to measuring subjective well-being and psychological distress in students with SRB.

\section{Table 2}

Internal Consistency for SEHS-S and SEDS-S Scores across Questions 19 and 21

\begin{tabular}{lcc}
\hline $\begin{array}{l}\text { Question 19: In the past 30 days, how often did you miss an } \\
\text { entire day of school for any reason? }\end{array}$ & $\begin{array}{c}\text { SEHS-S Cronbach's } \\
\text { Alpha }\end{array}$ & $\begin{array}{c}\text { SEDS-S Cronbach's } \\
\text { Alpha }\end{array}$ \\
\hline O days & .953 & .934 \\
I day & .952 & .936 \\
2 days & .950 & .939 \\
3 or more days & .952 & .940 \\
& & Table continued... \\
\hline
\end{tabular}




\begin{tabular}{lcc}
\hline $\begin{array}{l}\text { Question 21: During the past } 12 \text { months, about how many times } \\
\text { did you skip school or cut classes? }\end{array}$ & $\begin{array}{c}\text { SEHS-S Cronbach's } \\
\text { Alpha }\end{array}$ & Alpha \\
\hline O times & .952 & .936 \\
l-2 times & .951 & .936 \\
A few times & .950 & .936 \\
Once a month & .950 & .945 \\
Twice a month & .949 & .940 \\
Once a week & .943 & .945 \\
More than once a week & .953 & .948 \\
\hline
\end{tabular}

\section{Primary Analysis}

Research Question 1: How do school attendance items in the current data compare to prevalence outcomes using other large datasets?

\section{Analysis Results}

Table 3 below shows the frequency distribution of data for Question $19(n=106,651)$ and Question $21(n=106,857)$ from the CHKS-SCM. These included students who reported missing an entire day(s) of school in the past 30 days (Question 19) and those who reported skipping or cutting school in the past 12 months (Question 21). Nearly half (48\%) of the sample from Question 19 reported missing 0 days of school in the past 30 days. Students reporting to have missed 3 or more days, $13.5 \%$, pose the risk of missing approximately $15 \%$ of the available school days and demonstrated a pattern indicative of SRB.

On the other hand, even more students (74.7\%) from Question 21 reported having skipped or cut school 0 times over the past 12 months, while $12.2 \%$ reported skipping 1-2 times and $8.3 \%$ reported skipping only a few times. After excluding students who reported skipping 0 , 1-2, and a few times on Question 21, the remaining responses from students who reported skipping more frequently (once a month, twice a month, once a week, and more than once a 
week) represented $4.5 \%$ of the sample. Students who reported skipping or cutting school more than once a month (i.e., twice a month, once a week, or more than once a week, 3.6\%) had the potential of skipping between $11 \%$ and approximately $>40 \%$ of available school- assuming entire days of school were being skipped or cut. These responses represented students with potential patterns of risk for SRB.

\section{Table 3}

Frequency Distribution for Attendance Questions 19 and 21

\begin{tabular}{lcc}
\hline Question 19: In the past 30 days, how often did you & $n$ & $\%$ \\
miss an entire day of school for any reason? & 51420 & 48.0 \\
\hline O days & 24534 & 22.9 \\
1 day & 16279 & 15.2 \\
2 days & 14418 & 13.5 \\
3 days or more & 106651 & 99.6 \\
Total & & $\%$ \\
\hline Question 21: During the past 12 months, about how & $n$ & 74.7 \\
many times did you skip school or cut classes? & 80017 & 12.2 \\
\hline O times & 13017 & 8.3 \\
l-2 times & 8870 & 0.9 \\
A few times & 977 & 1.2 \\
Once a month & 1335 & 99.7 \\
Twice a month & 908 & 0.8 \\
Once a week & 1733 & 1.6 \\
More than once a week & 106857 & \\
Total & & \\
\hline
\end{tabular}

Based on responses from Questions 19 and 21 from the CHKS-SCM, groups of students who reported missing 3 or more days in the past 30 days (13.5\%) or skipping or cutting twice a 
month, once a week, and more than once a week (4.5\%), presented with the potential to miss approximately $15 \%$ or between $11-40 \%$ of available school, respectively. This spectrum of absence was similar to, yet, more narrowed than the $5-28 \%$ prevalence findings commonly cited for SRB (Heyne et al., 2019; Kearney, 2001; Lyon \& Cotler, 2007).

Research Question 2: Do student reports of attendance have a significant relationship with demographic variables?

Research Question 2a: Is there a significant relationship between attendance and grade level?

\section{Analysis Results}

Cross-tabulation tables with frequency data for student grade levels and responses to attendance Questions 19 and 21 are provided in Appendix J. Based on this data, students who reported missing 3 or more days of school in the past 30 days increased with each grade level (grade $7=11.7 \%$; grade $8=12.5 \%$; grade $9=12.6 \%$; grade $10=13.8 \%$; grade $11=15.2 \%$; grade $12=17.9 \%$ ). A similar trend was witnessed with more students from higher grade levels reporting to skip school or cut class more frequently in the past 12 months (e.g., reports of skipping school or cutting class for twice a month and more [twice a month, once a week, and more than once a week] increased with each grade level: grade $7=1.6 \%$; grade $8=1.8 \%$; grade $9=2.9 \%$; grade $10=3.9 \%$; grade $11=5.7 \%$; and grade $12=8.2 \%$ )

To analyze the association between grade levels and self-reported attendance for Question 19, a Chi-square test for independence was used to compare observed results to expected groups with no association; significant results suggest an association exists between the items. Results from this Chi-square analysis indicated a significant association between grade levels and attendance responses for Question $19, \chi^{2}(15, n=106,651)=997.340, p=<.001$, Cramer's $V=.056$. When analyzing the association between grade levels and self-reported 
attendance for Question 21, similar results were found, $\chi^{2}(30, n=106,857)=2,824.578, p=$ .000 , Cramer's $V=.073$. Overall, little statistical effect size was found, although Question 21 indicated a relatively greater magnitude of effect between grade levels and students who reported skipping or cutting school in the past 12 months.

Research Question 2b: Is there a significant association between attendance and gender or sexual orientation?

\section{Analysis Results}

Cross-tabulation tables with frequency data for students' gender and responses to attendance Questions 19 and 21 are provided in Appendix K. Based on self-reported attendance data from Question 19, female students reported higher rates of missing 3 or more days of school in the past 30 days $(15.1 \%)$ than males $(11.8 \%)$. Similarly, reports of skipping school or cutting class in the past 12 months indicated slightly higher reports from females across all responses (12 times, a few times, twice a month, once a week, and more than once a week) except for students reporting once a month ( $0.9 \%$ of both males and females). Further, $21.3 \%$ of students who identified as transgender reported missing 3 or more days of school as compared to students who reported no, I am not transgender (13.2\%), I am not sure if I am transgender (15.6\%), or those who declined to respond (15.3\%). This trend continued to be observed in Question 21 by transgender students reporting higher rates of skipping or cutting school across each response level when compared to other response options.

Cross-tabulation tables for sexual orientation (straight [not gay], gay or lesbian, bisexual, I am not sure, something else, and decline to respond) and responses to Questions 19 and 21, separately, are provided in Appendix K. Based on this data, gay or lesbian (18.4\%) and bisexual (18.4\%) students presented the highest frequency in Question 19 for missing 3 or more days of 
school in the past 30 days. Students who reported straight (not gay) (12.9\%) or decline to respond (12.9\%) represented the lowest frequency. Based on responses from Question 21, a similar trend continued with gay or lesbian and bisexual students giving higher reports of skipping school or cutting class more often than other sexual orientation responses.

To analyze the association between gender or sexual orientation and self-reported attendance from Questions 19 and 21, Chi-square tests for independence were used to compare observed results to groups with no expected association. Significant results suggest some association between the items. Results from a Chi-square analysis indicated significant association existed between gender and attendance responses for Question 19, $\chi^{2}(3, n=$ $105,074)=632.676, p=<.001$, Cramer's $V=.078$. When analyzing the association between gender and self-reported attendance for Question 21, similar results were found, $\chi^{2}(6, n=$ $105,274)=55.949, p=<.001$, Cramer's $V=.023$. Small effect sizes were calculated for both questions. Similarly, significant results were found across Questions $19\left(\chi^{2}[9, n=102,673]=\right.$ 99.06, $p=<.001$, Cramer's $V=.018)$ and $21\left(\chi^{2}[18, n=102,853]=154.46, p=<.001\right.$, Cramer's $V=.022$ ) for items related to transgender identification (no, I am not transgender; yes, I am transgender; I am not sure if I am transgender; or decline to respond).

When analyzing the association between sexual orientation and self-reported attendance, Chi-square tests for independence were conducted again using responses to Questions 19 and 21. Results from these Chi-square analyses indicated a significant association between sexual orientation and attendance responses for Question $19, \chi^{2}(15, n=102,659)=340.932, p=<.001$, Cramer's $V=.033$, and Question 21, $\chi^{2}(30, n=102,840)=420.411, p=<.001$, Cramer's $V=$ .029. These effect size outcomes were also small. 
Research Question 2c: Are significant differences observed in attendance groups based on student racelethnicity?

\section{Analysis Results}

Cross-tabulation tables with frequency data for students who did or did not identify as Hispanic/Latinx (yes/no) and responses to attendance Questions 19 and 21 are provided in Appendix L. Based on this data, Hispanic/Latinx students reported missing 3 or more days of school more often (15\%) than non-Hispanic/Latinx students (12.2\%). This trend was consistent across each response option for Question 21, with Hispanic/Latinx students reporting to skip or cut school more often- with the exception of students reporting to skip once a month (equal representation of $0.9 \%$ for both Hispanic/Latinx and non-Hispanic/Latinx). To analyze the overall relationship between attendance and students identifying as Hispanic/Latinx or otherwise, Chi-square tests were conducted. Results from these analyses were significant for both Questions $19\left(\chi^{2}[3, n=105,902]=410.35, p=<.001\right.$, Cramer's $\left.V=.062\right)$ and $21\left(\chi^{2}[6, n=106,103]=\right.$ 413.57, $p=<.001$, Cramer's $V=.062$ ). Effect sizes for these analyses remained small.

Cross-tabulation tables with frequency data for students' race/ethnicity and responses to attendance Questions 19 and 21 are included in Appendix L. Based on this data, Asian students reported missing school 1 day, 2 days, or 3 or more days less than any other race/ethnicity. American Indian or Alaska Native and Black or African American students equally represented reports for missing 3 or more days of school (16.2\%). Mixed (two or more) races and Native Hawaiian or Pacific Islander students were also equally represented and next likely to report missing 3 or more days (15.2\%). Nearly $14 \%$ of white students reported missing 3 or more days of school. These trends remained consistent across responses for Question 21, regarding student reports of skipping or cutting school in the past 12 months. 
To analyze the association between race/ethnicity and self-reported attendance for Questions 19 and 21, Chi-square tests for independence were used. Significant results suggest some association between the items. Results from these Chi-square analyses indicated a significant association between race/ethnicity and attendance responses for Question $19, \chi^{2}(15$, $n=98,264)=2339.61, p=.000$, Cramer's $V=.089$ and Question 21, $\chi^{2}(30, n=98,443)=$ 446.30, $p=<.001$, Cramer's $V=.03$. Again, these effect sizes remained small; however, compared to the other analyses of demographic factors, students' race/ethnicity and reports of missing school in the past 30 days had the largest relative effect size.

Research Question 2d: Is there a significant association between attendance and socioeconomic status?

\section{Analysis Results}

Cross-tabulation tables with frequency data for parent education levels and responses to attendance Questions 19 and 21 are included in Appendix M. Based on this data, an increase was observed in reports for missing 3 or more days of school in the past 30 days as parent education decreased (did not finish high school [16.6\%], graduated from high school [16.1\%], attended college bud did not complete a four-year degree [16\%], graduated from college [11.2\%]). Analysis of data for Question 21 resulted in similar outcomes regarding skipping or cutting school in the past 12 months. These reports indicated students whose parents graduated from college skipped school less frequently than all other parent education groups (did not finish high school, graduated from high school, and attended college but did not complete a four-year degree) across each response option (0 times, 1-2 times, a few times, once a month, twice a month, once a week, and more than once a week). 
To analyze the association between parent education levels and attendance using Questions 19 and 21, Chi-square tests for independence were used. Results from this analysis indicated a significant association between parent education levels and student self-reported attendance from Question 19, $\chi^{2}(12, n=106,222)=976.361, p=<.001$, Cramer's $V=.055$ and Question 21, $\chi^{2}(24, n=106,422)=999.584, p=<.001$, Cramer's $V=.048$. Similar to previous results, effect size calculations demonstrated small statistical magnitude regarding the relationship between parent education level and student attendance.

Using cross-tabulation tables, frequency data for students' eligibility for free or reducedprice lunch (yes or no) and attendance Questions 19 and 21 are provided in Appendix M. Based on this data, a higher percentage of students who qualified for free and reduced-price lunches $(15.2 \%)$ reported missing 3 or more days of school in the past 30 days than students who were not eligible (12\%). Similar results were found for Question 21, indicating that students eligible for free or reduced-price lunch more often reported skipping school 1-2 times, a few times, once a week, and more than once a week.

To analyze the association between students eligible for free and reduced-price lunch and their self-reported attendance, Chi-square tests for independence were used to compare observed results to groups with no expected association. Significant results suggest an association between the items. Results from this Chi-square analysis indicated a significant association between eligibility for free or reduced-price lunch and attendance responses for Question $19, \chi^{2}(3, n=$ $92,746)=301.922, p=<.001$, Cramer's $V=.057$. When analyzing the association between eligibility for free or reduced-price lunch and self-reported attendance for Question 21, similar results were found, $\chi^{2}(6, n=92,907)=298.268, p=<.001$, Cramer's $V=.057$. These effect size calculations, similar to each other demographic factor, remained statistically small. 
Research Question 3a: Are there differences in self-reported subjective well-being measures (i.e., Social-Emotional Health Survey-Secondary [SEHS-S]) across students based on the amount of school they reported missing in the past 30 days (i.e., Question 19)?

\section{Analysis Results}

Mean scores from the SEHS-S are provided in Table 4 in groups based on student responses to Question 19. An apparent decrease was evident in the subjective well-being of students who reported missing more school in the past 30 days. When converted to Average Item Response (AIR) scores (Furlong et al., 2020), SEHS-S results for students who reported missing 3 or more days of school remained in the average range. However, these students' scores were clearly lower than students who missed less school and demonstrated a growing standard deviation in their scores. The nonparametric K-W test analyzed scores on the SEHS-S based on the amount of absenteeism reported on Question 21 to determine if a significant difference existed between scores. The following nonparametric tests relied on median score comparisons; however, Table 4 presented mean scores to indicate the change in overall scores for each response to Question 19.

\section{Table 4}

SEHS-S Mean Scores for Question 19 Responses

\begin{tabular}{rrcc}
\hline $\begin{array}{l}\text { Question 19: In the past 30 days, how often did you } \\
\text { miss an entire day of school for any reason? }\end{array}$ & $n$ & $\begin{array}{c}\text { Mean SEHS-S } \\
\text { Scores }\end{array}$ & $\begin{array}{c}\text { Std. } \\
\text { Deviation }\end{array}$ \\
\hline 0 days & 45509 & 108.08 & 20.60 \\
1 day & 21669 & 106.47 & 20.55 \\
2 days & 14155 & 104.32 & 20.71 \\
3 or more days & 12428 & 100.72 & 22.03 \\
\hline
\end{tabular}


Results from the K-W test indicated a statistically significant difference in measures of subjective well-being (SEHS-S) across the four responses to Question 19, such that $\chi^{2}(3, n=$ $93,761)=1,261.34, p=.000$ (see Table 4 above for frequency distributions across each group level). Pairwise comparisons demonstrated in Table 5 revealed that SEHS-S scores are significantly different across each response to Question 19 and decrease as students missed more school. Additionally, effect size calculations were calculated to establish which group comparisons demonstrated significance and to what extent. Effect sizes for each group comparison further established a growing difference in SEHS-S scores as students reported missing more school. Using Glass' $\Delta$, the magnitude of difference in SEHS-S scores between students who reported missing 0 days compared to 3 or more days of school was between a small and medium level of effect (Glass' $\Delta=.36$ ).

\section{Table 5}

Pairwise Comparisons of SEHS-S Scores from Question 19 Responses

\begin{tabular}{|c|c|c|c|c|c|}
\hline Question 19 & Test Statistic & Std. Error & $\begin{array}{l}\text { Std. Test } \\
\text { Statistic }\end{array}$ & Adj. Sig. ${ }^{a}$ & $\begin{array}{c}\text { Glass' } \Delta \\
\text { Effect Size }\end{array}$ \\
\hline $\begin{array}{l}0 \text { days- } \\
1 \text { day }\end{array}$ & 2113.579 & 223.374 & 9.462 & .000 & .08 \\
\hline $\begin{array}{l}0 \text { days- } \\
2 \text { days }\end{array}$ & 5012.219 & 260.460 & 19.244 & .000 & .18 \\
\hline $\begin{array}{l}0 \text { days- } \\
3 \text { or more days }\end{array}$ & 9134.599 & 273.915 & 33.348 & .000 & .36 \\
\hline $\begin{array}{l}1 \text { day- } \\
2 \text { days }\end{array}$ & 2898.640 & 292.483 & 9.910 & .000 & .11 \\
\hline $\begin{array}{l}1 \text { day- } \\
3 \text { or more days }\end{array}$ & 7021.021 & 304.527 & 23.055 & .000 & .28 \\
\hline $\begin{array}{l}2 \text { days- } \\
3 \text { or more days }\end{array}$ & 4122.381 & 332.686 & 12.391 & .000 & .17 \\
\hline
\end{tabular}


Note. Each row tests the null hypothesis that the Sample 1 and Sample 2 distributions are the same; the significance level is .050 .

a. Significance values have been adjusted by the Bonferroni correction for multiple tests

Research Question 3b: Are there differences in self-reported subjective well-being measures (i.e., SEHS-S) across students based on the amount of school they reported skipping or class they were cutting in the past 12 months (Question 21)?

\section{Analysis Results}

By comparing mean scores from the SEHS-S across student responses to Question 21, Table 6 presented a similar trend of decreased subjective well-being for students who reported skipping or cutting school more frequently in the past 12 months. When converted to Average Item Response (AIR) scores (Furlong et al., 2020), SEHS-S results for students who reported skipping or cutting school more than once a week remained in the average range. However, a trend of decreased subjective well-being presented in students who reported skipping or cutting more school. The nonparametric alternative K-W test was conducted to determine if there were significant differences between students' scores on the SEHS-S based on the amount of school they reported skipping for Question 21. Although the nonparametric tests used median scores for the SEHS-S, Table 6 provided mean scores to indicate the difference in overall scores as students reported skipping more school.

\section{Table 6}

SEHS-S Mean Scores for Question 21 Responses

\begin{tabular}{lrrrr}
\hline $\begin{array}{l}\text { Question 21: During the past } 12 \text { months, about how } \\
\text { many times did you skip school or cut classes? }\end{array}$ & $n$ & Mean & Std. \\
\hline & 0 times & 70494 & SEHS-S Score & Deviation \\
\hline & $1-2$ times & 11403 & 107.90 & 20.49 \\
\end{tabular}




\begin{tabular}{rccc}
\hline $\begin{array}{l}\text { Question 21: During the past } 12 \text { months, about how } \\
\text { many times did you skip school or cut classes? }\end{array}$ & $n$ & $\begin{array}{c}\text { Mean } \\
\text { SEHS-S Score }\end{array}$ & $\begin{array}{c}\text { Std. } \\
\text { Deviation }\end{array}$ \\
\hline A few times & 7756 & 101.08 & 21.16 \\
Once a month & 840 & 99.58 & 21.89 \\
Twice a month & 1175 & 98.78 & 21.79 \\
Once a week & 798 & 97.09 & 21.03 \\
More than once a week & 1475 & 91.79 & 23.68 \\
\hline
\end{tabular}

Results from the K-W test indicated a statistically significant difference in measures of subjective well-being (SEHS-S) across the seven response options for Question 21 regarding the amount of school students skipped or cut, such that, $\chi^{2}(6, n=93,941)=2,174.15, p=.000$ (see Table 6 above for frequency distributions for each response). Pairwise comparisons presented in Table 7 revealed that SEHS-S scores were significantly different for a majority of responses to Question 21. No significant difference was found in SEHS-S scores during pairwise comparisons for students who reported skipping once a month and a few times ( $p=1.0)$; twice a month and once a month $(p=1.0)$; once a week and once a month $(p=.143)$; and once a week and twice a month $(p=1.0)$. These findings suggested that the difference occurring in students' subjective well-being reached a plateau after skipping school more than once a month.

Glass' $\Delta$ effect size calculations indicated the magnitude of effect regarding the difference between SEHS-S scores and each group from the pairwise comparisons and were included in Table 7. The most substantial difference was approaching a large effect size (.79) when comparing SEHS-S scores for students who reported skipping or cutting 0 times and those who reported more than once a week. Additional effect sizes between a small and medium level of effect occurred between students who reported skipping 0 times and once a month (.41), 0 times and twice a month (.45), and 0 times and once a week (.53), a few times, and more than once a week (.44), and 1-2 times and more than once a week (.52). 


\section{Table 7}

Pairwise Comparison of SEHS-S Scores from Question 21 Responses

\begin{tabular}{|c|c|c|c|c|c|}
\hline Question 21 & $\begin{array}{c}\text { Tests } \\
\text { Statistic }\end{array}$ & $\begin{array}{l}\text { Std. } \\
\text { Error }\end{array}$ & $\begin{array}{l}\text { Std. Test } \\
\text { Statistic }\end{array}$ & $\begin{array}{l}\text { Adj. } \\
\text { Sig. }^{a}\end{array}$ & $\begin{array}{r}\text { Glass' } \Delta \\
\text { Effect Size }\end{array}$ \\
\hline 0 times-1-2 times & 6779.521 & 273.696 & 24.770 & .000 & .25 \\
\hline 0 times- $A$ few times & 8801.493 & 324.390 & 27.132 & .000 & .33 \\
\hline 0 times-Once a month & 10213.342 & 941.138 & 10.852 & .000 & .41 \\
\hline 0 times-Twice a month & 11429.210 & 797.611 & 14.329 & .000 & .45 \\
\hline 0 times-Once a week & 13841.200 & 965.303 & 14.339 & .000 & .53 \\
\hline 0 times-More than once a week & 19035.368 & 713.381 & 26.683 & .000 & .79 \\
\hline 1-2 times- $A$ few times & 2021.972 & 399.097 & 3.066 & .000 & .08 \\
\hline 1-2 times-Once a month & 3433.821 & 969.428 & 3.542 & .008 & .15 \\
\hline 1-2 times-Twice a month & 4649.688 & 830.803 & 5.597 & .000 & .19 \\
\hline 1-2 times-Once a week & 7061.679 & 992.904 & 7.112 & .000 & .27 \\
\hline 1-2 times-More than once a week & 12255.847 & 750.308 & 16.334 & .000 & .52 \\
\hline A few times-Once a month & 1411.849 & 984.941 & 1.433 & 1.000 & .07 \\
\hline A few times-Twice a month- & 2627.717 & 848.854 & 3.096 & .041 & .11 \\
\hline A few times-Once a week & 5039.707 & 1008.057 & 4.999 & .000 & .19 \\
\hline A few times-More than once a week & 10233.876 & 770.247 & 13.286 & .000 & .44 \\
\hline Once a month-Twice a month & 1215.867 & 1225.179 & .992 & 1.000 & .04 \\
\hline Once a month-Once a week & 3627.858 & 1340.407 & 2.707 & .143 & .11 \\
\hline Once a month-More than once a week & 8822.026 & 1172.089 & 7.527 & .000 & .36 \\
\hline Once a week-Twice a month & 2411.991 & 1243.838 & 1.939 & 1.000 & .08 \\
\hline More than once a week-Twice a month & 7606.159 & 1060.300 & 7.174 & .000 & .32 \\
\hline More than once a week-Once a week & 5194.168 & 1191.579 & 4.359 & .000 & .25 \\
\hline
\end{tabular}

Note. Each row tests the null hypothesis that the Sample 1 and Sample 2 distributions are the same; the significance level is .050.

a. Significance values have been adjusted by the Bonferroni correction for multiple tests.

Research Question 4a: Are there differences in self-reported psychological distress measures (i.e., Social-Emotional Distress Scale-Secondary [SEDS-S]) across students based on the amount of school they reported missing in the past 30 days (i.e., Question 19)? 


\section{Analysis Results}

By comparing mean scores from the SEDS-S across student responses to Question 19, Table 8 indicated a noticeable increase in distress for students who reported missing more school in the past 30 days. When converted to Average Item Response (AIR) scores (Furlong et al., 2020), SEHS-S results for students who reported missing 3 or more days remained in the average range. However, a clear trend of increased psychological distress occurred in students who reported missing more school. The nonparametric alternative $\mathrm{K}-\mathrm{W}$ test was conducted to determine if there were significant differences between students' SEDS-S scores based on the amount of absences they reported on Question 19. While the following nonparametric tests relied on median score comparisons, Table 8 presented mean SEDS-S scores to indicate overall changes as students reported missing more school.

\section{Table 8}

SEDS-S Mean Scores for Question 19 Responses

\begin{tabular}{lrrrr}
\hline $\begin{array}{l}\text { Question 19: In the past 30 days, how often did you miss } \\
\text { an entire day of school for any reason? }\end{array}$ & $n$ & $\begin{array}{c}\text { Mean } \\
\text { SEDS-S Score }\end{array}$ & Std. Deviation \\
\hline 0 days & 49486 & 19.18 & 8.29 \\
1 day & 23563 & 20.09 & 8.57 \\
2 days & 15602 & 20.90 & 8.83 \\
3 or more days & 13808 & 22.05 & 9.27 \\
\hline
\end{tabular}

Results from the K-W test indicated a statistically significant difference in measures of psychological distress (SEDS-S) across the four response options for Question 19 regarding the amount of school students reported missing, such that, $\chi^{2}(3, n=102,459)=1,261.34, p=.000$ (see Table 8 above for frequency distributions for each response). Pairwise comparisons demonstrated in Table 9 revealed that SEDS-S scores were significantly different across each 
response as students reported increased absenteeism. Additionally, effect size calculations established which group comparisons demonstrated significance and to what extent and indicated a growing difference in SEDS-S scores as students reported missing more school. Using Glass' $\Delta$, the magnitude of difference between students who reported missing 0 days compared to 3 or more days of school was between a small and medium level of effect (Glass’ $\Delta=.35$ ).

\section{Table 9}

Pairwise Comparisons of SEDS-S Scores from Question 19 Responses

\begin{tabular}{|c|c|c|c|c|c|}
\hline Question 19 & Test Statistic & Std. Error & $\begin{array}{l}\text { Std. Test } \\
\text { Statistic }\end{array}$ & Adj. Sig. ${ }^{a}$ & $\begin{array}{c}\text { Glass' } \Delta \text { Effect } \\
\text { Size }\end{array}$ \\
\hline $\begin{array}{l}0 \text { days- } \\
1 \text { day }\end{array}$ & -3144.766 & 233.60 & -13.46 & .000 & .11 \\
\hline $\begin{array}{l}0 \text { days- } \\
2 \text { days }\end{array}$ & -5760.074 & 270.98 & -21.26 & .000 & .21 \\
\hline $\begin{array}{l}0 \text { days- } \\
3 \text { or more days }\end{array}$ & -9114.926 & 284.05 & -32.09 & .000 & .35 \\
\hline $\begin{array}{l}1 \text { day- } \\
2 \text { days }\end{array}$ & -2615.307 & 304.62 & -8.59 & .000 & .09 \\
\hline $\begin{array}{l}1 \text { day- } \\
3 \text { or more days }\end{array}$ & -5970.159 & 316.31 & -18.88 & .000 & .23 \\
\hline $\begin{array}{l}2 \text { days- } \\
3 \text { or more days }\end{array}$ & -3354.852 & 344.84 & -9.73 & .000 & .13 \\
\hline
\end{tabular}

Note. Each row tests the null hypothesis that the Sample 1 and Sample 2 distributions are the same; the significance level is .050.

a. Significance values have been adjusted by the Bonferroni correction for multiple tests.

Research Question 4b: Are there differences in self-reported psychological distress measures (i.e., SEDS-S) across students based on the amount of school they reported skipping or class they were cutting in the past 12 months (Question 21)? 


\section{Analysis Results}

By comparing mean scores from the SEDS-S across student responses to Question 21, Table 10 indicated a similar trend of increased psychological distress for students who reported skipping or cutting more school in the past 12 months. When converted to Average Item Response (AIR) scores (Furlong et al., 2020), SEDS-S results for students who reported skipping or cutting school more than once a week remained in the average range. However, similar to previous results, a clear trend of increased psychological distress was observed in students who reported skipping or cutting more school. The nonparametric alternative K-W test was conducted to determine if there were significant differences between students' SEDS-S scores based on the amount of absenteeism they reported on Question 21. The following nonparametric tests relied on median score comparisons; however, Table 10 presented mean scores to indicate the overall change occurring as students reported skipping more school.

\section{Table 10}

SEDS-S Mean Scores for Question 21 Responses

\begin{tabular}{|c|c|c|c|}
\hline $\begin{array}{l}\text { Question 21: During the past } 12 \text { months, about how many } \\
\text { times did you skip school or cut classes? }\end{array}$ & $n$ & $\begin{array}{l}\text { Mean SEHS-S } \\
\text { Score }\end{array}$ & $\begin{array}{c}\text { Std. } \\
\text { Deviation }\end{array}$ \\
\hline 0 times & 76969 & 19.51 & 8.48 \\
\hline 1-2 times & 12470 & 21.02 & 8.68 \\
\hline A few times & 8504 & 21.73 & 8.83 \\
\hline Once a month & 944 & 22.45 & 9.30 \\
\hline Twice a month & 1279 & 22.99 & 9.19 \\
\hline Once a week & 860 & 23.07 & 9.34 \\
\hline More than once a week & 1636 & 23.21 & 9.74 \\
\hline
\end{tabular}

Results from the K-W test indicated a statistically significant difference in measures of psychological distress (SEDS-S) across the seven response options for Question 21 regarding the 
amount of school skipped or classes cut, such that $\chi^{2}(6, n=102,662)=1,276.89, p=.000$ (see Table 10 above for frequency distributions of each response). Pairwise comparisons demonstrated in Table 11 revealed significantly different SEDS-S scores as responses indicated more frequent absenteeism. However, reports of progressively increasing psychological distress ceased when students reported skipping or cutting school once a month or more. A significant difference was not found when comparing SEDS-S scores from students who reported skipping or cutting once a month and a few times $(p=1.0)$ and each subsequent comparison of responses: once a month and more (once a month and twice a month $[p=1.0]$; once a month and once a week $[p=1.0]$; once a month and more than once a week $[p=1.0]$; twice a month and once a week $[p=.984]$; more than once a week and twice a month $[p=1.0]$; and more than once a week and once a week $[p=.775]$. These findings seem to support previous outcomes that demonstrated a plateau in measures of student mental health after they reported skipping or cutting school more than once a month.

Glass' $\Delta$ was used to calculate effect sizes, presented in Table 11, to indicate the magnitude of effect for SEDS-S scores between each response from Question 21. When comparing students who reported skipping or cutting the least amount ( 0 times) to the highest amount (more than once a week) of school, the most substantial effect size (.44) remained in the small effect size range. Additionally, effect sizes were between a small and medium level of effect when comparing students who reported skipping 0 times and once a month (.35), 0 times and twice a month (.41), and 0 times and once a week (.42). 


\section{Table 11}

Pairwise Comparisons of SEDS-S Scores from Question 21 Responses

\begin{tabular}{|c|c|c|c|c|c|}
\hline Question 21 & Test Statistic & $\begin{array}{l}\text { Std. } \\
\text { Error }\end{array}$ & $\begin{array}{l}\text { Std. Test } \\
\text { Statistic }\end{array}$ & $\begin{array}{l}\text { Adj. } \\
\text { Sig. }^{\text {a }}\end{array}$ & $\begin{array}{c}\text { Glass' } \Delta \\
\text { Effect Size }\end{array}$ \\
\hline 0 times- $1-2$ times & -5461.180 & 285.461 & -19.131 & .000 & .18 \\
\hline 0 times- $A$ few times & -7754.630 & 337.924 & -22.948 & .000 & .26 \\
\hline 0 times- Once a month & -9531.119 & 968.357 & -9.843 & .000 & .35 \\
\hline 0 times- Twice a month & -11456.439 & 833.716 & -13.741 & .000 & .41 \\
\hline 0 times- Once a week & -11481.875 & 1014.000 & -11.323 & .000 & .42 \\
\hline 0 times- More than once a week & -11125.118 & 738.839 & -15.058 & .000 & .44 \\
\hline 1-2 times- $A$ few times & -2293.451 & 415.882 & -5.515 & .000 & .08 \\
\hline 1-2 times- Once a month & -4069.939 & 998.239 & -4.077 & .001 & .16 \\
\hline 1-2 times- Twice a month & -5995.259 & 868.244 & -6.905 & .000 & .23 \\
\hline 1-2 times-Once a week & -6020.695 & 1042.575 & -5.775 & .000 & .24 \\
\hline 1-2 times-More than once a week & -5663.938 & 777.592 & -7.284 & .000 & .25 \\
\hline A few times- Once a month & -1776.488 & 1014.488 & -1.751 & 1.00 & .08 \\
\hline A few times- Twice a month & -33701.809 & 886.878 & -4.174 & .001 & .14 \\
\hline A few times-Once a week & -3727.245 & 1058.143 & -3.522 & .009 & .15 \\
\hline A few times-More than once a week & -3370.487 & 798.344 & -4.222 & .001 & .17 \\
\hline Once a month- Twice a month & -1925.321 & 1268.887 & -1.517 & 1.00 & .06 \\
\hline Once a month-Once a week & -1950.757 & 1393.983 & -1.399 & 1.00 & .07 \\
\hline Once a month-More than once a week & -1593.999 & 1208.667 & -1.319 & 1.00 & .08 \\
\hline Once a week-Twice a month & -25.436 & 1304.054 & -.020 & 1.00 & .01 \\
\hline More than once a week- Twice a month & 331.321 & 1103.740 & .300 & 1.00 & .02 \\
\hline More than once a week- Once a week & 356.757 & 1245.535 & .286 & 1.00 & .01 \\
\hline
\end{tabular}

Note. Each row tests the null hypothesis that the Sample 1 and Sample 2 distributions are the same; the significance level is .050 .

a. Significance values have been adjusted by the Bonferroni Correction for multiple tests.

Research Question 5: Using groups (a) Complete Mental Health, (b) Symptomatic but Content,

(c) Vulnerable, and (d) Troubled from a dual-factor model approach, what percentage of students from each group presented with potential SRB? 


\section{Analysis Results}

Using the dual-factor model $(n=26,957)$, students were broken into groups of Complete Mental Health (average to high subjective well-being and low psychopathology $[n=10,540$; 39.1\%]), Symptomatic but Content (average to high subjective well-being and high psychopathology $[n=12,014 ; 44.6 \%]$ ), Vulnerable (low subjective well-being and low psychopathology $[n=1,699 ; 6.3 \%]$ ), and Troubled (low subjective well-being and high psychopathology $[2,704 ; 10.0 \%])$.

Cross-tabulations tables, presented in Table 12, provided frequency distributions for each dual-factor group and the responses from Question 19 regarding attendance. Results indicated that $23.7 \%$ of students in the Troubled group $(n=2,697)$ and $22.1 \%$ from the Vulnerable group $(n=1,675)$ reported missing 3 or more days of school in the past 30 days. Comparatively, this was more than double the percentage of students from the Complete Mental Health group who reported missing 3 or more days of school $(n=10,479 ; 10.1 \%)$. Additionally, fewer students from the Troubled group reported missing 0 days of school than the other dual-factor groups (Complete Mental Health, Symptomatic but Content, and Vulnerable). A Chi-square test for independence analyzed the relationship between attendance and the dual-factor groups. Results from this analysis indicated a significant association between attendance and the dual-factor groups using Question 19, $\chi^{2}(9, n=26,840)=777.24, p=<.001$, Cramer's $V=.098$.

\section{Table 12}

Dual-Factor Group Distributions for Question 19 Responses

\begin{tabular}{lcccccc}
\hline Dual Factor Groups & & 0 days & 1 day & 2 days & or more days & Total \\
\hline \multirow{2}{*}{ Complete Mental Health } & $n$ & 5715 & 2289 & 1420 & 1055 & 10479 \\
& $(\%)$ & $(52.5 \%)$ & $(21.8 \%)$ & $(13.6 \%)$ & $(10.1 \%)$ & $(100.0 \%)$ \\
Symptomatic but Content & $n$ & 4931 & 2779 & 2127 & 2152 & 11989
\end{tabular}




\begin{tabular}{lcccccc}
\hline Dual Factor Groups & & 0 days & 1 day & 2 days & 3 or more days & Total \\
\hline \multirow{3}{*}{ Vulnerable } & $(\%)$ & $(41.1 \%)$ & $(23.2 \%)$ & $(17.7 \%)$ & $(17.9 \%)$ & $(100.0 \%)$ \\
& $n$ & 685 & 341 & 278 & 371 & 1675 \\
Troubled & $(\%)$ & $(40.9 \%)$ & $(20.4 \%)$ & $(16.6 \%)$ & $(22.1 \%)$ & $(100.0 \%)$ \\
& $n$ & 974 & 598 & 485 & 640 & 2697 \\
Total & $(\%)$ & $(36.1 \%)$ & $(22.2 \%)$ & $(18.0 \%)$ & $(23.7 \%)$ & $(100.0 \%)$ \\
& $n$ & 12305 & 6007 & 4310 & 4218 & 26840 \\
& $(\%)$ & $(45.8 \%)$ & $(22.4 \%)$ & $(16.1 \%)$ & $(15.7 \%)$ & $(100.0 \%)$ \\
\hline
\end{tabular}

Similarly, Table 13 provided cross-tabulation tables for each dual-factor group and responses to Question 21 regarding attendance. Consistent with previous results, higher percentages of Troubled youth reported skipping school. Representation from the Troubled group was over five times greater than the percentage of students from the Complete Mental Health group in reports of skipping school more than once a week. The Vulnerable group persisted with results similar to Troubled students, presenting an even higher percentage of students skipping more than once a week. A Chi-square test for independence analyzed the relationship between attendance and the dual-factor groups. Results from this analysis indicated a significant association between attendance and the dual-factor groups based on Question 21, $\chi^{2}$ $(18, n=26,896)=1018.508, p=<.001$, Cramer's $V=.112$.

\section{Table 13}

Dual-Factor Group Distributions for Question 21 Responses

\begin{tabular}{|c|c|c|c|c|c|c|c|c|c|}
\hline \multicolumn{2}{|c|}{ Dual Factor Groups } & \multirow{2}{*}{$\begin{array}{c}0 \text { times } \\
8634\end{array}$} & \multirow{2}{*}{$\begin{array}{c}\text { 1-2 } \\
\text { times } \\
984\end{array}$} & \multirow{2}{*}{$\begin{array}{c}\text { A few } \\
\text { times }\end{array}$} & \multirow{2}{*}{$\begin{array}{c}\begin{array}{c}\text { Once a } \\
\text { month }\end{array} \\
61\end{array}$} & \multirow{2}{*}{$\begin{array}{c}\begin{array}{c}\text { Twice a } \\
\text { month }\end{array} \\
84\end{array}$} & \multirow{2}{*}{$\begin{array}{c}\begin{array}{c}\text { Once a } \\
\text { week }\end{array} \\
51\end{array}$} & \multirow{2}{*}{$\begin{array}{c}\text { More than } \\
\text { once a week }\end{array}$} & \multirow{2}{*}{$\begin{array}{c}\text { Total } \\
10510\end{array}$} \\
\hline Complete Mental & $n$ & & & & & & & & \\
\hline Health & $(\%)$ & $(82.2 \%)$ & $(9.4 \%)$ & $(5.6 \%)$ & $(0.6 \%)$ & $(0.8 \%)$ & $(0.5 \%)$ & $1.0 \%)$ & $(100.0 \%)$ \\
\hline Symptomatic but & $n$ & 8386 & 1601 & 1217 & 150 & 215 & 156 & 274 & 11999 \\
\hline Content & $(\%)$ & $(69.9 \%)$ & $(13.3 \%)$ & $(10.1 \%)$ & $(1.3 \%)$ & $(1.8 \%)$ & $(1.3 \%)$ & $(2.3 \%)$ & $(100.0 \%)$ \\
\hline \multirow{2}{*}{ Vulnerable } & $n$ & 1084 & 234 & 187 & 30 & 43 & 21 & 91 & 1690 \\
\hline & $(\%)$ & $(64.1 \%)$ & $(13.8 \%)$ & $(11.1 \%)$ & $(1.8 \%)$ & $(2.5 \%)$ & $(1.2 \%)$ & $(5.4 \%)$ & $(100.0 \%)$ \\
\hline
\end{tabular}




\begin{tabular}{|c|c|c|c|c|c|c|c|c|c|}
\hline \multicolumn{2}{|c|}{ Dual Factor Groups } & \multirow{2}{*}{$\begin{array}{c}0 \text { times } \\
1573\end{array}$} & \multirow{2}{*}{$\begin{array}{c}\text { 1-2 } \\
\text { times } \\
440\end{array}$} & \multirow{2}{*}{$\begin{array}{l}\text { Afew } \\
\text { times } \\
371\end{array}$} & \multirow{2}{*}{$\begin{array}{c}\text { Once a } \\
\text { month } \\
50\end{array}$} & \multirow{2}{*}{$\begin{array}{c}\text { Twice a } \\
\text { month } \\
81\end{array}$} & \multirow{2}{*}{$\begin{array}{c}\text { Once a } \\
\text { week } \\
45\end{array}$} & \multirow{2}{*}{$\begin{array}{c}\text { More than } \\
\text { once a week } \\
137\end{array}$} & \multirow{2}{*}{$\begin{array}{l}\text { Total } \\
2697\end{array}$} \\
\hline & $n$ & & & & & & & & \\
\hline I roubled & $(\%)$ & $(58.3 \%)$ & $(16.3 \%)$ & $(13.8 \%)$ & $(1.9 \%)$ & $(3.0 \%)$ & $(1.7 \%)$ & $(5.1 \%)$ & $(100.0 \%)$ \\
\hline \multirow{2}{*}{ Total } & $n$ & 19677 & 3259 & 2364 & 291 & 423 & 2273 & 609 & 26896 \\
\hline & $(\%)$ & $(73.2 \%)$ & $(12.1 \%)$ & $(8.8 \%)$ & $(1.1 \%)$ & $(1.6 \%)$ & $(1.0 \%)$ & $(2.3 \%)$ & $(100.0 \%)$ \\
\hline
\end{tabular}




\section{Chapter 5: Discussion}

Analysis results will be provided for each research question in the following sections. Findings for each research question included a discussion of its implication and relevance to other research. Following these results, a summary of strengths, weaknesses, and overall limitations summarized the present study. Finally, an overview of the present study and its implications for practice and future research provided direction to scholars and practitioners. The present study corroborated findings from previous research to identify gaps in the literature regarding the mental health of students with SRB. Little to no research has considered a dualfactor approach to analyzing changes to mental health as students miss or skip school. Future research is strongly encouraged to continue pursuing a better understanding of these students, their mental health, and how to best support them.

Research Question 1: How do school attendance items from the current dataset compare to prevalence outcomes using other large datasets?

\section{Research Question 1 Findings}

Establishing an accurate prevalence of SRB has faced a longstanding plight of inconsistent methods for collecting, identifying, and measuring absenteeism. Students categorized as truant are often at the focal point of questions about attendance that typically inquire about skipping or cutting school. The CHKS-SCM provided some reprieve from this by using Question 19 (missed school) in addition to Question 21 (skipped or cut school). Each question represented different functions of absenteeism. However, this distinction remains unclear. While $48 \%$ of students from Question 19 reported having missed 0 days of school in the past 30 days, $74.7 \%$ of students from Question 21 reported having skipped or cut 0 times in the 
past 12 months. The present study sought to accommodate these differences by utilizing both questions in its analyses.

As previously mentioned, Kearney (2008) suggested that outcomes can become notably problematic for students who missed 2.5 days of school in a given two-week period (i.e., $25 \%$ ) or were absent for ten days over 15 weeks (i.e., 13\%). The present study focused on groups of students who reported missing 3 or more days in the past 30 days or skipping twice a month or more in the past 12 months. Each of these groups represented students who, at the very least, present a high risk for SRB and its subsequent negative impact. Earlier thresholds for identifying SRB struggles recommend intervening for students after $10 \%$ of school was missed over three months (Lyon \& Cotler, 2007).

From Question $19(n=106,651), 13.5 \%$ of students responded to having missed 3 or more days of school in the past 30 days. These students posed a risk of missing approximately $15 \%$ of the available school days. On the other hand, Responses from Question $21(n=106,857)$ indicated that $3.6 \%$ of students reported skipping or cutting twice a month $(1.2 \%)$, once a week $(0.8 \%)$, or more than once a week $(1.6 \%)$, combined, in the 12 months prior. In California, each school year consists of 180 school days (National Center for Education Statistics, 2021). This range (twice a month through more than once a week) included the potential of skipping approximately $11 \%$ or more $(>40 \%)$ of available school- assuming that students skipped school for the entire day.

The CHKS-SCM is accessible and specific to California schools, making it a valuable tool for gathering and analyzing attendance information. Previous literature has frequently cited a broad range from 5-28\% for students presenting with SRB (Heyne et al., 2019; Kearney, 2001; Lyon \& Cotler, 2007). The caution maintained in this broad range was likely intended to avoid 
excluding students at-risk for SRB and emphasized the growing issue of SRB. While the CHKSSCM attendance questions resulted in similar outcomes, a more narrow prevalence of SRB (3.6$13.5 \%$ ) was found when compared.

On the other hand, compared to other large survey datasets, the present study resulted in a higher percentage of student absences. Data from the 2009 National Survey on Drug Use and Health $(n=17,482)$ found $11 \%$ of students ages $12-17$ years old reported skipping school in the past 30 days (Vaughn et al., 2013). Findings from the 2007 School Crime Survey report $(n=$ 5,621 ) found that only 5.5\% of students ages $12-18$ reported skipping school in the month before the survey (United States Department of Justice, 2007). And other large surveys (Monitoring the Future, $n=11,113$ [Henry, 2007]; National Comorbidity Survey-Adolescent Supplement, $n=$ 9,244 [Kessler, 2001-2004]) used similar language for students to report the amount of school they had skipped and found 11-27\% of adolescents reporting to have skipped school.

Unfortunately, incongruent terms and definitions for SRB continue to deter effective classification and understanding of these students. The studies mentioned above, for instance, relied on questions targeting students through truancy courts or surveys of mental health, school crime, and drug use. When approaching attendance from this position, a subsequent impact may occur when attempting to understand attendance patterns. The CHKS-SCM provided some relief from this and allowed school-based data to be considered. While obstacles to gaining accurate prevalence information for SRB persist, these components support understanding how many students have attendance problems and why.

Research Question 2a: Are significant differences observed in attendance groups based on grade level? 


\section{Research Question 2a Findings}

Regarding Question 19, 11.7\% of students in grade 7 reported missing 3 or more days of school in the past 30 days compared to $17.9 \%$ of students in grade 12 . More students reported missing 3 or more days of school more as they aged through grades 7 (11.7\%), 8 (12.5\%), 9 $(12.6 \%), 10(13.8 \%), 11(15.2 \%)$, and $12(17.9 \%)$. A similar trend existed in responses to Question 21 with increased collective responses for students who skipped school twice a month or more (twice a month, once a week, and more than once a week) in grades $7(1.6 \%), 8(1.8 \%)$, $9(2.9 \%), 10(3.9 \%), 11(5.7 \%)$, and $12(8.2 \%)$. An analysis of these results found a statistically significant association between attendance and grade level with an increase of absenteeism in older students; the effect sizes from these outcomes were small.

Previous literature has reported that SRB occurred most commonly between ages 10-13 years old and during transitions from elementary to middle or middle to high school settings (Kearney \& Albano, 2018; Kearney et al., 2004). Other findings, however, have indicated consistently higher rates of absenteeism as students grow older (Maynard et al., 2017). The present study relied on children aged 12-17 years old, focusing on secondary-age students. Findings supported prior studies in which a progressive increase occurred in secondary students missing or skipping school more often as they grew older.

Research Question 2b: Is there a significant association between attendance and gender or sexual orientation?

\section{Research Question 2b Findings}

The present study found that higher rates of female students (15.1\%) reported missing 3 or more days of school in the past 30 days than males $(11.8 \%)$. These findings remained consistent for each response to Question 21, in which more female students reported skipping 
school in the past 12 months than male students. Female students continually represented more elevated rates of absence in secondary students. Consistent with previous studies, an even higher rate of transgender students (21.3\%) reported missing 3 or more days in the past 30 days. Analyses for the relationships between gender (male or female and transgender students, separately) and absenteeism yielded statistically significant results.

Further analysis of students' sexual orientation indicated that reports from gay or lesbian and bisexual students (18.4\%) who missed 3 or more days of school in the past 30 days were more likely than straight (not gay) students and those who declined to respond (12.9\%). Similar results from Question 21 supported that gay or lesbian and bisexual students reported skipping school more often in the past 12 months. The relationship between sexual orientation and attendance reports from Questions 19 and 21 were also statistically significant. Students' gender identity and sexual orientation appeared to influence attendance and pose critical implications for students who face a higher risk of SRB.

Previous literature has identified different truancy rates for male and female students, such that females present more frequent absences. Although, due to the persistent neglect for consistently defining "truancy" behaviors, outcomes have varied. Certain studies have reported more likely absences for males due to truancy behaviors (Maynard et al., 2017). Other studies have maintained that gender may not play a significant role in attendance (Henry, 2007; Kearney et al., 2004; Vaughn et al., 2013). When considering transgender students, a nascent body of literature has found, among other things, a significantly higher likelihood of missing more than three days of school out of the past 30 days and higher risks for unexcused absenteeism (Pampati et al., 2020; Robinson \& Espelage, 2011). 
Research Question 2c: Are significant differences observed in attendance groups based on student race/ethnicity?

\section{Research Question 2c Findings}

School attendance problems pose a growing concern across all race/ethnicity groups. However, not unlike many issues in education, these concerns are not proportionate across all minority groups of people. The present study found that Hispanic/Latinx students reported missing and skipping school more often than non-Hispanic/Latinx students. This margin of difference remained small across each response option for Questions 19 and 21; however, it presented a statistically significant relationship. When considering additional race/ethnicities, a clear trend was present in Asian students consistently reporting to miss and skip school less than all other backgrounds, followed by Caucasian/White students.

Black or African American students reported higher rates of missing and skipping schoolalthough these rates were at times identical to American Indian or Alaska Native students and closely followed by Native Hawaiian or Pacific Islander and students of Mixed (two or more) races. Students' race/ethnicity had a statistically significant relationship with attendance based on Questions 19 and 21. Despite effect sizes for this analysis remaining small, when compared to the range of effect size outcomes for other demographic items (Cramer's $V=.022-.089$ ), race/ethnicity demonstrated the largest relative effect (.089) for its relationship with attendance using Question 19 (missed school over the past 30 days).

Findings from the present study were consistent with previous research outcomes. For instance, Vaughn and colleagues (2013) compared groups of mild, moderate, and chronic SRB across students' race/ethnicity. This study found that Caucasian/White students represented a majority of mild SRB cases (80\%), while African American and Hispanic students comprised a 
majority of moderate cases (approximately $80 \%$ ); chronic cases were more evenly allocated across these three groups. Additionally, studies of secondary students (12-17 years of age) found consistent disproportionalities with higher rates of absence for Hispanic and African American students when compared to Caucasian/White students (Maynard et al., 2017).

Research Question 2d: Is there a significant association between attendance and socioeconomic status?

\section{Research Question 2d Findings}

Socioeconomic background and attendance were analyzed using student reports of their parents' education levels and student eligibility for free or reduced-price lunch. Responding don't $k n o w$ to the question regarding parental education level excluded students from the analysis. Results indicated that fewer students whose parents had higher education levels reported missing 3 or more days of school in the past 30 days. This trend remained consistent when reviewing outcomes for Question 21 for reports on skipping school in the past 12 months. A statistically significant relationship was found between parental education level and attendance reports from Questions 19 and 21.

Further prospects were explored by analyzing students eligible for free or reduced-price lunch (self-reported). These results indicated that more students who were eligible for free or reduced-price lunch reported missing and skipping school than students who did not report being eligible. A statistically significant relationship between these variables was found to support the interaction between students' attendance and their socioeconomic background; effect size measures were small for this outcome.

Present findings were similar to other research outcomes in which parents with higher income correlated with better school attendance (Gennetian et al., 2018; Maynard et al., 2017). 
Findings from other studies have also asserted that students from low-income families and communities are four times likely to miss $10 \%$ or more of school when compared to peers from higher income groups (Chang et al., 2008; Gottfried, 2014). While SRB presents in all socioeconomic groups, its presence is not proportionate.

\section{Research Questions 2a-2d Findings Summary}

The present study sought to understand and acknowledge demographic factors and their undeniable role in SRB cases. Results found that grade level, gender, sexual orientation, race/ethnicity, and socioeconomic status have a significant relationship with attendance. Despite the effect sizes for each of these analyses remaining small, their outcomes support evidence from previous literature findings that emphasize demographic factors and their critical role in school attendance. These results warranted future research to develop and understand the different "risk profiles" associated with select demographic features in students with SRB (Maynard et al., 2017). School attendance problems present a growing social justice concern that afflicts potentially vulnerable people groups to a greater degree.

An analysis of the demographics from the CHKS-SCM sample resulted in outcomes similar to other studies. Specifically, students reported missing and skipping school more often as they grew older. More female students reported absenteeism than males, although transgender students represented absenteeism the most. More gay or lesbian and bisexual students reported missing and skipping school than their cisgender male or straight peers. More students of color were absent than White/Caucasian and Asian students. And children from families with less parental education and who were eligible for free or reduced-price lunch were absent more often. Unfortunately, these findings consistently confirm a trend in research outcomes that imply a student's demographic background significantly influences their school attendance. 
Research Questions 3a: Are there differences in self-reported subjective well-being measures (i.e., Social-Emotional Health Survey-Secondary [SEHS-S]) across students based on the amount of school they reported missing in the past 30 days (i.e., Question 19)?

\section{Research Questions 3a Findings}

The present study used a dual-factor approach to consider subjective well-being in addition to psychological distress when analyzing the interaction between attendance and a dualfactor perspective on mental health. The SEHS-S was relied on as an established measure of covitality- a synergistic view of subjective well-being. This model is built on factors of belief-inself, belief-in-others, engaged living, and emotional competence. By comparing the difference in overall SEHS-S scores across student responses to the CHKS-SCM questions on attendance, results indicated that overall subjective well-being progressively decreased in students who reported missing more school.

Overall, the mean SEHS-S scores remained within the average range for students who reported missing 3 or more days of school in the past 30 days. These findings implied that select students at-risk for SRB presented with average ratings of their subjective well-being. However, there was an apparent decrease in these scores with increased reports of missing school. After analyzing differences in SEHS-S scores based on student responses to Question 19, significant differences were present between each response ( 0 days, 1 day, 2 days, and 3 or more days). A significant decrease was witnessed in the subjective well-being of students as they reported missing more school.

Even when comparing SEHS-S scores for students who missed 0 days and reports of missing 1 day, neither of which propose a risk of SRB, a significant difference remained; although, a small effect size was present (.08). For students who reported missing school more 
often, this significance became more substantial and rendered a small effect size (.36) when comparing students who reported missing 0 days and 3 or more days. Students who reported missing 3 or more days in the past 30 days posed a risk for SRB by missing $15 \%$ of the available school days. The present study found that the subjective well-being of these students was significantly lower than students who reported missing less school.

Previous research has scarcely studied SRB using a dual-factor approach to mental health; consequentially, literature provides little understanding of how attendance interacts with subjective well-being. However, a recent study used the SEHS-S to identify factors of subjective well-being, namely belief-in-self and belief-in-others, as significant indicators of attendance problems. The present study was unique in its approach for considering a dual-factor perspective on mental health. Findings support subjective well-being as having a critical role in students' attendance.

Research Question 3b: Are there differences in self-reported subjective well-being measures (i.e., SEHS-S) across students based on the amount of school they reported skipping or cutting in the past 12 months (i.e., Question 21)?

\section{Research Question 3b Findings}

To further inspect the interaction between subjective well-being and SRB, Question 21 was used to identify how much school students reported skipping in the past 12 months. As previously discussed, this question's language elicits a different meaning than Question 19 (missing school). This population of students, akin to the term truancy, can often be dismissed as maladjusted or merely misbehaving. However, research has identified underlying psychopathologies (e.g., depression or anxiety) as a common addition to student conduct problems or oppositional defiance in cases of SRB (Bools et al., 1990; Egger et al., 2003). 
Including the subjective well-being of these students was an essential step towards better understanding their mental health.

When comparing SEHS-S scores across responses to Question 21, a statistically significant difference was found. As reports of skipping school increased, subjective well-being decreased. Further comparison of each response to Question 21 indicated a significant difference between students who reported skipping 0 times and 1-2 times and 1-2 times and a few times. However, there was no statistical significance for differences in SEHS-S scores between students who reported skipping a few times and once a month; once a month and twice a month; once a month and once a week; as well as twice a month and once a week. These outcomes had important implications as the difference in SEHS-S scores appeared to cease in students who reported skipping school once a month and more.

Once students began skipping school once a month or more, the differences between scores became less dramatic. Even the difference between students who reported skipping a few times and once a month $(p=1.0)$ or a few times and twice a month $(p=.041)$ presented little-tono significance in the differences based on their SEHS-S scores. A statistically significant difference in SEHS-S scores resumed when comparing student reports of skipping once a week and more than once a week $(p=.000)$. Overall, there was a clear and significant difference in SEHS-S scores between students who reported skipping school 0 times or 1-2 times and each higher absenteeism report. Most notably, the magnitude of difference in SEHS-S scores between students who reported skipping 0 times and skipping more than once a week yielded a large effect size (.79). These findings indicated a drastic decrease in students' subjective well-being as they reported skipping school more often. 
Research Question 4a: Are there differences in self-reported psychological distress measures (i.e., Social-Emotional Distress Scale-Secondary [SEDS-S]) across students based on the amount of school they reported missing in the past 30 days (i.e., Question 19)?

\section{Research Question 4a Findings}

This study emphasized a dual-factor approach to mental health by analyzing students' psychological distress as a counterpart to their subjective well-being. To better understand the interaction between mental health and attendance, SEDS-S scores were compared across responses to Question 19 regarding attendance. Like results from the SEHS-S, after converting SEDS-S ratings into overall AIR scores, they remained in the average range across all responses to Question 19- even the group of students who reported missing 3 or more days in the past 30 days. However, after analyzing SEDS-S scores, a statistically significant difference indicated increased psychological distress in students as they reported missing more school.

After only one absence, compared to students who reported missing 0 days, a statistically significant difference existed between scores on the SEDS-S- although the effect size for this group comparison was small (.11). When analyzing students with a low risk of SRB (missed 0 days) and students who reported missing 3 or more days of school in the past 30 days, a small level of effect (.35) indicated a growing magnitude of difference in SEDS-S scores. This effect size was nearly identical to the same comparison when analyzing the SEHS-S (.36). Based on the overall comparison of subjective well-being and psychological distress, a significant change occurred in the mental health of students who reported more absenteeism.

While research on SRB has not strictly considered a dual-factor approach to mental, extensive literature has long recognized the strong connection with mental health. Research has consistently established a strong relationship between SRB and symptoms of anxiety and 
depression (Gonzálvez et al., 2019; Hansen et al., 1998; Honjo et al., 2001; Honjo et al., 2003;

Last, 1991). These symptoms often occur concurrently with underlying psychosomatic symptoms and have a strong correlation with absenteeism (Bernstein et al., 1997; Kearney \& Silverman,1993; McShane et al., 2004; Nayak et al., 2018). Additionally, students with anxietybased SRB difficulties are more subject to victimization and bullying (Delgado et al., 2019). Anxiety or depression has been found to manifest in several ways, including academic problems, peer rejection, and feeling inadequate- each of which can contribute to the severity and impact of SRB cases (Craun et al., 2017; McShane et al., 2004). Internalizing symptoms are also common underlying factors in SRB cases where primary concerns include externalizing behavior symptoms (Bools et al., 1990; Egger et al., 2003).

Research Question 4b: Are there differences in self-reported psychological distress measures (i.e., SEDS-S) across students based on the amount of school they reported skipping or cutting in the past 12 months (i.e., Question 21)?

\section{Research Question 4b Findings}

To further inspect the difference in psychological distress for students with SRB, students were identified based on Question 21 regarding the amount of school they reported skipping in the past 12 months. Similar to previous findings in this study, SEDS-S scores were significantly different based on students' responses to Question 21. By comparing students who reported skipping school 0 times or 1-2 times in the past 12 months, SEDS-S scores were significantly higher in each proceeding response (skipping a few times, once a month, twice a month, once a week, or more than once a week). However, when comparing students who reported skipping $a$ few times and skipping once a month, their SEDS-S scores were no longer significantly different. Additionally, SEDS-S scores from students who skipped once a month compared to reports of 
skipping more frequently (twice a month, once a week, and more than once a week) were no longer statistically significant.

An analysis of the SEDS-S resulted in findings similar to outcomes on the SEHS-S, where scores ceased having a significant difference and plateaued after reports of skipping school a few times and more. Based on responses to Question 21, students who reported skipping school more than a few times did not have significantly different scores on the SEDS-S (i.e., $a$ few times and once a month; once a month and twice a month; once a month and once a week; twice a month and once a week; twice a month and once a week; twice a month and more than once a week; and once a week and more than once a week). This analysis was similar to outcomes using Question 21 and the SEHS-S to measure subjective well-being. However, the comparison of SEDS-S scores for students who reported skipping once a week and more than once a week did not indicate a significant difference like the SEHS-S scores did.

While these results indicated no statistically significant differences in students' mental health after skipping school more than a few times, the difference between scores on the SEDS-S grew gradually as reports of skipping increased. Overall, the difference in SEDS-S scores between students who reported skipping 0 times and those who reported skipping more than once a week, was approaching a medium effect size (.44). When continuing to compare SEDS-S scores to students who skipped 0 times, effect sizes for once a week (.42), twice a month (.41), and once a month (.35) progressively diminished when students reported less absenteeism. When comparing responses from Question 21, differences in the SEDS-S were relatively smaller than the effect sizes for the SEHS-S. This comparison was most notable when comparing the magnitude of difference in SEDS-S scores (.44) and SEHS-S scores (.79) for students who reported skipping 0 times and more than once a week. The difference witnessed in students' 
subjective well-being (SEHS-S) appeared more substantial than the measure of psychological distress (SEDS-S).

\section{Research Questions 3a-3b and 4a-4b Findings Summary}

Overall, mean scores on both the SEHS-S and SEDS-S demonstrated significant differences as students reported more absenteeism on Questions 19 and 21. Compared to 0 days, missing even just 1 day, 2 days, or 3 or more days of school in the past 30 days resulted in significantly different (lower) reports of subjective well-being and significantly different (higher) reports of psychological distress. Effects sizes for differences in subjective well-being and psychological distress for students who missed 0 days compared to 3 or more days remained small (.36 and .35, respectively). Using Question 21, students who reported skipping school over the past 12 months demonstrated the same apparent pattern of an overall significant decrease of subjective well-being and overall significant increase in psychological distress. However, further analysis of Question 21 provided further insight into the changes to student mental health as they reported skipping more school.

After students reported skipping school more than a few times in the past 12 months, SEHS-S and SEDS-S scores alike were not significantly different when comparing responses for skipping more frequently. For instance, when conducting pairwise comparisons of responses to Question 21, the difference in SEDS-S and SEHS-S scores were significant when comparing students who skipped 0 times and 1-2 times and 1-2 times and a few times. The remaining comparisons (a few times and once a month; once a month and twice a month; twice a month and once a week; and once a week and more than once a week) ceased to demonstrate significant differences in students' SEHS-S and SEDS-S scores- with the exception of SEHS-S scores being significantly different when comparing students who skipped once a week and more than once a 
week. This pattern has been illustrated as a plateau in student mental health measures, for both their subjective well-being and psychological distress.

While student mental health appeared to sustain a chronic impact suspected from patterns of SRB, a clear and significant impact was observed nonetheless. Further findings were revealed when analyzing the overall effect size for difference in mental health for students who reported skipping 0 times and those who reported skipping more than once a week. This comparison yielded nearly a large effect size (.79) for students' SEHS-S scores. However, this same comparison resulted in a substantially smaller difference in SEDS-S scores, with a small effect size (.44). Certainly, an overall change in mental health was observed as students reported skipping (and missing) school more often. However, subjective well-being was revealed a more substantial difference as students skipped school more often. By considering a dual-factor approach, the present study identified subjective well-being as a vital component to understanding the overall mental health of students with SRB.

Patterns of absenteeism become notably problematic and indicative of SRB after a student has missed $25 \%$ of school in a given two-week period or 10 days in a fifteen-week period (Kearney \& Albano, 2018). However, the present study's findings supported research that has suggested an earlier threshold of for identifying SRB in students should be considered after missing $10 \%$ of school over a period of three months (Lyon \& Cotler, 2007). Early identification and subsequent interventions should be considered increasingly relevant as the present findings indicated significant changes in mental health after only a few absences from school. Even more compelling were results that found student mental health changed intermittently as skipping school became more habitual and chronic over 12 months. 
Overall, AIR scores from the SEHS-S and SEDS-S remained in the average range for each group of responses to Questions 19 and 21. The existence of one variable (attendance or mental health struggles) did not always coincide with the presence of the other. Suffice to say that not all students with mental health struggles miss or skip school, and not all students who miss or skip school have mental health struggles. However, students with poor mental health miss and skip school more often than their healthy peers, and mental health changed significantly in groups with higher rates of absenteeism. These findings emphasized the importance of building a comprehensive understanding of mental health that considers subjective well-being in addition to psychopathologies when working with students who present SRB.

Research Question 5: Using groups (a) Complete Mental Health, (b) Symptomatic but Content, (c) Vulnerable, and (d) Troubled from a dual-factor model approach, what percentage of students from each group presented with potential SRB?

\section{Research Question 5 Findings}

Student responses to Questions 19 and 21 were divided into groups based on the dualfactor model, including Complete Mental Health [average to high subjective well-being and low psychopathology], Symptomatic but Content [average to high subjective well-being and high psychopathology], Vulnerable [low subjective well-being and low psychopathology], and Troubled (low subjective well-being and high psychopathology]). Based on results from the present study, groups consistent with the dual-factor model had a significant association, overall, with attendance reports from Questions 19 and 21. These outcomes demonstrated small effect sizes for each question (Cramer's $V=.098 \& .112$, respectively). However, the magnitude of these differences presented a relatively stronger relationship than each of the demographic factors analyzed in Research Questions 2a-2d (Cramer's $V=.022-.089)$. 
Groups of students with progressing mental health struggles were drastically more representative of attendance problems when compared to students with fewer mental health problems. Several students from the Troubled (23.7\%) and Vulnerable (22.1\%) groups reported missing 3 or more days of school in the past 30 days. Comparatively, students in the Symptomatic but Content group (17.9\%) were less represented. Finally, students in the Complete Mental Health group (10.1\%) were least represented. As students indicated lower psychological distress or higher subjective well-being, they reported fewer attendance problems.

In students who were Symptomatic but Content, psychological distress was evident but subdued, potentially, by average to high subjective well-being. Fewer of these students reported having missed 3 or more days of school, insinuating that subjective well-being may be a protective factor against SRB. Additionally, fewer students in the Vulnerable group reported attendance problems when compared to Troubled youth. The Vulnerable group consisted of low subjective well-being despite showing little psychological distress and are considered high-risk for developing psychological distress and developing into being Troubled. These findings served as further evidence of the suspected impact that mental health deterioration can have on attendance when subjective well-being decreases and psychological distress increases.

Question 21 demonstrated similar trends. No more than one percent of students with Complete Mental Health reported having skipped school once a month or more (i.e., twice a month, once a week, more than once a week). This same cut-off point was established previously as a potential threshold where subjective well-being and psychological distress stopped demonstrating a significant difference in students with SRB. Higher rates of absenteeism were consistently reported by students with lower subjective well-being and higher psychological distress- continuing to strengthen the evidence of a connection between mental health and SRB. 
In their study using the dual-factor model to conceptualize mental health, Suldo and Schaffer (2008) identified students in the Complete Mental Health group for exhibiting average to high subjective well-being with low psychopathology. This group of students demonstrated several positive attributes and protective factors, including better attendance. Suldo and Schaffer (2008) eluded to a vital component for understanding student mental health- the role of subjective well-being. More recently, research has identified specific factors of subjective wellbeing as more predictive of attendance problems (Wroblewski et al., 2019). Results from the present study further supported subjective well-being as a protective factor against SRB. Additionally, the difference in reports of subjective well-being was substantially more significant than the difference found in psychological distress for students with SRB.

\section{Strengths and Limitations}

Prior studies have used several different methods and parameters to measure absenteeism. A lack of distinction between terms (e.g., missing or skipping school) has resulted in further incongruencies in research. Consequently, various outcomes have emerged regarding the prevalence, identification methods, and overall understanding of SRB. The present study used separate questions on the CHKS-SCM for missing and skipping school to strengthen its validity and broaden its inclusivity. Additionally, time duration relied on two metrics, including "the past 30 days" as well as "the past 12 months." The present sample size $(n=107,125)$ provided adequate sampling for each response to Questions 19 and 21. Additionally, the CHKS-SCM gathered data from 296 different schools from various settings in California as opposed to other sources commonly used in studies (e.g., truancy courts, drug-related surveys, school crime surveys). 
The present dataset was highly representative of Hispanic/Latinx students (48.1\%) and other diverse student backgrounds. This detail emphasized the rich and diverse data available through the CHKS-SCM and was a strength to the present study. Due to the level of detail elicited from this survey, the present study analyzed several aspects of students' demographic backgrounds. The sample was limited to the age range of secondary students in grades 7-12. A limitation to generalizing results from this study was identified in California being a majorityminority state with a substantial portion of students identifying as a race/ethnicity other than White/Caucasian (Furlong et al., 2020). Additionally, while the relationship between demographic factors and attendance had a significant association, the effect sizes for each of these remained small.

The methods and instruments used throughout analysis were strong and suitable for this study and presented with only some limitations. As previously discussed, Questions 19 and 21 regarding attendance on the CHKS-SCM present different implied meanings for absenteeism (missing or skipping). It was essential to acknowledge a strong likelihood that several students who may have reported frequently missing or skipping school were not in attendance to complete the school-based survey.

The SEHS-S and SEDS-S were relied on in this study to measure subjective well-being and psychological distress, respectively. These instruments have a sound psychometric background with appropriate use in diverse populations and were designed specifically for administration in unison to align with the dual-factor model. The SEHS-S and SEDS-S were limited to their function as screening tools and should supplement additional measures to establish further diagnostic information. Despite heterogeneous outcomes being encountered regularly in previous SRB studies, the SEHS-S and SEDS-S maintained exceptional internal 
consistency reliability even as students reported more frequent absenteeism (see Table 2 in Chapter 4).

Using a dual-factor model approach when analyzing SRB in secondary students was a fundamental strength of the present study. A statistically significant relationship was established between dual-factor groups for mental health and both Questions 19 and 21 on attendance. While the effect sizes for these outcomes remained small, mental health groups had a more substantial relationship with attendance than any demographic factor. Additionally, the effect sizes for changes in subjective well-being were substantial findings when comparing students who skipped 0 times and those who reported skipping once a week or more. Currently, the dual-factor model lacks research supporting its theoretical use in specific populations, such as students with SRB. Additionally, scores in the average range on the SEDS-S excluded students from any dualfactor group as low and high scores determined group membership.

\section{Implications for Practice}

Understanding the mental health changes in students with SRB has remained a complex phenomenon in research. Students with SRB often present subtle or masked symptoms and demonstrate levels of psychopathology that may meet multiple diagnostic criteria or none at all (Bools et al., 1990; Egger et al., 2003; Kearney \& Albano, 2004; Kearney et al., 2005). A persistent heterogeneity has convoluted researchers' understanding of how mental health interacts with cases of SRB. The present study intended to enhance our understanding of the mental health changes in students with SRB by implementing a dual-factor approach. Results indicated that an overall significant difference in mental health was present across students who were absent more frequently. These students had lower reports of subjective well-being and higher reports of 
psychological distress. Most notably, the analysis found drastic differences in students' subjective well-being when they reported missing or skipping school more frequently.

Previous literature has recommended thorough assessment procedures for students with SRB that included medical, familial, and mental health factors. The present study found that employing a dual-factor model approach to understanding mental health was essential for understanding students with SRB. Subjective well-being has a critical and distinct role in their

mental health and may contribute as a protective factor against SRB. While several variables are likely to influence a students' attendance, subjective well-being and psychological distress had a more significant association with attendance outcomes than other demographic factors.

The dual-factor model was paramount in building a better understanding of mental health in students with SRB. Without this perspective, the difference in psychological distress witnessed in students who skipped more school may have been viewed as negligible. By considering the subjective well-being of this population, in addition to traditional psychopathology, practitioners can improve earlier identification, prevention, and intervention methods. However, not all students who presented with attendance problems reported mental health struggles; subsequently, a holistic and comprehensive approach must be maintained in understanding this vulnerable population. The significant role that demographic variables play must also be taken into account. These important contextual components of a student were recognized as playing an important role in attendance and practitioners are encouraged to be mindful of their potential interaction with absenteeism.

\section{Future Directions}

To further expand a comprehensive understanding of students' attendance, research should continue deliberating the role that demographic and ecological factors have. These 
elements of a student's background should be considered, potentially as isolated variables, to understand how specific profiles of SRB may be different. Research can then further explore how a student's age, gender, sexual orientation, race/ethnicity, and socioeconomic background interact with topics such as school climate, academic achievement, positive relationships with peers or staff, and academic engagement. These indicators for school climate are lacking from literature on this topic and warrant further exploration. By establishing what predictive value these variables have with regard to attendance, future studies should determine what early identifiers and protective factors can detect or prevent SRB.

The CHKS and additional modules are available and often obligated for use in California schools. Data from these surveys provide a diverse sample with extensive scales established for school climate and related phenomenon. Future researchers are strongly encouraged to utilize data from the CHKS or similar available datasets when exploring topics regarding school attendance. Based on additional attendance questions from the CHKS-SCM, future research should investigate whether the reasons students reported for their absences interact with their mental health in different ways. For instance, do different profiles exist for students reporting to avoid school because of anxiety instead of hanging out with friends or engaging in substance use? This question and others alike would provide vital information for continuing research on identifying the function behind a student's SRB and its subsequent impact. Lastly, Future research on SRB is encouraged to continue employing a dual-factor model when evaluating students' mental health.

\section{Conclusion}

Chapter 5 provided a summary of findings for each research question following the analyses from the preceding chapter. The present study analyzed an extensive dataset including 
the CHKS-SCM, an optional module measuring subjective well-being (SEHS-S), and an additional measure of psychological distress (SEDS-S). The CHKS-SCM provided two separate questions about attendance that inquired about students missing or skipping school over the past 30 days or the past 12 months. By using both these items, this study avoided some of the previous ambiguity research has faced in the lack of distinction between these functions- missing versus skipping school. The prevalence of students demonstrating a potential pattern of SRB was identified as a range from $4.5-13.5 \%$ in the CHKS-SCM. This range has improved on commonly used rates of prevalence that include $5-28 \%$ of students. It was acknowledged that students with chronic attendance problems might have been unable to complete the CHKS-SCM due to their absence.

The present study confirmed foundational findings from previous studies regarding the vital role that a student's demographic background has in attendance. Results continued to support that girls often skipped and missed school more than their male peers. However, transgender students represented a higher percentage of responses indicative of SRB. Consistent with previous research findings, select students of color reported attendance problems more often when compared to Caucasian/White and Asian students. Lastly, students whose parents had lower education levels and students eligible for free or reduced-price lunches reported SRB more often.

A holistic perspective of mental health should not dismiss the influence of demographic factors. Accordingly, the current study analyzed critical items on the CHKS-SCM related to student demographics. Research questions about students' backgrounds and attendance confirmed previous research findings and acknowledged the vital role that demographic factors have regarding attendance. Ongoing research on this topic has suggested that certain 
demographic features may position students in distinct profiles with varying degrees of risk for attendance problems (Maynard et al., 2017). This notion, and the supporting evidence from the present study, identified absenteeism as an essential topic in need of further research.

A significant component of the present study was using the dual-factor approach to comprehensively analyze changes in student mental health when SRB was present. The SEHS-S (Furlong et al., 2020) and SEDS-S (Dowdy et al., 2018) were designed to be co-administered to assess both subjective well-being and psychological distress. These instruments have excellent psychometric qualities and maintained the dual-factor approach that was unique to the present study. Despite the common heterogeneity encountered in studies of SRB, these measures maintained high internal consistency reliability even as students reported missing or skipping school more frequently.

Analyzing the mental health of students with SRB through the dual-factor lens identified a substantial difference in the subjective well-being of students who missed and skipped more school. Together, both subjective well-being and psychological distress create a more holistic view of mental health. Although student reports indicated a statistically significant difference in their psychological distress scores, the magnitude of this difference $(\Delta=.44)$ was not as substantial as the difference found in their subjective well-being $(\Delta=.79)$. This sizeable effect size outcome suggested that subjective well-being has a pivotal role in the mental health of students with SRB and should be implemented in practice and future research.

While students demonstrated overall differences in their mental health as they missed and skipped more school, further analysis indicated that statistically significant differences were not consistent across responses for skipping school. As students reported skipping once a month and more, their subjective well-being and psychological distress scores were not significantly 
different than each sequential response (twice a month, once a week, more than once a week). These results warrant further research but implied that chronic differences in mental health might have emerged as students skipped more school.

Lastly, students presented statistically significant differences on each mental health measure after missing only 1 day of school or skipping 1-2 times or a few times when compared to missing or skipping 0 days or times. Criteria for establishing concern for SRB have remained arbitrary and lacking guidance for practitioners and researchers. This early indication of reduced subjective well-being and increased psychological distress encouraged future researchers to explore evidence of an earlier threshold for when patterns of SRB may impact students. Based on comparing students in groups established using the dual-factor model, students reported more absenteeism as their reports of subjective well-being decreased and psychological distress increased. These groups demonstrated a significant relationship with attendance and positioned the dual-factor model as a new and critical perspective to understanding mental health in students with SRB. 


\section{References}

Allen, M. (2017). The Sage Encyclopedia of Communication Research Methods_(Vols. 1-4). SAGE Publications, Inc. https://doi.org/10.4135/9781483381411

Almeida, M. C., Aquino, E. M., \& de Barros, A. P. (2006). School trajectory and teenage pregnancy in three Brazilian state capitals. Cadernos de Saude Publica, 22, 1937-1409. https://doi.org/10.1590/s0102-311×2006000700005

American Psychiatric Association (2013). Diagnostic and statistical manual of mental disorders (5th ed.). Author.

Arvans, R. K., \& LeBlanc, L. A. (2009). Functional assessment and treatment of migraine reports and school absences in an adolescent with Asperger's disorder. Education \& Treatment of Children, 32(1), 151-166. https://doi.org/10.1353/etc.0.0046

Atkinson, L., Quarrington, B., \& Cyr, J. J. (1985). School refusal: The heterogeneity of a concept. American Journal of Orthopsychiatry, 55 83-101. https://doi.org/10.1111/j.1939-0025.1985.tb03423.x

Attwood, G., \& Croll, P. (2006). Truancy in secondary school pupils: Prevalence, trajectories, and pupil perspectives. Research papers in Education, 21, 467-484. https://doi.org/10.1080/02671520600942446

Austin, G., Hanson, Y., Bono, G., \& Cheng, Z. (2007). The Achievement Gap, school wellbeing, and learning supports. CHKS Factsheet \#8. WestEd.

Austin, G., O’Malley, M., \& Izu, J. (2011). Making Sense of School Climate: Using the California School Climate, Health, and Learning (Cal-SCHLS) Survey System to Inform Your School Improvement Efforts. WestEd. 
Baams, L., Talmage, C. A., \& Russell, S. T. (2017). Economic costs of bias-based bullying. School Psychology Quarterly, 32(3), 422-433. https://doi.org/10.1037/spq0000211

Becker, E. M., Jensen-Doss, A., Kendall, P. C., Birmaher, B., \& Ginsburg, G. S. (2016). All anxiety is not created equal: Correlates of parent/youth agreement vary across subtypes of anxiety. Journal of Psychopathology and Behavioral Assessment, 38(4), 528-537. https://doi.org/10.1007/s10862-016-9544-z

Belhekar, V. M. A. (2016). Statistics for psychology using R. SAGE Publications.

Bernstein, G. A., \& Borchardt, C. M. (1996). School refusal: Family constellation and family functioning. Journal of Anxiety Disorders, 10(1), 1-19. https://doi.org/10.1016/0887$\underline{6185(95) 00031-3}$

Bernstein, G. A., Massie, E. D., Thuras, P. D., Perwien, A. R., Borchardt, C. M., \& Crosby, R. D. (1997). Somatic symptoms in anxious-depressed school refusers. Journal of the American Academy of Child and Adolescent Psychiatry, 36(5), 661-668. https://doi.org/10.1097/00004583-199705000-00017

Bernstein, G. A., Svingen, P. H., \& Garfinkel, B. D. (1990). School phobia: Patterns of family functioning. Journal of the American Academy of Child \& Adolescent Psychiatry, 29(1), 24-30. https://doi.org/10.1097/00004583-199001000-00005

Bools, C., Foster, J., Brown, I., \& Berg, I. (1990). The identification of psychiatric disorders in children who fail to attend school: a cluster analysis of a non-clinical population. Psychological Medicine, 20(1), 171-181. https://doi.org/10.1017/s0033291700013350 
California Department of Education (2020, December 4) School Attendance Review Boards. https://www.cde.ca.gov/ls/ai/sb/\#: :text=California\%20compulsory\%20education\%20la w\%20requires,obtained\%20parental\%20permission\%20to\%20leave.

CalSCHLS. (2021a, January 21). California Healthy Kids Survey. https://calschls.org/about/thesurveys/\#chks

CalSCHLS. (2021b, January 21). Survey Administration. https://calschls.org/surveyadministration/

Carless, B., Melvin, G. A., Tonge, B. J., \& Newman, L. K. (2015). The role of parental selfefficacy in adolescent school-refusal. Journal of Family Psychology, 29(2), 162-170. https://doi.org/10.1037/fam0000050

Carroll, H. C. M. (2015). Pupil absenteeism and the educational psychologist. Educational Studies, 41(1-2), 47-61. https://doi.org/10.1080/03055698.2014.955731

Chang, H. N., Romero, M., \& National Center for Children in Poverty (2008). Present, Engaged, and Accounted for: The Critical Importance of Addressing Chronic Absence in the Early Grades. https://www.nccp.org/wp-content/uploads/2008/09/text_837.pdf

Chorpita, B. F., Albano, A. M., Heimberg, R. G., \& Barlow, D. H. (1996). A systematic replication of the prescriptive treatment of school refusal behavior in a single subject. Journal of Behavior Therapy and Experimental Psychiatry, 27(3), 281-290. https://doi.org/10.1016/s0005-7916(96)00023-7

Chou, L. -C., Ho, C. -Y., Chen, C. -Y., \& Chen, W. J. (2006). Truancy and illicit drug use among adolescents surveyed via street outreach. Addictive Behaviors, 31(1), 149-154. https://doi.org/10.1016/j.addbeh.2005.04.011 
Christle, C. A., Jolivette, K., \& Nelson, M. (2007). School characteristics related to high school dropout rates. Remedial and Special Education, 28, 325-339. https://doi.org/10.1177/07419325070280060201

Cohen, J. (1988). Statistical power analysis for the behavioral sciences (2nd ed.). Erlbaum

Craun, E. A., Haight, C., DeCou, C. R., Babbitt, S. C., \& Wong, M. M. (2017). The differential relationship among peer group indicators and internalizing symptoms in a problematic absenteeism population. North American Journal of Psychology, 19(2), 485-498.

Daleiden, E. L., Chorpita, B. F., Kollins, S. H., \& Drabman, R. S. (1999). Factors affecting the reliability of clinical judgments about the function of children's school-refusal behavior. Journal of Clinical Child Psychology, 28(3), 396-406. https://doi.org/10.1207/S15374424jecp280312

Delgado, B., Martinez-Monteagudo, M. C., Ruiz-Esteban, C., \& Rubio, E. (2019). Latent class analysis of school refusal behavior and its relationship with cyberbullying during adolescence. Frontiers in Psychology, 10, 1916. https://doi.org/10.3389/fpsyg.2019.01916

Diener, E. (2000). Subjective well-being: The science of happiness and a proposal for a national index. American Psychologist, 55, 34-43. https://doi.org/10.1037/0003-066X.55.1.34

Dowdy, E., Furlong, M. J., Nylund-Gibson, K., Moore, S., \& Moffa, K. (2018). Initial validation of the Social Emotional Distress Scale to support complete mental health screening. Assessment for Effective Intervention, 43, 241-248. https://doi.org/10.1177/1534508417749871 
Furlong, M. J. (2021). Examination of the Social Emotional Distress Survey-Secondary for Use Across Sociocultural Groups [Manuscript submitted for publication]. Department of counseling, clinical, and school psychology, University of California Santa Barbara.

Dube, S. R., \& Orpinas, P. (2009). Understanding excessive school absenteeism as school refusal behavior. Children \& Schools, 31(2), 87-95. https://doi.org/10.1093/cs/31.2.87

Durlak, J. A (2009). How to select, calculate, and Interpret effect sizes, Journal of Pediatric Psychology, 34(9), 917-928. https://doi.org/10.1093/jpepsy/jsp004

Egger, H. L., Costello, E. J., \& Angold, A. (2003). School refusal and psychiatric disorders: A community study. Journal of the American Academy of Child and Adolescent Psychiatry, 42(7), 797-807. https://doi.org/10.1097/01.CHI.0000046865.56865.79

Elliott, J. G. (1999). School refusal: Issues of conceptualisation, assessment, and treatment. Journal of Child Psychology and Psychiatry, and Allied Disciplines, 40(7), 1001-1012.

Elliott, J. G., \& Place, M. (2019). Practitioner Review: School refusal: Developments in conceptualisation and treatment since 2000. Journal of Child Psychology and Psychiatry, and Allied Disciplines, 60(1), 4-15. https://doi.org/10.1111/jepp.12848

Frey, B. (2018). The SAGE encyclopedia of educational research, measurement, and evaluation (Vols. 1-4). SAGE Publications, Inc. https://doi.org/10.4135/9781506326139

Furlong, M. J., Dowdy, E., Carnazzo, K., Bovery, B., \& Kim, E. (2014). Covitality: Fostering the building blocks of complete mental health. Communiqué, 42(8), 28-29.

Furlong, M. J., Nylund-Gibson, K., Dowdy, E., Wagle, R., Hinton, T., \& Carter, D. (2020). Modification and standardization of Social Emotional Health Survey-Secondary, 2020 
edition. Santa Barbara, CA, University of California Santa Barbara, International Center for School Based Youth Development.

Furlong, M. J., You, S., Renshaw, T. L., O’Malley, M. D., \& Rebelez, J. (2013). Preliminary development of the Positive Experiences at School Scale for elementary school children. Child Indicators Research, 6, 753-775. https://doi.org/10.1007/s12187-013-9193-7

Furlong, M. J., You, S., Renshaw, T. L., Smith, D. C., \& O’Malley, M. D. (2014). Preliminary development and validation of the Social and Emotional Health Survey for secondary students. Social Indicators Research, 117, 1011-1032. https://doi.org/10.1007/s11205$\underline{013-0373-0}$

Garland, E. J. (2001). Rages and refusals: Managing the many faces of adolescent anxiety. Canadian Family Physician, 47, 1023-1030.

Gennetian, L. A., Rodrigues, C., Hill, H. D., \& Morris, P. A. (2018). Stability of income and school attendance among NYC students of low-income families. Economics of Education Review, 63, 20-30. https://doi.org/10.1016/j.econedurev.2018.01.003

Gentle-Genitty, C., Karikari, I., Chen, H., Wilka, E., \& Kim, J. (2015). Truancy: A look at definitions in the USA and other territories. Educational Studies, 41, 62-90. https://doi.org/10.1080/03055698.2014.955734

Gibler, R. C., Beckmann, E. A., Lynch-Jordan, A. M., Kashikar-Zuck, S., \& Mano, K. E. J. (2019). Characterizing social and academic aspects of school anxiety in pediatric chronic pain. The Clinical Journal of Pain, 35(7), 625-632. https://doi.org/10.1097/AJP.0000000000000720

Gonzálvez, C., Díaz-Herrero, Á., Sanmartín, R., Vicent, M., Pérez-Sánchez, A. M., \& GarcíaFernández, J. M. (2019). Identifying risk profiles of school refusal behavior: Differences 
in social anxiety and family functioning among Spanish adolescents. International Journal of Environmental Research and Public Health, 16(19). https://doi.org/10.3390/ijerpH16193731

Gonzálvez, C., Inglés, C.J., Kearney, C.A., Vicent, M., Sanmartín, R., \& García-Fernández, J.M. (2016). School refusal assessment scale-revised: Factorial invariance and latent means differences across gender and age in Spanish children. Frontiers in Psychology, 7, 2011. https://doi.org/10.3389/fpsyg.2016.02011

Gonzálvez, C., Kearney, C. A., Jiménez-Ayala, C. E., Sanmartín, R., Vicent, M., Inglés, C. J., \& García-Fernández, J. M. (2018). Functional profiles of school refusal behavior and their relationship with depression, anxiety, and stress. Psychiatry Research, 269, 140-144. https://doi.org/10.1016/j.psychres.2018.08.069

Gonzálvez, C., Sanmartín, R., Vicent, M., Inglés, C. J., Aparicio-Flores, M. P., \& GarcíaFernández, J. M. (2018). Academic self-attributions for success and failure in mathematics and school refusal. Psychology in the Schools, 55(4), 366-376. https://doi.org/10.1002/pits.22117

Gottfried, M. A. (2014). Chronic absenteeism and its effects on students' academic and socioemotional outcomes. Journal of Education for Students Placed at Risk, 19, 53-75. https://doi.org/10.1080/10824669.2014.962696

Greenspoon, P. J., \& Saklofske, D. H. (2001). Toward an integration of subjective well-being and psychopathology. Social Indicators Research, 54, 81-108. https://doi.org/10.1023/A:1007219227883

Haight, C., Kearney, C. A., Hendron, M., \& Schafer, R. (2011). Confirmatory analyses of the School Refusal Assessment Scale-Revised: Replication and extension to a truancy 
sample. Journal of Psychopathology and Behavioral Assessment, 33(2), 196-204. https://doi.org/10.1007/s10862-011-9218-9

Hansen, C., Sanders, S. L., Massaro, S., \& Last, C. G. (1998). Predictors of severity of absenteeism in children with anxiety-based school refusal. Journal of Clinical Child Psychology, 27(3), 246-254. https://doi.org/10.1207/s15374424jccp2703_2

Hanson, T. (2011, December). Measurement analysis of CHKS Core and School Climate Modules items. Paper prepared for the California Safe and Supportive Schools Project. San Francisco: WestEd. https://calschls.org/reports-data/legacy/\#psychometric_studies

Hanson, T. (2012, September). Measurement of School Climate, Health, and Learning Survey for Parents. Paper prepared for the California Safe and Supportive Schools Project. San Francisco: WestEd. https://calschls.org/reports-data/legacy/\#psychometric_studies

Hanson, T., \& Kim, J-O. (2007). Measuring the psychometric properties of the California Healthy Kids Resilience and Youth Development Module. Regional Educational Laboratory West, Report REL 2007-No. 034. WestEd: San Francisco. https://ies.ed.gov/ncee/edlabs/regions/west/pdf/REL_2007034.pdf

Hanson, T., \& Voight, A. (2014). The appropriateness of a California student and staff survey for measuring middle school climate (REL 2014-039). Washington, DC: U.S. Department of Education, Institute of Education Sciences, National Center for Education Evaluation and Regional Assistance, Regional Educational Laboratory West. https://ies.ed.gov/ncee/edlabs/projects/project.asp?projectID $=323$

Henry, K. L. (2007). Who's skipping school: characteristics of truants in 8th and 10th grade. Journal of School Health, 77, 29-35. https://doi.org/10.1111/j.1746-1561.2007.00159.x 
Henry, K. L., \& Huizinga, D. H. (2007). Truancy's effect on the onset of drug use among urban adolescents placed at risk. Journal of Adolescent Health, 40(4), e9-e17. https://doi.org/10.1016/j.jadohealth.2006.11.138

Hersov, L. (1990). School refusal: An overview. In C. Chiland \& J. G. Young (Eds.), Why children reject school: Views from seven countries (pp. 16-41). Yale University Press. https://doi.org/10.1097/00004583-199203000-00036

Heyne, D., \& Sauter, F. M. (2013). School refusal. In C. C. Essau \& T. H. Ollendick (Eds.), The Wiley-Blackwell handbook of the treatment of childhood and adolescent anxiety (pp. $471-$ 517). Wiley.

Heyne, D., Gren-Landell, M., Melvin, G., \& Gentle-Genitty, C. (2019). Differentiation between school attendance problems: Why and how? Cognitive and Behavioral Practice, 26(1), 8-34. https://doi.org/10.1016/j.cbpra.2018.03.006

Hochadel, J., Frölich, J., Wiater, A., Lehmkuhl, G., \& Fricke-Oerkermann, L. (2014). Prevalence of sleep problems and relationship between sleep problems and school refusal behavior in school-aged children in children's and parents' ratings. Psychopathology, 47(2), 119126. https://doi.org/10.1159/000345403

Honjo, S., Nishide, T., Niwa, S., Sasaki, Y., Kaneko, H., Inoko, K., \& Nishide, Y. (2001). School refusal and depression with school attendance in children and adolescents: Comparative assessment between the Children's Depression Inventory and somatic complaints. Psychiatry and Clinical Neurosciences, 55(6), 629-634. https://doi.org/10.1046/j.1440-1819.2001.00916.x

Honjo, S., Sasaki, Y., Kaneko, H., Tachibana, K., Murase, S., Ishii, T., Nishide, Y., \& Nishide, T. (2003). Study on feelings of school avoidance, depression, and character tendencies 
among general junior high and high school students. Psychiatry and Clinical Neurosciences, 57(5), 464-471. https://doi.org/10.1046/j.1440-1819.2003.01149.x

Individuals with Disabilities Education Act, 20 U.S.C. § 1400 (2004). https://sites.ed.gov/idea/

Inglés, C. J., Gonzálvez-Maciá, C., García-Fernández, J. M., Vicent, M., \& MartínezMonteagudo, M. C. (2015). Current status of research on school refusal. European Journal of Education and Psychology, 8(1), 37-52. https://doi.org/10.1016/j.ejeps.2015.10.005

Johnston, J. E., Berry, K. J., \& Mielke, P. W. (2004). A measure of effect size for experimental designs with heterogeneous variances. Perceptual and Motor Skills 98, 3-18. https://doi.org/10.2466/pms.98.1.3-18

Kearney, C. A (2001). School refusal behavior in youth: A functional approach to assessment and treatment. American Psychological Association.

Kearney, C. A. (2002a). Case study of the assessment and treatment of a youth with multifunction school refusal behavior. Clinical Case Studies, 1(1), 67-80. https://doi.org/10.1177/1534650102001001006

Kearney, C.A. (2002b). Identifying the function of school refusal behavior: A revision of the School Refusal Assessment Scale. Journal of Psychopathology and Behavioral Assessment, 24, 235-245. https://doi.org/10.1023/A:1020774932043

Kearney, C.A. (2003). Bridging the gap among professionals who address youth with school absenteeism: Overview and suggestions for consensus. Professional Psychology: Research and Practice, 34, 57-65. https://doi.org/10.1037/0735-7028.34.1.57 
Kearney, C. A. (2007). Forms and functions of school refusal behavior in youth: An empirical analysis of absenteeism severity. Journal of Child Psychology and Psychiatry, 48(1), 5361. https://doi.org/10.1111/j.1469-7610.2006.01634.x

Kearney, C. A. (2008). School absenteeism and school refusal behavior in youth: A contemporary review. Clinical Psychology Review, 28(3), 451-471. https://doi.org/10.1016/j.cpr.2007.07.012

Kearney, C. A. \& Albano, A. M. (2004). The functional profiles of school refusal behavior: Diagnostic aspects. Behavior Modification, 28(1), 147-161. https://doi.org/10.1177/0145445503259263

Kearney, C. A., \& Albano, A. M. (2018). When Children Refuse School: A cognitive-behavioral therapy approach, 3rd. Oxford University Press.

Kearney, C. A. \& Silverman, W. K. (1988). Measuring the function of school refusal behavior: The School Refusal Assessment Scale (SRAS). Paper presented at the meeting of the Association for Advancement of Behavior Therapy, New York.

Kearney, C. A., \& Silverman, W. K. (1990). A preliminary analysis of a functional model of assessment and treatment for school refusal behavior. Behavior Modification, 14(3), 340366. https://doi.org/10.1177/01454455900143007

Kearney, C. A., \& Silverman, W. K. (1993). Measuring the function of school refusal behavior: The School Refusal Assessment Scale. Journal of Clinical Child Psychology, 22(1), 8596. https://doi.org/10.1207/s15374424jccp2201_9

Kearney, C. A., \& Silverman, W. K. (1996). The evolution and reconciliation of taxonomic strategies for school refusal behavior. Clinical Psychology: Science and Practice, 3(4), 339-354. https://doi.org/10.1111/j.1468-2850.1996.tb00087.x 
Kearney, C. A., Chapman, G., \& Cook, L. C. (2005). School refusal behavior in young children. International Journal of Behavioral Consultation and Therapy, 1(3), 216-222. https://doi.org/10.1037/H1100746

Kearney, C. A., Lemos, A., \& Silverman, J. (2004). The functional assessment of school refusal behavior. Behavior Analyst Today, 5(3), 275-283. https://doi.org/10.1037/h0100040

Kearney, C.A., \& Silverman, W.K. (1999). Functionally-based prescriptive and nonprescriptive treatment for children and adolescents with school refusal behavior. Behavior Therapy, 30, 673-695. https://doi.org/10.1016/S0005-7894(99)80032-X

Kena, G., Hussar W., McFarland J., de Brey C., Musu-Gillette, L., Wang, X., Zhang, J., Rathbun, A., WilkinsonFlicker, S., Diliberti M., Barmer, A., Bullock Mann, F., and Dunlop Velez, E. (2016). The Condition of Education 2016 (NCES 2016-144). U.S. Department of Education, National Center for Education Statistics. Washington, DC. https://nces.ed.gov/pubsearch.

Keppens, G., \& Spruyt, B. (2017). Towards a typology of occasional truancy: An operationalization study of occasional truancy in secondary education in Flanders. Research Papers in Education, 32, 121-135. https://doi.org/10.1080/02671522.2015.1136833

Kessler, Ronald C. National Comorbidity Survey: Adolescent Supplement (NCS-A), 2001-2004. Inter-university Consortium for Political and Social Research [distributor], 2017-01-18. https://doi.org/10.3886/ICPSR28581.v6

King, N. J., \& Bernstein, G. A. (2001). School refusal in children and adolescents: A review of the past 10 years. Journal of The American Academy of Child and Adolescent Psychiatry, 40(2), 197-205. https://doi.org/10.1097/00004583-200102000-00014 
Lakens, D. (2013). Calculating and reporting effect sizes to facilitate cumulative science: A practical primer for t-tests and ANOVAs. Frontiers in Psychology, 4. https://doi.org/10.3389/fpsyg.2013.00863

Last, C. G. (1991). Somatic complaints in anxiety disordered children. Journal of Anxiety Disorders, 5(2), 125-138. https://doi.org/10.1016/0887-6185(91)90024-N

Last, C. G., \& Francis, G. (1988). School phobia. In B. B. Lahey \& A. E. Kazdin (Eds.), Advances in clinical child psychology, (Vol. 11, pp. 193-222). Plenum Press.

Lauchlan, F. (2003). Responding to chronic non-attendance: A Review of intervention approaches. Educational Psychology in Practice, 19, 133-146. https://doi.org/10.1080/02667360303236

Lee, K. K. S., Chong, J. Q., \& Abu Bakar, A. K. (2018). School refusal in adolescents with systemic lupus erythematosus (SLE): A case series. Asian Journal of Psychiatry, 34, 5960. https://doi.org/10.1016/j.ajp.2018.04.021

Lee, S., You, S., \& Furlong, M. J. (2016). Validation of the Social Emotional Health Survey for Korean school students. Child Indicators Research, 9, 73-92. https://doi.org/10.1007/s12187-014-9294-y

Lenzi, M., Furlong, M. J., Dowdy, E., Sharkey, J. D., Gini, G., \& Altoè, G. (2015). The quantity and variety across domains of psychological and social assets associated with school victimization. Psychology of Violence, 5, 411-421. https://doi.org/10.1037/a0039696

Lenzi, M., Sharkey, J., Wroblewski, A., Furlong, M. J., \& Santinello., M. (2019). Protecting youth from gang membership: Individual and school-level emotional competence. Journal of Community Psychology, 47, 563-578. https://doi.org/10.1002/jcop.22138 
Lewis-Beck, M. S., Bryman, A., \& Futing Liao, T. (2004). The SAGE encyclopedia of social science research methods. SAGE Publications. https://doi.org/10.4135/9781412950589

Lyon, A. R., \& Cotler, S. (2007). Toward reduced bias and increased utility in the assessment of school refusal behavior: The case for diverse samples and evaluations of context. Psychology in the Schools, 44, 551-565. https://doi.org/10.1002/pits.20247

Mahecha, J., \& Hanson, T. (2020). Measurement Structure of the California School Climate, Health, and Learning Surveys: Student, Staff, and Parent Surveys. WestEd.

Malcolm, H., Wilson, V., Davidson, J., \& Kirk, S. (2003). Absence from school: A study of its causes and effect in seven LEAs. Queen's Printers.

Maric, M., Heyne, D. A., de Heus, P., van Widenfelt, B. M., \& Westenberg, P. M. (2012). The role of cognition in school refusal: an investigation of automatic thoughts and cognitive errors. Behavioural And Cognitive Psychotherapy, 40(3), 255-269. https://doi.org/10.1017/S1352465811000427

Maynard, B. R., Vaughn, M. G., Nelson, E. J., Salas-Wright, C. P., Heyne, D. A., \& Kremer, K. P. (2017). Truancy in the United States: Examining temporal trends and correlates by race, age, and gender. Children and Youth Services Review, 81, 188-196. https://doi.org/10.1016/j.childyouth.2017.08.008

McShane, G., Walter, G., \& Rey, J. M. (2004). Functional Outcome of Adolescents with "School Refusal." Clinical Child Psychology and Psychiatry, 9(1), 53-60.

\section{https://doi.org/10.1177/1359104504039172}

Moher, D., Liberati, A., Tetzlaf, J., \& Altman, D. G. (2009). Preferred reporting items for systematic reviews and meta-analyses: The PRISMA statement. Annals of Internal Medicine, 151(4), 264-269. https://doi.org/10.7326/0003-4819-151-4-200908180-00135 
Muthén, B. and Kaplan, D. (1992), A comparison of some methodologies for the factor analysis of non-normal Likert variables: A note on the size of the model. British Journal of Mathematical and Statistical Psychology, 45, 19-30. https://doi.org/10.1111/j.2044$\underline{8317.1992 . t b 00975 . x}$

National Center for Education Statistics (NCES) (2021, February 24). Number of Instructional Days and Hours in the School Year, by State: 2018. https://nces.ed.gov/programs/statereform/tab5 14.asp

Nayak, A., Sangoi, B., \& Nachane, H. (2018). School Refusal Behavior in Indian Children: Analysis of Clinical Profile, Psychopathology and Development of a Best-Fit Risk Assessment Model. Indian Journal of Pediatrics, 85(12), 1073-1078. https://doi.org/10.1007/s12098-018-2631-2

Orcan, F. (2020). Parametric or Non-Parametric: Skewness to Test Normality for Mean Comparison. International Journal of Assessment Tools in Education, 7(2), 255-265. https://doi.org/10.21449/ijate.656077

Pagano, R. R. (2009). Understanding statistics in the behavioral sciences (9th ed.). Wadsworth Cengage Learning.

Pampati, S., Andrzejewski, J., Sheremenko, G., Johns, M., Lesesne, C. A., \& Rasberry, C. N. (2020). School Climate among Transgender High School Students: An Exploration of School Connectedness, Perceived Safety, Bullying, and Absenteeism. Journal of School Nursing, 36(4), 293-303. https://doi.org/10.1177/1059840518818259

Pellegrini, D. W. (2007). School non-attendance: Definitions, meanings, responses, interventions. Educational Psychology in Practice, 23(1), 63-77. https://doi.org/10.1080/02667360601154691 
Phelps, L., Cox, D., \& Bajorek, E. (1992). School phobia and separation anxiety: Diagnostic and treatment comparisons. Psychology in the Schools, 29, 384-394.

https://doi.org/10.1002/1520-6807(199210)29:4<384::AID-PITS2310290412>3.0.CO;2-

$\underline{1}$

Prabhuswamy, M., Srinath, S., Girimaji, S., \& Seshadri, S. (2007). Outcome of children with school refusal. Indian Journal of Pediatrics, 74(4), 375-379. https://doi.org/10.1007/s12098-007-0063-5

Pritchard, C., \& Ward, R. I. (1974). The family dynamics of school phobics. British Journal of Social Work, 4(1), 61-94.

Project CoVitality University California Santa Barbara (2020, November 20). Research Timeline Infographic. https://www.covitalityucsb.info/research.html

Project Covitality. (2021a, January 21). SEHS measures. https://www.covitalityucsb.info/sehsmeasures/index.html

Project Covitality. (2021b, January 21). Research. https://www.covitalityucsb.info/research.html

Rayner, S., \& Riding, R. (1996). Cognitive style and school refusal. Educational Psychology, 16(4), 445-451. https://doi.org/10.1080/0144341960160409

Robinson, J. P., \& Espelage, D. L. (2011). Inequities in Educational and Psychological Outcomes between LGBTQ and Straight Students in Middle and High School. Educational Researcher, 40(7), 315-330. https://doi.org/10.3102/0013189X11422112

Salkind, N. J. (2007). Encyclopedia of measurement and statistics (Vols. 1-0). Sage Publications, Inc. https://doi.org/10.4135/9781412952644 
Salkind, N. J. (2010). Encyclopedia of research design (Vols. 1-0). SAGE Publications, Inc. https://doi.org/10.4135/9781412961288

Seçer, I. (2014). The adaptation of School Refusal Assessment Scale into Turkish: Reliability and validity studies. Pakistani Journal of Statistics, 30, 1197-1202.

Sirkin, R. M. (2006). The chi-square test. In Statistics for the social sciences (pp. 397-442). SAGE Publications, Inc. https://www.doi.org/10.4135/9781412

Suldo, S. M., \& Shaffer, E. J. (2008). Looking beyond Psychopathology: The Dual-Factor Model of Mental Health in Youth. School Psychology Review, 37(1), 52-68.

Telef, B. B., \& Furlong, M. J., (2017). Adaptation and validation of the Social Emotional Health Survey-Secondary into Turkish culture. International Journal of School \& Educational Psychology, 5, 255-265. https://doi.org/10.1080/21683603.2016.1234988

Tolin, D. F., Whiting, S., Maltby, N., Diefenbach, G. J., Lothstein, M. A., Hardcastle, S., Catalano, A., \& Gray, K. (2009). Intensive (daily) behavior therapy for school refusal: A multiple baseline case series. Cognitive and Behavioral Practice, 16(3), 332-344. https://doi.org/10.1016/j.cbpra.2009.02.003

Triano, S. L. (2000). Categorical eligibility for special education: The enshrinement of the medical model in disability policy. Disability Studies Quarterly, 20(4). 1-16. https://doi.org/10.18061/dsq.v20i4.263

U.S. Department of Justice, Bureau of Justice Statistics, School Crime Supplement (SCS) to the National Crime Victimization Survey (NCVS), 2007. https://nces.ed.gov/pubs2011/2011316.pdf 
Valles, E., \& Oddy, M. (1984). The influence of a return to school on the long-term adjustment of school refusers. Journal of Adolescence, 7(1), 35-44. https://doi.org/10.1016/0140$\underline{1971(84) 90046-0}$

Vanheule, S., Desmet, M., Meganck, R., Inslegers, R., Willemsen, J., De Schryver, M., \& Devisch, I. (2014). Reliability in psychiatric diagnosis with the DSM: Old wine in new barrels. Psychotherapy and Psychosomatics, 83, 313-314. https://doi.org/10.1159/000358809

Vaughn, M. G., Maynard, B. R., Salas-Wright, C. P., Perron, B. E., \& Abdon, A. (2013). Prevalence and correlates of truancy in the US: Results from a national sample. Journal of Adolescence, 36(4), 767-776. https://doi.org/10.1016/j.adolescence.2013.03.015

Walter, D., von Bialy, J., von Wirth, E., \& Doepfner, M. (2017). Psychometric properties of the German School Refusal Assessment Scale-Revised. Journal of Psychoeducational Assessment, 36, 644-648. https://doi.org/10.1177/0734282916689641

Wang, C., Yang, C., Jiang, X., \& Furlong, M. (2018). Validation of the Chinese version of the Social Emotional Health Survey-Primary. International Journal of School \& Educational Psychology, 6(1), 62-74. https://doi.org/10.1080/21683603.2016.1272026

WestEd (2011). Measurement analysis of CHKS Core and School Climate Module items. https://data.calschls.org/resources/SCM.pdf

Wimmer, M. (2010). School refusal: Information for educators. In Canter, A. S., Paige, L. Z., Roth, M. D., Romero, I., Carroll, S. A., \& National Association of School Psychologists (2010). Helping Children at Home and School III: Handouts for Families and Educators. National Association of School Psychologists (NASP). 
Wood Jeffrey J., Lynne-Landsman Sarah D., Langer David A., Wood Patricia A., Clark Shaunna L., Eddy J. Mark, \& Ialongo Nick. (2012). School Attendance Problems and Youth Psychopathology: Structural Cross-Lagged Regression Models in Three Longitudinal Data Sets. Child Development, 83(1), 351-366. https://doi.org/10.1111/j.1467$\underline{8624.2011 .01677 . x}$

World Health Organization (2004). ICD-10: International statistical classification of diseases and related health problems. World Health Organization.

Wroblewski, A. P., Dowdy, E., Sharkey, J. D., \& Kim, E. K. (2019). Social-emotional screening to predict truancy severity: Recommendations for educators. Journal of Positive Behavior Interventions, 21(1), 19-29. https://doi.org/10.1177/1098300718768773

You, S., Furlong, M. J., Dowdy, E., Renshaw, T. L., Smith, D. C., \& O’Malley, M. D. (2014). Further validation of the social and emotional health survey for high school students. Applied Research in Quality of Life, 9, 997-1015. https://doi.org/10.1007/s11482-013$\underline{9282-2}$

You, S., Furlong, M. J., Felix, E., \& O’Malley, M. D. (2015). Validation of the Social and Emotional Health Survey for five sociocultural groups: Multigroup invariance and latent mean analyses. Psychology in the Schools, 52, 349-362. https:// doi.org/10.1002/pits.21828/abstract

Zheng, H., Larson, D., \& Hanson, T. (2017). Secondary CHKS Scoring Key, 2016-17. WestEd. 


\section{APPENDICES}

\section{Appendix A}

Question 19. In the past $\underline{30 \text { days, }}$, how often did you miss an entire day of school for any reason?
A. I did not miss any days of school in the past 30 days
B. 1 day
C. 2 days
D. 3 or more days

Question 21. During the past $\underline{12}$ months, about how many times did you skip school or cut classes?
A. 0 times
E. Twice a month
B. 1-2 times
F. Once a week
C. A few times
G. More than once a week
D. Once a month 


\section{Appendix B}

\section{Social-Emotional Health Survey-Secondary (SEHS-S): Grades 7-12 (Page 1/2)}

Directions: You are being asked to take a survey about how you have felt over the past few weeks. Your school is doing this survey to better understand your school experiences. With this information, your school wants to provide support to help improve your school experiences.

\begin{tabular}{|c|c|c|c|c|c|}
\hline \multicolumn{2}{|r|}{$\begin{array}{l}\text { Read each item and choose the response that best describes } \\
\text { you. Please respond honestly. There are no right or wrong } \\
\text { answers. You can skip questions you don't want to answer. }\end{array}$} & \multirow{2}{*}{$\begin{array}{l}\begin{array}{c}\text { Not at } \\
\text { all true }\end{array} \\
\text { Not at } \\
\text { all true }\end{array}$} & \multirow{2}{*}{$\begin{array}{c}\begin{array}{c}\text { A little } \\
\text { true }\end{array} \\
\begin{array}{c}\text { A little } \\
\text { true }\end{array}\end{array}$} & \multirow{2}{*}{$\begin{array}{c}\begin{array}{c}\text { Pretty } \\
\text { much true }\end{array} \\
\begin{array}{c}\text { Pretty } \\
\text { much true }\end{array}\end{array}$} & \multirow{2}{*}{$\begin{array}{c}\begin{array}{c}\text { Very } \\
\text { much true }\end{array} \\
\begin{array}{c}\text { Very much } \\
\text { true }\end{array}\end{array}$} \\
\hline 1 & I can work out my problems. & & & & \\
\hline 2 & I can do most things if I try & $\begin{array}{l}\text { Not at } \\
\text { all true }\end{array}$ & $\begin{array}{c}\text { A little } \\
\text { true }\end{array}$ & $\begin{array}{c}\text { Pretty } \\
\text { much true }\end{array}$ & $\begin{array}{l}\text { Very much } \\
\text { true }\end{array}$ \\
\hline 3 & There are many things that I do well. & $\begin{array}{l}\text { Not at } \\
\text { all true }\end{array}$ & $\begin{array}{c}\text { A little } \\
\text { true }\end{array}$ & $\begin{array}{c}\text { Pretty } \\
\text { much true }\end{array}$ & $\begin{array}{l}\text { Very much } \\
\text { true }\end{array}$ \\
\hline 4 & There is a purpose to my life. & $\begin{array}{l}\text { Not at } \\
\text { all true }\end{array}$ & $\begin{array}{l}\text { A little } \\
\text { true }\end{array}$ & $\begin{array}{c}\text { Pretty } \\
\text { much true }\end{array}$ & $\begin{array}{l}\text { Very much } \\
\text { true }\end{array}$ \\
\hline 5 & I understand why I do what I do & $\begin{array}{l}\text { Not at } \\
\text { all true }\end{array}$ & $\begin{array}{c}\text { A little } \\
\text { true }\end{array}$ & $\begin{array}{c}\text { Pretty } \\
\text { much true }\end{array}$ & $\begin{array}{l}\text { Very much } \\
\text { true }\end{array}$ \\
\hline 6 & I understand my moods and feelings. & $\begin{array}{l}\text { Not at } \\
\text { all true }\end{array}$ & $\begin{array}{c}\text { A little } \\
\text { true }\end{array}$ & $\begin{array}{c}\text { Pretty } \\
\text { much true }\end{array}$ & $\begin{array}{l}\text { Very much } \\
\text { true }\end{array}$ \\
\hline 7 & $\begin{array}{l}\text { When I do not understand something, I ask the teacher } \\
\text { again and again until I understand. }\end{array}$ & $\begin{array}{l}\text { Not at } \\
\text { all true }\end{array}$ & $\begin{array}{c}\text { A little } \\
\text { true }\end{array}$ & $\begin{array}{c}\text { Pretty } \\
\text { much true }\end{array}$ & $\begin{array}{l}\text { Very much } \\
\text { true }\end{array}$ \\
\hline 8 & I try to answer all the questions asked in class. & $\begin{array}{l}\text { Not at } \\
\text { all true }\end{array}$ & $\begin{array}{c}\text { A little } \\
\text { true }\end{array}$ & $\begin{array}{c}\text { Pretty } \\
\text { much true }\end{array}$ & $\begin{array}{l}\text { Very much } \\
\text { true }\end{array}$ \\
\hline 9 & $\begin{array}{l}\text { When I try to solve a math problem, I will not stop until I } \\
\text { find a final solution. }\end{array}$ & $\begin{array}{l}\text { Not at } \\
\text { all true }\end{array}$ & $\begin{array}{c}\text { A little } \\
\text { true }\end{array}$ & $\begin{array}{c}\text { Pretty } \\
\text { much true }\end{array}$ & $\begin{array}{l}\text { Very much } \\
\text { true }\end{array}$ \\
\hline 10 & $\begin{array}{l}\text { At my school, there is a teacher or some other adult who } \\
\text { always wants me to do my best. }\end{array}$ & $\begin{array}{l}\text { Not at } \\
\text { all true }\end{array}$ & $\begin{array}{c}\text { A little } \\
\text { true }\end{array}$ & $\begin{array}{c}\text { Pretty } \\
\text { much true }\end{array}$ & $\begin{array}{l}\text { Very much } \\
\text { true }\end{array}$ \\
\hline 11 & $\begin{array}{l}\text { At my school, there is a teacher or some other adult who } \\
\text { listens to me when I have something to say. }\end{array}$ & $\begin{array}{l}\text { Not at } \\
\text { all true }\end{array}$ & $\begin{array}{c}\text { A little } \\
\text { true }\end{array}$ & $\begin{array}{c}\text { Pretty } \\
\text { much true }\end{array}$ & $\begin{array}{l}\text { Very much } \\
\text { true }\end{array}$ \\
\hline 12 & $\begin{array}{l}\text { At my school, there is a teacher or some other adult who } \\
\text { believes that I will be a success. }\end{array}$ & $\begin{array}{l}\text { Not at } \\
\text { all true }\end{array}$ & $\begin{array}{l}\text { A little } \\
\text { true }\end{array}$ & $\begin{array}{c}\text { Pretty } \\
\text { much true }\end{array}$ & $\begin{array}{l}\text { Very much } \\
\text { true }\end{array}$ \\
\hline 13 & My family members really help and support one another. & $\begin{array}{l}\text { Not at } \\
\text { all true }\end{array}$ & $\begin{array}{c}\text { A little } \\
\text { true }\end{array}$ & $\begin{array}{c}\text { Pretty } \\
\text { much true }\end{array}$ & $\begin{array}{c}\text { Very much } \\
\text { true }\end{array}$ \\
\hline 14 & My family really gets along well with each other. & $\begin{array}{l}\text { Not at } \\
\text { all true }\end{array}$ & $\begin{array}{l}\text { A little } \\
\text { true }\end{array}$ & $\begin{array}{c}\text { Pretty } \\
\text { much true }\end{array}$ & $\begin{array}{l}\text { Very much } \\
\text { true }\end{array}$ \\
\hline 15 & There is a feeling of togetherness in my family. & $\begin{array}{l}\text { Not at } \\
\text { all true }\end{array}$ & $\begin{array}{l}\text { A little } \\
\text { true }\end{array}$ & $\begin{array}{c}\text { Pretty } \\
\text { much true }\end{array}$ & $\begin{array}{l}\text { Very much } \\
\text { true }\end{array}$ \\
\hline 16 & I have a friend my age who really cares about me. & $\begin{array}{l}\text { Not at } \\
\text { all true }\end{array}$ & $\begin{array}{c}\text { A little } \\
\text { true }\end{array}$ & $\begin{array}{c}\text { Pretty } \\
\text { much true }\end{array}$ & $\begin{array}{l}\text { Very much } \\
\text { true }\end{array}$ \\
\hline 17 & $\begin{array}{l}\text { I have a friend my age who talks with me about my } \\
\text { problems. }\end{array}$ & $\begin{array}{l}\text { Not at } \\
\text { all true }\end{array}$ & $\begin{array}{c}\text { A little } \\
\text { true }\end{array}$ & $\begin{array}{c}\text { Pretty } \\
\text { much true }\end{array}$ & $\begin{array}{l}\text { Very much } \\
\text { true }\end{array}$ \\
\hline
\end{tabular}


Social-Emotional Health Survey-Secondary (SEHS-S): Grades 7-12 (Page 2/2)

\begin{tabular}{|c|c|c|c|c|c|}
\hline \multicolumn{2}{|c|}{...Continued } & \multirow{2}{*}{$\begin{array}{c}\begin{array}{c}\text { Not at } \\
\text { all true }\end{array} \\
\text { Not at } \\
\text { all true }\end{array}$} & \multirow{2}{*}{$\begin{array}{c}\begin{array}{c}\text { A little } \\
\text { true }\end{array} \\
\text { A little } \\
\text { true }\end{array}$} & \multirow{2}{*}{$\begin{array}{c}\begin{array}{c}\text { Pretty } \\
\text { much true }\end{array} \\
\begin{array}{c}\text { Pretty } \\
\text { much true }\end{array}\end{array}$} & \multirow{2}{*}{$\begin{array}{c}\begin{array}{c}\text { Very } \\
\text { much true }\end{array} \\
\text { Very much } \\
\text { true }\end{array}$} \\
\hline 18 & $\begin{array}{l}\text { I have a friend my age who helps me when I'm having a } \\
\text { hard time. }\end{array}$ & & & & \\
\hline 19 & I accept responsibility for my actions. & $\begin{array}{l}\text { Not at } \\
\text { all true }\end{array}$ & $\begin{array}{c}\text { A little } \\
\text { true }\end{array}$ & $\begin{array}{c}\text { Pretty } \\
\text { much true }\end{array}$ & $\begin{array}{l}\text { Very much } \\
\text { true }\end{array}$ \\
\hline 20 & When I make a mistake, I admit it. & $\begin{array}{l}\text { Not at } \\
\text { all true }\end{array}$ & $\begin{array}{c}\text { A little } \\
\text { true }\end{array}$ & $\begin{array}{c}\text { Pretty } \\
\text { much true }\end{array}$ & $\begin{array}{l}\text { Very much } \\
\text { true }\end{array}$ \\
\hline 21 & I can deal with being told no. & $\begin{array}{l}\text { Not at } \\
\text { all true }\end{array}$ & $\begin{array}{c}\text { A little } \\
\text { true }\end{array}$ & $\begin{array}{c}\text { Pretty } \\
\text { much true }\end{array}$ & $\begin{array}{l}\text { Very much } \\
\text { true }\end{array}$ \\
\hline 22 & I feel bad when someone gets his or her feelings hurt. & $\begin{array}{l}\text { Not at } \\
\text { all true }\end{array}$ & $\begin{array}{l}\text { A little } \\
\text { true }\end{array}$ & $\begin{array}{c}\text { Pretty } \\
\text { much true }\end{array}$ & $\begin{array}{l}\text { Very much } \\
\text { true }\end{array}$ \\
\hline 23 & I try to understand what other people go through. & $\begin{array}{l}\text { Not at } \\
\text { all true }\end{array}$ & $\begin{array}{c}\text { A little } \\
\text { true }\end{array}$ & $\begin{array}{c}\text { Pretty } \\
\text { much true }\end{array}$ & $\begin{array}{l}\text { Very much } \\
\text { true }\end{array}$ \\
\hline 24 & I try to understand how other people feel and think. & $\begin{array}{l}\text { Not at } \\
\text { all true }\end{array}$ & $\begin{array}{c}\text { A little } \\
\text { true }\end{array}$ & $\begin{array}{c}\text { Pretty } \\
\text { much true }\end{array}$ & $\begin{array}{l}\text { Very much } \\
\text { true }\end{array}$ \\
\hline 25 & I can wait for what I want. & $\begin{array}{l}\text { Not at } \\
\text { all true }\end{array}$ & $\begin{array}{l}\text { A little } \\
\text { true }\end{array}$ & $\begin{array}{c}\text { Pretty } \\
\text { much true }\end{array}$ & $\begin{array}{l}\text { Very much } \\
\text { true }\end{array}$ \\
\hline 26 & I don't bother others when they are busy. & $\begin{array}{l}\text { Not at } \\
\text { all true }\end{array}$ & $\begin{array}{c}\text { A little } \\
\text { true }\end{array}$ & $\begin{array}{c}\text { Pretty } \\
\text { much true }\end{array}$ & $\begin{array}{l}\text { Very much } \\
\text { true }\end{array}$ \\
\hline 27 & I think before I act. & $\begin{array}{l}\text { Not at } \\
\text { all true }\end{array}$ & $\begin{array}{c}\text { A little } \\
\text { true }\end{array}$ & $\begin{array}{c}\text { Pretty } \\
\text { much true }\end{array}$ & $\begin{array}{l}\text { Very much } \\
\text { true }\end{array}$ \\
\hline 28 & Each day I look forward to having a lot of fun. & $\begin{array}{l}\text { Not at } \\
\text { all true }\end{array}$ & $\begin{array}{c}\text { A little } \\
\text { true }\end{array}$ & $\begin{array}{c}\text { Pretty } \\
\text { much true }\end{array}$ & $\begin{array}{l}\text { Very much } \\
\text { true }\end{array}$ \\
\hline 29 & $\begin{array}{l}\text { Overall, I expect more good things to happen to me than } \\
\text { bad things. }\end{array}$ & $\begin{array}{l}\text { Not at } \\
\text { all true }\end{array}$ & $\begin{array}{c}\text { A little } \\
\text { true }\end{array}$ & $\begin{array}{c}\text { Pretty } \\
\text { much true }\end{array}$ & $\begin{array}{l}\text { Very much } \\
\text { true }\end{array}$ \\
\hline 30 & I usually expect to have a good day. & $\begin{array}{l}\text { Not at } \\
\text { all true }\end{array}$ & $\begin{array}{c}\text { A little } \\
\text { true }\end{array}$ & $\begin{array}{c}\text { Pretty } \\
\text { much true }\end{array}$ & $\begin{array}{l}\text { Very much } \\
\text { true }\end{array}$ \\
\hline 31 & On most days I feel energetic. & $\begin{array}{l}\text { Not at } \\
\text { all true }\end{array}$ & $\begin{array}{c}\text { A little } \\
\text { true }\end{array}$ & $\begin{array}{c}\text { Pretty } \\
\text { much true }\end{array}$ & $\begin{array}{l}\text { Very much } \\
\text { true }\end{array}$ \\
\hline 32 & On most days I feel active. & $\begin{array}{l}\text { Not at } \\
\text { all true }\end{array}$ & $\begin{array}{c}\text { A little } \\
\text { true }\end{array}$ & $\begin{array}{c}\text { Pretty } \\
\text { much true }\end{array}$ & $\begin{array}{l}\text { Very much } \\
\text { true }\end{array}$ \\
\hline 33 & On most days I feel enthusiastic. & $\begin{array}{l}\text { Not at } \\
\text { all true }\end{array}$ & $\begin{array}{c}\text { A little } \\
\text { true }\end{array}$ & $\begin{array}{c}\text { Pretty } \\
\text { much true }\end{array}$ & $\begin{array}{l}\text { Very much } \\
\text { true }\end{array}$ \\
\hline 34 & On most days I feel, grateful. & $\begin{array}{l}\text { Not at } \\
\text { all true }\end{array}$ & $\begin{array}{c}\text { A little } \\
\text { true }\end{array}$ & $\begin{array}{c}\text { Pretty } \\
\text { much true }\end{array}$ & $\begin{array}{l}\text { Very much } \\
\text { true }\end{array}$ \\
\hline 35 & On most days I feel, thankful. & $\begin{array}{l}\text { Not at } \\
\text { all true }\end{array}$ & $\begin{array}{c}\text { A little } \\
\text { true }\end{array}$ & $\begin{array}{c}\text { Pretty } \\
\text { much true }\end{array}$ & $\begin{array}{l}\text { Very much } \\
\text { true }\end{array}$ \\
\hline 36 & On most days I feel, appreciative. & $\begin{array}{l}\text { Not at } \\
\text { all true }\end{array}$ & $\begin{array}{l}\text { A little } \\
\text { true }\end{array}$ & $\begin{array}{c}\text { Pretty } \\
\text { much true }\end{array}$ & $\begin{array}{l}\text { Very much } \\
\text { true }\end{array}$ \\
\hline
\end{tabular}

Copyright: Michael J. Furlong, UCSB Project CoVitality. We support use of the SEHS-Secondary to foster youths' positive social emotional health. Please contact us: $\underline{\text { mfurlong@ucsb.edu }}$ 


\section{Social-Emotional Health Survey-Secondary (SEHS-S): Scoring Form (Page 1/2)}

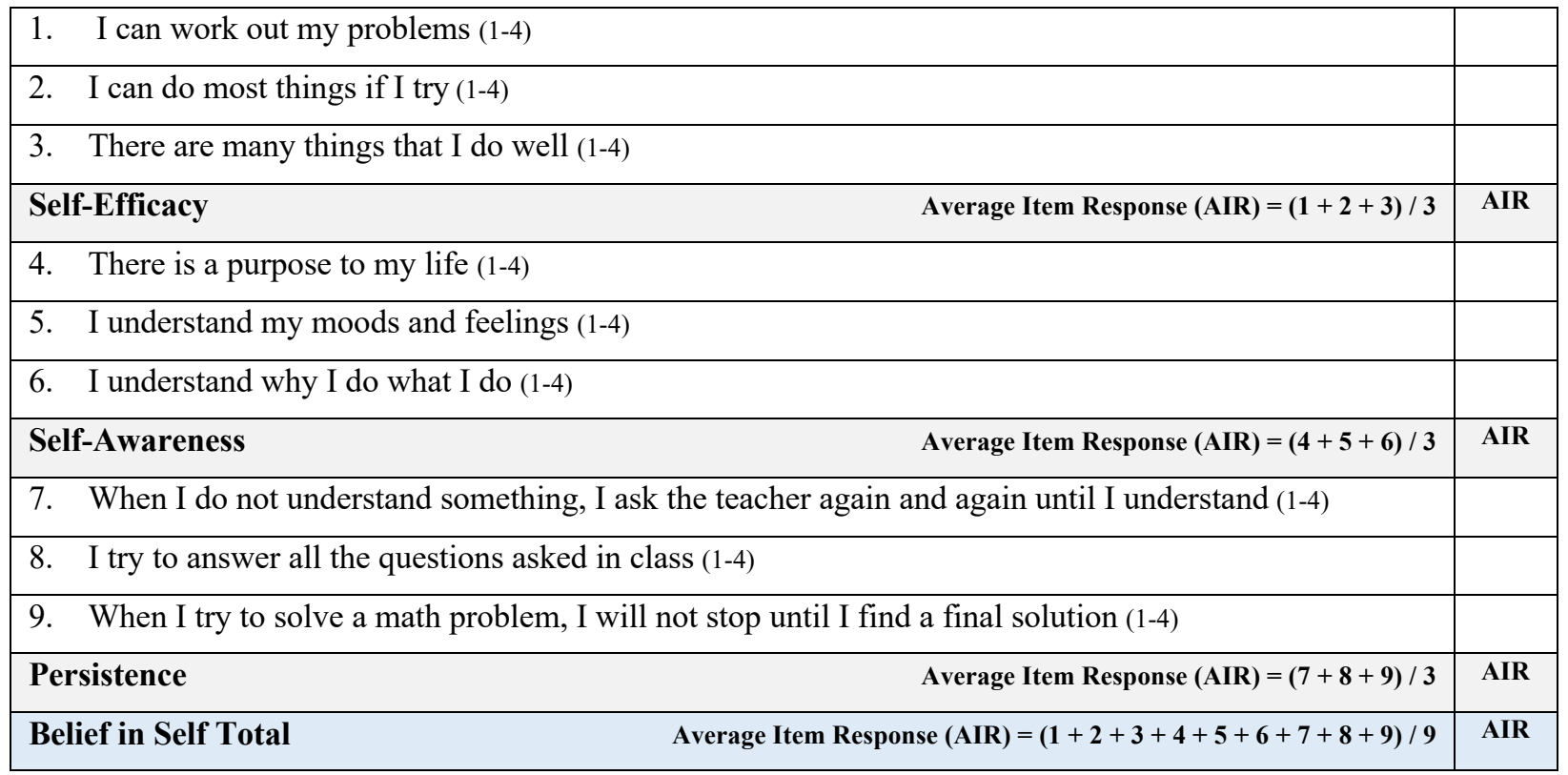

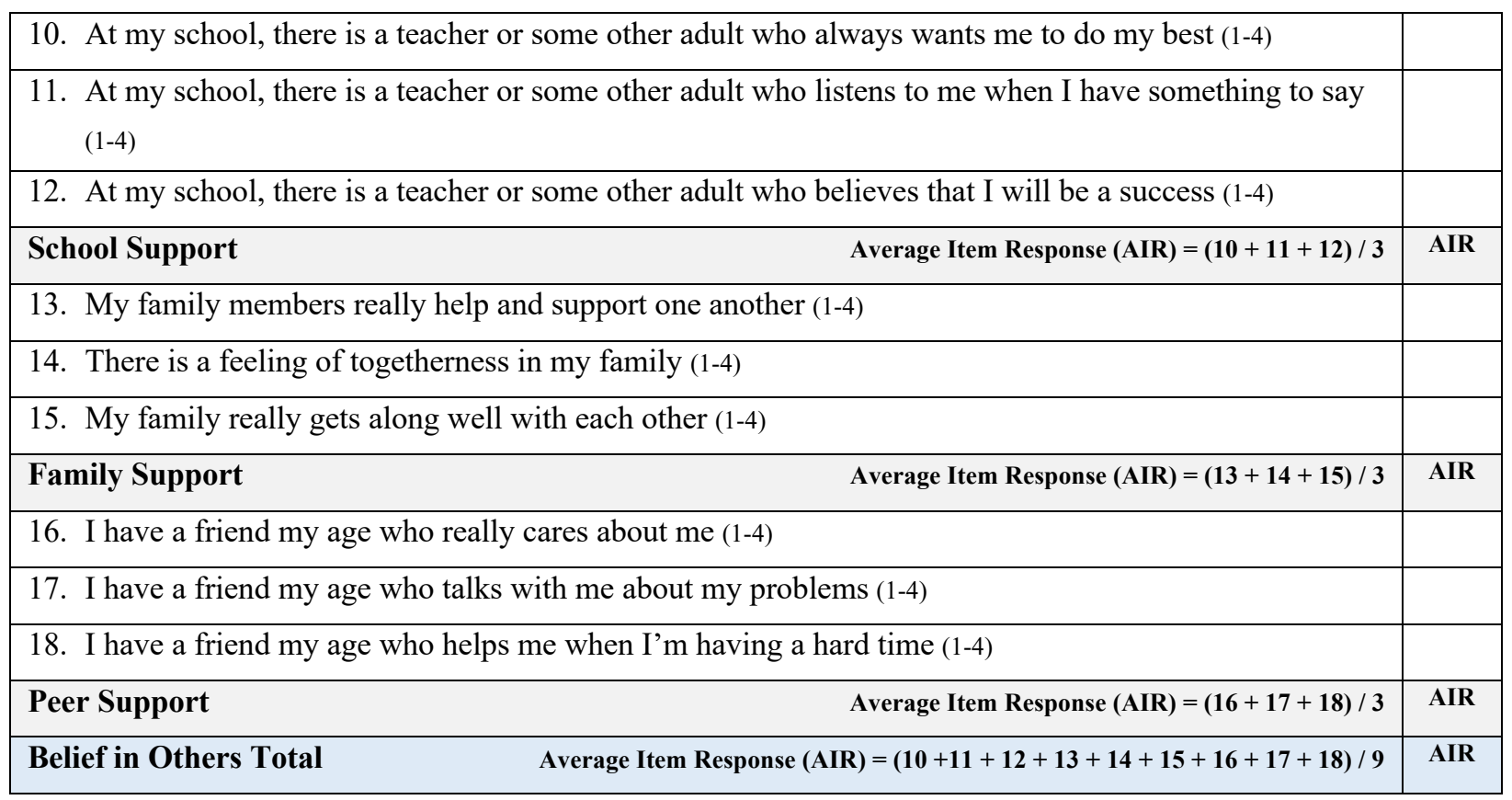




\section{Social-Emotional Health Survey-Secondary (SEHS-S): Scoring Form (Page 2/2)}

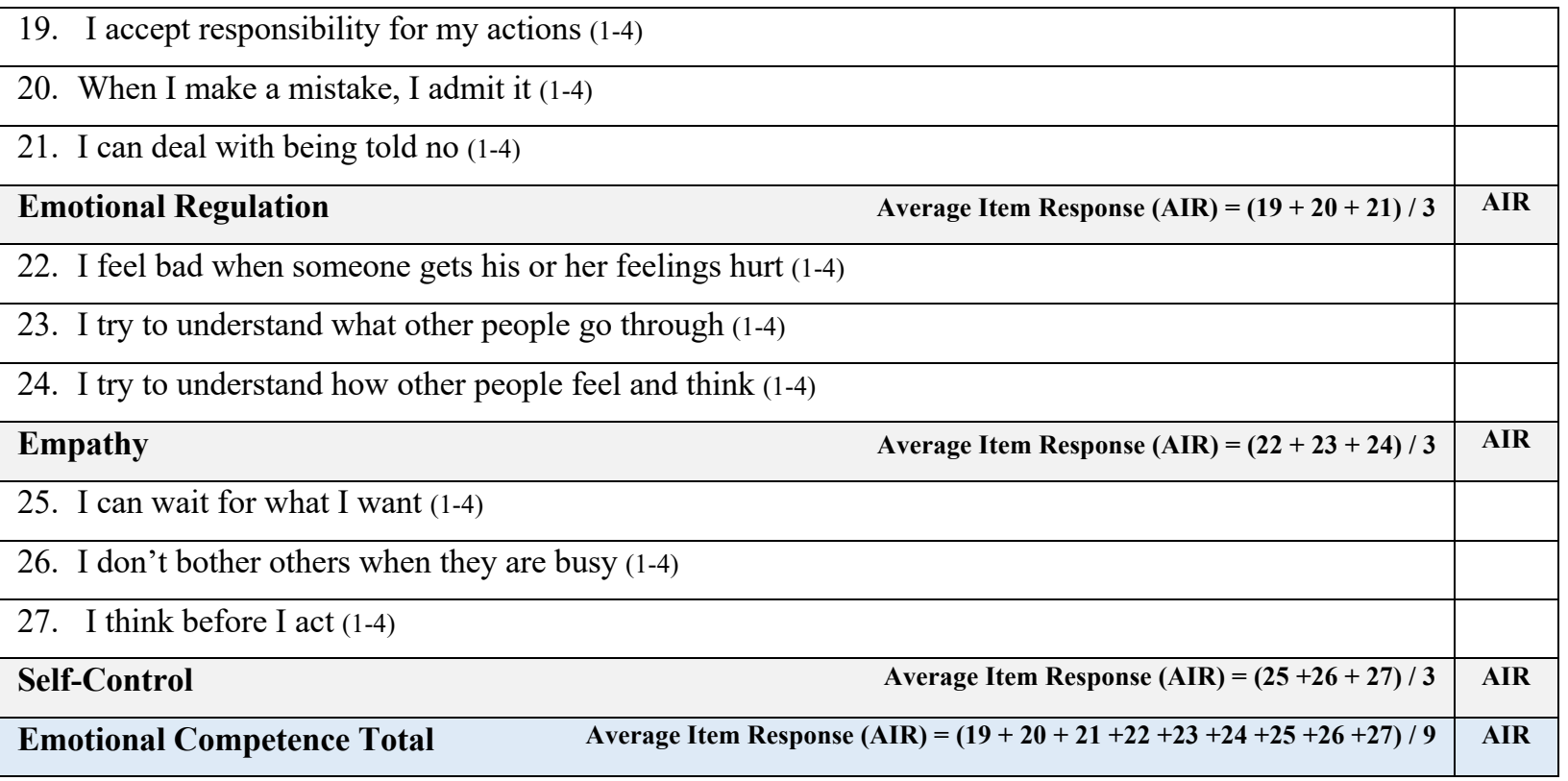

\begin{tabular}{|c|c|c|}
\hline \multicolumn{3}{|c|}{ 28. Each day I look forward to having a lot of fun (1-4) } \\
\hline 29. I usually expect to & $\mathrm{y}(1-4)$ & \\
\hline \multicolumn{3}{|c|}{ 30. Overall, I expect more good things to happen to me than bad things (1-4) } \\
\hline Optimism & Average Item Response (AIR) $=(28+29+30) / 3$ & AIR \\
\hline \multicolumn{3}{|c|}{ 31. Since yesterday how much have you felt grateful (1-4) } \\
\hline \multicolumn{3}{|c|}{ 32. Since yesterday how much have you felt thankful. (1-4) } \\
\hline \multicolumn{3}{|c|}{ 33. Since yesterday how much have you felt appreciative. (1-4) } \\
\hline Gratitude & Average Item Response $(\mathrm{AIR})=(31+32+33) / 3$ & AIR \\
\hline \multicolumn{3}{|c|}{ 34. How much do you feel energetic right now (1-4) } \\
\hline \multicolumn{3}{|c|}{ 35. How much do you feel active right now (1-4) } \\
\hline \multicolumn{3}{|c|}{ 36. How much do you feel enthusiastic right now (1-4) } \\
\hline Zest & Average Item Response (AIR) $=(34+35+36) / 3$ & AIR \\
\hline Engaged Living Total & Average Item Response (AIR) $=(28+29+30+31+32+33+34+35+36) / 9$ & AIR \\
\hline
\end{tabular}

\begin{tabular}{|l|c|}
\hline Summary Average Item Responses (AIR) & (transfer values from above here) \\
\hline Belief in Self (BIS) & AIR \\
\hline Belief in Others (BIO) & AIR \\
\hline Emotional Competence (EC) & AIR \\
\hline Engaged Living (EL) Average Item Response (AIR) $=$ (BIS + BIO + EC + EL)/4 & AIR \\
\hline Total Covitality
\end{tabular}

Copyright: Michael J. Furlong, UCSB Project CoVitality. We support use of the SEHS-S to foster youths' positive social emotional health. Please contact us: $\underline{\text { murlong@ucsb.edu }}$ 


\begin{tabular}{|c|c|c|c|c|c|c|c|c|c|c|c|c|c|c|c|c|c|}
\hline AIR & S-E & S-A & Per & BIS & PS & SS & FS & BIO & $\mathrm{E}$ & ER & S-C & EC & $\mathrm{O}$ & $\mathrm{G}$ & Z & EL & $\mathrm{CoV}$ \\
\hline 4.0 & & & & 4.0 & & & & 4.0 & & & & 4.0 & & & & 4.0 & 4.0 \\
\hline 3.9 & & & & & & & & & & & & & & & & & 3.9 \\
\hline 3.8 & & & & & & & & & & & & & & & & & 3.8 \\
\hline 3.7 & & & & & & & & & & & & & & & & & 3.7 \\
\hline 3.6 & & & & & & & & & & & & & & & & & 3.6 \\
\hline 3.5 & & & & & & & & & & & & & & & & & 3.5 \\
\hline 3.4 & & & & & & & & & & & & & & & & & 3.4 \\
\hline 3.3 & & & & & & & & & & & & & & & & & 3.3 \\
\hline 3.2 & & & & & & & & & & & & & & & & & 3.2 \\
\hline 3.1 & & & & & & & & & & & & & & & & & 3.1 \\
\hline 3.0 & & & & 3.0 & & & & 3.0 & & & & 3.0 & & & & 3.0 & 3.0 \\
\hline 2.9 & & & & & & & & & & & & & & & & & 2.9 \\
\hline 2.8 & & & & & & & & & & & & & & & & & 2.8 \\
\hline 2.7 & & & & & & & & & & & & & & & & & 2.7 \\
\hline 2.6 & & & & & & & & & & & & & & & & & 2.6 \\
\hline 2.5 & & & & & & & & & & & & & & & & & 2.5 \\
\hline 2.4 & & & & & & & & & & & & & & & & & 2.4 \\
\hline 2.3 & & & & & & & & & & & & & & & & & 2.3 \\
\hline 2.2 & & & & & & & & & & & & & & & & & 2.2 \\
\hline 2.1 & & & & & & & & & & & & & & & & & 2.1 \\
\hline 2.0 & & & & 2.0 & & & & 2.0 & & & & 2.0 & & & & 2.0 & 2.0 \\
\hline 1.9 & & & & & & & & & & & & & & & & & 1.9 \\
\hline 1.8 & & & & & & & & & & & & & & & & & 1.8 \\
\hline 1.7 & & & & & & & & & & & & & & & & & 1.7 \\
\hline 1.6 & & & & & & & & & & & & & & & & & 1.6 \\
\hline 1.5 & & & & & & & & & & & & & & & & & 1.5 \\
\hline 1.4 & & & & & & & & & & & & & & & & & 1.4 \\
\hline 1.3 & & & & & & & & & & & & & & & & & 1.3 \\
\hline 1.2 & & & & & & & & & & & & & & & & & 1.2 \\
\hline 1.1 & & & & & & & & & & & & & & & & & 1.1 \\
\hline 1.0 & & & & 1.0 & & & & 1.0 & & & & 1.0 & & & & 1.0 & 1.0 \\
\hline & 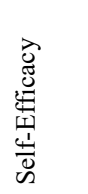 & 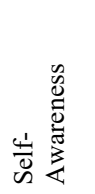 & 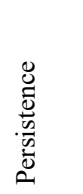 & 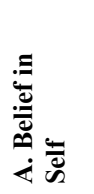 & 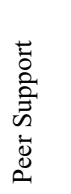 & 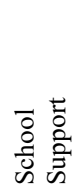 & 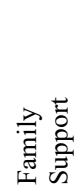 & 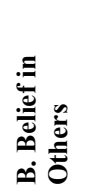 & 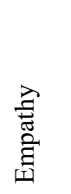 & 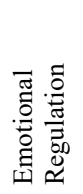 & 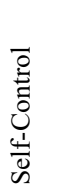 & 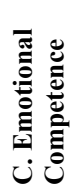 & 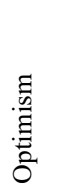 & 葋 & 芯 & 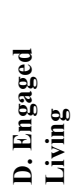 & 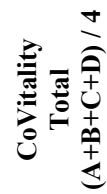 \\
\hline
\end{tabular}

Responses: $1=$ not at all true, $2=$ a little true, $3=$ pretty much true, $4=$ very much true. $\quad$ CoV $=\mathrm{AIR}$ of $(\mathrm{A}+\mathrm{B}+\mathrm{C}+\mathrm{D}) / 4=$

Shaded $=\mathrm{AIR} \pm$ standard deviation (16th to 84th percentiles). Based on the responses of 119, 756 California students in Grades 7-12.

Note. Copyright: Michael J. Furlong, UCSB Project CoVitality. We support use of the SEHS-S to foster youths' positive social emotional health. Please contact us: mfurlong@ $@$ ucsb.edu 


\section{Appendix C}

\section{Social-Emotional Distress Scale-Secondary (SEDS-S): Grades 7-12 (Page 1/1)}

Directions: You are being asked to take a survey about how you have felt over the past few weeks. Your school is doing this survey to better understand your school experiences. With this information, your school wants to provide support to help improve your school experiences.

\begin{tabular}{|c|l|c|c|c|c|}
\hline \multicolumn{2}{|l|}{$\begin{array}{l}\text { Read each item and choose the response that best describes you. } \\
\text { Please respond honestly. There are no right or wrong answers. } \\
\text { You can skip questions you don't want to answer. }\end{array}$} & $\begin{array}{c}\text { Not at } \\
\text { all true }\end{array}$ & $\begin{array}{c}\text { A little } \\
\text { true }\end{array}$ & $\begin{array}{c}\text { Pretty } \\
\text { much true }\end{array}$ & $\begin{array}{c}\text { Very } \\
\text { much } \\
\text { true }\end{array}$ \\
\hline 1 & I had a hard time breathing because I was anxious (1-4) & $\begin{array}{c}\text { Not at } \\
\text { all true }\end{array}$ & $\begin{array}{c}\text { A little } \\
\text { true }\end{array}$ & $\begin{array}{c}\text { Pretty } \\
\text { much true }\end{array}$ & $\begin{array}{c}\text { Very } \\
\text { much true }\end{array}$ \\
\hline 2 & I worried that I would embarrass myself in front of others (1-4) & $\begin{array}{c}\text { Not at } \\
\text { all true }\end{array}$ & $\begin{array}{c}\text { A little } \\
\text { true }\end{array}$ & $\begin{array}{c}\text { Pretty } \\
\text { much true }\end{array}$ & $\begin{array}{c}\text { Very } \\
\text { much true }\end{array}$ \\
\hline 3 & I was tense and uptight (1-4) & $\begin{array}{c}\text { Not at } \\
\text { all true }\end{array}$ & $\begin{array}{c}\text { A little } \\
\text { true }\end{array}$ & $\begin{array}{c}\text { Pretty } \\
\text { much true }\end{array}$ & $\begin{array}{c}\text { Very } \\
\text { much true }\end{array}$ \\
\hline 4 & I had a hard time relaxing (1-4) & $\begin{array}{c}\text { Not at } \\
\text { all true }\end{array}$ & $\begin{array}{c}\text { A little } \\
\text { true }\end{array}$ & $\begin{array}{c}\text { Pretty } \\
\text { much true }\end{array}$ & $\begin{array}{c}\text { Very } \\
\text { much true }\end{array}$ \\
\hline 5 & I felt sad and down (1-4) & $\begin{array}{c}\text { Not at } \\
\text { all true }\end{array}$ & $\begin{array}{c}\text { A little } \\
\text { true }\end{array}$ & $\begin{array}{c}\text { Pretty } \\
\text { much true }\end{array}$ & $\begin{array}{c}\text { Very } \\
\text { much true }\end{array}$ \\
\hline 6 & I was easily irritated (1-4) & $\begin{array}{c}\text { Not at } \\
\text { all true }\end{array}$ & $\begin{array}{c}\text { A little } \\
\text { true }\end{array}$ & $\begin{array}{c}\text { Pretty } \\
\text { much true }\end{array}$ & $\begin{array}{c}\text { Very } \\
\text { much true }\end{array}$ \\
\hline 7 & It was hard for me to get excited about anything (1-4) & $\begin{array}{c}\text { Not at } \\
\text { all true }\end{array}$ & $\begin{array}{c}\text { A little } \\
\text { true }\end{array}$ & $\begin{array}{c}\text { Pretty } \\
\text { much true }\end{array}$ & $\begin{array}{c}\text { Very } \\
\text { much true }\end{array}$ \\
\hline 8 & I was easily annoyed and sensitive (1-4) & $\begin{array}{c}\text { Not at } \\
\text { all true }\end{array}$ & $\begin{array}{c}\text { A little } \\
\text { true }\end{array}$ & $\begin{array}{c}\text { Pretty } \\
\text { much true }\end{array}$ & $\begin{array}{c}\text { Very } \\
\text { much true }\end{array}$ \\
\hline 9 & I was scared for no good reason (1-4) & $\begin{array}{c}\text { Not at } \\
\text { all true }\end{array}$ & $\begin{array}{c}\text { A little } \\
\text { true }\end{array}$ & $\begin{array}{c}\text { Pretty } \\
\text { much true }\end{array}$ & $\begin{array}{c}\text { Very } \\
\text { much true }\end{array}$ \\
\hline 10 & It was hard for me to cope and I thought I would panic (1-4) & $\begin{array}{c}\text { Not at } \\
\text { all true }\end{array}$ & $\begin{array}{c}\text { A little } \\
\text { true }\end{array}$ & $\begin{array}{c}\text { Pretty } \\
\text { much true }\end{array}$ & $\begin{array}{c}\text { Very } \\
\text { much true }\end{array}$ \\
\hline
\end{tabular}

Copyright: Michael J. Furlong, UCSB Project CoVitality. We support use of the SEDS-S to foster youths' positive social emotional health. Please contact us: $\underline{\text { murlong@ucsb.edu }}$ 


\section{Social-Emotional Distress Scale-Secondary (SEDS-S): Scoring Form}

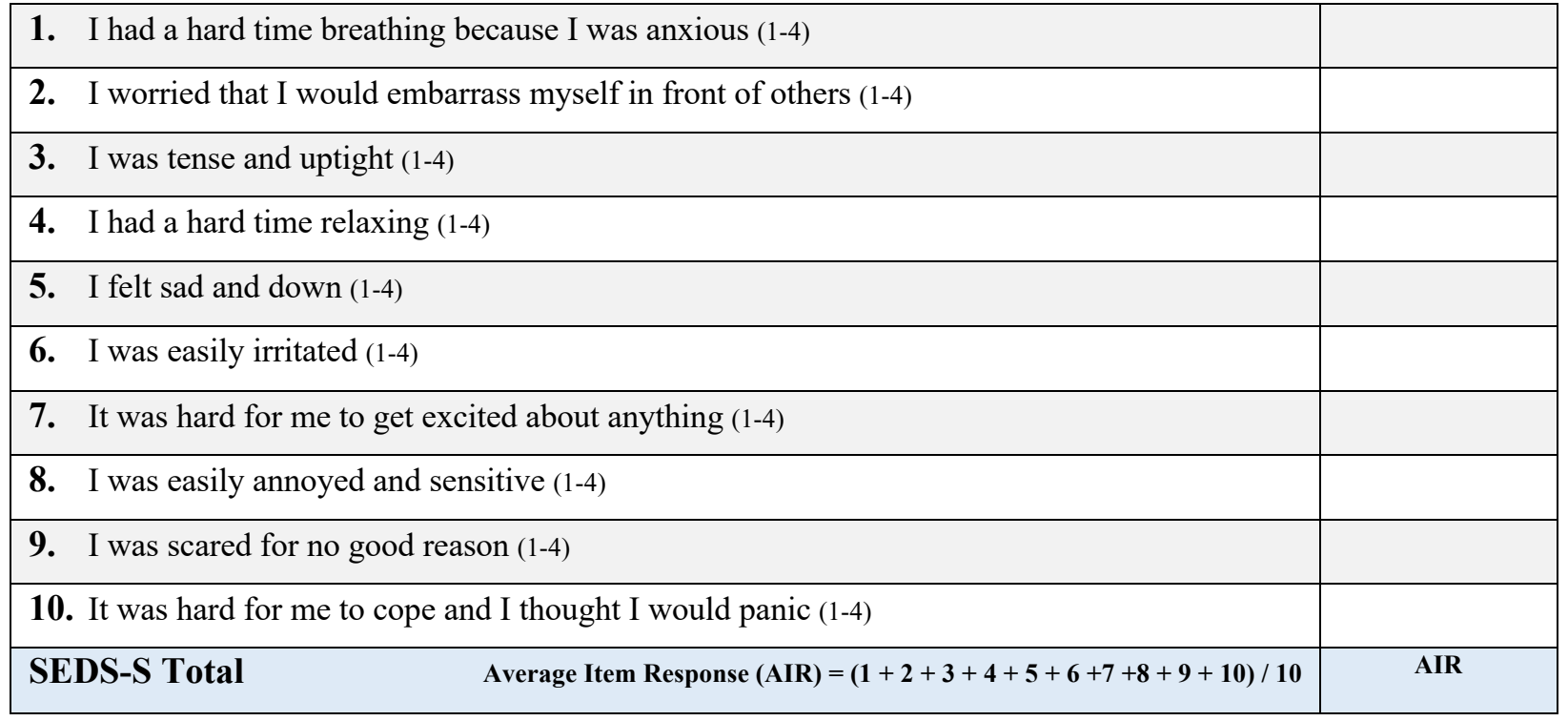

\section{Social-Emotional Distress Scale-Secondary (SEDS-S): Scoring Form}

\begin{tabular}{|c|c|c|c|c|c|c|c|c|c|c|}
\hline Item Response & 1 & 2 & 3 & 4 & 5 & 6 & 7 & 8 & 9 & 10 \\
\hline \multicolumn{11}{|l|}{ Very much true (4) } \\
\hline \multicolumn{11}{|c|}{ Pretty much true (3) } \\
\hline \multicolumn{11}{|l|}{ A little true (2) } \\
\hline \multicolumn{11}{|l|}{ Not at all true (1) } \\
\hline & 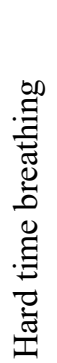 & 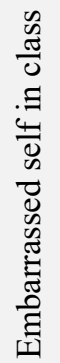 & $\begin{array}{l}\vec{E} \\
.00 \\
\stackrel{0}{0} \\
\Xi \\
0 \\
0 \\
0 \\
0 \\
0\end{array}$ & 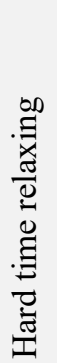 & 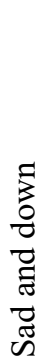 & 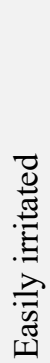 & 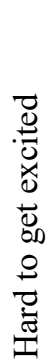 & 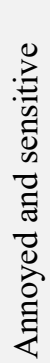 & 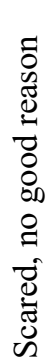 & 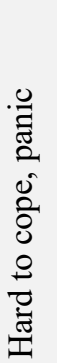 \\
\hline
\end{tabular}

Responses: $\mathrm{AIR}=2.0 \ldots$ standard deviation $=1.0 \ldots 16$ th to 84 th percentile range $=1.0$ to $3.0 \ldots$ AIR based on responses of 119,756 California students in grades 7-12. For access to prevention and intervention strategy resources, see http://project-covitality.info/prevention-and-intervention/ 


\section{Appendix D}

Systematic Review of SRB: Overview of Critical Findings

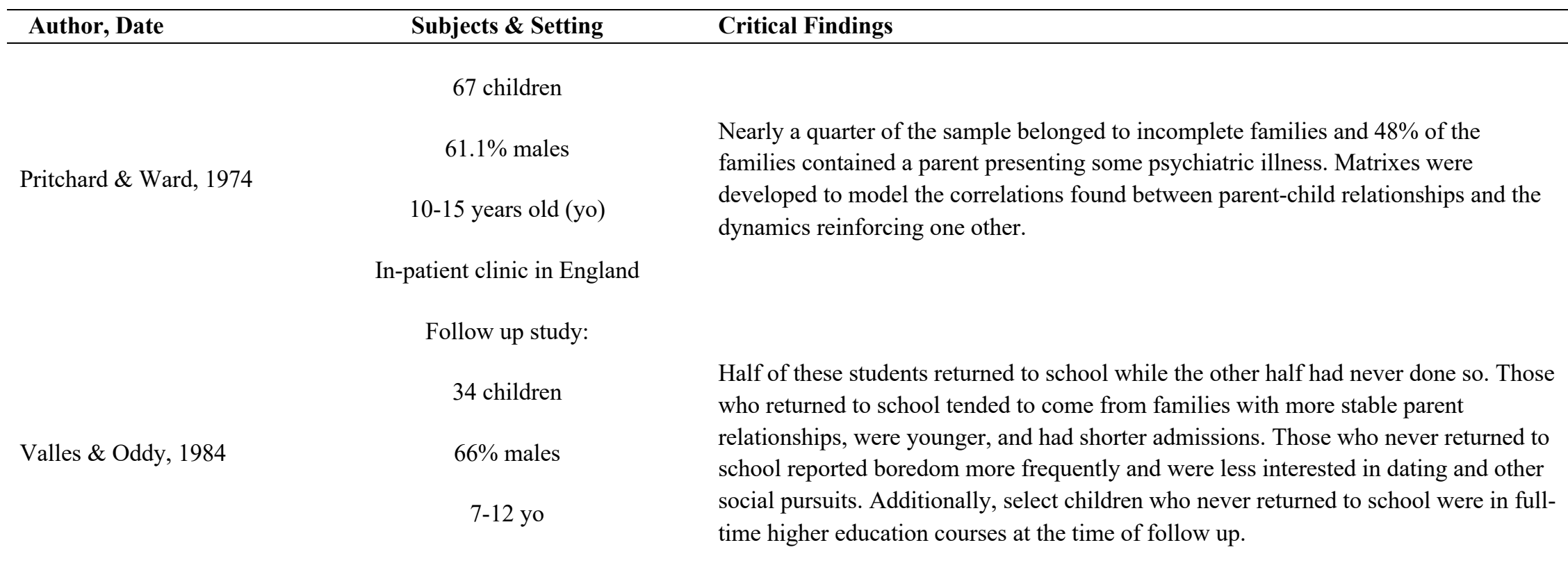

In-patient clinic

76 families

Bernstein, Svingen, \&

$55 \%$ males

Garfinkel, 1990

$7-17$ yo

Outpatient clinic

Significantly less family dysfunction was rated by mothers and children if the child had a diagnosis of pure anxiety disorder compared to families of school phobic children in other diagnostic categories Dyadic dysfunction was identified in the parent-child relationship in families with a school phobic child. Diagnosis was the strongest predictor of family dysfunction, including disruptive behavioral disorders.

Bools, Foster, Brown, \&

100 families

Children from a non-clinical population were divided into groups sharing similar Berg, 1990

$59 \%$ males disturbances. Associations were found between antisocial behaviors/conduct disorder 


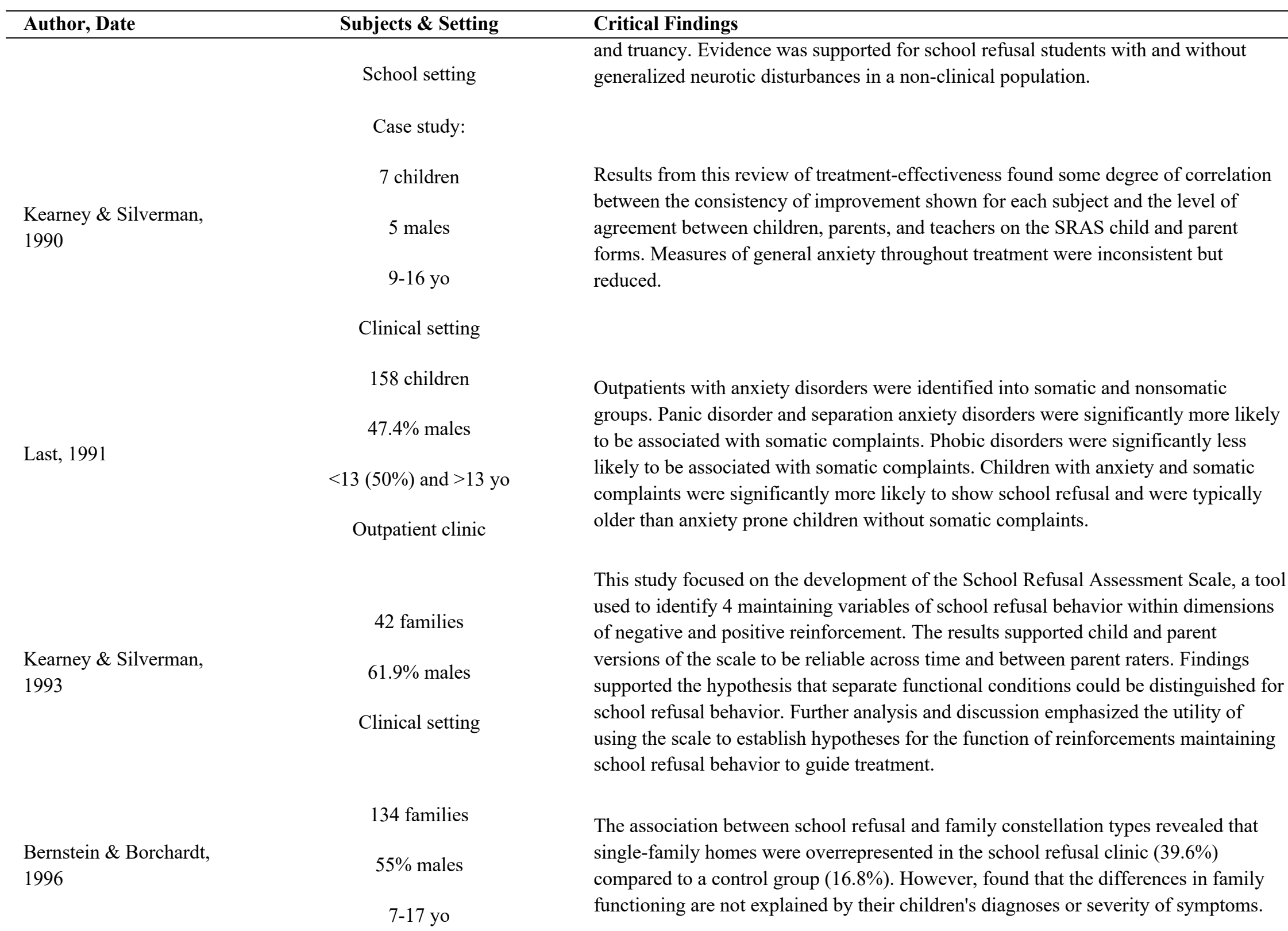




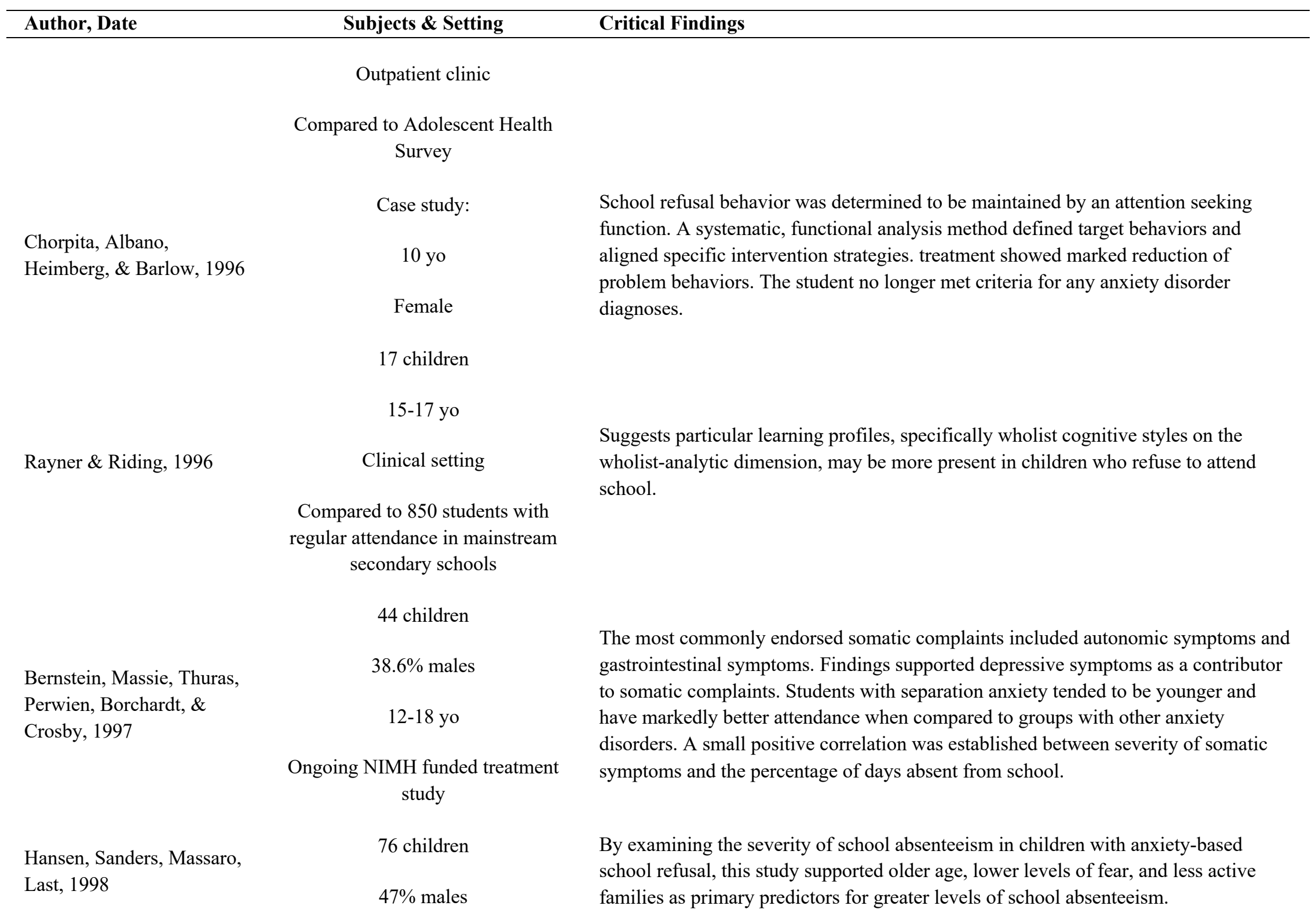




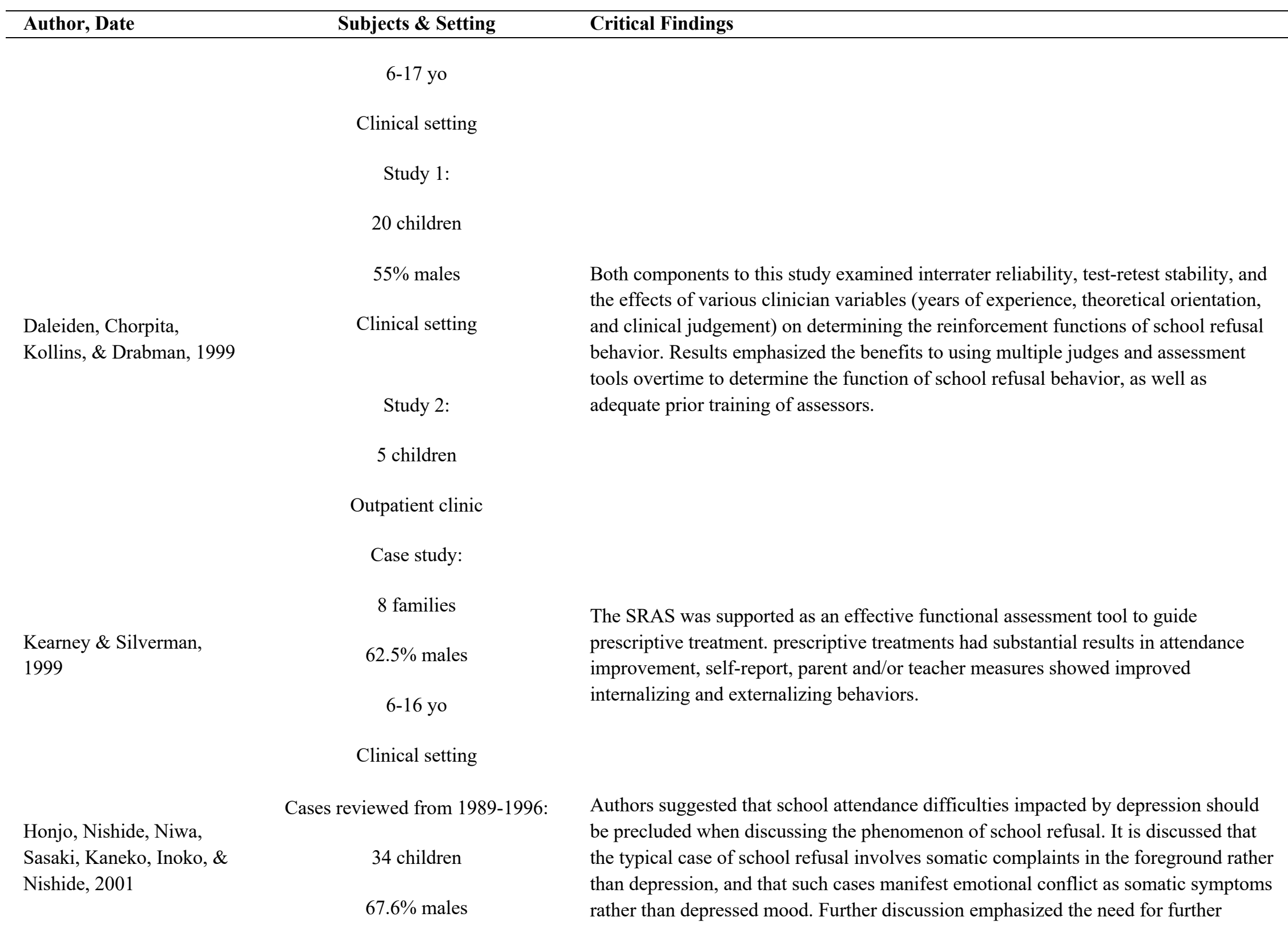




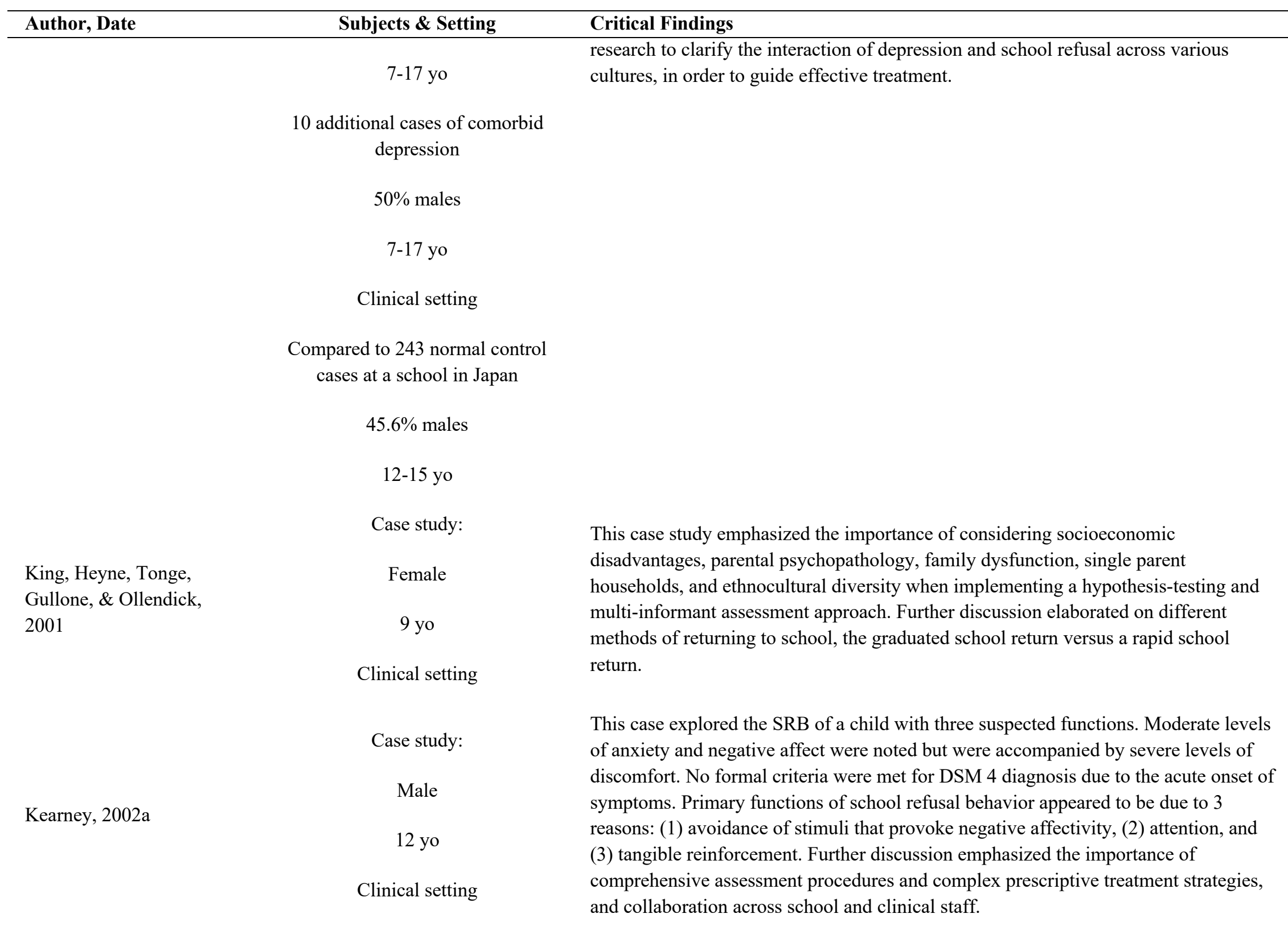




\begin{tabular}{|c|c|c|}
\hline Author, Date & Subjects \& Setting & Critical Findings \\
\hline \multirow[b]{2}{*}{$\begin{array}{l}\text { Egger, Costello, \& Angold, } \\
2003\end{array}$} & 1,422 families & \\
\hline & $\begin{array}{l}\text { From the longitudinal Great Smoky } \\
\text { Mountains Study (GSMS) of the } \\
\text { development of psychiatric disorders } \\
\text { in youths in North Carolina } \\
\text { The original GSMS sample included } \\
55.6 \% \text { males }\end{array}$ & $\begin{array}{l}\text { The association between anxious school refusal, truancy, and psychiatric disorders } \\
\text { was examined in a community sample using descriptive rather than an etiological } \\
\text { definition of SRB. Pure anxious school refusal was associated with depression and } \\
\text { separation anxiety while pure truancy was associated with oppositional defiant } \\
\text { disorder, conduct disorder, and depression. Of the mixed school refusers (anxious and } \\
\text { truancy), } 88.2 \% \text { had a psychiatric disorder and increased rates of both emotional and } \\
\text { behavioral disorders. Further discussion emphasized the importance of evaluating } \\
\text { children with school attendance problems for psychiatric disorders. }\end{array}$ \\
\hline \multirow{3}{*}{$\begin{array}{l}\text { Honjo, Sasaki, Kaneko, } \\
\text { Tachibana, Murase, Ishii, } \\
\text { Nishide. Nishide, } 2003\end{array}$} & Review of cases from 1998: & \\
\hline & $\begin{array}{l}\qquad 46.1 \% \text { males } \\
\text { First-year junior high to second-year } \\
\text { high school age }\end{array}$ & $\begin{array}{l}\text { Factor analysis of CDI rating scales established } 3 \text { factors: (1) core depression, (2) } \\
\text { feelings of interpersonal maladaptation, and ( } 3 \text { ) self-revulsion. School avoidance and } \\
\text { personality scales were designed and analyzed for this study. Findings discussed the } \\
\text { school avoidance factors were intimately associated with both 'feelings of } \\
\text { interpersonal maladaptation' and 'core depression' from the CDI factors. }\end{array}$ \\
\hline & School in Japan & \\
\hline Kearney \& Albano, 2004 & $\begin{array}{l}143 \text { families } \\
62.9 \% \text { males } \\
5-17 \text { yo } \\
\text { Clinical setting }\end{array}$ & $\begin{array}{l}\text { When examining diagnoses most commonly associated with proposed functions of } \\
\text { school refusal behavior, results indicated significant heterogeneity across this } \\
\text { population. Children tended to be older if they refused school to escape aversive social } \\
\text { and/or evaluative situations or to pursue tangible reinforcement outside of school. } \\
\text { Children tended to have the most severe diagnoses if they refused school to avoid } \\
\text { stimuli that provoked negative affectivity. It was also acknowledged that while some } \\
\text { children may meet criteria for a separation anxiety disorder, their learned behaviors to } \\
\text { evade school may be rooted in willful, manipulative, and controlling behaviors (e.g., } \\
\text { tantrums). This study supported that the sole reliance on formal diagnoses or } \\
\text { taxonomies to organize youth with school refusal behavior is a difficult task. } \\
\text { Considerable heterogeneity is evident and much overlap across proposed diagnostic }\end{array}$ \\
\hline
\end{tabular}


Follow up study:

\section{8 children}

McShane, Walter, \& Rey, 2004

Kearney, Chapman, \& Cook, 2005

Kearney, 2007

Girimaji, \& Seshardi, 2007
12-18 yo

Clinical setting

Previously assessed in 1994-1998

55 young children

5-9 yo

Clinical setting

222 families

$60.3 \%$ males

5-17 yo

Clinical setting

Review of records:

33 children

$57.6 \%$ males
A follow-up with past in- and out-patients sought to inquire about their improved functional outcomes. After six months of treatment, 83 demonstrated general improvements, 89 maintained their functional improvements at 3 years after treatment Dysthymia or comorbid diagnoses were associated with poorer functional outcomes 6 months after treatment, but not at 3 years. Academic difficulties and social phobias in conjunction to SRB had less positive long-term functional outcomes and were predictive of poorer functional outcomes 3 years following treatment.

Children who present SRB are characterized mostly by separation anxiety, attentionseeking motives, and oppositional behavior. Many of these families exhibit low levels of independence or high levels of dependence that may reinforce children's willful, stubborn, manipulative behaviors designed to force parents to indulge their demands. Children with anxiety may continue attending school, but with great distress. Those without anxiety symptoms may refuse school and pursue tangible incentives by demonstrating behaviors that evade clinical diagnoses and are difficult to identify.

This study found the function of school refusal behavior was a better determinant of the degree of school absenteeism than a behavior form. Ongoing analysis of variables that impact treatment effectiveness should be part of evaluations (e.g., problematic family dynamics, demographic variables, developmental status, ethnicity, severity of school refusal behavior, and comorbid mental disorders).

When considering diagnostic status, psychosocial correlates, and short-term outcomes of youth presenting school refusal behavior, $87.9 \%$ of subjects had a psychiatric diagnosis at baseline, depression most common (63.6\%), with specific phobias next (30.3\%); several (48.5\%) presented school related stressors. At follow up, $66.6 \%$ had returned to school, of these, $20 \%$ demonstrated persisting psychiatric diagnoses at a 


\begin{tabular}{|c|c|c|}
\hline Author, Date & Subjects \& Setting & Critical Findings \\
\hline & $\begin{array}{l}\qquad 8-16 \text { yo } \\
\text { Clinical setting in India } \\
\text { Participating in services through } \\
\text { National Institute of Mental Health } \\
\text { and Neuro Sciences (NIMHANS) }\end{array}$ & $\begin{array}{l}\text { follow-up. Results indicated that school refusal had remitted despite their psychiatric } \\
\text { status persisting. }\end{array}$ \\
\hline Arvans \& LeBlanc, 2009 & $\begin{array}{l}\text { Male } \\
14 \text { yo }\end{array}$ & $\begin{array}{l}\text { A young male with Asperger's demonstrating symptoms of depression and anxiety } \\
\text { was found to refuse school in order to escape undesirable settings; additional minor } \\
\text { health problems were also present, including nearly daily reports of migraines. The } \\
\text { authors propose that antecedents to, and the function of migraines may play a vital } \\
\text { role in school refusal behavior problems in select populations. }\end{array}$ \\
\hline \multirow[t]{2}{*}{ Dube \& Orpinas, 2009} & $\begin{array}{l}99 \text { children } \\
58.5 \% \text { males } \\
\text { Upper-elementary to middle school } \\
\text { setting }\end{array}$ & $\begin{array}{l}\text { Social workers examined the reinforcement profiles of students with attendance } \\
\text { problems from SRAS-C reports and identified } 3 \text { profiles: (1) missed to avoid fear- or } \\
\text { anxiety-producing situations, escape from aversive social or evaluative situations, or } \\
\text { gain positive tangible rewards (multiple profile) }(17.2 \%) \text {; (2) missed to gain parental } \\
\text { attention or receive tangible rewards (positive reinforcement) }(60.6 \%) \text {; and ( } 3) \\
\text { students who had no profile }(22.2 \%) \text {. All three groups were found to have } \\
\text { significantly different mean scores for behavioral difficulties. Further discussion } \\
\text { emphasized the importance of developing behavior assessments to identify early signs } \\
\text { of school refusal. }\end{array}$ \\
\hline & 50 children & \\
\hline $\begin{array}{l}\text { Maric, Heyne, de Heus, van } \\
\text { Widenfelt, \& Westenberg, } \\
2012\end{array}$ & $\begin{array}{l}\text { 11-17 yo } \\
\text { Participating in "@ School Project" } \\
\text { program in the Netherlands }\end{array}$ & $\begin{array}{l}\text { Negative cognitions commonly linked to anxiety were analyzed in school refusers } \\
\text { regarding their negative automatic thoughts and thinking errors. When controlling for } \\
\text { anxiety, school refusers were found to report more negative automatic thoughts } \\
\text { concerning personal failure, fewer negative automatic thoughts concerning hostility, } \\
\text { and fewer positive automatic thoughts. Negative automatic thoughts concerning } \\
\text { personal failure and hostility, and the negative cognitive error of overgeneralizing } \\
\text { were found to independently predict school refusal. }\end{array}$ \\
\hline
\end{tabular}




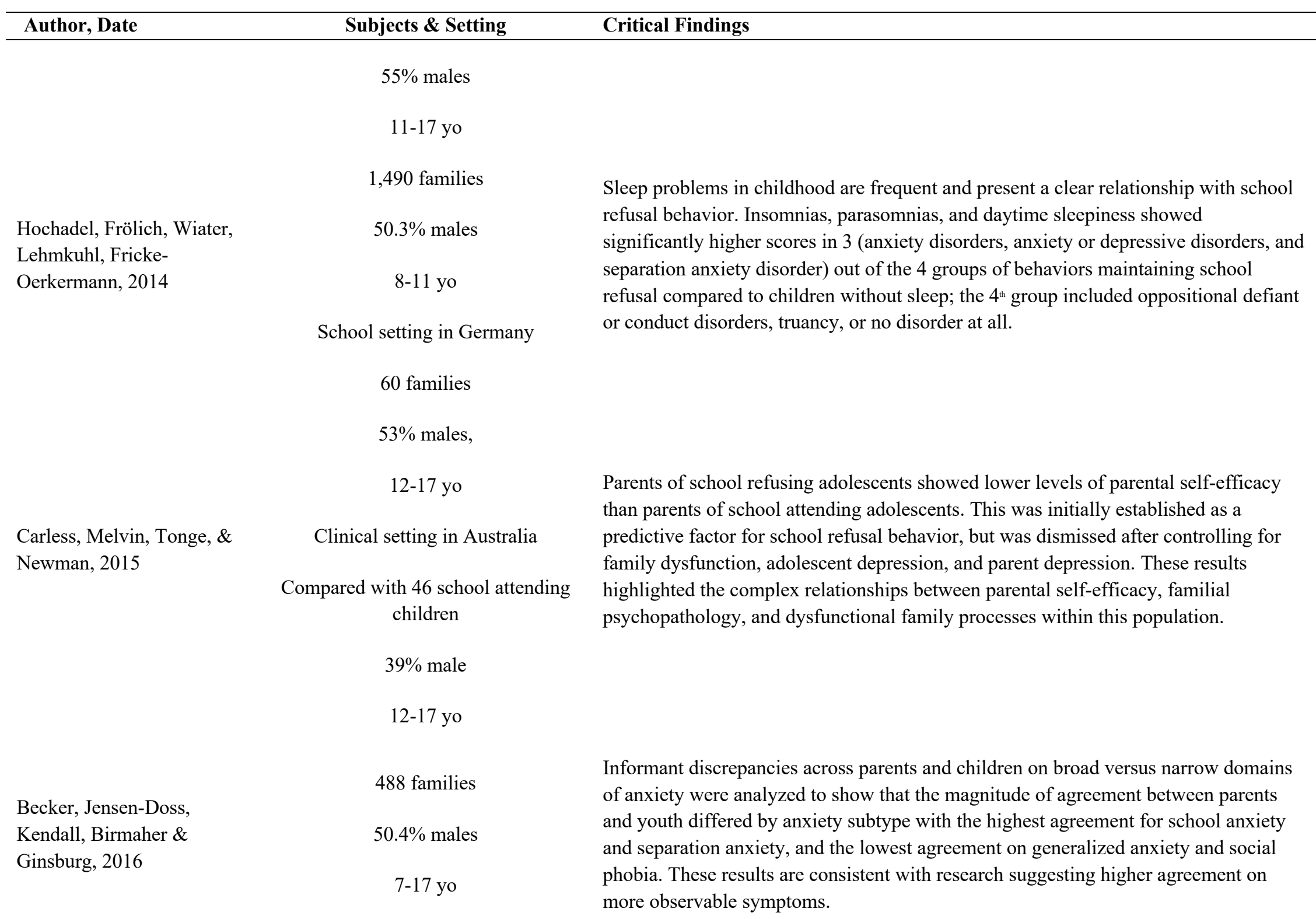




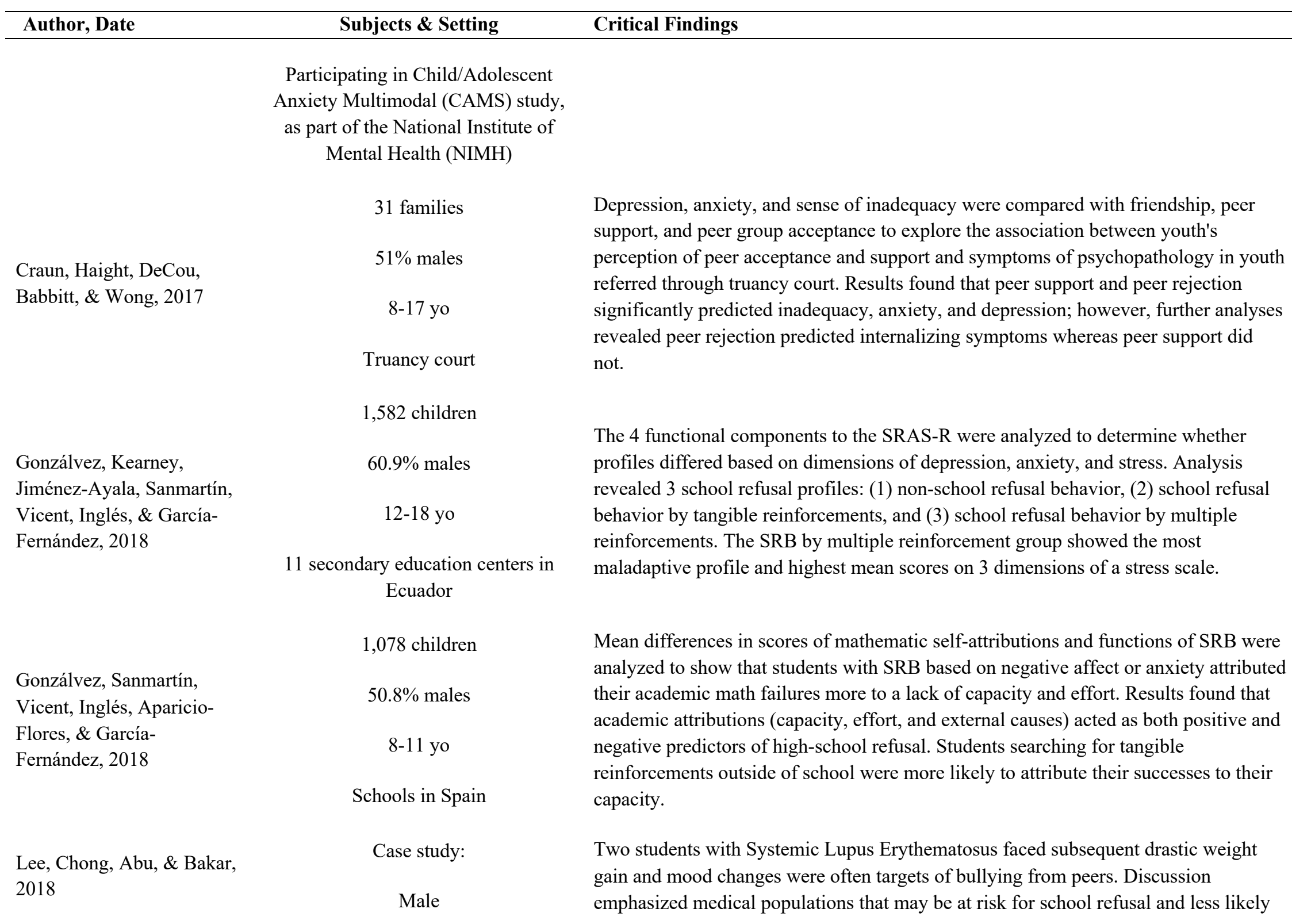




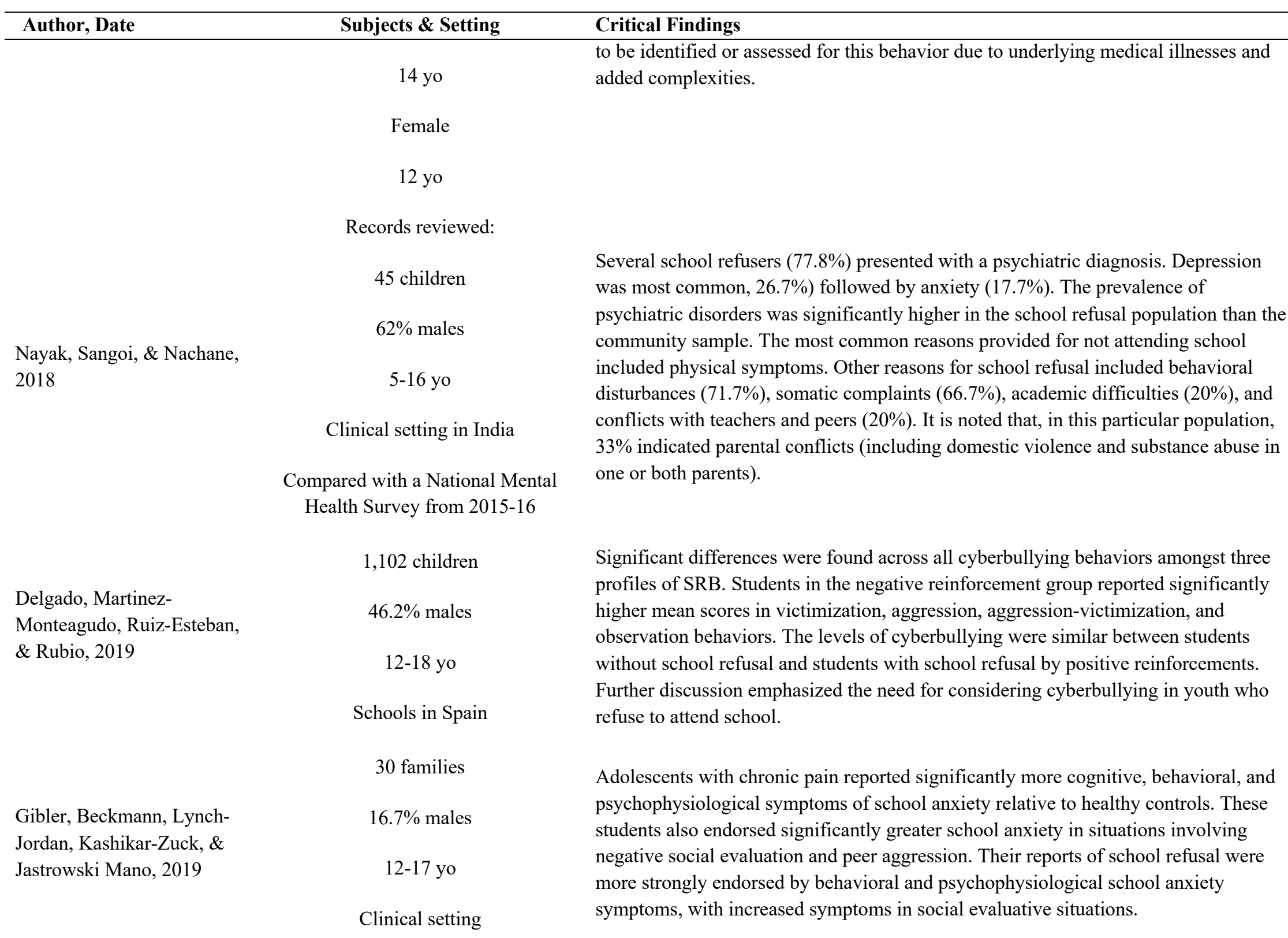


Compared to an age- and sexmatched control group

1,315 children

Gonzálvez, Díaz-Herrero, Vicent, Sanmartín, Pérez-

Sánchez, \& García-

Fernández, 2019

Gonzálvez, Inglés, Kearney, Sanmartín, Vicent \&

García-Fernández, 2019

\section{$57.6 \%$ males}

12-18 yo

11 high schools in Spain

1,842 children

$47 \%$ males

15-18 yo

Secondary school in Spain

\section{1,212 children}

8-11 yo

17 schools in Spain
Moderately high, moderately low, mixed and non-school refusal profiles were compared with each other based on scores from eleven dimensions of a self-concept. Results indicated that the mixed school refusal behavior group was the most maladaptive profile with the lowest mean scores on self-concept while non-school refusal and moderately low school refusal behavior groups revealed higher scores in all dimensions of self-concept.

Separate school refusal profiles were used to examine the relationships between three dimensions of social anxiety. Across 4 profiles of school refusal, the high school refusal behavior group showed higher scores in all subscales of social anxiety, and the lowest scores on perception of good family functioning. Results indicate students who refuse to attend school are at a higher risk of developing social anxiety problems and manifesting family conflicts.

Across 4 school refusal profiles, students in the non-school refusal group and school refusers by tangible reinforcements group presented better social functioning when compared with the rest of the groups. Students with mixed and negative reinforcements maintaining school refusal behavior may benefit from targeted interventions for acquiring social and communication skills. 


\section{Appendix E}

\section{Proserc covvum \\ UNIVERSITY OF CALIFORNIA \\ SANTA BARBARA}

\section{$\underline{\text { Data Request Form }}$}

Date of request: $08 / 18 / 2020$

Name: Zack Maupin

Email: Zmaupin@chapman.edu

Affiliation and Department: Chapman University (doctoral student in school of education)

- Please describe your connection to the Primary Investigators (PIs) of the grant: Dr. Amy-Jane Griffiths at Chapman University, my advisor, is a past colleague and acquaintance to Dr. Furlong.

- Which dataset(s) are you requesting? Which variables and instruments do you intend to use?

Please include all covariates/demographic variables and outcome variables.

- Data for high school secondary students:

$\checkmark$ 2017-18 and 2018-19 CHKS-SCM demographic questions: 3-7, 9, 10, 12, 13, 207

$\checkmark$ 2017-18 and 2018-19 CHKS-SCM items for behavior indicators (grades, cigarette, vaping, drinking, marijuana, suicidal ideation, sadness), questions 20, 70, 72, 74, 75, 131,130 (respectively)

$\checkmark$ 2017-18 and 2018-19 CHKS-SCM attendance questions: 21, 22, 23

$\checkmark$ Concurrent Social-Emotional Health Survey-Secondary module

$\checkmark$ Concurrent Social Emotional Distress Scale-Secondary

- How will data being requested be used?

The data requested is intended to support my dissertation study. The study intends to promote a dual-factor model approach for conceptualizing school attendance problems. Results will contribute towards more effective assessments that consider mental health and subjective wellbeing (complete mental health) in students who struggle with attendance.

- We encourage students to present and publish their work! Please check the following boxes to indicate that you understand and agree to abide by the terms of the data sharing agreement. 
$\bigotimes 1$. I understand that this data is owned by Project CoVitality and will be used for research purposes only. I agree to not use the data for purposes other than which I have stated here. If my intentions change, I will submit an amended data sharing agreement.

$\bigotimes 2$. I understand that I will receive access to the data via a personal link to Box, and I will not download the data onto my computer. I will save all my datafiles in Box and will no longer be able to access the data once my contract expires.

$\bigotimes 3$. I understand that I am using these data with permission from the PIs of the grant. I understand that if I am interested in publishing or presenting my working outside of a course assignment, I will first submit a formal proposal outside of class to the PIs on the grant detailing my research question(s) and proposed analyses.

$\bigotimes 4$. By using these data, I understand that if I intend to publish or present my work outside of an assignment for a course, a PI on the grant should be offered authorship.

$\bigotimes 5$. If my paper is accepted for publication or a presentation, I agree to include the following statement: "The research reported here was supported in part by the Institute of Education Sciences, U.S. Department of Education, through Grant \#R305A160157 to the University of California, Santa Barbara. The opinions expressed are those of the authors and do not represent views of the Institute of Education Sciences or the U.S. Department of Education."

Do you intend to submit your work for a presentation or publication outside of class?

Yes: $₫$ No: $\square$ Maybe:

If you intend to submit somewhere, when, and where (hypotheticals are fine): School

Psychology Quarterly, School Psychology Review, or Psychology in the Schools

Please sign the form by typing your name here: Zachary D. Maupin

To submit the form, please send it via e-mail to the PIs at the email addresses below. When you receive approval via email from one the PIs, you may begin your research.

Mike Furlong: mfurlong@ucsb.edu

Karen Nylund-Gibson: knylund@education.ucsb.edu

Erin Dowdy: edowdy@ucsb.edu 


\section{Appendix F}

Frequency Distribution for SEHS-S Scores from Question 19 Responses

\begin{tabular}{lccc}
\hline $\begin{array}{l}\text { Question 19: In the past 30 days, how often did } \\
\text { you miss an entire day of school for any reason? }\end{array}$ & $n$ & $\begin{array}{c}\text { Skewness } \\
\text { (Std. Error) }\end{array}$ & $\begin{array}{c}\text { Kurtosis } \\
\text { (Std. Error) }\end{array}$ \\
\hline O days & 45509 & -.441 & .033 \\
& & $(.011)$ & $(.023)$ \\
I day & 21669 & -.413 & .029 \\
& & $(.017)$ & $(.033)$ \\
2 days & 14155 & -.332 & .001 \\
& & $(.021)$ & $(.041)$ \\
3 or more days & 12428 & -.333 & -.050 \\
& & $(.022)$ & $(.044)$ \\
\hline
\end{tabular}

Histogram Charts for Distribution of SEHS-S Scores for Question 19 Responses

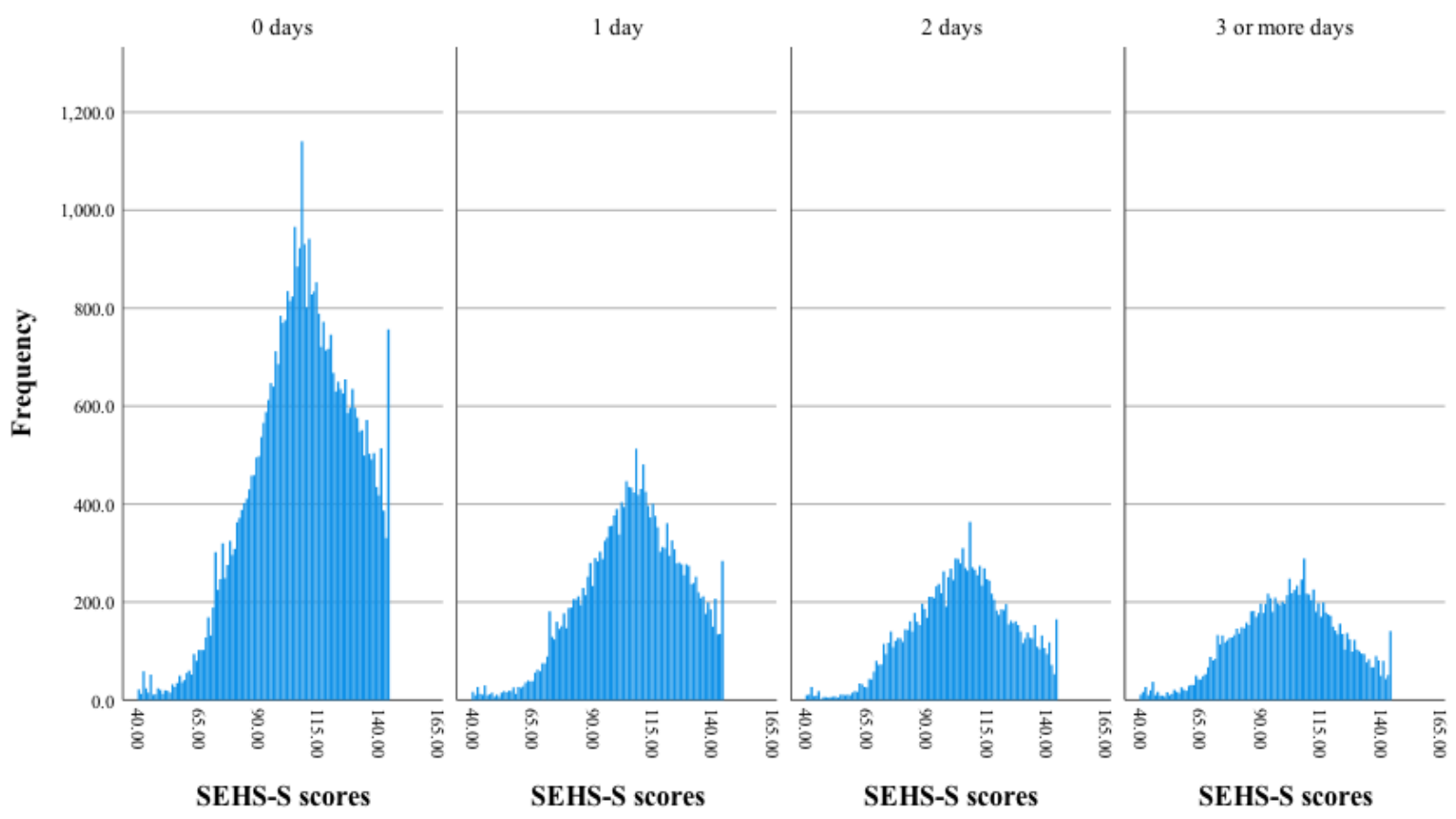




\section{Appendix G}

Frequency Distribution for SEHS-S Scores from Question 21 Responses

\begin{tabular}{|c|c|c|c|}
\hline $\begin{array}{l}\text { Question 21: During the past } 12 \text { months, about how } \\
\text { many times did you skip school or cut classes? }\end{array}$ & $n$ & $\begin{array}{l}\text { Skewness } \\
\text { (Std. Error) }\end{array}$ & $\begin{array}{l}\text { Kurtosis } \\
\text { (Std. Error) }\end{array}$ \\
\hline 0 times & 70494 & $\begin{array}{l}-.438 \\
(.009)\end{array}$ & $\begin{array}{l}.051 \\
(.018)\end{array}$ \\
\hline 1-2 times & 11403 & $\begin{array}{l}-.312 \\
(.023)\end{array}$ & $\begin{array}{l}-.057 \\
(.046)\end{array}$ \\
\hline A few times & 7756 & $\begin{array}{l}-.294 \\
(.028)\end{array}$ & $\begin{array}{l}-.028 \\
(.056)\end{array}$ \\
\hline Once a month & 840 & $\begin{array}{l}-.446 \\
(.084)\end{array}$ & $\begin{array}{l}.237 \\
(.169)\end{array}$ \\
\hline Twice a month & 1175 & $\begin{array}{l}-.298 \\
(.071)\end{array}$ & $\begin{array}{l}-.012 \\
(.143)\end{array}$ \\
\hline Once a week & 798 & $\begin{array}{l}-.178 \\
(.087)\end{array}$ & $\begin{array}{l}-.073 \\
(.173)\end{array}$ \\
\hline More than once a week & 1475 & $\begin{array}{l}-.126 \\
(.064)\end{array}$ & $\begin{array}{l}-.109 \\
(.127)\end{array}$ \\
\hline
\end{tabular}

Histogram Charts for Distribution of SEHS-S Scores from Question 21 Responses

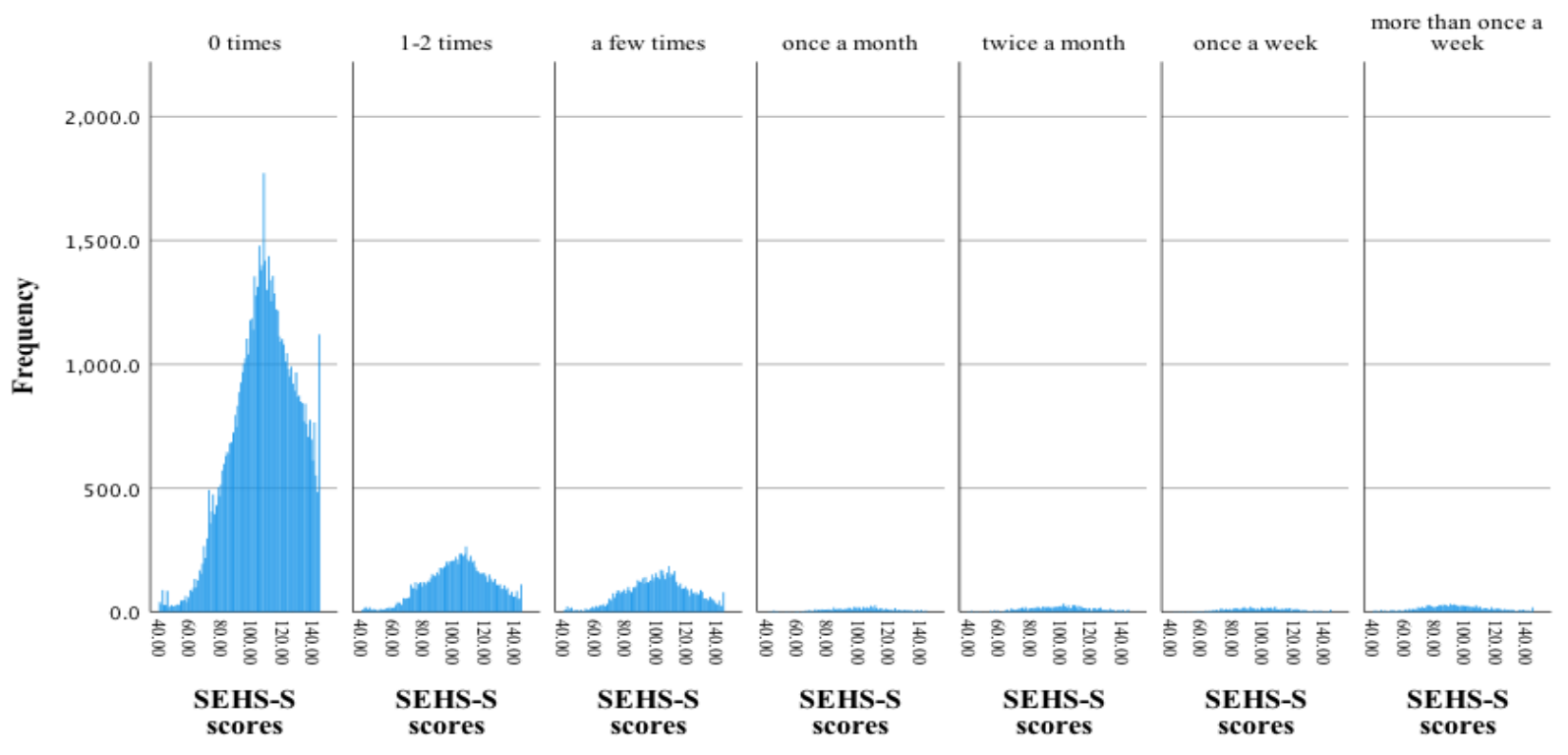




\section{Appendix H}

Frequency Distribution for SEDS-S Scores from Question 19 Responses

\begin{tabular}{lccc}
\hline $\begin{array}{l}\text { Question 19: In the past 30 days, how often did } \\
\text { you miss an entire day of school for any reason? }\end{array}$ & $n$ & $\begin{array}{c}\text { Skewness } \\
\text { (Std. Error) }\end{array}$ & $\begin{array}{c}\text { Kurtosis } \\
\text { (Std. Error) }\end{array}$ \\
\hline O days & 49486 & -.441 & .033 \\
& & $(.011)$ & $(.023)$ \\
1 day & 23563 & -.413 & .029 \\
& & $(.017)$ & $(.033)$ \\
2 days & 15602 & -.332 & .001 \\
& & $(.021)$ & $(.041)$ \\
3 or more days & 13808 & -.333 & -.050 \\
& & $(.022)$ & $(.044)$ \\
\hline
\end{tabular}

Histogram Charts for Distribution of SEDS-S Scores from Question 19 Responses

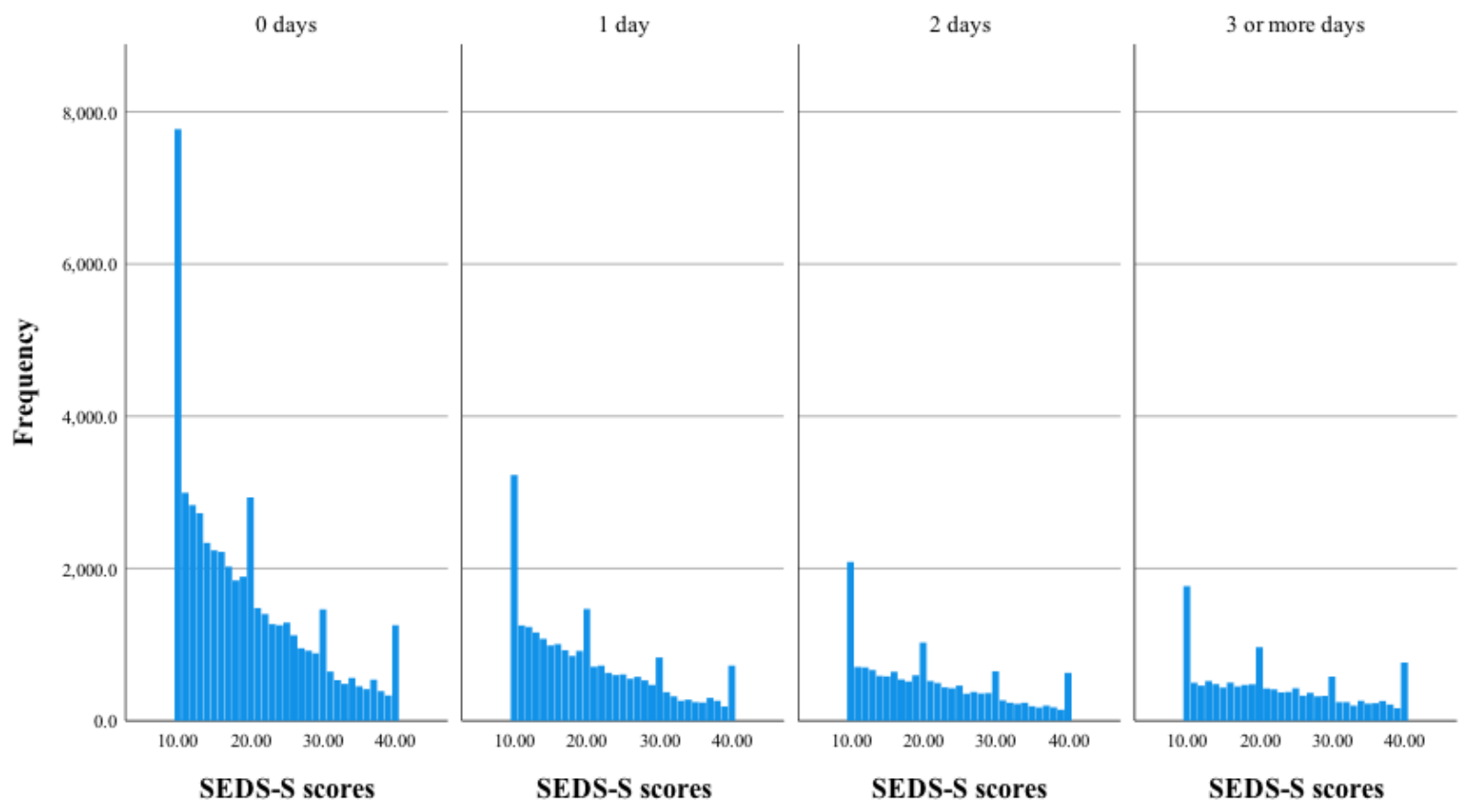




\section{Appendix I}

Frequency Distribution for SEDS-S Scores from Question 21 Responses

\begin{tabular}{lccc}
\hline $\begin{array}{l}\text { Question 21: During the past } 12 \text { months, about how } \\
\text { many times did you skip school or cut classes? }\end{array}$ & $n$ & $\begin{array}{c}\text { Skewness } \\
\text { (Std. Error) }\end{array}$ & $\begin{array}{c}\text { Kurtosis } \\
\text { (Std. Error) }\end{array}$ \\
\hline O times & 76969 & .779 & -.354 \\
& & $(.009)$ & $(.018)$ \\
$1-2$ times & 12470 & .551 & -.674 \\
& & $(.022)$ & $(.044)$ \\
A few times & 8504 & .459 & -.812 \\
& & $(.027)$ & $(.053)$ \\
Once a month & 944 & .342 & -1.006 \\
& & $(.080)$ & $(.159)$ \\
Twice a month & 1279 & .263 & -1.008 \\
& & $(.068)$ & $(.137)$ \\
Once a week & 860 & .262 & -1.058 \\
& & $(.083)$ & $(.167)$ \\
More than once a week & 1636 & .235 & -1.908 \\
\end{tabular}

Histogram Charts for Distribution of SEDS-S Scores from Question 21 Responses

Group 2: During the past 12 months, about how many times did you skip school or cut classes?

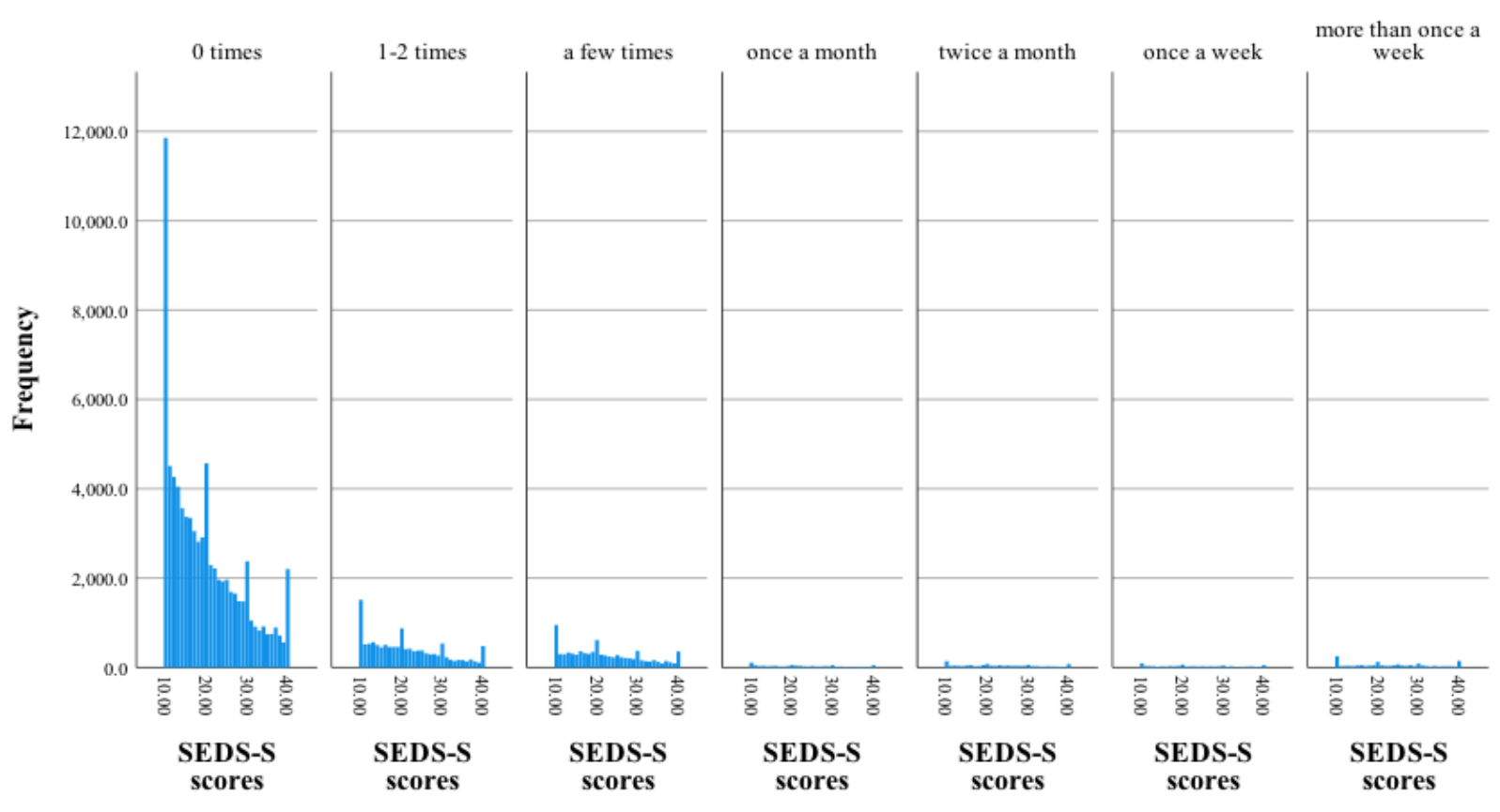




\section{Appendix J}

Cross-Tabs for Grades and Question 19 Responses

\begin{tabular}{lcccccc}
\hline \multirow{2}{*}{ Grade level } & 0 days & 1 day & 2 days & 3 or more days & Total \\
\hline \multirow{2}{*}{ Seventh } & $n$ & 14743 & 5891 & 3717 & 3248 & 27599 \\
& $(\%)$ & $(53.4 \%)$ & $(21.3 \%)$ & $(13.5 \%)$ & $(11.8 \%)$ & $(100.0 \%)$ \\
Eighth & $n$ & 2735 & 1349 & 880 & 711 & 5675 \\
& $(\%)$ & $(48.2 \%)$ & $(23.8 \%)$ & $(15.5 \%)$ & $(12.5 \%)$ & $(100.0 \%)$ \\
Ninth & $n$ & 14892 & 6693 & 4219 & 3705 & 29509 \\
& $(\%)$ & $(50.5 \%)$ & $(22.7 \%)$ & $(14.3 \%)$ & $(12.6 \%)$ & $(100.0 \%)$ \\
Tenth & $n$ & 5019 & 2381 & 1645 & 1450 & 10495 \\
& $(\%)$ & $(47.8 \%)$ & $(22.7 \%)$ & $(15.7 \%)$ & $(13.8 \%)$ & $(100.0 \%)$ \\
Eleventh & $n$ & 10782 & 6180 & 4272 & 3809 & 25043 \\
& $(\%)$ & $(43.1 \%$ & $(24.7 \%)$ & $(17.1 \%)$ & $(15.2 \%)$ & $(100.0 \%)$ \\
Twelfth & $n$ & 3249 & 2040 & 1546 & 1495 & 8330 \\
& $(\%)$ & $(39.0 \%)$ & $(24.5 \%)$ & $(18.6 \%)$ & $(17.9 \%)$ & $(100.0 \%)$ \\
Total & $n$ & 51420 & 24534 & 16279 & 14418 & 106651 \\
& $(\%)$ & $(48.2 \%)$ & $(23.0 \%)$ & $(15.3 \%)$ & $(13.5 \%)$ & $(100.0 \%)$ \\
\hline
\end{tabular}

Cross-Tabs for Grades and Question 21 Responses

\begin{tabular}{lccccccccc}
\hline \multirow{2}{*}{ Grade level } & O times & $\begin{array}{c}\text { 1-2 } \\
\text { times }\end{array}$ & $\begin{array}{c}\text { Afew } \\
\text { times }\end{array}$ & $\begin{array}{c}\text { Once } a \\
\text { month }\end{array}$ & $\begin{array}{c}\text { Twice a } \\
\text { month }\end{array}$ & $\begin{array}{c}\text { Once a } \\
\text { week }\end{array}$ & $\begin{array}{c}\text { More than } \\
\text { once a week }\end{array}$ & Total \\
\hline \multirow{2}{*}{ Seventh } & $n$ & 22205 & 2996 & 1876 & 165 & 179 & 74 & 203 & 27698 \\
& $(\%)$ & $(80.2 \%)$ & $(10.8 \%)$ & $(6.8 \%)$ & $(0.6 \%)$ & $(0.6 \%)$ & $(0.3 \%)$ & $(0.7 \%)$ & $(100.0 \%)$ \\
Eighth & $n$ & 4627 & 577 & 335 & 44 & 37 & 25 & 48 & 5693 \\
& $(\%)$ & $(81.3 \%)$ & $(10.1 \%)$ & $(5.9 \%)$ & $(0.8 \%)$ & $(0.6 \%)$ & $(0.4 \%)$ & $(0.8 \%)$ & $(100.0 \%)$ \\
\multirow{2}{*}{ Ninth } & $n$ & 23131 & 3241 & 2099 & 201 & 251 & 162 & 458 & 29543 \\
& $(\%)$ & $(78.3 \%)$ & $(11.0 \%)$ & $(7.1 \%)$ & $(0.7 \%)$ & $(0.8 \%)$ & $(0.5 \%)$ & $(1.6 \%)$ & $(100.0 \%)$ \\
Tenth & $n$ & 7882 & 1313 & 823 & 86 & 105 & 90 & 209 & 10508 \\
& $(\%)$ & $(75.0 \%)$ & $(12.5 \%)$ & $(7.8 \%)$ & $(0.8 \%)$ & $(1.0 \%)$ & $(0.9 \%)$ & $(2.0 \%)$ & $(100.0 \%)$ \\
Eleventh & $n$ & 17089 & 3542 & 2663 & 340 & 509 & 353 & 578 & 25074 \\
& $(\%)$ & $(68.2 \%)$ & $(14.1 \%)$ & $(10.6 \%)$ & $(1.4 \%)$ & $(2.0 \%)$ & $(1.4 \%)$ & $(2.3 \%)$ & $(100.0 \%)$ \\
\multirow{2}{*}{ Twelfth } & $n$ & 5083 & 1348 & 1074 & 141 & 254 & 204 & 237 & 8341 \\
& $(\%)$ & $(60.9 \%)$ & $(16.2 \%)$ & $(12.9 \%)$ & $(1.7 \%)$ & $(3.0 \%)$ & $(2.4 \%)$ & $(2.8 \%)$ & $(100.0 \%)$ \\
\multirow{2}{*}{ Total } & $n$ & 80017 & 13017 & 8870 & 977 & 1335 & 908 & 1733 & 106857 \\
& $(\%)$ & $(74.9 \%)$ & $(12.2 \%)$ & $(8.3 \%)$ & $(0.9 \%)$ & $(1.2 \%)$ & $(0.8 \%)$ & $(1.6 \%)$ & $(100.0 \%)$ \\
\hline
\end{tabular}




\section{Appendix K}

Cross-Tabs for Grades and Question 19 Responses

\begin{tabular}{lcccccc}
\hline Gender & & 0 days & 1 day & 2 days & 3 or more days & Total \\
\hline \multirow{2}{*}{ Male } & $n$ & 26527 & 11444 & 7085 & 6002 & 51058 \\
& $(\%)$ & $(52.0 \%)$ & $(22.4 \%)$ & $(13.9 \%)$ & $(11.8 \%)$ & $(100.0 \%)$ \\
\multirow{2}{*}{ Female } & $n$ & 24219 & 12687 & 8939 & 8171 & 54016 \\
& $(\%)$ & $(44.8 \%)$ & $(23.5 \%)$ & $(16.5 \%)$ & $(15.1 \%)$ & $(100.0 \%)$ \\
\multirow{2}{*}{ Total } & $n$ & 50746 & 24131 & 16024 & 14173 & 105074 \\
& $(\%)$ & $(48.3 \%)$ & $(23.0 \%)$ & $(15.3 \%)$ & $(13.5 \%)$ & $(100.0 \%)$ \\
\hline
\end{tabular}

Cross-Tabs for Gender and Question 21 Responses

\begin{tabular}{lccccccccc}
\hline Gender & & O times & 1-2 times & $\begin{array}{c}\text { Afew } \\
\text { times }\end{array}$ & $\begin{array}{c}\text { Once a } \\
\text { month }\end{array}$ & $\begin{array}{c}\text { Twice a } \\
\text { month }\end{array}$ & $\begin{array}{c}\text { Once a } \\
\text { week }\end{array}$ & $\begin{array}{c}\text { More than } \\
\text { once a week }\end{array}$ & Total \\
\hline \multirow{2}{*}{ Male } & $n$ & 38777 & 6137 & 4024 & 483 & 576 & 390 & 789 & 51176 \\
& $(\%)$ & $(75.8 \%)$ & $(12.0 \%)$ & $(7.9 \%)$ & $(0.9 \%)$ & $(1.1 \%)$ & $(0.8 \%)$ & $(1.5 \%)$ & $(100.0 \%)$ \\
\multirow{2}{*}{ Female } & $n$ & 40125 & 6663 & 4700 & 473 & 729 & 500 & 908 & 54098 \\
& $(\%)$ & $(74.2 \%)$ & $(12.3 \%)$ & $(8.7 \%)$ & $(0.9 \%)$ & $(1.3 \%)$ & $(0.9 \%)$ & $(1.7 \%)$ & $(100.0 \%)$ \\
\multirow{2}{*}{ Total } & $n$ & 78902 & 12800 & 8724 & 956 & 1305 & 890 & 1697 & 105274 \\
& $(\%)$ & $(74.9 \%)$ & $(12.2 \%)$ & $(8.3 \%)$ & $(0.9 \%)$ & $(1.2 \%)$ & $(0.8 \%)$ & $(1.6 \%)$ & $(100.0 \%)$ \\
\hline
\end{tabular}

Cross-Tabs for Transgender and Question 19 Responses

\begin{tabular}{lcccccc}
\hline Transgender & & 0 days & 1 day & 2 days & 3 or more days & Total \\
\hline \multirow{2}{*}{ No, I am not transgender } & $n$ & 47569 & 22566 & 14779 & 12943 & 97857 \\
& $(\%)$ & $(48.6 \%)$ & $(23.1 \%)$ & $(15.1 \%)$ & $(13.2 \%)$ & $(100.0 \%)$ \\
Yes, I am transgender & $n$ & 273 & 172 & 149 & 161 & 755 \\
& $(\%)$ & $(36.2 \%)$ & $(22.8 \%)$ & $(19.7 \%)$ & $(21.3 \%)$ & $(100.0 \%)$ \\
I am not sure if I am transgender & $n$ & 747 & 311 & 245 & 241 & 1544 \\
& $(\%)$ & $(48.4 \%)$ & $(20.1 \%)$ & $(15.9 \%)$ & $(15.6 \%)$ & $(100.0 \%)$ \\
Declined to respond & $n$ & 1254 & 532 & 345 & 386 & 2517 \\
& $(\%)$ & $(49.8 \%)$ & $(21.1 \%)$ & $(13.7 \%)$ & $(15.3 \%)$ & $(100.0 \%)$ \\
Total & $n$ & 49843 & 23581 & 15518 & 13731 & 102673 \\
& $(\%)$ & $(48.5 \%)$ & $(23.0 \%)$ & $(15.1 \%)$ & $(13.4 \%)$ & $(100.0 \%)$ \\
\hline
\end{tabular}


Cross-Tabs for Transgender and Question 21 Responses

\begin{tabular}{|c|c|c|c|c|c|c|c|c|c|}
\hline Transgender & & 0 times & $\begin{array}{c}1-2 \\
\text { times }\end{array}$ & $\begin{array}{l}\text { Afew } \\
\text { times }\end{array}$ & $\begin{array}{l}\text { Once a } \\
\text { month }\end{array}$ & $\begin{array}{c}\text { Twice a } \\
\text { month }\end{array}$ & $\begin{array}{c}\text { Once a } \\
\text { week }\end{array}$ & $\begin{array}{c}\text { More than } \\
\text { once a } \\
\text { week }\end{array}$ & Total \\
\hline $\begin{array}{l}\text { No, I am not } \\
\text { transgender }\end{array}$ & $\begin{array}{c}n \\
(\%)\end{array}$ & $\begin{array}{c}73562 \\
(75.0 \%)\end{array}$ & $\begin{array}{c}11911 \\
(12.2 \%)\end{array}$ & $\begin{array}{c}8098 \\
(8.3 \%)\end{array}$ & $\begin{array}{c}871 \\
(0.9 \%)\end{array}$ & $\begin{array}{c}1217 \\
(1.2 \%)\end{array}$ & $\begin{array}{c}828 \\
(0.8 \%)\end{array}$ & $\begin{array}{c}1541 \\
(1.6 \%)\end{array}$ & $\begin{array}{c}98028 \\
(100.0 \%)\end{array}$ \\
\hline $\begin{array}{l}\text { Yes, I am } \\
\text { transgender }\end{array}$ & $\begin{array}{c}n \\
(\%)\end{array}$ & $\begin{array}{c}474 \\
(62.9 \%)\end{array}$ & $\begin{array}{c}116 \\
(15.4 \%)\end{array}$ & $\begin{array}{c}77 \\
(10.2 \%)\end{array}$ & $\begin{array}{c}20 \\
(2.7 \%)\end{array}$ & $\begin{array}{c}23 \\
(3.1 \%)\end{array}$ & $\begin{array}{c}11 \\
(1.5 \%)\end{array}$ & $\begin{array}{c}33 \\
(4.4 \%)\end{array}$ & $\begin{array}{c}754 \\
(100.0 \%)\end{array}$ \\
\hline $\begin{array}{l}\text { I am not sure if I } \\
\text { am transgender }\end{array}$ & $\begin{array}{c}n \\
(\%)\end{array}$ & $\begin{array}{c}1083 \\
(69.9 \%)\end{array}$ & $\begin{array}{c}204 \\
(13.2 \%)\end{array}$ & $\begin{array}{c}152 \\
(9.8 \%)\end{array}$ & $\begin{array}{c}26 \\
(1.7 \%)\end{array}$ & $\begin{array}{c}23 \\
(1.5 \%)\end{array}$ & $\begin{array}{c}18 \\
(1.2 \%)\end{array}$ & $\begin{array}{c}44 \\
(2.8 \%)\end{array}$ & $\begin{array}{c}1550 \\
(100.0 \%)\end{array}$ \\
\hline $\begin{array}{l}\text { Declined to } \\
\text { respond }\end{array}$ & $\begin{array}{c}n \\
(\%)\end{array}$ & $\begin{array}{c}1859 \\
(73.7 \%)\end{array}$ & $\begin{array}{c}303 \\
(12.0 \%)\end{array}$ & $\begin{array}{c}232 \\
(9.2 \%)\end{array}$ & $\begin{array}{c}24 \\
(1.0 \%)\end{array}$ & $\begin{array}{c}26 \\
(1.0 \%)\end{array}$ & $\begin{array}{c}25 \\
(1.0 \%)\end{array}$ & $\begin{array}{c}52 \\
(2.1 \%)\end{array}$ & $\begin{array}{c}2521 \\
(100.0 \%)\end{array}$ \\
\hline Total & $\begin{array}{c}n \\
(\%)\end{array}$ & $\begin{array}{c}76978 \\
(74.8 \%)\end{array}$ & $\begin{array}{l}12534 \\
(12.2)\end{array}$ & $\begin{array}{c}8559 \\
(8.3 \%)\end{array}$ & $\begin{array}{c}941 \\
(0.9 \%)\end{array}$ & $\begin{array}{c}1289 \\
(1.3 \%)\end{array}$ & $\begin{array}{c}882 \\
(0.9 \%)\end{array}$ & $\begin{array}{c}1670 \\
(1.6 \%)\end{array}$ & $\begin{array}{c}102853 \\
(100.0 \%)\end{array}$ \\
\hline
\end{tabular}

Cross-Tabs for Sexual Orientation and Question 19 Responses

\begin{tabular}{lcccccc}
\hline Sexual orientation & & 0 days & 1 day & 2 days & $\begin{array}{c}3 \text { or more } \\
\text { days }\end{array}$ & Total \\
& & & & & 10983 & 85178 \\
Straight (not gay) & $n$ & 41723 & 19717 & 12755 & $17 \%)$ & $(100.0 \%)$ \\
& $(\%)$ & $(49.0 \%)$ & $(23.1 \%)$ & $(15.0 \%)$ & $(12.9 \%)$ & 1546 \\
Gay or lesbian & $n$ & 639 & 329 & 293 & 285 & $(100.0 \%)$ \\
& $(\%)$ & $(41.3 \%)$ & $(21.3 \%)$ & $(19.0 \%)$ & $(18.4 \%)$ & 5647 \\
Bisexual & $n$ & 2287 & 1309 & 1011 & 1040 & $(100.0 \%)$ \\
& $(\%)$ & $(40.5 \%)$ & $(23.2 \%)$ & $(17.9 \%)$ & $(18.4 \%)$ & 4803 \\
I am not sure yet & $n$ & 2405 & 1056 & 665 & 677 & $(100.0 \%)$ \\
Something else & $(\%)$ & $(50.1 \%)$ & $(22.0 \%)$ & $(13.8 \%)$ & $(14.1 \%)$ & 1486 \\
& $n$ & 680 & 332 & 244 & 230 & $(100.0 \%)$ \\
Declined to respond & $(\%)$ & $(45.8 \%)$ & $(22.3 \%)$ & $(16.4 \%)$ & $(15.5 \%)$ & 3999 \\
& $n$ & 2096 & 841 & 547 & 515 & $(100.0 \%)$ \\
Total & $(\%)$ & $(52.4 \%)$ & $(21.0 \%)$ & $(13.7 \%)$ & $(12.9 \%)$ & 102659 \\
& $n$ & 49830 & 23584 & 15515 & 13730 & $(100.0 \%)$ \\
\hline
\end{tabular}


Cross-Tabs for Sexual Orientation and Question 21 Responses

\begin{tabular}{lccccccccc}
\hline Sexual orientation & O times & 1-2 times & $\begin{array}{c}\text { Afew } \\
\text { times }\end{array}$ & $\begin{array}{c}\text { Once a } \\
\text { month }\end{array}$ & $\begin{array}{c}\text { Twice a } \\
\text { month }\end{array}$ & $\begin{array}{c}\text { Once a } \\
\text { week }\end{array}$ & $\begin{array}{c}\text { More than } \\
\text { once a week }\end{array}$ & Total \\
\hline Straight & $n$ & 64415 & 10228 & 6933 & 740 & 1028 & 713 & 1282 & 85339 \\
(not gay) & $(\%)$ & $(75.5 \%)$ & $(12.0 \%)$ & $(8.1 \%)$ & $(0.9 \%)$ & $(1.2 \%)$ & $(0.8 \%)$ & $(1.5 \%)$ & $(100.0 \%)$ \\
Gay or & $n$ & 1035 & 221 & 162 & 24 & 26 & 20 & 55 & 1543 \\
lesbian & $(\%)$ & $(67.1 \%)$ & $(14.3 \%)$ & $(10.5 \%)$ & $(1.6 \%)$ & $(1.7 \%)$ & $(1.3 \%)$ & $(3.6 \%)$ & $(100.0 \%)$ \\
Bisexual & $n$ & 3734 & 842 & 627 & 76 & 124 & 69 & 175 & 5647 \\
I am not & $n$ & 3614 & 579 & 397 & 49 & 56 & 40 & 74 & 4809 \\
sure yet & $(\%)$ & $(75.2 \%)$ & $(12.0 \%)$ & $(8.3 \%)$ & $(1.0 \%)$ & $(1.2 \%)$ & $(0.8 \%)$ & $(1.5 \%)$ & $(100.0 \%)$ \\
Something & $n$ & 1062 & 195 & 136 & 19 & 25 & 18 & 36 & 1491 \\
else & $(\%)$ & $(71.2 \%)$ & $(13.1 \%)$ & $(9.1 \%)$ & $(1.3 \%)$ & $(1.7 \%)$ & $(1.2 \%)$ & $(2.4 \%)$ & $(100.0 \%)$ \\
Declined to & $n$ & 3114 & 460 & 300 & 31 & 34 & 22 & 50 & 4011 \\
respond & $(\%)$ & $(77.6 \%)$ & $(11.5 \%)$ & $(7.5 \%)$ & $(0.8 \%)$ & $(0.8 \%)$ & $(0.5 \%)$ & $(1.2 \%)$ & $(100.0 \%)$ \\
Total & $n$ & 76974 & 12525 & 8555 & 939 & 1293 & 882 & 1672 & 102840 \\
& $(\%)$ & $(74.8 \%)$ & $(12.2 \%)$ & $(8.3 \%)$ & $(0.9 \%)$ & $(1.3 \%)$ & $(0.9 \%)$ & $(1.6 \%)$ & $(100.0 \%)$ \\
\hline
\end{tabular}




\section{Appendix L}

Cross-Tabs for Hispanic/Latinx and Question 19 Responses

\begin{tabular}{lcccccc}
\hline Hispanic/Latinx & & O days & 1 day & 2 days & 3 or more days & Total \\
\cline { 1 - 1 } & $n$ & 23187 & 11683 & 8440 & 7636 & 50946 \\
Yes & $(\%)$ & $(45.5 \%)$ & $(22.9 \%)$ & $(16.6 \%)$ & $(15.0 \%)$ & $(100.0 \%)$ \\
& $n$ & 27853 & 12684 & 7733 & 6686 & 54956 \\
No & $(\%)$ & $(50.7 \%)$ & $(23.1 \%)$ & $(14.1 \%)$ & $(12.2 \%)$ & $(100.0 \%)$ \\
& $n$ & 51040 & 24367 & 16173 & 14322 & 105902 \\
\multirow{2}{*}{ Total } & $(\%)$ & $(48.2 \%)$ & $(23.0 \%)$ & $(15.3 \%)$ & $(13.5 \%)$ & $(100.0 \%)$ \\
& & & & & &
\end{tabular}

Cross-Tabs for Hispanic/Latinx and Question 21 Responses

\begin{tabular}{lccccccccc}
\hline Hispanic/Latinx & O times & 1-2 times & $\begin{array}{c}\text { Afew } \\
\text { times }\end{array}$ & $\begin{array}{c}\text { Once a } \\
\text { month }\end{array}$ & $\begin{array}{c}\text { Twice a } \\
\text { month }\end{array}$ & $\begin{array}{c}\text { Once a } \\
\text { week }\end{array}$ & $\begin{array}{c}\text { More than } \\
\text { once a week }\end{array}$ & Total \\
\hline \multirow{2}{*}{ Yes } & $n$ & 37222 & 6380 & 4691 & 463 & 645 & 520 & 1145 & 51066 \\
& $(\%)$ & $(72.9 \%)$ & $(12.5 \%)$ & $(9.2 \%)$ & $(0.9 \%)$ & $(1.3 \%)$ & $(1.0 \%)$ & $(2.2 \%)$ & $(100.0 \%)$ \\
\multirow{2}{*}{ No } & $n$ & 42217 & 6550 & 4112 & 509 & 684 & 382 & 583 & 55037 \\
& $(\%)$ & $(76.7 \%)$ & $(11.9 \%)$ & $(7.5 \%)$ & $(0.9 \%)$ & $(1.2 \%)$ & $(0.7 \%)$ & $(1.1 \%)$ & $(100.0 \%)$ \\
\multirow{2}{*}{ Total } & $n$ & 79439 & 12930 & 8803 & 972 & 1329 & 902 & 1728 & 106103 \\
& $(\%)$ & $(74.9 \%)$ & $(12.2 \%)$ & $(8.3 \%)$ & $(0.9 \%)$ & $(1.3 \%)$ & $(0.9 \%)$ & $(1.6 \%)$ & $(100.0 \%)$ \\
\hline
\end{tabular}

Cross-Tabs for Race/Ethnicity and Question 19 Responses

\begin{tabular}{|c|c|c|c|c|c|c|}
\hline Race/ethnicity & & 0 days & 1 day & 2 days & $\begin{array}{c}3 \text { or more } \\
\text { days }\end{array}$ & Total \\
\hline American Indian or & $n$ & 1399 & 665 & 496 & 496 & 3056 \\
\hline Alaska native & $(\%)$ & $(45.8 \%)$ & $(21.8 \%)$ & $(16.2 \%)$ & $(16.2 \%)$ & $(100.0 \%)$ \\
\hline \multirow{2}{*}{ Asian } & $n$ & 9556 & 2680 & 1358 & 977 & 14571 \\
\hline & $(\%)$ & $(65.6 \%)$ & $(18.4 \%)$ & $(9.3 \%)$ & $(6.7 \%)$ & $(100.0 \%)$ \\
\hline Black or & $n$ & 1372 & 605 & 494 & 477 & 2948 \\
\hline African American & $(\%)$ & $(46.5 \%)$ & $(20.5 \%)$ & $(16.8 \%)$ & $(16.2 \%)$ & $(100.0 \%)$ \\
\hline Native Hawaiian or & $n$ & 717 & 300 & 229 & 223 & 1469 \\
\hline Pacific Islander & $(\%)$ & $(48.8 \%)$ & $(20.4 \%)$ & $(15.6 \%)$ & $(15.2 \%)$ & $(100.0 \%)$ \\
\hline \multirow{2}{*}{ White } & $n$ & 15134 & 8606 & 5499 & 4715 & 33954 \\
\hline & $(\%)$ & $(44.6 \%)$ & $(25.3 \%)$ & $(16.2 \%)$ & $(13.9 \%)$ & $(100.0 \%)$ \\
\hline
\end{tabular}




\begin{tabular}{|c|c|c|c|c|c|c|}
\hline Race/ethnicity & & 0 days & 1 day & 2 days & $\begin{array}{c}3 \text { or more } \\
\text { days }\end{array}$ & Total \\
\hline $\begin{array}{l}\text { Mixed (two or more) } \\
\text { races }\end{array}$ & $n(\%)$ & $\begin{array}{c}19165 \\
(45.3 \%)\end{array}$ & $\begin{array}{c}9738 \\
(23.0 \%)\end{array}$ & $\begin{array}{c}6926 \\
(16.4 \%)\end{array}$ & $\begin{array}{c}6437 \\
(15.2 \%)\end{array}$ & $\begin{array}{c}42266 \\
(100.0 \%)\end{array}$ \\
\hline Total & $n(\%)$ & $\begin{array}{c}47343 \\
(48.2 \%)\end{array}$ & $\begin{array}{c}22594 \\
(23.0 \%)\end{array}$ & $\begin{array}{c}15002 \\
(15.3 \%)\end{array}$ & $\begin{array}{c}13325 \\
(13.6 \%)\end{array}$ & $\begin{array}{c}98264 \\
(100.0 \%)\end{array}$ \\
\hline
\end{tabular}

Cross-Tabs for Race/Ethnicity and Question 21 Responses

\begin{tabular}{|c|c|c|c|c|c|c|c|c|c|}
\hline Race/ethnicity & & 0 times & $\begin{array}{c}1-2 \\
\text { times }\end{array}$ & $\begin{array}{l}\text { Afew } \\
\text { times }\end{array}$ & $\begin{array}{l}\text { Once a } \\
\text { month }\end{array}$ & $\begin{array}{c}\text { Twice a } \\
\text { month }\end{array}$ & $\begin{array}{c}\text { Once a } \\
\text { week }\end{array}$ & $\begin{array}{c}\text { More than } \\
\text { once a } \\
\text { week }\end{array}$ & Total \\
\hline $\begin{array}{l}\text { American Indian } \\
\text { or Alaska native }\end{array}$ & $\begin{array}{c}n \\
(\%)\end{array}$ & $\begin{array}{c}2195 \\
(71.6 \%)\end{array}$ & $\begin{array}{c}400 \\
(13.1 \%)\end{array}$ & $\begin{array}{c}290 \\
(9.5 \%)\end{array}$ & $\begin{array}{c}35 \\
(1.1 \%)\end{array}$ & $\begin{array}{c}43 \\
(1.4 \%)\end{array}$ & $\begin{array}{c}31 \\
(1.0 \%)\end{array}$ & $\begin{array}{c}70 \\
(2.3 \%)\end{array}$ & $\begin{array}{c}3064 \\
(100.0 \%)\end{array}$ \\
\hline Asian & $\begin{array}{c}n \\
(\%)\end{array}$ & $\begin{array}{c}11468 \\
(78.7 \%)\end{array}$ & $\begin{array}{c}1796 \\
(12.3 \%)\end{array}$ & $\begin{array}{c}951 \\
(6.5 \%)\end{array}$ & $\begin{array}{c}95 \\
(0.7 \%)\end{array}$ & $\begin{array}{c}122 \\
(0.8 \%)\end{array}$ & $\begin{array}{c}43 \\
(0.3 \%)\end{array}$ & $\begin{array}{c}97 \\
(0.7 \%)\end{array}$ & $\begin{array}{c}14572 \\
(100.0 \%)\end{array}$ \\
\hline $\begin{array}{l}\text { Black or African } \\
\text { American }\end{array}$ & $\begin{array}{c}n \\
(\%)\end{array}$ & $\begin{array}{c}2211 \\
(74.7 \%)\end{array}$ & $\begin{array}{c}333 \\
(11.3 \%)\end{array}$ & $\begin{array}{c}269 \\
(9.1 \%)\end{array}$ & $\begin{array}{c}24 \\
(0.8 \%)\end{array}$ & $\begin{array}{c}32 \\
(1.1 \%)\end{array}$ & $\begin{array}{c}24 \\
(0.8 \%)\end{array}$ & $\begin{array}{c}67 \\
(2.3 \%)\end{array}$ & $\begin{array}{c}2960 \\
(100.0 \%)\end{array}$ \\
\hline $\begin{array}{l}\text { Native Hawaiian } \\
\text { or Pacific Islander }\end{array}$ & $\begin{array}{c}n \\
(\%)\end{array}$ & $\begin{array}{c}1061 \\
(72.1 \%)\end{array}$ & $\begin{array}{c}194 \\
(13.2 \%)\end{array}$ & $\begin{array}{c}123 \\
(8.4 \%)\end{array}$ & $\begin{array}{c}28 \\
(1.9 \%)\end{array}$ & $\begin{array}{c}21 \\
(1.4 \%)\end{array}$ & $\begin{array}{c}17 \\
(1.2 \%)\end{array}$ & $\begin{array}{c}28 \\
(1.9 \%)\end{array}$ & $\begin{array}{c}1472 \\
(100.0 \%)\end{array}$ \\
\hline White & $\begin{array}{c}n \\
(\%)\end{array}$ & $\begin{array}{c}25705 \\
(75.5 \%)\end{array}$ & $\begin{array}{c}3993 \\
(11.7 \%)\end{array}$ & $\begin{array}{c}2722 \\
(8.0 \%)\end{array}$ & $\begin{array}{c}359 \\
(1.1 \%)\end{array}$ & $\begin{array}{c}511 \\
(1.5 \%)\end{array}$ & $\begin{array}{c}307 \\
(0.9 \%)\end{array}$ & $\begin{array}{c}430 \\
(1.3 \%)\end{array}$ & $\begin{array}{c}34027 \\
(100.0 \%)\end{array}$ \\
\hline $\begin{array}{l}\text { Mixed (two or } \\
\text { more) races }\end{array}$ & $\begin{array}{c}n \\
(\%)\end{array}$ & $\begin{array}{c}31203 \\
(73.7 \%)\end{array}$ & $\begin{array}{c}5275 \\
(12.5 \%)\end{array}$ & $\begin{array}{c}3758 \\
(8.9 \%)\end{array}$ & $\begin{array}{c}361 \\
(0.9 \%)\end{array}$ & $\begin{array}{c}507 \\
(1.2 \%)\end{array}$ & $\begin{array}{c}397 \\
(0.9 \%)\end{array}$ & $\begin{array}{c}847 \\
(2.0 \%)\end{array}$ & $\begin{array}{c}42348 \\
(100.0 \%)\end{array}$ \\
\hline Total & $\begin{array}{c}n \\
(\%)\end{array}$ & $\begin{array}{c}73843 \\
(75.0 \%)\end{array}$ & $\begin{array}{c}11991 \\
(12.2 \%)\end{array}$ & $\begin{array}{c}8113 \\
(8.2 \%)\end{array}$ & $\begin{array}{c}902 \\
(0.9 \%)\end{array}$ & $\begin{array}{c}1236 \\
(1.3 \%)\end{array}$ & $\begin{array}{c}819 \\
(0.8 \%)\end{array}$ & $\begin{array}{c}1539 \\
(1.6 \%)\end{array}$ & $\begin{array}{c}98443 \\
(100.0 \%)\end{array}$ \\
\hline
\end{tabular}




\section{Appendix M}

Cross-Tabs for Parent Education and Question 19 Responses

\begin{tabular}{|c|c|c|c|c|c|c|}
\hline Parent education $^{\mathrm{a}}$ & & 0 days & 1 day & 2 days & $\begin{array}{c}3 \text { or more } \\
\text { days }\end{array}$ & Total \\
\hline Did not finish high school & $\begin{array}{c}n \\
(\%)\end{array}$ & $\begin{array}{c}5767 \\
(46.3 \%)\end{array}$ & $\begin{array}{c}2646 \\
(21.2 \%)\end{array}$ & $\begin{array}{c}1981 \\
(15.9 \%)\end{array}$ & $\begin{array}{c}2069 \\
(16.6 \%)\end{array}$ & $\begin{array}{c}12463 \\
(100.0 \%)\end{array}$ \\
\hline Graduated from high school & $\begin{array}{c}n \\
(\%)\end{array}$ & $\begin{array}{c}6280 \\
(42.7 \%)\end{array}$ & $\begin{array}{c}3471 \\
(23.6 \%)\end{array}$ & $\begin{array}{c}2586 \\
(17.6 \%)\end{array}$ & $\begin{array}{c}2370 \\
(16.1 \%)\end{array}$ & $\begin{array}{c}14707 \\
(100.0 \%)\end{array}$ \\
\hline $\begin{array}{l}\text { Attended college but did not complete a } \\
\text { four-year degree }\end{array}$ & $\begin{array}{c}n \\
(\%)\end{array}$ & $\begin{array}{c}5361 \\
(42.0 \%)\end{array}$ & $\begin{array}{c}3103 \\
(24.3 \%)\end{array}$ & $\begin{array}{c}2259 \\
(17.7 \%)\end{array}$ & $\begin{array}{c}2045 \\
(16.0 \%)\end{array}$ & $\begin{array}{c}12768 \\
(100.0 \%)\end{array}$ \\
\hline Graduated from college & $\begin{array}{c}n \\
(\%)\end{array}$ & $\begin{array}{c}25489 \\
(51.2 \%)\end{array}$ & $\begin{array}{c}11814 \\
(23.7 \%)\end{array}$ & $\begin{array}{c}6919 \\
(13.9 \%)\end{array}$ & $\begin{array}{c}5574 \\
(11.2 \%)\end{array}$ & $\begin{array}{c}49796 \\
(100.0 \%)\end{array}$ \\
\hline Total & $\begin{array}{c}n \\
(\%)\end{array}$ & $\begin{array}{c}42897 \\
(47.8 \%)\end{array}$ & $\begin{array}{c}21034 \\
(23.4 \%)\end{array}$ & $\begin{array}{c}13745 \\
(15.3 \%)\end{array}$ & $\begin{array}{c}12058 \\
(13.4 \%)\end{array}$ & $\begin{array}{c}89734 \\
(100.0 \%)\end{array}$ \\
\hline
\end{tabular}

a Students who reported don't know were excluded from analysis

Cross-Tabs for Parent Education and Question 21 Responses

\begin{tabular}{|c|c|c|c|c|c|c|c|c|c|}
\hline Parent education ${ }^{\mathrm{a}}$ & & 0 times & $\begin{array}{c}1-2 \\
\text { times }\end{array}$ & $\begin{array}{l}\text { A few } \\
\text { times }\end{array}$ & $\begin{array}{l}\text { Once a } \\
\text { month }\end{array}$ & $\begin{array}{c}\text { Twice } \\
a \\
\text { month }\end{array}$ & $\begin{array}{c}\text { Once a } \\
\text { week }\end{array}$ & $\begin{array}{c}\text { More } \\
\text { than } \\
\text { once a } \\
\text { week }\end{array}$ & Total \\
\hline $\begin{array}{l}\text { Did not finish } \\
\text { high school }\end{array}$ & $\begin{array}{c}n \\
(\%)\end{array}$ & $\begin{array}{c}8533 \\
(68.3 \%)\end{array}$ & $\begin{array}{c}1744 \\
(14.0 \%)\end{array}$ & $\begin{array}{c}1288 \\
(10.3 \%)\end{array}$ & $\begin{array}{c}137 \\
(1.1 \%)\end{array}$ & $\begin{array}{c}195 \\
(1.6 \%)\end{array}$ & $\begin{array}{c}168 \\
(1.3 \%)\end{array}$ & $\begin{array}{c}432 \\
(3.5 \%)\end{array}$ & $\begin{array}{c}12497 \\
(100.0 \%)\end{array}$ \\
\hline $\begin{array}{l}\text { Graduated from } \\
\text { high school }\end{array}$ & $\begin{array}{c}n \\
(\%)\end{array}$ & $\begin{array}{c}10603 \\
(72.0 \%)\end{array}$ & $\begin{array}{c}1922 \\
(13.0 \%)\end{array}$ & $\begin{array}{c}1344 \\
(9.1 \%)\end{array}$ & $\begin{array}{c}158 \\
(1.1 \%)\end{array}$ & $\begin{array}{c}220 \\
(1.5 \%)\end{array}$ & $\begin{array}{c}161 \\
(1.1 \%)\end{array}$ & $\begin{array}{c}327 \\
(2.2 \%)\end{array}$ & $\begin{array}{c}14735 \\
(100.0 \%)\end{array}$ \\
\hline $\begin{array}{l}\text { Attended college } \\
\text { but did not } \\
\text { complete a four- } \\
\text { year degree }\end{array}$ & $\begin{array}{c}n \\
(\%)\end{array}$ & $\begin{array}{c}9310 \\
(72.8 \%)\end{array}$ & $\begin{array}{c}1559 \\
(12.2 \%)\end{array}$ & $\begin{array}{c}1194 \\
(9.3 \%)\end{array}$ & $\begin{array}{c}128 \\
(1.0 \%)\end{array}$ & $\begin{array}{c}214 \\
(1.7 \%)\end{array}$ & $\begin{array}{c}145 \\
(1.1 \%)\end{array}$ & $\begin{array}{c}237 \\
(1.9 \%)\end{array}$ & $\begin{array}{c}12787 \\
(100.0 \%)\end{array}$ \\
\hline $\begin{array}{l}\text { Graduated from } \\
\text { college }\end{array}$ & $\begin{array}{c}n \\
(\%)\end{array}$ & $\begin{array}{c}38423 \\
(77.1 \%)\end{array}$ & $\begin{array}{c}5898 \\
(11.8 \%)\end{array}$ & $\begin{array}{c}3687 \\
(7.4 \%)\end{array}$ & $\begin{array}{c}464 \\
(0.9 \%)\end{array}$ & $\begin{array}{c}586 \\
(1.2 \%)\end{array}$ & $\begin{array}{c}334 \\
(0.7 \%)\end{array}$ & $\begin{array}{c}459 \\
(0.9 \%)\end{array}$ & $\begin{array}{c}49851 \\
(100.0 \%)\end{array}$ \\
\hline Total & $\begin{array}{c}n \\
(\%)\end{array}$ & $\begin{array}{c}66869 \\
(74.4 \%)\end{array}$ & $\begin{array}{c}11123 \\
(12.4 \%)\end{array}$ & $\begin{array}{c}7513 \\
(8.4 \%)\end{array}$ & $\begin{array}{c}887 \\
(1.0 \%)\end{array}$ & $\begin{array}{c}1215 \\
(1.4 \%)\end{array}$ & $\begin{array}{c}808 \\
(0.9 \%)\end{array}$ & $\begin{array}{c}1455 \\
(1.6 \%)\end{array}$ & $\begin{array}{c}89870 \\
(100.0 \%)\end{array}$ \\
\hline
\end{tabular}

a Students who reported don't know were excluded from analysis 
Cross-Tabs for Free/Reduced Lunch and Question 19 Responses

\begin{tabular}{lcccccc}
\hline \multicolumn{2}{l}{ Eligible for free or reduced-price lunch } & 0 days & 1 day & 2 days & 3 or more days & Total \\
\hline \multirow{2}{*}{ Yes } & $n$ & 19761 & 9552 & 6969 & 6525 & 42807 \\
& $(\%)$ & $(46.2 \%)$ & $(22.3 \%)$ & $(16.3 \%)$ & $(15.2 \%)$ & $(100.0 \%)$ \\
\multirow{2}{*}{ No } & $n$ & 24718 & 11944 & 7287 & 5990 & 49939 \\
& $(\%)$ & $(49.5 \%)$ & $(23.9 \%)$ & $(14.6 \%)$ & $(12.0 \%)$ & $(100.0 \%)$ \\
\multirow{2}{*}{ Total } & $n$ & 44479 & 21496 & 14256 & 12515 & 92746 \\
& $(\%)$ & $(48.0 \%)$ & $(23.2 \%)$ & $(15.4 \%)$ & $(13.5 \%)$ & $(100.0 \%)$ \\
\hline
\end{tabular}

Cross-Tabs for Free/Reduced Lunch and Question 21 Responses

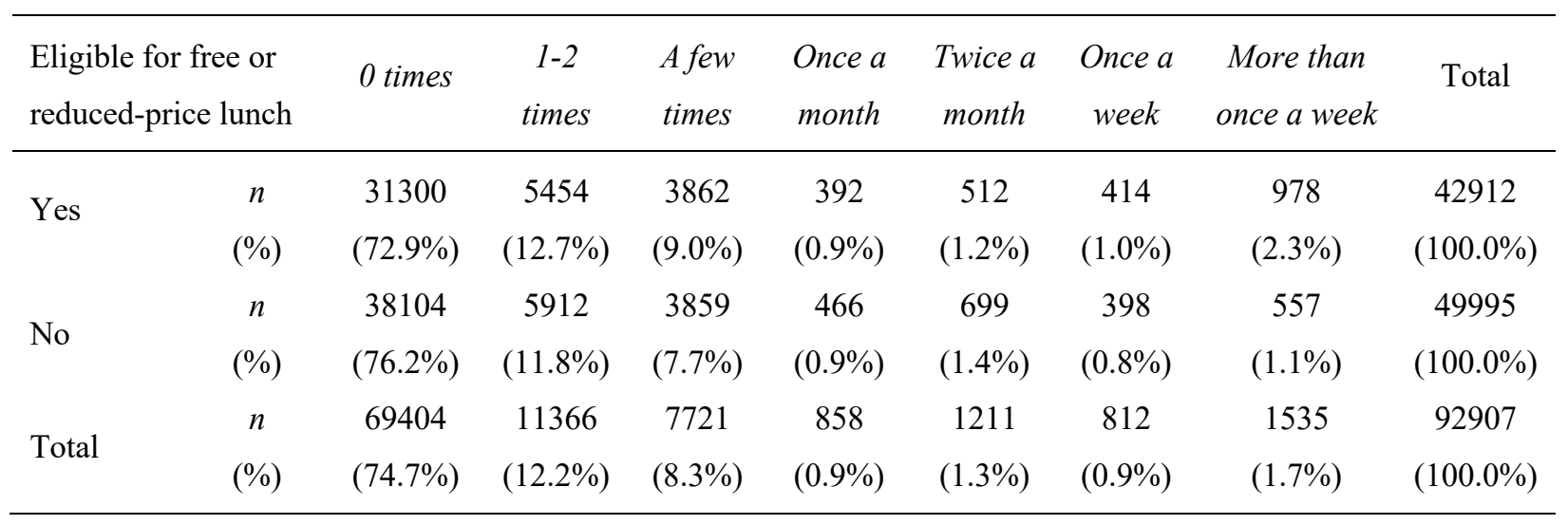

